Berichte aus dem

Institut für Systemdynamik

Universität Stuttgart

Knut Graichen

\title{
Feedforward Control Design for Finite-Time Transition Problems of Nonlinear Systems with Input and Output Constraints
}

D 93 (Diss. Universität Stuttgart)

Shaker Verlag

Aachen 2006 
Bibliographic information published by the Deutsche Nationalbibliothek The Deutsche Nationalbibliothek lists this publication in the Deutsche Nationalbibliografie; detailed bibliographic data are available in the Internet at http://dnb.d-nb.de.

Zugl.: Stuttgart, Univ., Diss., 2006

Copyright Shaker Verlag 2006

All rights reserved. No part of this publication may be reproduced, stored in a retrieval system, or transmitted, in any form or by any means, electronic, mechanical, photocopying, recording or otherwise, without the prior permission of the publishers.

Printed in Germany.

ISBN-10:3-8322-5747-0

ISBN-13: 978-3-8322-5747-7

ISSN 1863-9046

Shaker Verlag GmbH • P.O. BOX 101818 - D-52018 Aachen

Phone: 0049/2407/9596-0 - Telefax: 0049/2407/9596-9

Internet:www.shaker.de•e-mail:info@shaker.de 


\title{
Feedforward Control Design for Finite-Time Transition Problems of Nonlinear Systems with Input and Output Constraints
}

\author{
Von der Fakultät Maschinenbau der Universität Stuttgart \\ zur Erlangung der Würde eines \\ Doktor-Ingenieurs (Dr.-Ing.) genehmigte Abhandlung
}

\author{
Vorgelegt von \\ Knut Graichen \\ geboren in Dresden
}

Hauptberichter: $\quad$ Prof. Dr.-Ing. Dr.h.c. M. Zeitz

Mitberichter: Univ.-Prof. Dr.techn. A. Kugi

Tag der mündlichen Prüfung: 7. November 2006

Institut für Systemdynamik der Universität Stuttgart

2006 



\section{Vorwort}

Die vorliegende Arbeit entstand in den Jahren 2003 bis 2006 während meiner Tätigkeit als wissenschaftlicher Mitarbeiter am Institut für Systemdynamik (ehemals Institut für Systemdynamik und Regelungstechnik) der Universität Stuttgart.

Mein besonderer Dank gilt Herrn Prof. Dr.--Ing. Dr.h.c. M. Zeitz für die ausgezeichnete und engagierte Betreuung dieser Arbeit. Die zahlreichen Diskussionen und wertvollen Anregungen sowie die eingeräumten fachlichen Freiräume und Gestaltungsmöglichkeiten haben wesentlich zum Gelingen dieser Arbeit beigetragen. Ebenfalls herzlich danken möchte ich Herrn Univ.-Prof. Dr.techn. A. Kugi für sein lebhaftes Interesse an den Ergebnissen der Arbeit und die freundliche Übernahme des Mitberichts.

Außerdem bedanke ich mich bei dem Institutsleiter, Herrn Prof. Dr.-Ing. O. Sawodny, sowie bei Herrn Prof. Dr.-Ing. Dr.h.c.mult E.D. Gilles, der das Institut bis 2005 geleitet hat, für die günstigen Arbeitsbedingungen an diesem Institut. Den Kolleginnen und Kollegen am Institut danke ich für das sehr gute Arbeitsklima und die stete Hilfsbereitschaft.

Besonders danke ich Veit Hagenmeyer für die vielen fruchtbaren Diskussionen und die harmonische Zusammenarbeit auch während seiner Tätigkeit bei BASF Aktiengesellschaft. Nicht minder danken möchte ich Thomas Meurer, Matthias Bitzer und Tilman Utz für das sehr gute Zusammenspiel in der Gruppe von Herrn Prof. Zeitz. Tilman Utz war sowohl im Rahmen seiner studentischen Hilfstätigkeiten als auch als zeitlich befristeter Mitarbeiter bei Herrn Prof. Zeitz eine große Hilfe und Unterstützung.

Ebenfalls danken möchte ich Christian Bermes, Michael Treuer und Peter Wieland, die im Rahmen ihrer Studien- und Diplomarbeiten einen wertvollen Beitrag geleistet haben. Insbesondere die umfangreiche Unterstützung von Michael Treuer bei den Experimenten mit dem Doppel- und Dreifachpendel war eine wertvolle Hilfe. Schließlich, aber nicht zuletzt, danke ich Thomas Kiefer für die gute Zusammenarbeit bei dem "Helikopter-Projekt" in Saarbrücken sowie Rolf Findeisen für zahlreiche Diskussionen und Kommentare zu dieser Arbeit. 
"Would you tell me, please, which way I ought to go from here?"

"That depends a good deal on where you want to get to", said the Cat.

Lewis Carroll

Alice in Wonderland 


\section{Contents}

List of symbols $\quad$ VIII

Abstract

German summary / Deutsche Kurzfassung XIII

1 Introduction $\quad 1$

1.1 Feedforward control tasks . . . . . . . . . . . . . . . . . . . . . 2

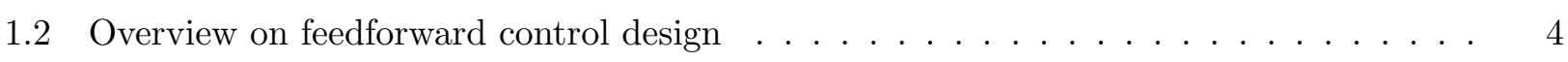

1.3 Goals and outline of the thesis $\ldots \ldots \ldots \ldots \ldots$

2 Feedforward control design for nonlinear SISO systems $\quad 9$

2.1 Finite-time transition between stationary setpoints . . . . . . . . . . . . . 9

2.1.1 Transition problem in flat coordinates . . . . . . . . . . . . . . 10

2.1.2 Transition problem in input-output coordinates . . . . . . . . . . . . . . . 12

2.2 Inversion-based feedforward control design . . . . . . . . . . . . . . . . . 14

2.2 .1 Boundary value problem of the internal dynamics . . . . . . . . . . . . . . 15

2.2 .2 Output trajectory with free parameters . . . . . . . . . . . . . 16

2.3 Numerical solution of the BVP with free parameters . . . . . . . . . . . . 19

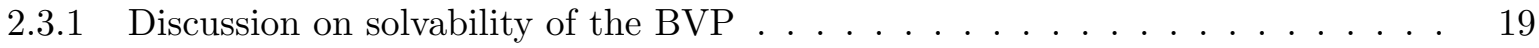

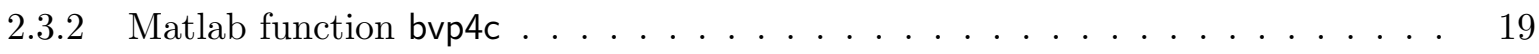

2.4 Example - Swing-up of the double pendulum on a cart $\ldots \ldots \ldots$. . . . . . . 21

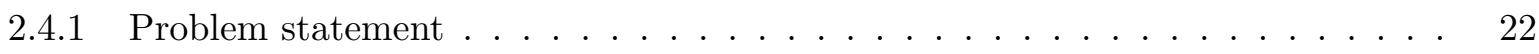

2.4.1.1 Equations of motion . . . . . . . . . . . . . . 22

2.4.1.2 Swing-up problem . . . . . . . . . . . . . . . . 24

2.4 .2 Feedforward control design . . . . . . . . . . . . . . . . . 24

2.4.2.1 Boundary value problem of the internal dynamics . . . . . . . . 25

2.4.2.2 Numerical results . . . . . . . . . . . . . . . . . 25

2.4 .3 Experimental validation . . . . . . . . . . . . . . . . 27

2.4.3.1 Experimental setup of the double pendulum . . . . . . . . . . . 27

2.4.3.2 Optimization-based adjustment of model parameters . . . . . . . . 28

2.4.3.3 Feedback control design . . . . . . . . . . . . . . . . . . . . . . . . . . . . . . 30

2.4.3.4 Experimental results . . . . . . . . . . . . . . . . . 32

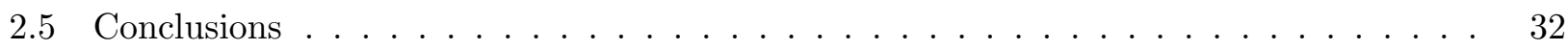


3 Feedforward control design for nonlinear SISO systems with input constraints 34

3.1 Boundary value problems for constrained input . . . . . . . . . . . . 35

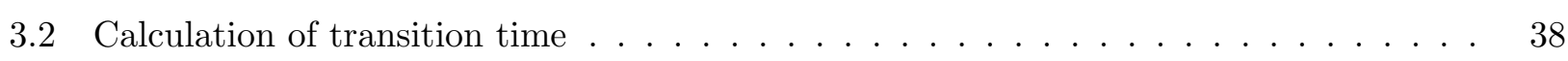

3.2.1 Aggressiveness measure for the feedforward control . . . . . . . . . . . 38

3.2 .2 Boundary value problem for feedforward aggressiveness . . . . . . . . . . 40

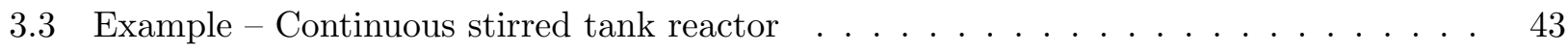

3.3 .1 Reactor model . . . . . . . . . . . . . . . . . . . 43

3.3 .2 Input-output normal form . . . . . . . . . . . . . . . . 45

3.3.3 Feedforward control design for setpoint change . . . . . . . . . . . . . 47

3.3 .4 Numerical results . . . . . . . . . . . . . . . . . . . . . . 47

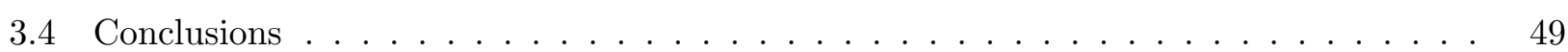

4 Feedforward control design for nonlinear SISO systems with output constraints 50

4.1 System representation for constrained output . . . . . . . . . . . . . 51

4.1.1 Successive incorporation of output constraints . . . . . . . . . . . . 51

4.1 .2 Choice of saturation functions . . . . . . . . . . . . . . . 53

4.1 .3 Calculation of saturation limits . . . . . . . . . . . . . . 54

4.2 Boundary value problem for constrained output . . . . . . . . . . . . 56

4.2 .1 Calculation of boundary conditions . . . . . . . . . . . . . . 56

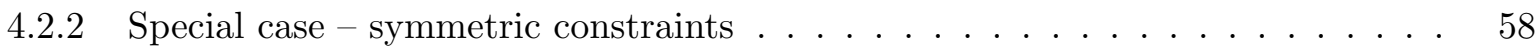

4.2 .3 Setup function with free parameters $\ldots \ldots \ldots \ldots \ldots$

4.3 Incorporation of input constraints . . . . . . . . . . . . . . . . 60

4.4 Example - Side-stepping of the triple inverted pendulum on a cart . . . . . . . . . 63

4.4 Problem statement . . . . . . . . . . . . . . . . . 64

4.4.1.1 Equations of motion . . . . . . . . . . . . . . 64

4.4.1.2 Side-stepping problem . . . . . . . . . . . . . . 65

4.4 .2 Feedforward control design $\ldots \ldots \ldots \ldots \ldots$

4.4.2.1 Boundary value problems for constrained output . . . . . . . . . 67

$4.4 .2 .2 \quad$ Numerical results . . . . . . . . . . . . . . . . . 68

4.4 .3 Experimental validation $\ldots \ldots \ldots \ldots \ldots \ldots$

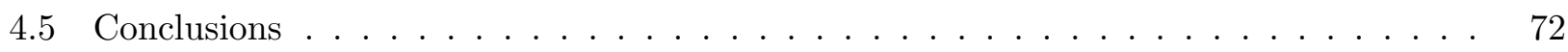

$\begin{array}{lll}5 & \text { Feedforward control design for nonlinear MIMO systems } & 73\end{array}$

5.1 Finite-time transition between stationary setpoints . . . . . . . . . . . . 73

5.1 .1 Input-output normal form . . . . . . . . . . . . . . . . . 74

5.1 .2 Inversion-based feedforward control design . . . . . . . . . . . 76

5.2 Incorporation of input constraints . . . . . . . . . . . . . . . . . 79 
5.3 Incorporation of output constraints . . . . . . . . . . . . . . . 84

5.3 .1 System representation for constrained outputs . . . . . . . . . . . . 84

5.3.2 Boundary value problems for constrained outputs . . . . . . . . . . . . . . 85

5.4 Incorporation of input and output constraints . . . . . . . . . . . . . . . 87

5.5 Example - Flight maneuver of the 3 DOF helicopter $\ldots \ldots \ldots$. . . . . . . . 91

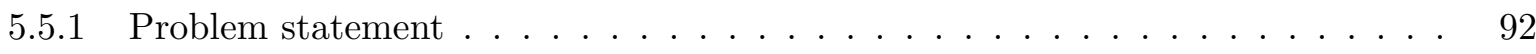

5.5.1.1 Helicopter model . . . . . . . . . . . . . . . . . . 92

5.5.1.2 Finite-time transition problem . . . . . . . . . . . . . . 93

5.5.2 Transition problem in input-output coordinates . . . . . . . . . . . . . 93

5.5 .3 Feedforward control design . . . . . . . . . . . . . . . . 94

5.5 .4 Incorporation of input constraints . . . . . . . . . . . . . . . . . . . . . . . . . .

5.5 .5 Incorporation of output constraints . . . . . . . . . . . . . 98

5.5.5.1 System representation for constrained output . . . . . . . . . . . 98

5.5.5.2 Boundary value problem for constrained output . . . . . . . . . . 100

5.5.5.3 Numerical results . . . . . . . . . . . . . . . . . . . 101

5.5 .6 Feedback controller . . . . . . . . . . . . . . . . . . 102

5.5 .7 Experimental results . . . . . . . . . . . . . . . . . . 102

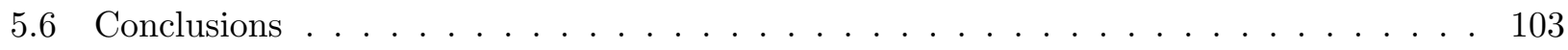

6 Conclusions and outlook $\quad 105$

\section{Appendix}

A Setup functions for output trajectory with free parameters $\quad 108$

A.1 Polynomial series . . . . . . . . . . . . . . . . . . . . . 108

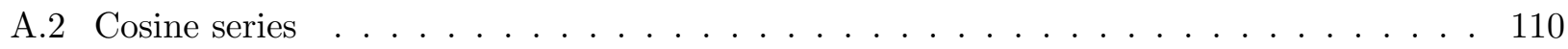

B Numerical solution of two-point boundary value problems $\quad 111$

B.1 Collocation method . . . . . . . . . . . . . . . . . . 111

B.2 Finite-difference method . . . . . . . . . . . . . . . . . . . . . 113

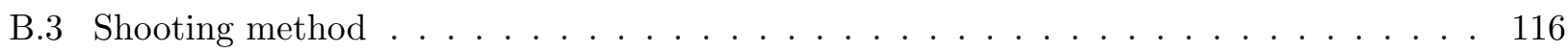

C Incorporation of output constraints with non-asymptotic saturation functions 118

C.1 Calculation of non-asymptotic saturation limits . . . . . . . . . . . . . . . . 118

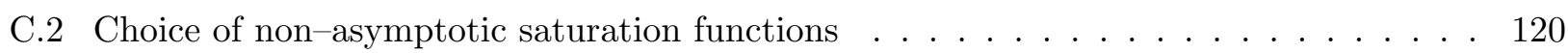

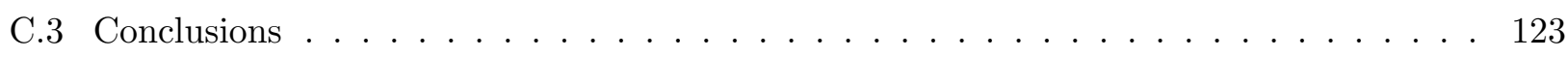




\section{List of symbols}

The following list only contains symbols that are used continuously throughout the text. Local symbols are not listed.

\section{Variables}

$\boldsymbol{p} \quad$ set of free parameters

$t \quad$ time

$u \quad$ scalar input

$u^{*} \quad$ scalar feedforward control

$u_{\Phi}^{*} \quad$ scalar feedforward control for $y^{*(r)}=\Phi(t, \boldsymbol{p})$

$u_{v}^{*} \quad$ scalar feedforward control for $y_{v}^{*(r)}$ in (4.32)

$u_{0}^{*}, u_{T}^{*} \quad$ scalar stationary inputs

$\Delta u \quad$ feedback correction: $\Delta u=\boldsymbol{k}^{\top}(t)\left(\boldsymbol{x}^{*}-\boldsymbol{x}\right)$ with feedback gains $\boldsymbol{k}(t)$

$\boldsymbol{u} \quad m$-vector of inputs

$\boldsymbol{u}^{*} \quad m$-vector of feedforward controls

$\boldsymbol{u}_{\Phi}^{*} \quad m$-vector of feedforward controls for $y_{k}^{*\left(r_{k}\right)}=\Phi_{k}\left(t, \boldsymbol{p}_{k}\right), k=1, \ldots, m$

$\boldsymbol{u}_{v}^{*} \quad m$-vector of feedforward controls for $y_{v, k}^{*\left(r_{k}\right)}$ in (5.51)

$\boldsymbol{u}_{0}^{*}, \boldsymbol{u}_{T}^{*} \quad m$-vector of stationary inputs

$v \quad$ new input for saturation function $\psi_{r+1}\left(v, \psi_{r+1}\left(\boldsymbol{\xi}_{r}\right)\right)$

$\boldsymbol{x} \quad n$-vector of states

$\boldsymbol{x}^{*} \quad n$-vector of desired states

$\boldsymbol{x}_{0}^{*}, \boldsymbol{x}_{T}^{*} \quad n$-vector of stationary states

$\Delta \boldsymbol{x} \quad$ tracking error: $\Delta \boldsymbol{x}=\boldsymbol{x}-\boldsymbol{x}^{*}$

y scalar output

$y^{*} \quad$ scalar desired output 
$y_{0}^{*}, y_{T}^{*} \quad$ scalar stationary outputs

$\boldsymbol{y}_{k} \quad r_{k}$-vector of derivatives $\boldsymbol{y}_{k}=\left[y_{k}, \dot{y}_{k}, \ldots, y_{k}^{\left(r_{k}-1\right)}\right]^{\boldsymbol{\top}}$ of the $k$-th output $y_{k}$

z scalar flat output

$z^{*} \quad$ scalar desired flat output

$z_{0}^{*}, z_{T}^{*} \quad$ scalar stationary flat outputs

$\boldsymbol{\eta} \quad(n-r)$-vector of internal dynamics states

$\boldsymbol{\eta}^{*} \quad(n-r)$ - vector of desired internal dynamics states

$\boldsymbol{\eta}_{0}^{*}, \boldsymbol{\eta}_{T}^{*} \quad(n-r)$ - vector of stationary internal dynamics states

$\xi_{i} \quad i$-th coordinate of saturation function $\psi_{i}\left(\xi_{i}, \psi_{i}^{ \pm}\left(\boldsymbol{\xi}_{i-1}\right)\right), i=1, \ldots, r$

$\xi_{i, 0}, \xi_{i, T} \quad$ stationary states for $\xi_{i}$

$\boldsymbol{\xi}_{i} \quad i$-vector of coordinates: $\boldsymbol{\xi}_{0}=\emptyset, \boldsymbol{\xi}_{i}=\left[\xi_{1}, \ldots, \xi_{i}\right]^{\top}, i=1, \ldots, r$

$\boldsymbol{\xi}_{i, 0}, \boldsymbol{\xi}_{i, T} \quad i$-vector of stationary states for $\boldsymbol{\xi}_{i}$

\section{Functions}

$\boldsymbol{f} \quad$ vector field of system (2.1) and (5.1)

$h \quad$ output function $y=h(\boldsymbol{x})$

$\alpha \quad$ function of input-output dynamics

$\alpha^{-}, \alpha^{+} \quad$ constraints for $y^{*(r)} \in\left[\alpha^{-}, \alpha^{+}\right]$stemming from the input constraints $u^{*} \in\left[u^{-}, u^{+}\right]$

$\hat{\alpha} \quad$ setup function $y^{*(r)}=\hat{\alpha}$ to incorporate the input constraints $u^{*} \in\left[u^{-}, u^{+}\right]$

$\boldsymbol{\alpha} \quad$ vector function of input-output dynamics: $\boldsymbol{\alpha}=\left[\alpha_{1}, \ldots, \alpha_{m}\right]^{\top}$

$\boldsymbol{\beta} \quad$ vector field of internal dynamics of dimension $n-r$

$\overline{\boldsymbol{\beta}} \quad$ vector field of internal dynamics with output variables serving as input ("tracking dynamics")

$\phi \quad$ nonlinear coordinate transformation (diffeomorphism)

$\Phi \quad$ setup function for $\hat{\alpha}=\Phi(t, \boldsymbol{p})$

$\psi_{i} \quad i$-th saturation function $\psi_{i}\left(\cdot, \psi_{i}^{ \pm}\left(\boldsymbol{\xi}_{i-1}\right)\right), i=1, \ldots, r+1$

$\psi_{i}^{-}, \psi_{i}^{+} \quad i$-th saturation limits $\left.\psi_{i}^{ \pm}\left(\boldsymbol{\xi}_{i-1}\right)\right), i=1, \ldots, r+1$

$\boldsymbol{\psi}_{i} \quad$ vector of saturation functions: $\boldsymbol{\psi}_{2}=\psi_{2}, \quad \boldsymbol{\psi}_{i}=\left[\psi_{2}, \ldots, \psi_{i}\right]^{\top}, i=3, \ldots, r+1$

$\widehat{\psi}_{r+1} \quad$ setup function $\dot{\xi}_{r}=\widehat{\psi}_{r+1}\left(\boldsymbol{\xi}_{r}, \boldsymbol{\eta}^{*}, v\right)$ to incorporate the input constraints $u^{*} \in\left[u^{-}, u^{+}\right]$

$\Upsilon \quad$ setup function for $y^{*}(t)=\Upsilon(t, \boldsymbol{p})$ 


\section{Scalars}

$i, j, k \quad$ indices

$m \quad$ dimension of input vector $\boldsymbol{u}$ and outputs $y_{k}, k=1, \ldots, m$

$n \quad$ dimension of state vector $\boldsymbol{x}$

$q_{k} \quad$ distribution of free parameters $\boldsymbol{p}_{k}=\left(p_{k, 1}, \ldots, p_{k, q_{k}}\right)$ to outputs $y_{k}, k=1, \ldots, m$

$r \quad$ (vector) relative degree

$T \quad$ transition time relative degree

$u^{-}, u^{+} \quad$ input constraints $u^{*} \in\left[u^{-}, u^{+}\right]$

$y_{i}^{-}, y_{i}^{+} \quad$ output constraints $y^{*(i)} \in\left[y_{i}^{-}, y_{i}^{+}\right], i=0,1, \ldots, r$

$\delta \quad$ aggressiveness measure for feedforward control $u^{*}(t)$

$\rho \quad$ differentiability index of output trajectory $y^{*}(t) \in \mathcal{C}^{\rho}$

\section{Abbreviations}

$(\cdot)^{ \pm} \quad$ compact notation for constraints $(\cdot)^{-}$and $(\cdot)^{+}$

BC boundary condition

BVP boundary value problem

CSTR continuous stirred tank reactor

DOF degree-of-freedom

FB feedback control

FF feedforward control

IVP initial value problem

MIMO multiple-input multiple-output

ODE ordinary differential equation

SISO single-input single-output 


\section{Abstract}

Feedforward controls are used in many practical control applications as an extension of the feedback control loop to separately design the tracking performance by the feedforward part and the robustness and closed-loop stability by the feedback part ("two-degree-of-freedom control"). However, compared to the broad spectrum of methods for designing the feedback control, only few systematic approaches are available for feedforward control design. This methodological gap is mainly caused by the required inversion of the input-output behavior and the respective difficulties arising with nonlinear systems. Especially if the considered system is nonminimum-phase, the stable numerical integration of the unstable internal dynamics is nontrivial and leads to a noncausal feedforward control. Moreover, the feedforward design is further complicated if constraints on the input or the output trajectories have to be satisfied, e.g. to account for actuator constraints or to limit the typical counter-swing behavior of nonminimum-phase systems.

A particularly convenient feedforward design task is the transition between two stationary setpoints in a finite time interval. Practical applications are e.g. rest-to-rest motions in mechatronics or load changes in process control. For these transition problems where in particular the feedforward trajectories can be calculated offline, the difficulties concerning the instability of the internal dynamics and the corresponding noncausality of the feedforward control can be avoided.

In this thesis, the finite-time transition problem between stationary setpoints is treated as a two-point boundary value problem (BVP) in the coordinates of the input-output normal form. In order to solve the overdetermined BVP of the internal dynamics and to ensure the causality of the feedforward control, a sufficient number of free parameters is provided in the setup function of the output trajectory. The resulting BVP with free parameters can be solved with standard numerical methods, e.g. with the MATLAB function bvp4c. Thereby, the free parameters determine the shape of the output trajectory. For instance, in the case of nonminimum-phase systems, the output may contain an initial undershoot. The feedforward design approach is illustrated for the swing-up of the double pendulum on a cart. The swing-up is experimentally validated by calculating time-varying gains for a linear state feedback control which stabilizes the pendulum along the nominal feedforward trajectories.

The above-mentioned input constraints can be incorporated in the feedforward control design by augmenting the internal dynamics BVP by a corresponding BVP for the output. Thereby, 
the feedforward control is guaranteed to stay inside the constraints by case-dependently planning the highest time derivative of the output. Moreover, the transition time of the finite-time setpoint transition can be determined as part of the BVP solution by rating the aggressiveness of the feedforward control with respect to the input constraints. This concept is applied to a setpoint transition scenario of a continuous stirred tank reactor (CSTR) with constraints on the cooling power.

In order to account for constraints on the output and its time derivatives, the previous output BVP is replaced by a suitable dynamic system (and corresponding boundary conditions), which automatically constrains the output due to its particular construction. Moreover, the input constraints can be treated as constraints on the highest time derivative of the output. In this way, the input and output constraints are systematically incorporated in the BVP formulation of the finite-time transition problem, which again can be solved e.g. with the MATLAB function bvp4c. The approach is illustrated for the side-stepping maneuver of the triple inverted pendulum on a cart with constraints on the cart position, velocity, and acceleration. The experimental results of the side-stepping maneuver shows the accuracy of the feedforward control in combination with a stabilizing state feedback controller.

Moreover, the feedforward control design can be extended to nonlinear multiple-input multipleoutput (MIMO) systems in a straightforward manner. Similar to the single-input single-output (SISO) case, the finite-time transition problem is treated as a two-point BVP in the coordinates of the MIMO input-output normal form. Thereby, some freedom exists how the free parameters are distributed to the multiple outputs. The constraints on each input and output channel are incorporated by adapting the results of the SISO case. The MIMO design is applied to a flight maneuver of a 3DOF helicopter with constraints on both inputs and the pitch angle. The flight maneuver is experimentally validated using a linear state feedback control with time-varying gains in addition to the feedforward control.

Particularly the swing-up and side-stepping of the double/triple pendulums with highly nonlinear and unstable dynamics illustrate that the presented offline approach for feedforward control design is applicable to nonlinear, nonminimum-phase, and unstable systems. This is due to the fact that algebraic BVP solution techniques like collocation (implemented in bvp4c) are not based on numerical integration and thus handle the BVPs regardless of their stability properties. Moreover, the BVP formulation of the feedforward design ensures that the initial and terminal stationary setpoints are exactly met. Hence, the setpoint transition is performed in finite-time and the causality of the feedforward control is guaranteed.

The feedforward control design is also applicable to differentially flat systems, where all system variables can be expressed in terms of a flat output and its time derivatives. The feedforward design is nevertheless based on a BVP solution if constraints on the input or the flat output shall be considered for the finite-time transition problem. A possible extension of the feedforward control design under input and output constraints concerns e.g. the minimization of a cost functional with respect to additional free parameters provided in the output trajectory. 


\section{Deutsche Kurzfassung}

\section{Vorsteuerungsentwurf für Arbeits- punktwechsel nichtlinearer Systeme mit Ein- und Ausgangsbeschränkungen}

In vielen regelungstechnischen Anwendungen werden Vorsteuerungen als Erweiterung des eigentlichen Regelkreises eingesetzt, um das Führungs- und Störverhalten einer Folgeregelung getrennt voneinander zu entwerfen ("Zwei-Freiheitsgrade-Regelung"). Allerdings gibt es im Unterschied zu der Vielzahl von Methoden für den Reglerentwurf nur wenige Verfahren zum systematischen Entwurf einer Vorsteuerung, was hauptsächlich auf die damit verbundene Systeminversion zurückzuführen ist. Vorallem im Falle von nichtlinearen nichtminimalphasigen Systemen ist die stabile numerische Integration der instabilen internen Dynamik denkbar schwierig und führt zu einer nichtkausalen Vorsteuerung. Der Vorsteuerungsentwurf wird zusätzlich erschwert, falls Eingangs- und/oder Ausgangsbeschränkungen berücksichtigt werden sollen.

Eine häufig auftretende Steuerungsaufgabe ist der Arbeitspunktwechsel. Typische Beispiele dafür sind Positionswechsel in der Robotik oder Anfahrvorgänge und Lastwechsel bei verfahrenstechnischen Prozessen. Für diese Anwendungen, bei denen zusätzlich die nominellen Trajektorien offline berechnet werden können, kann die Problematik der instabilen internen Dynamik und die damit verbundene Nichtkausalität der Steuerung umgangen werden.

In dieser Arbeit wird der betrachtete Arbeitspunktwechsel als eine Zwei-Punkt-Randwertaufgabe (RWA) in den Koordinaten der Ein-/Ausgangs-Normalform definiert. Um die überbestimmte RWA der internen Dynamik lösen zu können und die Kausalität der Steuerung zu gewährleisten, werden freie Parameter in der Planung der Ausgangstrajektorie zur Verfügung gestellt. Die RWA mit freien Parametern kann mit numerischen Standardverfahren, wie z.B. der MatlaB-Funktion bvp4c gelöst werden. Dabei wird die Form der Ausgangstrajektorie maßgeblich durch die freien Parameter bestimmt. Im Falle von nichtminimalphasigen Systemen kann der Ausgang z.B. ein Gegenschwingen aufweisen. Als Beispiel für den Vorsteuerungsentwurf wird das Aufschwingen eines Doppelpendels auf einem Wagen betrachtet und experimentell validiert. Dazu wird ein linearer Zustandsregler mit zeitvarianten Verstärkungen entworfen, um das Doppelpendel entlang der nominellen Trajektorien zu stabilisieren. 
Die bereits erwähnten Stellgrößenbeschränkungen können in dem Entwurfsverfahren berücksichtigt werden, indem die RWA der internen Dynamik um eine entsprechende RWA für den Ausgang erweitert wird. Dabei wird die höchste Ausgangsableitung fallabhängig angesetzt, um das Einhalten der Eingangsbeschränkungen zu garantieren. Des Weiteren lässt sich in Abhängigkeit einer gewünschten "Aggressivität" der Vorsteuerung die Transitionszeit des Arbeitspunktwechsels zusammen mit der RWA berechnen. Als Anwendungsbeispiel dient das Modell eines kontinuierlich betriebenen Rührkesselreaktors (CSTR) mit begrenzter Kühlleistung.

Um Beschränkungen des Ausgangs und seiner Zeitableitungen im Vorsteuerungsentwurf einzuarbeiten, wird die RWA für den Ausgangs durch ein geeignet konstruiertes System (und die entsprechenden Randbedingungen) ersetzt, das die Ausgangsbeschränkungen aufgrund seiner speziellen Struktur direkt erfüllt. Darüber hinaus lassen sich Eingangsbeschränkungen als Beschränkungen der höchsten Ausgangsableitung auffassen und somit in der neuen RWA berücksichtigen. Auf diese Weise werden die Ein- und Ausgangsbeschränkungen direkt in der Formulierung der RWA eingearbeitet, deren Lösung mit der bereits erwähnten MATLAB-Funktion bvp4c erfolgt. Als Beispiel wird das seitliche Versetzen des invertieren Dreifachpendels mit beschränkter Wagenposition, -geschwindigkeit und -beschleunigung betrachtet. Die experimentellen Ergebnisse zeigen eine hohe Genauigkeit und Reproduzierbarkeit der Vorsteuerung in Verbindung mit einem stabilisierenden Zustandsregler.

Der Vorsteuerungsentwurf kann direkt auf nichtlineare Mehrgrößensysteme übertragen werden. Dazu wird wie im Eingrößenfall der Arbeitspunktwechsel als Zwei-Punkt-RWA in den Koordinaten der entsprechenden Ein-/Ausgangs-Normalform definiert. Im Mehrgrößenfall treten dabei zusätzliche Freiheitsgrade auf, wie die freien Parameter für die Lösbarkeit der RWA auf die einzelnen Ausgänge verteilt werden. Der Mehrgrößenentwurf wird für ein Flugmanöver (360-Drehung) eines 3-Freiheitsgrade-Helikopters mit Beschränkungen der beiden Eingänge und des Neigewinkels veranschaulicht. Für die experimentelle Realisierung wird zusätzlich zur Vorsteuerung ein linearer Zustandsregler mit zeitvarianten Verstärkungen verwendet.

Das vorgestellte Offline-Verfahren zum Entwurf von Vorsteuerungen ist gleichermaßen anwendbar auf (nicht)lineare und (nicht)minimalphasige Systeme, da die algebraische Lösung der RWA z.B. mit dem Kollokationsverfahren (bvp4c) keine numerische Integration verwendet und somit auch für instabile Differentialgleichungen eingesetzt werden kann. Dies ist insbesondere für die Steuerung des Doppel- und Dreifachpendels aufgrund der komplexen und instabilen internen Dynamik von Bedeutung. Darüber hinaus gewährleisten die stationären Randbedingungen der RWA, dass der Arbeitspunktwechsel in dem definierten Zeitintervall realisiert wird und somit die Nichtkausalität der Vorsteuerung bei nichtminimalphasigen Systemen vermieden wird.

Der Vorsteuerungsentwurf lässt sich auch auf flache Systeme anwenden, bei denen die Steuerung rein algebraisch in Abhängigkeit des flachen Ausgangs und seiner Zeitableitungen berechnet werden kann. Dennoch ist in diesem Fall die Lösung einer RWA notwendig, wenn Beschränkungen des Eingangs oder des flachen Ausgangs berücksichtigt werden sollen. Eine mögliche Erweiterung des Vorsteuerungsentwurfes betrifft z.B. die Minimierung eines Kostenfunktionals anhand weiterer Parameter, die im Ansatz der Ausgangstrajektorie zur Verfügung gestellt werden. 


\section{Chapter 1}

\section{Introduction}

In many practical control applications where high tracking performance is required, the twodegree-of-freedom (2DOF) control scheme shown in Figure 1.1 is used as a straightforward extension of the classical feedback control (Horowitz, 1963; Reinschke, 2006). The feedback control $\Sigma_{F B}$ is designed such that the system $\Sigma$ is appropriately stabilized and robustified against model uncertainties and disturbances. The feedforward control $\Sigma_{F F}$ is applied as the second degree-of-freedom to achieve the desired tracking performance of the output $y$. The signal generator $\Sigma^{*}$ provides the reference trajectory $y^{*}(t)$ for both the feedback and the feedforward control.

The feedforward control $\Sigma_{F F}$ has to provide a nominal input trajectory $u^{*}(t)$ which forces the system $\Sigma$ to follow the desired output trajectory $y^{*}(t)$ in the nominal case. This in particular illustrates the importance of appropriate motion planning, i.e. the design of attainable and realizable output trajectories $y^{*}(t)$ provided by the signal generator $\Sigma^{*}$. Moreover, an essential requirement for the feedforward control design is the knowledge of a suitable and sufficiently accurate system model $\Sigma$, since the calculation of the input trajectory $u^{*}(t)$ is based on the inverse system representation $\Sigma^{-1}$. In any case, the inaccuracy of the feedforward control $\Sigma_{F F}=\Sigma^{-1}$ has to be compensated by the feedback control $\Sigma_{F B}$.

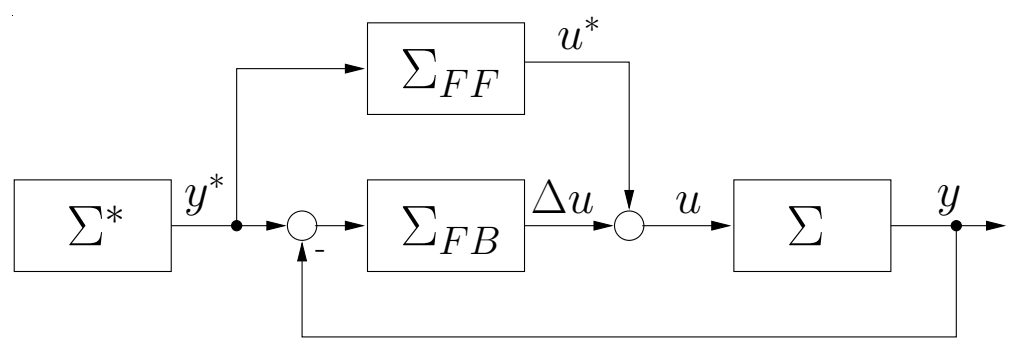

Figure 1.1: Two-degree-of-freedom (2DOF) control scheme (Horowitz, 1963) with system $\Sigma$, feedback control $\Sigma_{F B}$, feedforward control $\Sigma_{F F}$, and signal generator $\Sigma^{*}$. 


\section{$1.1 \quad$ Feedforward control tasks}

Roughly speaking, there are two types of application for feedforward control design. A typical feedforward design problem is the finite-time transition between two stationary setpoints. Practical applications are e.g. rest-to-rest motion in mechatronical systems or heat-up, change of operating points, and shutdown of a reactor in process control. A more specific feedforward design problem is output tracking, i.e. a desired output trajectory $y^{*}(t)$ is given and a feedforward control $u^{*}(t)$ has to be calculated such that the system follows $y^{*}(t)$.

An important issue is if the respective application requires an offline or online design of the feedforward control. The offline design treats the actual design and implementation separately by shifting the numerical solution of the feedforward design to the precalculation and storing the nominal trajectories in look-up tables. This is particularly convenient in the presence of constraints, e.g. on the input and states of the system, which significantly increases the numerical load. On the other hand, the online feedforward design requires the simultaneous design and implementation in real-time, which naturally restricts the complexity of the underlying calculations. However, the online design provides a higher flexibility, if the feedforward control has to adapt to changing model parameters or the application requires the transition between any (and possibly beforehand unknown) operating point. In these cases, the offline calculation would lead to complex and multi-dimensional look-up tables. Table 1.1 gives a rough overview

\begin{tabular}{|c|c|c|}
\hline & Finite-time setpoint transition & Output tracking \\
\hline $\begin{array}{l}\text { Offline feed- } \\
\text { forward design } \\
\text { (look-up table) }\end{array}$ & $\begin{array}{l}\text { Typical applications: } \\
\text { - limited number of (beforehand known) } \\
\text { operating points } \\
\text { - fast or repetitive transitions } \\
\text { - constraints or cost optimization } \\
\text { Examples: rest-to-rest motion for } \\
\text { - flexible manipulators } \\
\text { (Benosman et al., 2004) } \\
\text { - automotive gas-exchange valve } \\
\text { (Chung et al., 2006) }\end{array}$ & $\begin{array}{l}\text { Typical applications: } \\
\text { - desired output trajectory } y^{*}(t) \text { is } \\
\text { known beforehand } \\
\text { - fast, repetitive, or periodic trajectories } \\
\text { - constraints or cost optimization } \\
\text { Example: } \\
\text { - cutting or welding trajectories in } \\
\text { material processing }\end{array}$ \\
\hline $\begin{array}{l}\text { Online feed- } \\
\text { forward design }\end{array}$ & $\begin{array}{l}\text { Typical applications: } \\
\text { - wide range of (beforehand unknown) } \\
\text { operating points } \\
\text { - changing model parameters } \\
\text { Example: } \\
\text { - batch reactor with multiple products } \\
\text { (Petit et al., 2002; Hagenmeyer and } \\
\text { Nohr, 2005) }\end{array}$ & $\begin{array}{l}\text { Typical applications: } \\
\text { - instantaneous generation of desired } \\
\text { output trajectory } y^{*}(t) \\
\text { - changing model parameters } \\
\text { Examples: } \\
\text { - constrained online trajectory } \\
\text { generation (Zanasi et al., 2000) } \\
\text { - aircraft guidance (Miquel et al., 2005) } \\
\text { - } \text { anti-sway control of cranes } \\
\text { (Neupert et al., 2005) }\end{array}$ \\
\hline
\end{tabular}

Table 1.1: Overview on offline and online feedforward control design depending on its application. 
on typical applications and examples for finite-time transition problems and output tracking where an online or offline feedforward design is possible or required.

The focus of this thesis is on the offline feedforward control design for the finite-time transition between stationary setpoints. Compared to the more specific problem of output tracking where an output trajectory $y^{*}(t)$ is predefined, the finite-time transition between two stationary setpoints is a more general control task, since the output $y^{*}(t)$ is not restricted to a certain trajectory, but has to be determined in course of the feedforward control design. The appropriate planning of the output trajectory is of particular importance, if the feedforward control design is additionally complicated by input and output constraints on the feedforward control $u^{*}(t)$ and the desired output trajectory $y^{*}(t)$, as schematically shown in Figure 1.2. For instance, the input $u$ may be constrained by a limited actuator torque or limited cooling/heating power for chemical reactors. Constraints on the output $y$ and its time derivatives $y^{(i)}, i \geq 1$ are e.g. security constraints if $y$ represents a reactor's temperature or the position of a mechatronic device. $^{1}$

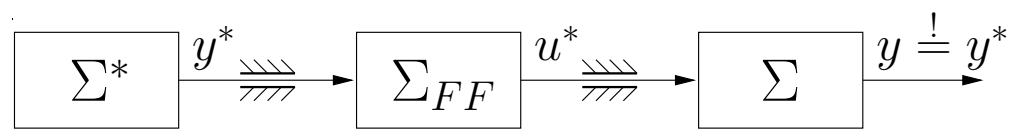

Figure 1.2: Open-loop control of system $\Sigma$ with signal generator $\Sigma^{*}$ and feedforward control $\Sigma_{F F}$ as well as schematically shown constraints on input $u^{*}$ and output $y^{*}$.

From a mathematical point of view, a finite-time transition forms a two-point boundary value problem with given initial and terminal stationary setpoints. Its solution by a feedforward control comprises the following design tasks:

- The signal generator $\Sigma^{*}$ must provide a desired output trajectory $y^{*}(t)$, which appropriately connects the initial and terminal stationary setpoints. The trajectory $y^{*}(t)$ is also used as reference for the output feedback control $\Sigma_{F B}$, see Figure 1.1.

- The feedforward control $\Sigma_{F F}$ provides the nominal input trajectory $u^{*}(t)$ which steers the system $\Sigma$ in open-loop mode from the initial to the terminal setpoint, such that its output $y$ follows the reference signal $y^{*}(t)$ in the nominal case, see Figure 1.2.

- If input and output constraints are present, the feedforward trajectory $u^{*}(t)$ as well as the desired output trajectory $y^{*}(t)$ have to satisfy these constraints.

The incorporation of input and output constraints clearly illustrates that both aspects of motion planning $y^{*}(t)$ and feedforward control design $u^{*}(t)$ are tightly coupled. On the one hand, the

\footnotetext{
${ }^{1}$ Although the consideration of input and output constraints is a special case of the more general problem of input and state constraints, they are considered here since the relation between input $u$ and output $y$ forms the basis for the feedforward control design, see Figure 1.2. This point of view also allows to incorporate the output constraints directly within the feedforward control design as described in detail in Chapter 4.
} 
output trajectory $y^{*}(t)$ has to connect the boundary points and must be planned realizable with respect to the system dynamics. On the other hand, the input and output constraints affect the feedforward control $u^{*}(t)$ and the output trajectory $y^{*}(t)$ simultaneously.

\subsection{Overview on feedforward control design}

In comparison to the broad spectrum of available design methods for feedback control, only few methods for feedforward control design are known - in contrast to the respective demand for industrial applications. The reason for this methodological gap is related to the system inversion required in course of the feedforward control design and to the respective difficulties arising with nonlinear systems.

The problem of invertibility was initially studied for linear systems by Brockett and Mesarovic (1965). More complete treatments were given by Sain and Massey (1969), and Silverman (1969). In these contributions, conditions are investigated for the construction of the left-inverse system corresponding to the original system. The linear results were extended by Hirschorn (1979) to nonlinear systems. Singh (1981) provided further results to extend the inversion procedure to a larger class of nonlinear systems. In all these works, the nominal input trajectory $u^{*}(t)$ is obtained by solving the left-inverse system as an initial value problem for a given desired output trajectory $y^{*}(t)$. However, if the system is nonminimum-phase, the left-inverse system is unstable, thus leading to an unbounded input trajectory $u^{*}(t)$ when the left-inverse system is solved as an initial value problem. This problem of nonminimum-phase systems can be illustrated for a simple linear example.

Example 1.1. Consider a linear controllable SISO system with the transfer function

$$
\Sigma: \quad G(s)=\frac{Y(s)}{U(s)}=\frac{b_{0}-b_{1} s}{a_{0}+a_{1} s+s^{2}}, \quad a_{i}, b_{i}>0, \quad i=0,1 .
$$

For a given sufficiently smooth output trajectory $y^{*}(t)$, the corresponding feedforward control $u^{*}(t)$ has to satisfy the input-output relation

$$
b_{0} u^{*}-b_{1} \dot{u}^{*}=a_{0} y^{*}+a_{1} \dot{y}^{*}+\ddot{y}^{*},
$$

which represents a first-order ODE for $u^{*}(t)$ in dependence of $y^{*}(t)$. The nominal feedforward control $u^{*}(t)$ can be obtained by solving (1.2) as an initial value problem with initial condition $u^{*}(0)=u_{0}$. For the parameters $b_{0}, b_{1}>0$, the system is nonminimum-phase with the zero of the transfer function (1.1) lying in the right half plane. Hence, the ODE (1.2) for $u^{*}(t)$ is unstable and its integration in forward time $t \in[0, \infty)$ leads to an unbounded solution $u^{*}(t)$.

The difficulty with the feedforward control design for nonminimum-phase systems has been known for a long time. With the advance of nonlinear control theory, Devasia et al. (1996) and Chen and Paden (1996) introduced a new approach for stable system inversion. The feedforward 
design is based on the input-output normal form (Isidori, 1995) of the considered system. If the system is nonminimum-phase, the internal dynamics is split in stable and unstable subsystems which are solved numerically by an iterative forward- and reverse-time integration. However, in the case of nonminimum-phase systems, the forward and backward time integration leads to pre- and post-actuation intervals for the input trajectory. This means that the input $u^{*}(t)$ has to start in advance (noncausality) in order to realize the predefined output transition $y^{*}(t)$, and a post-steering is required after the output transition is finished (Graichen et al., 2005a).

Example 1.2. The first-order ODE (1.2) for $u^{*}(t)$ represents the unstable internal dynamics of the linear SISO system (1.1). Since the internal dynamics is only of first-order (i.e. no stable subsystem exists), a bounded input trajectory $u^{*}(t)$ is obtained if (1.2) is integrated in reverse-time $t \in(-\infty, T]$. This scenario is schematically shown in Figure 1.3 for a desired output transition $y^{*}(0)=y_{0}^{*} \rightarrow y^{*}(T)=y_{T}^{*}$ between the stationary outputs $y_{0}^{*}$ and $y_{T}^{*}$ in the time $t \in[0, T]$. The corresponding stationary input values are $u_{0}^{*}=\frac{b_{0}}{a_{0}} y_{0}^{*}$ and $u_{T}^{*}=\frac{b_{0}}{a_{0}} y_{T}^{*}$. Due to the backward integration of (1.2) with the initial condition $u^{*}(T)=u_{T}^{*}$, the feedforward control $u^{*}(t)$ reaches its stationary value $u_{0}^{*}$ only asymptotically for $t \rightarrow-\infty$.
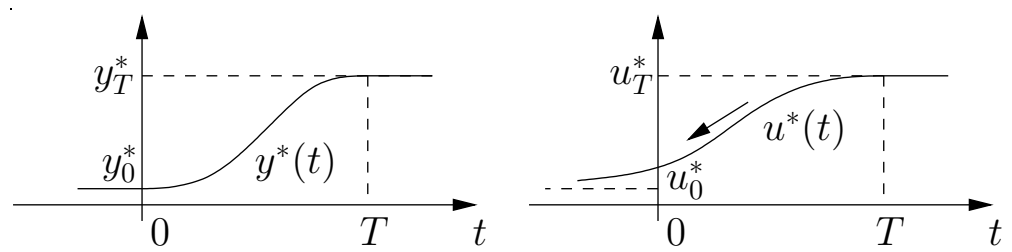

Figure 1.3: Input trajectory $u^{*}(t)$ determined by reverse-time integration of (1.2) in dependence of the desired output trajectory $y^{*}(t)$.

The effect of noncausality is well known and must be accepted if the emphasis of the feedforward control design is on tracking a predefined output trajectory $y^{*}(t)$. An alternative approach proposed by Benosman and Vey (2003) achieves causal and stable inversion of linear nonminimum-phase systems by planning - instead of predefining - an output trajectory such that the effect of the unstable zeros is canceled and no pre-actuation is necessary.

An algebraic framework for feedforward control design provides the notion of differential flatness introduced by Fliess, Lévine, Martin, and Rouchon (1995). The flatness property states that if a flat output exists, then all system variables as well as the input of the system can be parameterized in dependence of the flat output. Hence, the feedforward control design is purely algebraic, since the transition task can be defined in the coordinates of the flat output and the control trajectory is calculated without any time integration (Fliess and Marquez, 2000; Hagenmeyer and Zeitz, 2004a). If the flat output differs from the "real" output $y$, also the output trajectory $y^{*}(t)$ is obtained as parametrization by the flat output trajectory. The flatness-based feedforward control design is particularly convenient for linear systems, since all linear systems are flat if they are controllable (Rothfuss, 1997; Hagenmeyer and Zeitz, 2004a; Sira-Ramírez and Agrawal, 2004). 
Example 1.3. The output $y$ of the linear SISO example (1.1) is a flat output of the system if $b_{1}=0$ holds. In this case, the input trajectory $u^{*}(t)$ directly follows from the input-output relation (1.2) to

$$
\Sigma_{F F}: \quad u^{*}=\frac{1}{b_{0}}\left(a_{0} y^{*}+a_{1} \dot{y}^{*}+\ddot{y}^{*}\right)
$$

and can by algebraically determined without any time integration in dependence of a sufficiently smooth output trajectory $y^{*}(t) \in \mathcal{C}^{2}$.

A general problem of the inversion-based and flatness-based feedforward design is the fact that constraints (e.g. on the inputs and the states of the considered system) can only be incorporated indirectly, e.g. by choosing different types of setup functions for the output trajectory $y^{*}(t)$ or increasing the transition time. For instance, Piazzi and Visioli (2001) address the feedforward control design for setpoint transitions of linear scalar systems with smoothness constraints on the input and output trajectory. The constraints are considered by iteratively minimizing the transition time until the input and (predefined) output trajectory are tangent to the respective constraints, i.e. the trajectories do not actually hit and remain at the constraints. Moreover, the noncausality of the feedforward control in the case of nonminimum-phase linear systems cannot be avoided due to the predefinition of the output trajectory.

A numerical more demanding approach to account for general state constraints in the inversionbased or flatness-based feedforward control design is to (at least partially) express the states and inputs of the system in terms of the output and its time derivatives, which are parameterized by appropriate setup functions. Agrawal et al. (1999) considered the finite-time transition problem with state and input constraints for linear controllable systems, where the constraints can be reduced to a set of linear inequalities for the flat output and its time derivatives. Further results on constrained trajectory generation for nonlinear flat systems and an overview on this topic can be found in (von Löwis, 2002).

The feedforward control is further complicated if a cost functional has to be minimized in addition to the constraints, e.g. to minimize the energy for steering a system between two setpoints. In this case, the parameterization of the output and its time derivatives leads to an order-reduction of the original dynamic optimization problem, see e.g. (Petit et al., 2001) and (Oldenburg and Marquardt, 2002). Although these approaches reduce the complexity of optimization-based or constrained feedforward control design by parameterizing the output and its time derivatives, their solution - usually requiring optimization tools or (non)linear programming - is still numerically heavy.

\subsection{Goals and outline of the thesis}

The overview on feedforward control design shows that general difficulties exist with the inversion of non-flat and nonminimum-phase nonlinear systems due to the required numerical solution of the internal dynamics. This problem is directly linked to the fact that predefining 
the output trajectory $y^{*}(t)$ leads to pre- and/or post-actuation intervals if the output is not a flat output. On the other hand, predefining the output trajectory $y^{*}(t)$ is a strong restriction on the feedforward control design concerning the considered finite-time transition between stationary setpoints. The pre- and/or post-actuation intervals also lead to the problem that the stationary setpoints are only reached asymptotically and not in the desired finite time interval. Moreover, as mentioned in the last section, the feedforward control design is significantly complicated in the presence of constraints with heavy numerical load if optimization-based approaches or (non)linear programming are used.

In this thesis, the transition between two stationary setpoints is treated as a two-point BVP throughout all design steps of the inversion-based feedforward control in order to realize the setpoint transition in a finite time interval. With respect to the above discussion, the following questions arise concerning the feedforward control design:

- How can the difficulties connected to non-flat, nonminimum-phase, and nonlinear systems be overcome for finite-time transition problems?

- How can constraints on the feedforward control $u^{*}(t)$ and output trajectory $y^{*}(t)$ be systematically incorporated in the BVP formulation, in order to

- maintain numerical robustness of the feedforward control design,

- provide an easy-to-implement approach using standard BVP solvers.

- How can the inversion-based feedforward control design for finite-time transition problems be extended to nonlinear multiple-input multiple-output (MIMO) systems?

The goals of the thesis directly correspond to the structure of the chapters. In Chapter 2, the inversion-based feedforward control design is introduced for nonlinear SISO systems. Thereby, the finite-time transition problem is treated as a two-point BVP in the coordinates of the input-output normal form. The key role in the inversion-based design plays the BVP of the internal dynamics, which can be solved numerically with standard BVP solvers, e.g. with the MATLAB function bvp4c (Shampine et al., 2000). An illustrative example for the feedforward control design is the swing-up of the double pendulum on a cart with both numerical and experimental results. The derived model of the double pendulum is highly nonlinear and nonminimum-phase, thus showing the potential of the feedforward control design.

Chapter 3 addresses the feedforward control design under input constraints by extending the previous internal dynamics BVP by a further BVP for the output. Moreover, the transition time of the desired setpoint change can be determined as part of the BVP solution in dependence of a desired aggressiveness of the feedforward control $u^{*}(t)$. The feedforward control design under input constraints is illustrated for a setpoint change scenario of a continuous stirred tank reactor (CSTR).

In Chapter 4 , constraints on the output trajectory $y^{*}(t)$ and its time derivatives are systematically incorporated in a dynamic system by means of saturation functions and successive differentiation of the output. The newly constructed dynamic system replaces the previous output 
BVP. Its numerical solution yields the output trajectory which satisfies the constraints. In this way, the output constraints are directly incorporated in the formulation of the BVP for the finite-time transition problem. Moreover, the input constraints can be treated as constraints on the highest time derivative of the output. The concept is applied to the side-stepping problem of the triple inverted pendulum on a cart with constraints on the cart position, velocity, and acceleration. The experimental results show the accuracy of the feedforward control together with a stabilizing state feedback controller.

In Chapter 5, the results presented in the previous chapters for nonlinear SISO systems are extended to the MIMO case. Similar to the SISO case, the finite-time transition problem is treated as a two-point BVP in the coordinates of the MIMO input-output normal form. The constraints on the multiple input and output channels can be incorporated by adapting the results for nonlinear SISO systems. The feedforward control design is illustrated for a flight maneuver of the 3DOF helicopter (Kiefer et al., 2006) with constraints on both inputs and the pitch angle by providing numerical and experimental results. Finally, the results of the thesis are summarized in Chapter 6 and an outlook on potential future work is given.

Appendix A summaries different setups of the output trajectory with free parameters for the feedforward control design in the unconstrained case (cf. Chapter 2). Appendix B describes different methods for the numerical solution of two-point BVPs. Appendix C extends the results of Chapter 4 concerning the incorporation of output constraints within the feedforward control design. It is shown that the constructed dynamic system for the constrained output is also valid for non-asymptotic saturation functions such that the output constraints are exactly (non-asymptotically) fulfilled. 


\section{Chapter 2}

\section{Feedforward control design for nonlinear SISO systems}

This chapter presents the inversion-based feedforward control design for nonlinear SISO systems with application to finite-time transitions between stationary setpoints. The finite-time transition problem is treated as a two-point boundary value problem (BVP) throughout all design steps of the feedforward design. The basis for the inversion-based design is the inputoutput normal form of the considered system, where the numerical solution of the BVP of the internal dynamics plays the key role in the feedforward control design. In this context, the inversion-based approach corresponds to the flatness-based feedforward design if the output is a flat output of the system, since in this case no internal dynamics exists and the feedforward control is obtained purely algebraically. The potential of the inversion-based feedforward design is demonstrated for the swing-up of the double pendulum as finite-time transition problem.

\subsection{Finite-time transition between stationary setpoints}

Considered are nonlinear SISO systems

$$
\begin{aligned}
\Sigma: \quad \dot{\boldsymbol{x}} & =\boldsymbol{f}(\boldsymbol{x}, u), \quad \boldsymbol{x}(0)=\boldsymbol{x}_{0}, \\
y & =h(\boldsymbol{x})
\end{aligned}
$$

with time $t \in \mathbb{R}$, state $\boldsymbol{x} \in \mathbb{R}^{n}$, input $u \in \mathbb{R}$, and output $y \in \mathbb{R}$. The vector field $\boldsymbol{f}: \mathbb{R}^{n} \times \mathbb{R} \rightarrow \mathbb{R}^{n}$ and the output function $h: \mathbb{R}^{n} \rightarrow \mathbb{R}$ are assumed to be sufficiently smooth.

A widespread control problem concerns the transition between two stationary setpoints $\left(u_{0}^{*}, \boldsymbol{x}_{0}^{*}, y_{0}^{*}\right)$ and $\left(u_{T}^{*}, \boldsymbol{x}_{T}^{*}, y_{T}^{*}\right)$ of system $(2.1)$ within a finite time interval $t \in[0, T]$. It is assumed that the stationary solutions

$$
\begin{array}{lrl}
\left(\boldsymbol{x}_{0}^{*}, u_{0}^{*}\right): & \boldsymbol{f}\left(\boldsymbol{x}_{0}^{*}, u_{0}^{*}\right)=\mathbf{0}, & y_{0}^{*}=h\left(\boldsymbol{x}_{0}^{*}\right), \\
\left(\boldsymbol{x}_{T}^{*}, u_{T}^{*}\right): & \boldsymbol{f}\left(\boldsymbol{x}_{T}^{*}, u_{T}^{*}\right)=\mathbf{0}, & y_{T}^{*}=h\left(\boldsymbol{x}_{T}^{*}\right) .
\end{array}
$$


are uniquely determined. The transition between the setpoints (2.2) in a finite time $T$ imposes the following boundary conditions (BCs) on the system (2.1):

$$
\boldsymbol{x}(0)=\boldsymbol{x}_{0}^{*}, \quad \boldsymbol{x}(T)=\boldsymbol{x}_{T}^{*},
$$

whereby the initial state $\boldsymbol{x}_{0}=\boldsymbol{x}_{0}^{*}$ has to be consistent with the stationary setpoint $\boldsymbol{x}_{0}^{*}$. From a mathematical point of view, the $n$ first-order ordinary differential equations (ODEs) in (2.1) and the $2 n$ BCs (2.3) form a two-point boundary value problem (BVP) for the states $\boldsymbol{x}=\left[x_{1}, \ldots, x_{n}\right]^{\top}$ in dependence of the input trajectory $u(t), t \in[0, T]$. Obviously, some controllability properties are required for the system (2.1) to accomplish the finite-time transition. The determination of a feedforward control $u^{*}(t), t \in[0, T]$ steering the system from the initial state $\boldsymbol{x}_{0}^{*}$ to the terminal state $\boldsymbol{x}_{T}^{*}$ is the main objective of the feedforward control design.

In view of the open-loop control scheme in Figure 1.2, the determination of the feedforward control trajectory $u^{*}(t)$ is strongly related to the design of the desired output trajectory $y^{*}(t)$, which is provided by the signal generator $\Sigma^{*}$. The trajectory $y^{*}(t)$ must appropriately connect the initial and terminal values $y_{0}^{*}$ and $y_{T}^{*}$ given in (2.2). Figure 2.1 shows an example of a respective trajectory $y^{*}(t)$ which is also used as reference for the output feedback control $\Sigma_{F B}$ in Figure 1.1. Thereby, the transition time $T$ is an important design parameter which has to be chosen appropriately with respect to the system dynamics and possibly given constraints.

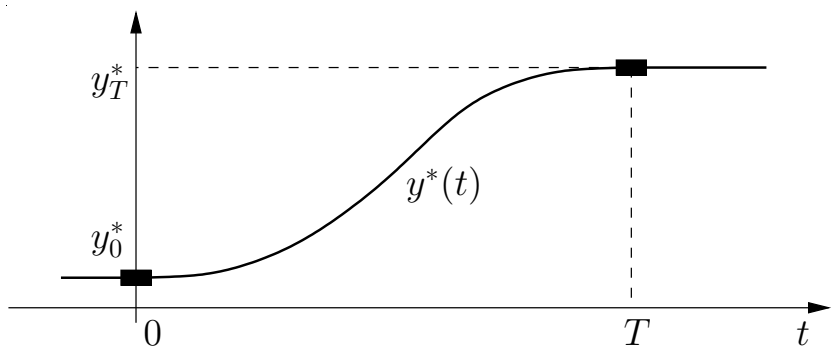

Figure 2.1: Desired output trajectory $y^{*}(t)$ for the transition between the stationary setpoints $y^{*}(0)=$ $y_{0}^{*}$ and $y^{*}(T)=y_{T}^{*}$ within the time interval $t \in[0, T]$.

\subsubsection{Transition problem in flat coordinates}

The feedforward control design is straightforward if the considered SISO system (2.1) is differentially flat (Fliess et al., 1995). The flatness property states that if there exists a flat output $z$, the state $\boldsymbol{x}$ as well as the input $u$ can be parameterized in dependence of $z$ :

$$
\begin{aligned}
z & =A(\boldsymbol{x}) \\
\boldsymbol{x} & =\boldsymbol{B}\left(z, \dot{z}, \ldots, z^{(n-1)}\right), \\
u & =C\left(z, \dot{z}, \ldots, z^{(n)}\right) .
\end{aligned}
$$

The parameterization of $\boldsymbol{x}$ and $u$ in terms of the flat output $z$ and its time derivatives illustrates that the feedforward control design is purely algebraic if the transition problem $(2.2)-(2.3)$ is 
defined in the coordinates of the flat output. The stationary setpoints (2.2) correspond to the stationary values

$$
z_{0}^{*}=A\left(\boldsymbol{x}_{0}^{*}\right), \quad z_{T}^{*}=A\left(\boldsymbol{x}_{T}^{*}\right)
$$

for the flat output (2.4). By planning a sufficiently smooth flat output trajectory $z^{*}(t) \in \mathcal{C}^{n}, t \in$ $[0, T]$ with the boundary conditions ${ }^{1}$

$$
z^{*}(0)=z_{0}^{*}, \quad z^{*}(T)=z_{T}^{*}, \quad z^{*(i)}(0)=z^{*(i)}(T)=0, \quad i=1, \ldots, n,
$$

the feedforward control $u^{*}(t)$ and state trajectory $\boldsymbol{x}^{*}(t), t \in[0, T]$ can be algebraically calculated by means of (2.5) and (2.6):

$$
\begin{aligned}
\boldsymbol{x}^{*} & =\boldsymbol{B}\left(z^{*}, \dot{z}^{*}, \ldots, z^{*(n-1)}\right), \\
u^{*} & =C\left(z^{*}, \dot{z}^{*}, \ldots, z^{*(n)}\right) .
\end{aligned}
$$

Finally, the "real" output trajectory $y^{*}(t), t \in[0, T]$ in $(2.1)$ can be determined in dependence of the flat output trajectory $z^{*}$ and its time derivatives up to order $n-r$ (Hagenmeyer and Zeitz, 2004b):

$$
y^{*}=D\left(z^{*}, \dot{z}^{*}, \ldots, z^{*(n-r)}\right) .
$$

The feedforward control $u^{*}(t)$ and the desired output trajectory $y^{*}(t)$ have to be checked with respect to constraints, whereby the shape of $u^{*}(t)$ and $y^{*}(t)$ can be influenced by the choice of setup function for the flat output $z^{*}(t)$ (e.g. polynomials or spline functions) or by altering the transition time $T$.

Example 2.1 (Example 1.1 continued). Consider again the linear SISO system (1.1) with $b_{0} \neq 0$. For the flatness-based feedforward control design, the transfer function (1.1) can be written in the coordinates of the controller normal form of order $n=2$ (Hagenmeyer and Zeitz, 2004b)

$$
\Sigma: \quad \boldsymbol{x}=\left[\begin{array}{cc}
0 & 1 \\
-a_{0} & -a_{1}
\end{array}\right] \boldsymbol{x}+\left[\begin{array}{l}
0 \\
1
\end{array}\right] u, \quad y=\left[b_{0}-b_{1}\right] \boldsymbol{x} .
$$

The system (2.12) exhibits the flat output $z=x_{1}$ with the state vector $\boldsymbol{x}=[z, \dot{z}]^{\top}$ comprising $z$ and its time derivative $\dot{z}$. For a desired setpoint change between the stationary outputs $y^{*}(0)=y_{0}^{*} \rightarrow y^{*}(T)=y_{T}^{*}$, the corresponding stationary values of the flat output $z$ follow from (2.12) to $z_{0}^{*}=y_{0}^{*} / b_{0}$ and $z_{T}^{*}=y_{T}^{*} / b_{0}$. A flat output trajectory $z^{*}(t) \in \mathcal{C}^{2}$ is constructed with the polynomial

$$
z^{*}(t)=z_{0}^{*}+\left(z_{T}^{*}-z_{0}^{*}\right)\left[10\left(\frac{t}{T}\right)^{3}-15\left(\frac{t}{T}\right)^{4}+6\left(\frac{t}{T}\right)^{5}\right], \quad t \in[0, T]
$$

satisfying the BCs (2.8) for $n=2$. The parameterization of the feedforward control $u^{*}(t)$ and output trajectory $y^{*}(t)$ can be derived from (2.12):

$$
u^{*}=a_{0} z^{*}+a_{1} \dot{z}^{*}+\ddot{z}^{*}, \quad y^{*}=b_{0} z^{*}-b_{1} \dot{z}^{*}, \quad t \in[0, T] .
$$

\footnotetext{
${ }^{1}$ The homogeneous BCs in (2.8) for $z^{(n)}$ ensure that the feedforward control $u^{*}(t)$ is continuous at the bounds $t=0$ and $t=T$.
} 
Figure 2.2 shows the nominal trajectories $z^{*}(t), y^{*}(t)$, and $u^{*}(t)$ for the setpoint transition between $y_{0}^{*}=0$ and $y_{T}^{*}=1$ with the parameters

$$
a_{0}=1, \quad a_{1}=2, \quad b_{0}=4, \quad b_{1}=1
$$

and three different (dimensionless) transition times $T$. The output $y^{*}(t)$ reveals an initial undershoot which is characteristic for nonminimum-phase systems. The trajectories furthermore show the influence of the transition time $T$ on the amplitude of the feedforward control $u^{*}(t)$ and output trajectory $y^{*}(t)$.
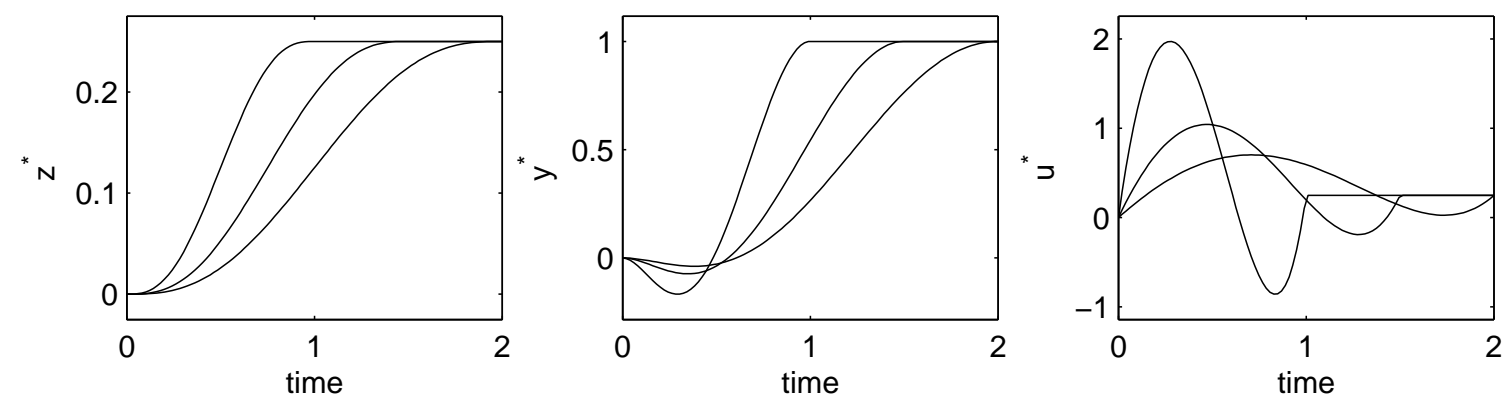

Figure 2.2: Flatness-based feedforward design for the setpoint transition $y_{0}^{*}=0 \rightarrow y_{T}^{*}=1$ of the linear SISO system (1.1) with parameters (2.15) for various transition times $T \in\{1,1.5,2\}$.

Although the flatness methodology allows an algebraic and convenient design of the feedforward control, there are also some issues which are worth mentioning. The parameterizations (2.5)(2.6) of the state $\boldsymbol{x}$ and input $u$ are often described by large analytic expressions, especially if the considered system is highly nonlinear, e.g. as in case of polymerization reactors (Rothfuss et al., 1996). Furthermore, the derivation of (2.5)-(2.6) by symbolic calculations might lead to singularities (e.g. in denominator expressions) which have to be accounted for, either by avoiding to pass through the singularity or otherwise by appropriately planning the flat output trajectory $z^{*}(t)$ or applying a time scaling to steer through the singularity (Martin et al., 1997; Lenoir et al., 1998).

\subsubsection{Transition problem in input-output coordinates}

If the considered system (2.1) is not flat or at least a flat output cannot be found, the flatnessbased feedforward design cannot be applied and the setpoint transition has to be defined with respect to the "real" output $y$. This is also necessary if constraints for the output $y$ have to be incorporated in the feedforward control design, as described in Chapter 4. Moreover, even if the system is flat, it may be advantageous to define the transition problem in dependence of the output $y$ instead of using the flat output $z$, in order to avoid the above-mentioned occurrence of singularities or to reduce the complexity of the analytic expressions.

The derivation of the input-output representation for the SISO system (2.1) is based on the following definition of the relative degree $r$. 
Definition 2.1. The nonlinear SISO system (2.1) has the relative degree $r$ at point $\boldsymbol{x}^{0}$ if the following condition is fulfilled (Isidori, 1995; Nijmeijer and van der Schaft, 1990)

$$
\frac{\partial}{\partial u} L_{\boldsymbol{f}}^{i} h=0, \quad i \in\{0, \ldots, r-1\}, \quad \frac{\partial}{\partial u} L_{f}^{r} h \neq 0
$$

for all admissible $u$ and all $\boldsymbol{x}$ in a neighborhood of $\boldsymbol{x}^{0}$. The operator $L_{\boldsymbol{f}}$ represents the Lie derivative along the vector field $\boldsymbol{f}$.

Literally, the relative degree $r$ with $0<r \leq n$ denotes how often the output $y$ has to be differentiated until the input $u$ appears explicitly. ${ }^{2}$ In the following, it is assumed that the relative degree $r$ is well-defined at least locally, i.e. $r$ is independent of the state $\boldsymbol{x}$.

The input-output coordinates of the system (2.1) are determined via the diffeomorphism (Isidori, 1995; Nijmeijer and van der Schaft, 1990)

$$
\left[y, \dot{y}, \ldots, y^{(r-1)}, \boldsymbol{\eta}^{\top}\right]^{\top}=\phi(\boldsymbol{x})
$$

with the output derivatives

$$
y^{(i)}=L_{\boldsymbol{f}}^{i} h(\boldsymbol{x})=\phi_{i+1}(\boldsymbol{x}), \quad i=0, \ldots, r-1
$$

and the supplementary state vector

$$
\boldsymbol{\eta}=\phi_{\eta}(\boldsymbol{x}) \in \mathbb{R}^{n-r}
$$

to complete the diffeomorphism $\phi(\boldsymbol{x}) \in \mathbb{R}^{n}$ to a coordinate system. By means of the coordinates (2.17), the SISO system (2.1) is transformed into the nonlinear input-output normal form

$$
\begin{aligned}
\Sigma_{I O}: \quad y^{(r)} & =\alpha\left(y, \dot{y}, \ldots, y^{(r-1)}, \boldsymbol{\eta}, u\right), \\
\dot{\boldsymbol{\eta}} & =\boldsymbol{\beta}\left(y, \dot{y}, \ldots, y^{(r-1)}, \boldsymbol{\eta}, u\right)
\end{aligned}
$$

with $\alpha(\cdot)=L_{\boldsymbol{f}}^{r} h \circ \boldsymbol{\phi}^{-1}$ and $\beta_{i}(\cdot)=L_{\boldsymbol{f}} \phi_{\eta, i} \circ \boldsymbol{\phi}^{-1}, i=1, \ldots, n-r$. The above assumption concerning the well-defined relative degree $r$ implies that $\partial \alpha / \partial u \neq 0$ holds at least locally. The chain of $r$ integrators (2.18) with input $u$ represents the input-output dynamics. The internal dynamics is defined by the differential equation (2.19) for the state $\boldsymbol{\eta} \in \mathbb{R}^{n-r}$. In the special case $r=n$, no internal dynamics (2.19) exists and $y$ represents a flat output of the system (2.1) (Fliess et al., 1995; Hagenmeyer and Zeitz, 2004b).

The BCs (2.3) of the considered finite-time transition problem can be transformed via the diffeomorphism (2.17) into the input-output coordinates:

$$
\begin{array}{ll}
y(0)=y_{0}^{*}=h\left(\boldsymbol{x}_{0}^{*}\right), & y(T)=y_{T}^{*}=h\left(\boldsymbol{x}_{T}^{*}\right),\left.\quad y^{(i)}\right|_{t=0, T}=0, \quad i=1, \ldots, \rho \in\{r-1, r\} \\
\boldsymbol{\eta}(0)=\boldsymbol{\eta}_{0}^{*}=\boldsymbol{\phi}_{\eta}\left(\boldsymbol{x}_{0}^{*}\right), & \boldsymbol{\eta}(T)=\boldsymbol{\eta}_{T}^{*}=\boldsymbol{\phi}_{\eta}\left(\boldsymbol{x}_{T}^{*}\right) .
\end{array}
$$

\footnotetext{
${ }^{2}$ The original definition of the relative degree $r$ in (Isidori, 1995; Nijmeijer and van der Schaft, 1990) is based on the input-affine representation of a nonlinear SISO system, but is adapted here to the general nonlinear system (2.1).
} 
Thereby, the original BVP (2.1)-(2.3) is split into two coupled BVPs (2.18)-(2.21) for $y(t)$ and $\boldsymbol{\eta}(t)$ in dependence of the input $u(t), t \in[0, T]$. The symbol $\rho$ in $(2.20)$ denotes the number of derivatives $y^{(i)}$ with homogeneous BCs. Thereby, the value $\rho=r-1$ corresponds to the number of output coordinates defined in (2.17). However, if the input $u(t)$ has to be continuous at the bounds $t=0$ and $t=T$, the inversion of (2.18) with respect to $u$ shows that the highest output derivative $y^{(r)}$ has to satisfy the additional BCs $y^{(r)}(0)=y^{(r)}(T)=0$ denoted by $\rho=r$ in $(2.20)$.

Example 2.2 (Example 1.1 continued). The relative degree $r$ of the linear SISO system (1.1) is the difference degree between denominator and numerator polynomial, i.e. $r=2-1=1$. Its input-output normal form can be written as ${ }^{3}$ (Zeitz et al., 2005)

$$
\begin{aligned}
& \dot{y}=-a_{1} y+\eta-b_{1} u=\alpha(y, \eta, u), \\
& \dot{\eta}=-a_{0} y+b_{0} u \quad=\beta(y, u) .
\end{aligned}
$$

For a transition between the stationary setpoints $\left(y_{0}^{*}, \eta^{*}\right) \rightarrow\left(y_{T}^{*}, \eta_{T}^{*}\right)$ within the finite time interval $t \in[0, T]$, the $B C s(2.20)-(2.21)$ read

$$
\begin{aligned}
& y(0)=y_{0}^{*}, \quad y(T)=y_{T}^{*},\left.\quad \dot{y}^{*}\right|_{t=0, T}=0 \\
& \eta(0)=\eta_{0}^{*}=a_{1} y_{0}^{*}+b_{1} u_{0}^{*}, \quad \eta(T)=\eta_{T}^{*}=a_{1} y_{T}^{*}+b_{1} u_{T}^{*},
\end{aligned}
$$

whereby the stationary input values $u_{0}^{*}=\frac{a_{0}}{b_{0}} y_{0}^{*}$ and $u_{T}^{*}=\frac{a_{0}}{b_{0}} y_{T}^{*}$ follow from (2.23) with $\dot{\eta}=0$.

\subsection{Inversion-based feedforward control design}

The determination of the feedforward trajectory $u^{*}(t)$ requires the solution of the two coupled BVPs (2.18)-(2.21). The inversion-based feedforward control design (Devasia et al., 1996; Chen and Paden, 1996; Graichen et al., 2005a) is based on the inverse

$$
u^{*}=\alpha^{-1}\left(y^{*}, \ldots, y^{*(r)}, \boldsymbol{\eta}^{*}\right)
$$

of the input-output dynamics (2.18) that depends on the desired output trajectory $y^{*}(t)$ and the state $\boldsymbol{\eta}^{*}(t)$ of the internal dynamics (2.19). ${ }^{4}$ The output trajectory $y^{*}(t) \in \mathcal{C}^{\rho}$ has to satisfy the BCs (2.20), whereby $\rho=r$ holds if continuity of the feedforward control (2.26) is desired.

\footnotetext{
${ }^{3}$ The input-output normal form (2.22)-(2.23) can be derived by dividing the input-output relation of (1.1) by the Laplace variable $s: s Y(s)=-a_{1} Y(s)-b_{1} U(s)+\left(-a_{0} Y(s)+b_{0} U(s)\right) s^{-1}$. This ODE represents the inputoutput dynamics, whereby substituting the last term by $\eta(s)=\left(-a_{0} Y(s)+b_{0} U(s)\right) s^{-1}$ yields the internal dynamics of the linear SISO system (1.1). Alternatively, the controller normal form (2.12) with the coordinates $\boldsymbol{x}$ can be used to determine the diffeomorphism $(2.17)$, i.e. $[y, \eta]^{\top}=\boldsymbol{\phi}(\boldsymbol{x})$, with an appropriate choice of $\eta$.

${ }^{4}$ The asterisk $\left(^{*}\right)$ is used to characterize the feedforward variables.
} 


\subsubsection{Boundary value problem of the internal dynamics}

In the special case $r=n$, the output $y$ represents a flat output of the system (2.1), i.e. $z=y$ in Section 2.1.1, such that the calculation of the feedforward control (2.26) would be purely algebraic in absence of the internal dynamics state $\boldsymbol{\eta}^{*}(t)$. For the general case $r<n$ however, the determination of the feedforward control $u^{*}(t)$ requires the solution of the internal dynamics. Replacing the input $u$ in (2.19) by the feedforward control (2.26) yields

$$
\dot{\boldsymbol{\eta}}^{*}=\overline{\boldsymbol{\beta}}\left(\boldsymbol{\eta}^{*}, y^{*}, \dot{y}^{*}, \ldots, y^{*(r)}\right)
$$

with the output trajectory $y^{*}(t)$ and its first $r$ time derivatives serving as input to $(2.27) .^{5}$ The zero dynamics of the system (2.1) is obtained by zeroing the output and its time derivatives $y^{*(i)}=0, i=0,1, \ldots, r$ in (2.27). If the zero dynamics is stable, the system (2.1) is minimumphase; otherwise it is nonminimum-phase.

The state $\boldsymbol{\eta}^{*}(t)$ has to satisfy the BCs $(2.21)$

$$
\boldsymbol{\eta}^{*}(0)=\boldsymbol{\eta}_{0}^{*}, \quad \boldsymbol{\eta}^{*}(T)=\boldsymbol{\eta}_{T}^{*}
$$

in order to steer the internal dynamics from the initial state $\boldsymbol{\eta}_{0}^{*}$ to the terminal state $\boldsymbol{\eta}_{T}^{*}$. Thereby, the resulting BVP (2.27)-(2.28) is overdetermined by $2(n-r)$ BCs for $n-r$ ODEs. Its solution technique plays the key role in the feedforward control design.

In the stable inversion approach of Devasia et al. (1996) and Chen and Paden (1996), the output trajectory $y^{*}(t)$ is predefined and the internal dynamics $(2.27)$ is solved with an iterative forward/backward integration scheme in dependence of its stability properties. ${ }^{6}$ In this case, the output trajectory $y^{*}(t), t \in[0, T]$ exactly fulfills the BCs $(2.20)$ for the setpoint transition, but $\boldsymbol{\eta}^{*}(t)$ reaches one or both BCs in (2.28) only asymptotically for $t \rightarrow-\infty$ or $t \rightarrow \infty$, which leads to pre- and/or post-actuation intervals for the feedforward control $u^{*}(t)$ (Devasia et al., 1996; Chen and Paden, 1996; Graichen et al., 2005a), also see Figure 1.3. In the case of pre-actuation, the feedforward control is referred to as being noncausal.

As a result, the transition between the setpoints (2.2) is not performed in the finite time interval $t \in[0, T]$. Moreover, the theoretically infinite pre- and post-actuation intervals $(-\infty, 0]$ and $[T, \infty)$ can only be realized approximately, whereby the accuracy of the feedforward control $u^{*}(t)$ depends on the length of the actually implemented pre- and post-actuation (Zou and Devasia, 2004).

These points reveal the importance of appropriately planning the desired output trajectory $y^{*}(t)$ in course of the feedforward control design. The calculation of a feedforward control $u^{*}(t)$ for a predefined output trajectory $y^{*}(t)$ is of importance for applications where exact output

\footnotetext{
${ }^{5}$ This new representation of the internal dynamics is often referred to as tracking dynamics, see e.g. (Marino and Tomei, 1995).

${ }^{6}$ A detailed description of the stable inversion algorithm can be found in (Tomlin and Sastry, 1997). Its implementation using finite-difference methods instead of the iterative forward/backward integration is addressed in (Taylor and Li, 2002).
} 
tracking is required (see Table 1.1). However, if a finite-time transition between two setpoints is desired, the predefinition of an output trajectory $y^{*}(t)$ represents a strong restriction on the feedforward control design.

\subsubsection{Output trajectory with free parameters}

From a mathematical point of view, the BVP (2.27)-(2.28) requires $n-r$ additional degrees of freedom in order to exactly fulfill the BCs (2.28) (Graichen et al., 2005a). Therefore, $n-r$ free parameters $\boldsymbol{p}=\left(p_{1}, \ldots, p_{n-r}\right)$ are provided in a setup function

$$
y^{*}(t)=\Upsilon(t, \boldsymbol{p})
$$

for the output trajectory $y^{*}(t)$, which has to satisfy the output BCs $(2.20)$

$$
\Upsilon(0, \boldsymbol{p})=y_{0}^{*}, \quad \Upsilon(T, \boldsymbol{p})=y_{T}^{*},\left.\quad \Upsilon^{(i)}\right|_{t=0, T}=0, \quad i=1, \ldots, \rho \in\{r-1, r\} .
$$

As mentioned before, $\Upsilon(t, \boldsymbol{p}) \in \mathcal{C}^{\rho}$ has to be $\rho=r$ times differentiable if continuity of the input $u^{*}(t)$ is desired, otherwise $\Upsilon(t, \boldsymbol{p}) \in \mathcal{C}^{\rho}$ with $\rho=r-1$ is sufficient. Two suitable choices for the construction of $\Upsilon(t, \boldsymbol{p}) \in \mathcal{C}^{\rho}$ are the following polynomial and cosine series

$$
\Upsilon(t, \boldsymbol{p})= \begin{cases}y_{0}^{*}+\sum_{i=\rho+1}^{2 \rho+1} a_{i}\left(\frac{t}{T}\right)^{i}+\sum_{i=1}^{n-r} p_{i}\left(\frac{t}{T}\right)^{i+2 \rho+1} & \text { polynomial } \\ \sum_{i=0}^{\bar{\rho}+1} a_{i} \cos \left(\frac{i \pi t}{T}\right)+\sum_{i=1}^{n-r} p_{i} \cos \left(\frac{(\bar{\rho}+1+i) \pi t}{T}\right) & \text { cosine series }\end{cases}
$$

In the polynomial setup (2.31a), the free parameters $p_{i}, i=1, \ldots, n-r$ are the coefficients of the highest order terms. The particular structure of (2.31a) ensures that the BCs (2.30) for $t=0$ are satisfied, which is easily verified by differentiating (2.31a) and inserting $t=0$. The coefficients $a_{i}, i=\rho+1, \ldots, 2 \rho+1$ are determined by solving the set of equations stemming from the BCs (2.30) at $t=T$.

The cosine series (2.31b) uses the free parameters $p_{i}, i=1, \ldots, n-r$ as the coefficients of the cosine terms with the highest frequencies. A characteristic property of the cosine series $(2.31 \mathrm{~b})$ is that the $\mathrm{BCs}(2.30)$ for odd time derivatives $i=1,3, \ldots$ are directly satisfied due to the sine terms appearing in the respective odd derivatives of $(2.31 \mathrm{~b})$. The remaining coefficients $a_{i}, i=0,1, \ldots, \bar{\rho}+1$ with

$$
\bar{\rho}= \begin{cases}\rho & \text { if } \rho \text { even } \\ \rho-1 & \text { if } \rho \text { odd }\end{cases}
$$

have to be determined such that the $\bar{\rho}+2$ BCs in $(2.30)$ for $\Upsilon^{(i)}(t, \boldsymbol{p})$ with the even numbers $i=0,2, \ldots, \bar{\rho}$ are satisfied.

For both the polynomial (2.31a) and the cosine series (2.31b), the coefficients $a_{i}$ depend on the free parameters $\boldsymbol{p}$ in order to satisfy the respective BCs in (2.30). Appendix A summarizes the coefficients $a_{i}$ of both setups (2.31a) and (2.31b) for the first four continuous functions $\Upsilon(t, \boldsymbol{p}) \in$ $\mathcal{C}^{\rho}, \rho=1, \ldots, 4$ and various numbers of free parameters $\boldsymbol{p}=\left(p_{1}, \ldots, p_{q}\right)$ with $q=n-r$. 
Remark 2.1. The cosine series is often numerically more robust for higher-order systems than the polynomial (2.31a), since the polynomial coefficients increase significantly in magnitude for higher-order terms. On the other hand, the cosine series may be too smooth for many applications, e.g. if only $C^{2}$-continuity is required for the output $y^{*}(t)$, but the cosine series is $\mathcal{C}^{3}$-continuous. This often leads to higher magnitudes of the output and input trajectories $y^{*}(t)$ and $u^{*}(t)$ in order to compensate the smoother start and stop of the output transition.

Placing the output trajectory $y^{*}(t)=\Upsilon(t, \boldsymbol{p})$ into the BVP $(2.27)-(2.28)$ of the internal dynamics yields the 2-point BVP for the state $\boldsymbol{\eta}^{*}(t)$ with the free parameters $\boldsymbol{p}$ :

$$
\dot{\boldsymbol{\eta}}^{*}=\overline{\boldsymbol{\beta}}\left(\boldsymbol{\eta}^{*}, \Upsilon(t, \boldsymbol{p}), \dot{\Upsilon}(t, \boldsymbol{p}), \ldots, \Upsilon^{(r)}(t, \boldsymbol{p})\right), \quad \boldsymbol{\eta}^{*}(0)=\boldsymbol{\eta}_{0}^{*}, \quad \boldsymbol{\eta}^{*}(T)=\boldsymbol{\eta}_{T}^{*}
$$

The parameters $\boldsymbol{p}=\left(p_{1}, \ldots, p_{n-r}\right)$ provide the $n-r$ degrees of freedom for the solvability of the internal dynamics BVP. Its solution comprises the trajectory $\boldsymbol{\eta}^{*}(t), t \in[0, T]$ as well as the parameter set $\boldsymbol{p}$, which yields the desired output trajectory (2.29). The shape of $y^{*}(t)$ is determined by the parameter set $\boldsymbol{p}$ in $\Upsilon(t, \boldsymbol{p})$, which may result e.g. in an initial under- or overshoot occurring in the trajectory $y^{*}(t)$. This can be seen as a distortion of a predefined monotonic transition trajectory $y^{*}(t)$, which must be accepted to realize the finite-time transition (Graichen et al., 2005a). Finally, the feedforward control follows from (2.26) with the output (2.29) and $\boldsymbol{\eta}^{*}(t)$ :

$$
u^{*}=\alpha^{-1}\left(\Upsilon(t, \boldsymbol{p}), \ldots, \Upsilon^{*(r)}(t, \boldsymbol{p}), \boldsymbol{\eta}^{*}\right), \quad t \in[0, T] .
$$

In contrast to the stable system inversion (Devasia et al., 1996; Chen and Paden, 1996) mentioned in Section 2.2.1, the solution of the BVP (2.32) yields the trajectory of the internal dynamics $\boldsymbol{\eta}^{*}(t)$ such that the boundary points $\boldsymbol{\eta}_{0}^{*}$ and $\boldsymbol{\eta}_{T}^{*}$ are met for $t=0$ and $t=T$. Moreover, the input trajectory in (2.33) is causal, since $u^{*}(t)$ is constant for $t \notin[0, T]$, i.e. outside the transition interval.

Remark 2.2. Since the shape of the output trajectory $y^{*}(t)$ is not predefined but determined by the setup (2.31) in order to solve the BVP (2.32), a different output choice $\tilde{y}^{*}$ may be acceptable to achieve a well-defined relative degree $r$ or to reduce the complexity of the analytic expressions.

Example 2.3 (Example 2.2 continued). The inverse input-output dynamics (2.22) of the linear SISO system (1.1) yields the feedforward control (2.26)

$$
u^{*}=-\frac{1}{b_{1}}\left(a_{1} y^{*}+\dot{y}^{*}-\eta^{*}\right)=\alpha^{-1}\left(y^{*}, \dot{y}^{*}, \eta^{*}\right) .
$$

The BVP (2.27)-(2.28) is derived by inserting the input $u^{*}(t)$ into the internal dynamics (2.23)

$$
\dot{\eta}^{*}=-a_{0} y^{*}-\frac{b_{0}}{b_{1}}\left(a_{1} y^{*}+\dot{y}^{*}-\eta^{*}\right)=\bar{\beta}\left(\eta^{*}, y^{*}, \dot{y}^{*}\right), \quad \eta^{*}(0)=\eta_{0}^{*}, \quad \eta^{*}(T)=\eta_{T}^{*}
$$

subject to the BCs (2.25). Note that the linear internal dynamics is unstable, which corresponds to the zero of the transfer function (1.1) lying in the right half plane for $b_{0}, b_{1}>0$. The BVP 
(2.35) is overdetermined by 2 BCs for 1 ODE. Hence, one free parameter $p$ is required in the setup (2.29) of the output trajectory $y^{*}(t)$. The polynomial setup (2.31a) is used to construct a $\mathcal{C}^{1}$-continuous setup function

$$
\Upsilon(t, p)=y_{0}^{*}+a_{2}\left(\frac{t}{T}\right)^{2}+a_{3}\left(\frac{t}{T}\right)^{3}+p\left(\frac{t}{T}\right)^{4} .
$$

The coefficients $a_{2}=3\left(y_{T}^{*}-y_{0}^{*}\right)+p$ and $a_{3}=-2\left(y_{T}^{*}-y_{0}^{*}\right)-2 p$ (see Table A.1 in Appendix A.1) ensure that the BCs (2.30) for $\rho=1$ are satisfied. Figure 2.3 illustrates how the shape of the output trajectory $y^{*}(t)=\Upsilon(t, p)$ and its time derivative $\dot{y}^{*}(t)=\dot{\Upsilon}(t, p)$ are influenced by different parameter values $p$, while the stationary boundary values $y_{0}^{*}$ and $y_{T}^{*}$ are exactly met.
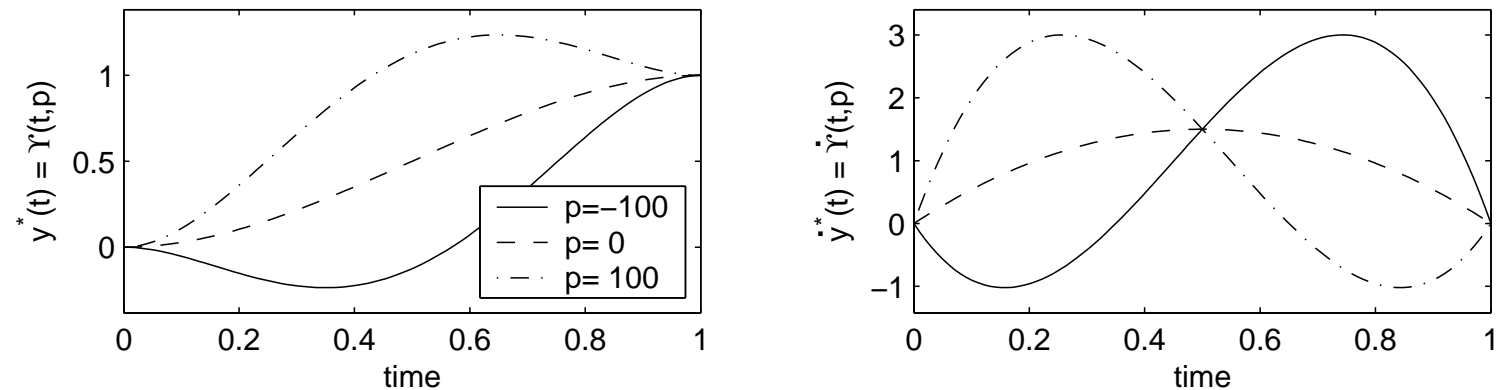

Figure 2.3: Output trajectory $y^{*}(t)=\Upsilon(t, p) \in \mathcal{C}^{1}$ in (2.36) for a setpoint transition $y_{0}^{*}=0 \rightarrow y_{T}^{*}=1$ and the transition time $T=1$ with various parameters $p$.

Remark 2.3. An alternative to providing $n-r$ free parameters $\boldsymbol{p}$ in the setup function $y^{*}(t)=$ $\Upsilon(t, \boldsymbol{p})$ seems to be to treat the transition time $T$ as a free parameter (via time transformation) and to provide $n-r-1$ free parameters in the set $\boldsymbol{p}$ of the setup function $\Upsilon(t, \boldsymbol{p})$. However, in general this will result in an ill-posed BVP, which can be illustrated for the linear SISO system in Example 2.3. The overdetermined internal dynamics $B V P(2.35)$ requires $n-r=1$ free parameter. If the transition time $T$ is considered as free parameter, the setup function (2.36) contains no parameter (by setting $p=0$ ), which yields a monotonic output trajectory $y^{*}=\Upsilon(t)$. Hence, corresponding to the stable inversion technique (Devasia et al., 1996; Chen and Paden, 1996), the unstable internal dynamics BVP (2.32) can be solved by a reverse-time integration starting at $\eta^{*}(T)=\eta_{T}^{*}$. In this case however, the initial boundary value $\eta_{0}^{*}$ is reached asymptotically for $t \rightarrow-\infty$ leading to the pre-actuation interval $t \in(-\infty, 0]$ for the feedforward control (2.34), also see Figure 1.3.

The transition time $T$ can be used as free parameter in the feedforward control design, if the stationary boundary values $\boldsymbol{\eta}_{0}^{*}$ and $\boldsymbol{\eta}_{T}^{*}$ in (2.32) are not connected by a quasi-stationary connection as in case of the double pendulum swing-up in Section 2.4. Then, depending on the setup function $\Upsilon(t, \boldsymbol{p})$, certain regions of the transition time $T$ exist for the solution of the $B V P$ (2.32) (see Remark 2.4). Alternatively, $T$ can be determined in dependence of a desired aggressiveness of the feedforward control $u^{*}(t)$ with respect to input constraints, see Section 3.2. 


\subsection{Numerical solution of the BVP with free parameters}

The solution of two-point BVPs with free parameters as given in (2.32) for the state $\boldsymbol{\eta}^{*}(t)$ and the parameter set $\boldsymbol{p}$ is a standard task in numerics. Various methods exist for their solution like shooting, finite difference schemes, and collocation, see e.g. (Keller, 1968; Ascher et al., 1988) as well as Appendix B for a short introduction. Directly linked to the numerical solution of the BVP is the question concerning existence and uniqueness of the solution. A discussion of these points is given in the following. Thereafter, the standard MATLAB function bvp4c is shortly introduced, which provides a convenient and efficient way to solve two-point BVPs.

\subsubsection{Discussion on solvability of the BVP}

The key role in the feedforward control design plays the numerical solution of the BVP (2.32) of the internal dynamics. A difficult question concerns the existence and uniqueness of a solution for nonlinear two-point BVPs like (2.32). Explicit results are only available for linear systems and certain nonlinear second-- and third-order systems, see e.g. (Bailey et al., 1968). For general nonlinear two-point BVPs as given in (2.32), only few conservative existence and uniqueness theorems exist based on analytical considerations, see e.g. (Keller, 1968, Theorem 1.2.6). From the numerical point of view, further conditions can be found in the literature where the existence of a solution of a nonlinear two-point BVP is linked to its numerical solvability. For instance, if finite difference methods are used for the BVP solution, contraction mapping theorems can be applied to analyze the numerical solvability of the finite difference equations (Dennis and Schnabel, 1983; Ascher et al., 1988; Taylor and Li, 2002). However, these theorems for the solvability of nonlinear two-point BVPs based on analytical and numerical viewpoints are hardly practically applicable.

On the other hand, the solvability of the internal dynamics BVP (2.32) implies the question if the feedforward control (2.26) exists for the finite-time transition problem. From this viewpoint, the solution of the BVP (2.32) can be seen as a constructive controllability analysis of the considered system. Hence, the difficult issue concerning solvability of the BVP (2.32) corresponds in some sense to the nontrivial investigation of controllability of nonlinear systems.

A "heuristical" controllability criterion can be stated if the considered setpoints of the transition problem are linked by a quasi-stationary curve via a connected set of equilibria. Then, for a sufficiently large transition time $T$, the state trajectories will approach the stationary curve which implies existence of a solution for the internal dynamics BVP (2.32).

\subsubsection{Matlab function bvp4c}

The Matlab function bvp4c (Shampine et al., 2000; Kierzenka and Shampine, 2001; Shampine et al., 2003) solves two-point BVPs of the form

$$
\dot{\boldsymbol{\zeta}}=\boldsymbol{f}(\boldsymbol{\zeta}, t, \boldsymbol{p}), \quad t \in(0, T), \quad \boldsymbol{g}(\boldsymbol{\zeta}(0), \boldsymbol{\zeta}(T), \boldsymbol{p})=\mathbf{0}
$$


for the state $\boldsymbol{\zeta}(t)$ on a finite interval $t \in[0, T]$. The function bvp4c also determines unknown parameters $\boldsymbol{p}$, which directly corresponds to the set of free parameters $\boldsymbol{p}$ in the BVP (2.32). Thereby, the condition

$$
\operatorname{dim} \boldsymbol{p}=\operatorname{dim} \boldsymbol{g}-\operatorname{dim} \boldsymbol{\zeta}
$$

concerning the number of free parameters and BCs has to be satisfied. The function bvp4c employs the collocation method described in Appendix B.1. By dividing the interval $[0, T]$ into subintervals, bvp4c determines the solution $\boldsymbol{\zeta}(t)$ and $\boldsymbol{p}$ by solving a set of nonlinear algebraic equations resulting from the collocation conditions imposed on the subintervals and the BCs in (2.37). Thereby, the numerical solution is obtained with fourth-order accuracy. A particular feature of bvp4c is the adaptation of the mesh discretization in order to further increase the accuracy of the numerical solution.

The MatLaB function bvp4c can be used in a straightforward manner to solve the BVP (2.32) if the ODEs, the time interval, and the BCs are appropriately adapted to the form of (2.37). Thereby, the linear BCs in (2.32) must be formulated in the residual representation

$$
\boldsymbol{\eta}^{*}(0)-\boldsymbol{\eta}_{0}^{*}=\mathbf{0}, \quad \boldsymbol{\eta}^{*}(T)-\boldsymbol{\eta}_{T}^{*}=\mathbf{0}
$$

Since the nonlinear algebraic equations stemming from the collocation are solved by a Newton iteration, bvp4c requires a suitable guess of the trajectories $\boldsymbol{\eta}^{*}\left(t_{k}\right)=\left[\eta_{1}^{*}\left(t_{k}\right), \ldots, \eta_{n-r}^{*}\left(t_{k}\right)\right]^{\top}$ on an initial time mesh $t_{k} \in[0, T], k=1, \ldots, N$ as well as initial values of the unknown parameter set $\boldsymbol{p}$. For most applications, reasonable guesses for $\eta_{i}^{*}\left(t_{k}\right)$ are linear interpolations between the BCs $\eta_{i}^{*}(0)=\eta_{0, i}^{*}$ and $\eta_{i}^{*}(T)=\eta_{T, i}^{*}, i=1, \ldots, n-r$ on a uniform mesh grid $t_{k} \in[0, T], k=1, \ldots, N$ with e.g. $N=30$ mesh points. An obvious guess for the unknown parameter set is $\boldsymbol{p}=\mathbf{0}$ corresponding to a monotonic output trajectory $y^{*}(t)=\Upsilon(t, \mathbf{0})$ between the setpoints $y_{0}^{*}$ and $y_{T}^{*}$, as schematically shown in Figure 2.1. Moreover, the robustness and convergence of the numerical solution can by enhanced by providing the analytical Jacobians

$$
\boldsymbol{J}_{\zeta}=\frac{\partial \boldsymbol{f}(\boldsymbol{\zeta}, t, \boldsymbol{p})}{\partial \boldsymbol{\zeta}}, \quad \boldsymbol{J}_{\boldsymbol{p}}=\frac{\partial \boldsymbol{f}(\boldsymbol{\zeta}, t, \boldsymbol{p})}{\partial \boldsymbol{p}}
$$

to the function bvp4c.

Due to the algebraic solution technique of the BVP solver bvp4c, there is no distinction whether the considered internal dynamics (2.32) is stable or unstable, since its solution is obtained without numerical time integration in contrast to shooting techniques. Therefore, the feedforward control design is applicable in the same manner for linear and nonlinear systems with stable and unstable internal dynamics, i.e. for both minimum-phase and nonminimum-phase systems. ${ }^{7}$

Example 2.4 (Example 2.3 continued). The internal dynamics BVP (2.35) of the linear SISO system (1.1) together with the setup function $\Upsilon(t, p)$ in (2.36) are implemented under bvp4c. A linear interpolation between the corresponding BCs in (2.35) on a uniform mesh

\footnotetext{
${ }^{7}$ An alternative to bvp4c is e.g. the MATLAB solver sbvp (Auzinger et al., 2003a) for BVPs with singularities or general collocation or finite-difference schemes, see Appendix B.
} 
$t_{k} \in[0, T], k=1, \ldots, 30$ is used as reasonable guess for the trajectory $\eta^{*}\left(t_{k}\right)$. The initial guess of the free parameter is $p=0$. The function bvp4c returns the trajectory $\eta^{*}(t), t \in[0, T]$ and the free parameter $p$. Finally, the output trajectory $y^{*}(t)$ and the feedforward control $u^{*}(t)$ follow from (2.36) and (2.34). Figure 2.4 shows the nominal trajectories $\eta^{*}(t), y^{*}(t)$, and $u^{*}(t)$ for the setpoint transition $y_{0}^{*}=0 \rightarrow y_{T}^{*}=1$ corresponding to the flatness-based feedforward design in Figure 2.2. The boundary values in (2.35) follow from (2.25) and (2.15) to $\eta_{0}^{*}=0$ and $\eta_{T}^{*}=2.25$. The free parameter values for the three transition times $T \in\{1,1.5,2\}$ are $p \in\{-30.2,-20.3,-15.4\}$ and determine the undershoot in the output trajectory (2.36), which is characteristic for nonminimum-phase systems. Moreover, the undershoot increases for smaller transition times $T$.
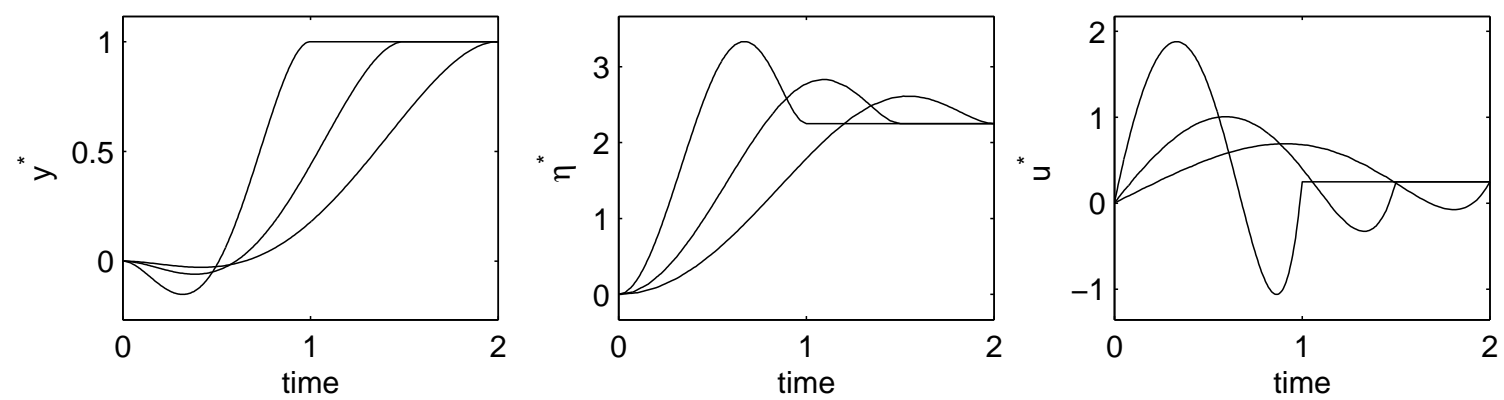

Figure 2.4: Setpoint transition $y_{0}^{*}=0 \rightarrow y_{T}^{*}=1$ and $u_{0}^{*}=0 \rightarrow u_{T}^{*}=1$ for the linear SISO system (1.1) based on the solution of the BVP (2.35) with the parameters (2.15) and various transition times $T \in\{1,1.5,2\}$.

\subsection{Example - Swing-up of the double pendulum on a cart}

Pendulums are widely used in nonlinear control education and research as benchmark examples of underactuated mechanical systems. A vast range of contributions exists for the stabilization of different types of inverted pendulums, see e.g. (Mori et al., 1976; Furuta et al., 1980; Anderson and Grantham, 1989; Gros et al., 2006). Besides the stabilization aspect, the swing-up problem - especially of the classic single pendulum on a cart - has gained increasing attention during the recent past, see e.g. (Wiklund et al., 1993; Åström and Furuta, 2000). Furthermore, the swing-up of various types of double pendulums is addressed in the literature, like the acrobot and pendubot (Spong, 1995; Fantoni et al., 2000; Graichen and Zeitz, 2004, 2005a), or the rotary double pendulum in (Yamakati et al., 1993, 1995).

A particularly challenging problem is the swing-up of the double pendulum on a cart, which is less accounted for in the literature. This is mainly due to the limited rail length of the cart, in contrast e.g. to the rotary double pendulum. In (Zhong and Röck, 2001; Huang and Fu, 2003), a passivity-based approach is proposed in combination with partial feedback linearization. The 
swing-up of the double pendulum on a cart is accomplished in simulation studies, but no experimental results are provided in (Zhong and Röck, 2001; Huang and Fu, 2003).

Another approach (Rubí et al., 2002) utilizes the two-degree-of-freedom control scheme in Figure 1.1 to solve the swing-up problem. The feedforward control as well the nominal state trajectories for the swing-up are obtained by solving an optimization problem with the stationary downward and upward equilibria as boundary conditions. The underactuated dynamics of the double pendulum is taken into account by considering all links of the double pendulum to be active and minimizing the torques exerted at the unactuated links. However, the obtained trajectory is only an approximate solution for the swing-up problem since the torques acting at the free joints are not identically zero. A gain-scheduled feedback control is used to stabilize the system during the swing-up and in the upward position. To the author's knowledge, (Rubí et al., 2002) is the only contribution so far providing experimental results for the swing-up of the double pendulum on a cart.

In the following, the inversion-based feedforward control design is applied to the swing-up problem of the double pendulum on a cart (Graichen et al., 2007). The feedforward design is based on the nonlinear equations of motion which are derived by the Lagrangian method. The model parameters correspond to the experimental construction of the pendulum which is used to validate the swing-up maneuver. Due to the accuracy of the nonlinear feedforward control, a linear feedback controller based on an LQ design is sufficient to stabilize the pendulum along the nominal trajectories.

\subsubsection{Problem statement}

The double pendulum on a cart (see Figure 2.5) consists of two links with the length $l_{i}$ and the angles $\eta_{i}, i=1,2$ to the vertical. By choosing the displacement of the cart as output $y$, the pendulum is directly described in the input-output coordinates (2.17). The mechanical parameters are described in Table 2.1 together with their corresponding values, which have been measured and identified at the experimental device (see Section 2.4.3). Furthermore, the cart is subject to the constraints

$$
|y| \leq 0.7 \mathrm{~m}, \quad|\dot{y}| \leq 2.5 \mathrm{~m} / \mathrm{s}, \quad|\ddot{y}| \leq 20 \mathrm{~m} / \mathrm{s}^{2}
$$

due to the limited rail length and the physical limitations of the cart actuator.

\subsubsection{Equations of motion}

The mathematical model of the double pendulum can be derived via the Lagrangian method. The absolute position $\boldsymbol{x}^{i}=\left[x_{1}^{i}, x_{2}^{i}\right]^{\top}, i=1,2$ of the center of mass of each link $i$ is given by

$$
\text { Link 1: } \quad \boldsymbol{x}^{1}=\left[\begin{array}{c}
y-a_{1} \sin \eta_{1} \\
a_{1} \cos \eta_{1}
\end{array}\right], \quad \text { Link 2: } \quad \boldsymbol{x}^{2}=\left[\begin{array}{c}
y-l_{1} \sin \eta_{1}-a_{2} \sin \eta_{2} \\
l_{1} \cos \eta_{1}+a_{2} \cos \eta_{2}
\end{array}\right] .
$$




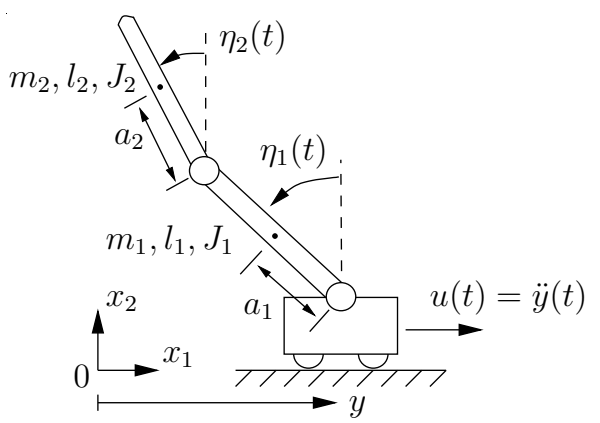

Figure 2.5: Schematic of the double pendulum with the mechanical parameters in Table 2.1 .

\begin{tabular}{lcc}
\hline Pendulum link & $\begin{array}{c}\text { inner } \\
i=1\end{array}$ & $\begin{array}{l}\text { outer } \\
i=2\end{array}$ \\
\hline length $l_{i}[\mathrm{~m}]$ & 0.323 & 0.480 \\
distance to center of gravity $a_{i}[\mathrm{~m}]$ & 0.215 & 0.223 \\
mass $m_{i}[\mathrm{~kg}]$ & 0.853 & 0.510 \\
moment of inertia $J_{i}\left[\mathrm{~N} \mathrm{~m} \mathrm{~s}^{2}\right]$ & 0.013 & 0.019 \\
friction constant $d_{i}[\mathrm{~N} \mathrm{~ms}]$ & 0.005 & 0.005 \\
\hline
\end{tabular}

Table 2.1: Mechanical parameters of the double pendulum.

The kinetic and potential energies are determined to

$$
T=\frac{1}{2} m_{c} \dot{y}^{2}+\frac{1}{2} \sum_{i=1}^{2}\left[m_{i}\left|\dot{\boldsymbol{x}}^{i}\right|^{2}+J_{i} \dot{\eta}_{i}^{2}\right], \quad V=\sum_{i=1}^{2} m_{i} g x_{2}^{i} .
$$

The Lagrangian $L=T-V$ yields the equations of motion

$$
\frac{\mathrm{d}}{\mathrm{d} t} \frac{\partial L}{\partial \dot{\eta}_{i}}-\frac{\partial L}{\partial \eta_{i}}=Q_{i}, \quad i=1,2
$$

The generalized non-conservative friction forces $Q_{i}$ with respect to the generalized coordinates $\eta_{1}$ and $\eta_{2}$ are modeled with linear expressions:

$$
Q_{1}=-d_{1} \dot{\eta}_{1}+d_{2}\left(\dot{\eta}_{2}-\dot{\eta}_{1}\right), \quad Q_{2}=d_{2}\left(\dot{\eta}_{1}-\dot{\eta}_{2}\right)
$$

whereby the parameters $d_{i}$ denote the damping coefficient at the respective link $i$. The equations of motion (2.42) can be written in the matrix notation

$$
G \ddot{\boldsymbol{\eta}}+\boldsymbol{g}=\mathbf{0}
$$

with the matrix $G$ and the vector $\boldsymbol{g}$ depending on $\boldsymbol{\eta}=\left[\eta_{1}, \eta_{2}\right]^{\top}, \dot{\boldsymbol{\eta}}$, and $\ddot{y}$. The matrix $G$ and the elements of $\boldsymbol{g}=\left[g_{1}, g_{2}\right]^{\top}$ are given in Table 2.2.

In the double pendulum experiment (see Section 2.4.3), the acceleration $\ddot{y}$ of the cart serves as input $u=\ddot{y}$ to the system, such that the overall model of the double pendulum can be formally written in the input-output normal form (2.18)-(2.19)

$$
\begin{aligned}
& \ddot{y}=u \\
& \ddot{\boldsymbol{\eta}}=-G^{-1} \boldsymbol{g}=\boldsymbol{\beta}(\boldsymbol{\eta}, \dot{\boldsymbol{\eta}}, u)
\end{aligned}
$$

with relative degree $r=2, \boldsymbol{\beta}=\left[\beta_{1}, \beta_{2}\right]^{\top}$, and the system order $n=6$. The internal dynamics (2.45) is given as second-order ODE, as it is commonly done for mechanical systems. 


$$
\begin{aligned}
G & =\left[\begin{array}{cc}
J_{1}+a_{1}^{2} m_{1}+l_{1}^{2} m_{2} & a_{2} l_{1} m_{2} \cos \left(\eta_{1}-\eta_{2}\right) \\
a_{2} l_{1} m_{2} \cos \left(\eta_{1}-\eta_{2}\right) & J_{2}+a_{2}^{2} m_{2}
\end{array}\right] \\
g_{1} & =d_{1} \dot{\eta}_{1}+d_{2}\left(\dot{\eta}_{1}-\dot{\eta}_{2}\right)+l_{1} m_{2} a_{2} \sin \left(\eta_{1}-\eta_{2}\right) \dot{\eta}_{2}^{2}-\left(a_{1} m_{1}+l_{1} m_{2}\right)\left[g \sin \left(\eta_{1}\right)+\cos \left(\eta_{2}\right) \ddot{y}\right] \\
g_{2} & =d_{2}\left(\eta_{2}-\eta_{1}\right)-a_{2} m_{2}\left[g \sin \left(\eta_{2}\right)+l_{1} \sin \left(\eta_{1}-\eta_{2}\right) \dot{\eta}_{1}^{2}+\cos \left(\eta_{2}\right) \ddot{y}\right]
\end{aligned}
$$

Table 2.2: Matrix $G$ and vector $\boldsymbol{g}=\left[g_{1}, g_{2}\right]^{\top}$ for the equations of motion (2.43) of the double pendulum in Figure 2.5.

\subsubsection{Swing-up problem}

The swing-up within a finite time interval $t \in[0, T]$ requires to steer the double pendulum from the initial downward equilibrium

$$
y(0)=0, \quad \dot{y}(0)=0, \quad \boldsymbol{\eta}(0)=[-\pi,-\pi]^{\top}=-\boldsymbol{\pi}, \quad \dot{\boldsymbol{\eta}}(0)=\mathbf{0}
$$

to the terminal upward equilibrium

$$
y(T)=0, \quad \dot{y}(T)=0, \quad \boldsymbol{\eta}(T)=\mathbf{0}, \quad \dot{\boldsymbol{\eta}}(T)=\mathbf{0} .
$$

The downward equilibrium is weakly asymptotically stable due to the friction in the joints, whereas the upward position is unstable. The ODEs (2.44)-(2.45) together with the BCs (2.46)-(2.47) form a nonlinear two-point boundary value problem (BVP) for the states $y(t)$, $\dot{y}(t)$, and $\boldsymbol{\eta}(t), \dot{\boldsymbol{\eta}}(t)$ in dependence of the input trajectory $u(t)$, which is the main objective of the feedforward control design.

The swing-up time $T$ is an important design parameter and mainly depends on the system dynamics (2.44)-(2.45) and the constraints (2.41). If $T$ is chosen too small, the cart may violate the constraints. On the other hand, the swing-up is not possible arbitrarily slowly due to the fact that no quasi-stationary connection exists between the downward and upward equilibria, i.e. they are not connected by a set of equilibria in between. ${ }^{8}$ Hence, the determination of an appropriate swing-up time $T$ is one of the main issues of the feedforward control design.

\subsubsection{Feedforward control design}

The feedforward control is obtained by inverting the input-output dynamics (2.18). In view of (2.44), the feedforward control

$$
u^{*}(t)=\ddot{y}^{*}(t)
$$

is simply the second time derivative of the desired output trajectory $y^{*}(t)$. Thereby, $y^{*}(t)$ has to satisfy the four BCs in (2.46)-(2.47) for the output which implies $y^{*}(t) \in \mathcal{C}^{1}$, i.e. the

\footnotetext{
${ }^{8}$ The problem concerning the non-existence of a quasi-stationary connection between the equilibria is addressed in more detail in (Graichen and Zeitz, 2005a; Bermes, 2005), where the swing-up problem of the pendubot - a polar double pendulum - is considered.
} 
output trajectory must be at least once differentiable. For the experimental setup of the double pendulum described in Section 2.4.3, it is indeed sufficient to plan a $\mathcal{C}^{1}$-continuous output trajectory $y^{*}(t)$.

\subsubsection{Boundary value problem of the internal dynamics}

In order to determine the trajectories $\boldsymbol{\eta}^{*}(t)$ and $\dot{\boldsymbol{\eta}}^{*}(t)$ of the angles, the internal dynamics (2.45) can be rewritten by inserting the feedforward control (2.48), i.e.

$$
\ddot{\boldsymbol{\eta}}^{*}=\boldsymbol{\beta}\left(\boldsymbol{\eta}^{*}, \dot{\boldsymbol{\eta}}^{*}, \ddot{y}^{*}\right), \quad \boldsymbol{\eta}^{*}(0)=-\boldsymbol{\pi}, \quad \boldsymbol{\eta}^{*}(T)=\mathbf{0},\left.\quad \dot{\boldsymbol{\eta}}^{*}\right|_{t=0, T}=\mathbf{0}
$$

subject to the respective BCs in (2.46)-(2.47). Thereby, the zero dynamics (for $\ddot{y}^{*}=0$ ) is stable in the downward position but is unstable in the upward position, i.e. the inverted double pendulum is nonminimum-phase.

Since the BVP (2.49) for the swing-up problem is overdetermined by $2(n-r)=8$ BCs for $n-r=4$ ODEs, the output trajectory (2.29) requires $n-r=4$ free parameters $\boldsymbol{p}=\left(p_{1}, \ldots, p_{4}\right)$ in a $\mathcal{C}^{1}$-continuous setup function $\Upsilon(t, \boldsymbol{p})$, which is constructed using the cosine series $(2.31 \mathrm{~b})$ :

$$
y^{*}(t)=\Upsilon(t, \boldsymbol{p})=a_{0}+a_{1} \cos \left(\frac{\pi t}{T}\right)+\sum_{i=1}^{4} p_{i} \cos \left(\frac{(1+i) \pi t}{T}\right) .
$$

The choice of the coefficients $a_{0}=-p_{1}-p_{3}$ and $a_{1}=-p_{2}-p_{4}$ (see Table A.2) ensures that $\Upsilon(0, \boldsymbol{p})=\Upsilon(T, \boldsymbol{p})=0$ is satisfied independent of the parameter values $\boldsymbol{p}$. Moreover, the BCs $\dot{\Upsilon}(0, \boldsymbol{p})=\dot{\Upsilon}(T, \boldsymbol{p})=0$ are directly fulfilled by the sine terms occurring in the first time derivative of $(2.50)$.

Remark 2.4. The swing-up time $T$ is an important design parameter of the swing-up problem and is directly affected by the choice of the setup function $y^{*}(t)=\Upsilon(t, \boldsymbol{p})$. For instance, the number of times that the output $y^{*}(t)$ passes through zero ("swinging" of the cart) is limited by the highest frequency of $\Upsilon(t, \boldsymbol{p})$ in (2.50). This corresponds to certain regions of $T$ where solutions for the swing-up problem exist. Alternatively, the swing-up time can be treated as a free parameter (via time transformation) with the remaining three parameters $\boldsymbol{p}=\left(p_{1}, p_{2}, p_{3}\right)$ provided in the setup function $\Upsilon(t, \boldsymbol{p})$, see (Graichen and Zeitz, 2005a; Treuer, 2005).

\subsubsection{Numerical results}

The internal dynamics BVP (2.49) with the output trajectory $y^{*}(t)=\Upsilon(t, \boldsymbol{p})$ in $(2.50)$ is solved with the MATLAB function bvp4c. The initial guess for the trajectories $\boldsymbol{\eta}^{*}\left(t_{k}\right)$ and $\dot{\boldsymbol{\eta}}^{*}\left(t_{k}\right)$ is a linear interpolation between the BCs in (2.49) on a uniform time mesh with 30 points $t_{k} \in[0, T], k=1, \ldots, 30$. The initial guess of the free parameters is $p_{i}=0, i=1, \ldots, 4$. bvp4c returns the trajectories $\boldsymbol{\eta}^{*}(t)=\left[\eta_{1}^{*}(t), \eta_{2}^{*}(t)\right]^{\top}$ and the parameter set $\boldsymbol{p}$, which yields the output trajectory $y^{*}(t)=\Upsilon(t, \boldsymbol{p})$ and the feedforward control $(2.48)$, i.e. $u^{*}(t)=\ddot{\Upsilon}(t, \boldsymbol{p})$. 

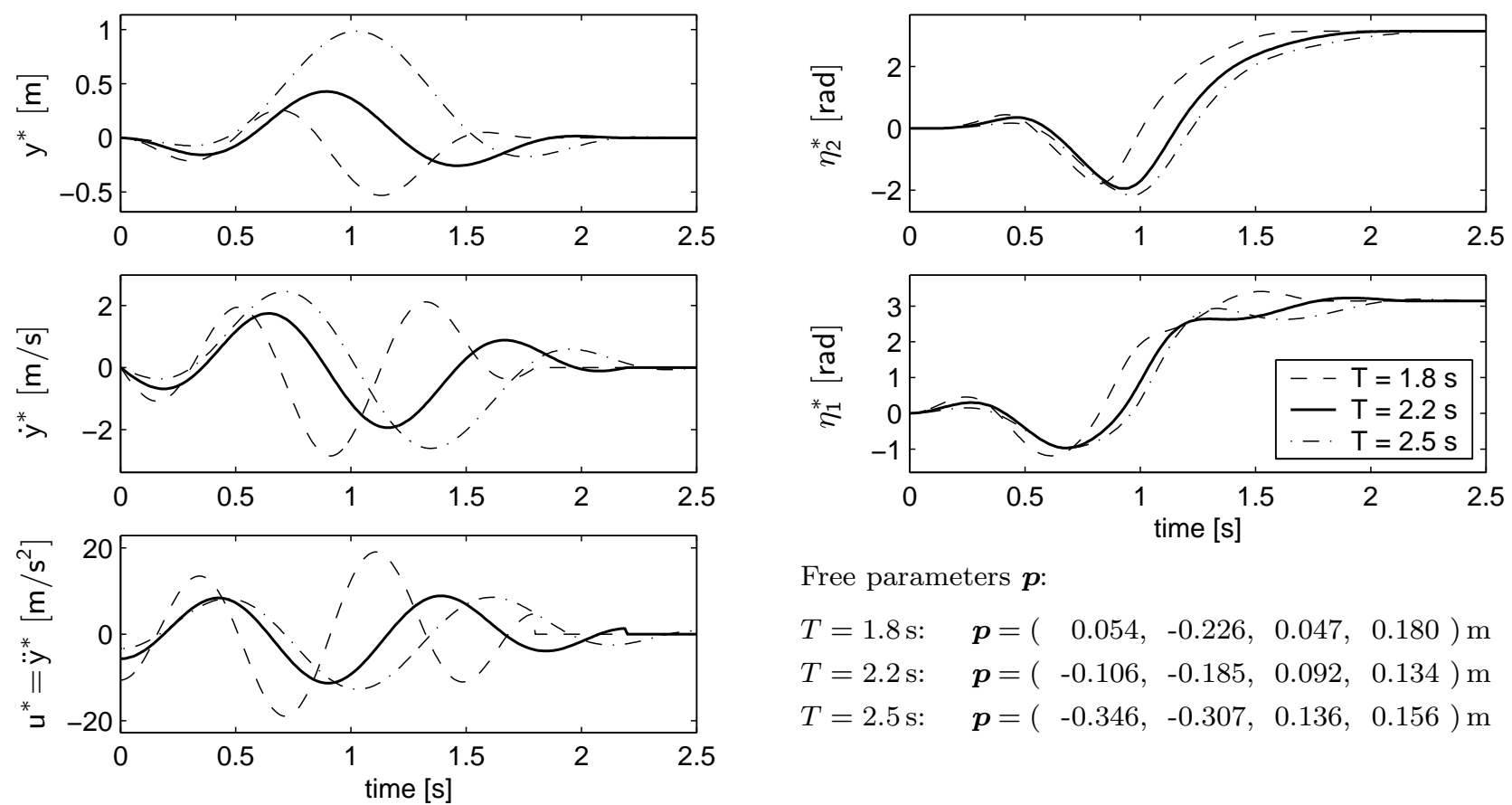

Free parameters $\boldsymbol{p}$ :

$T=1.8 \mathrm{~s}: \quad \boldsymbol{p}=\left(\begin{array}{llll}0.054, & -0.226, & 0.047, & 0.180\end{array}\right) \mathrm{m}$

$T=2.2 \mathrm{~s}: \quad \boldsymbol{p}=\left(\begin{array}{llll}-0.106, & -0.185, & 0.092, & 0.134\end{array}\right) \mathrm{m}$

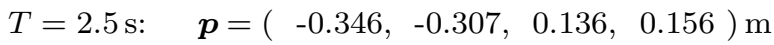

Figure 2.6: Nominal trajectories and parameters $\boldsymbol{p}$ for the open-loop swing-up of the double pendulum with three different swing-up times $T$.

Figure 2.6 shows the nominal trajectories for the swing-up maneuver for different swing-up times $T$ in the interval $1.8 \mathrm{~s} \leq T \leq 2.5 \mathrm{~s}$ where reasonable solutions of the BVP (2.49) could be found (see Remark 2.4). The significant influence of the swing-up time $T$ is particularly apparent in the cart trajectory $y^{*}(t)$, its velocity $\dot{y}^{*}(t)$, and acceleration $\ddot{y}^{*}(t)$. For $T=1.8 \mathrm{~s}$, the cart movement $y^{*}(t)$ is very limited, but the required maximum acceleration $\max _{t} \ddot{y}^{*}(t)=$ $19 \mathrm{~m} / \mathrm{s}^{2}$ almost hits the respective constraint in (2.41). The swing-up time $T=2.5 \mathrm{~s}$ leads to a different swing-up motion with the large cart displacement $\max _{t} y^{*}(t)=1 \mathrm{~m}$. A good trade-off between the maximum amplitudes of the trajectories $y^{*}(t), \dot{y}^{*}(t), \ddot{y}^{*}(t)$ with respect to the constraints (2.41) is obtained for the swing-up time $T=2.2 \mathrm{~s}$, which is therefore chosen as swing-up time for the experimental validation.

Figure 2.7 shows time-discrete snapshots of the pendulum for the swing-up time $T=2.2 \mathrm{~s}$ to illustrate its motion. It is interesting to mention that both arms of the pendulum are in a hinged position during the swing-up (see sequences 2 and 3 in Figure 2.7) and only stretch close to the upward "inverted" position.

Remark 2.5. An alternative to using the cosine series (2.31b) for the setup of the output trajectory $y^{*}(t)=\Upsilon(t, \boldsymbol{p})$ is the polynomial (2.31a). However, the iteratively determined time interval $2.0 \mathrm{~s} \leq T \leq 2.2 \mathrm{~s}$ for reasonable solutions of the $B V P(2.49)$ is considerably smaller. Moreover, the steps in the acceleration $\ddot{y}^{*}(t)=\ddot{\Upsilon}(t, \boldsymbol{p})$ at the swing-up bounds $t=0$ and $t=T$ are larger than in case of the cosine setup (2.50). 

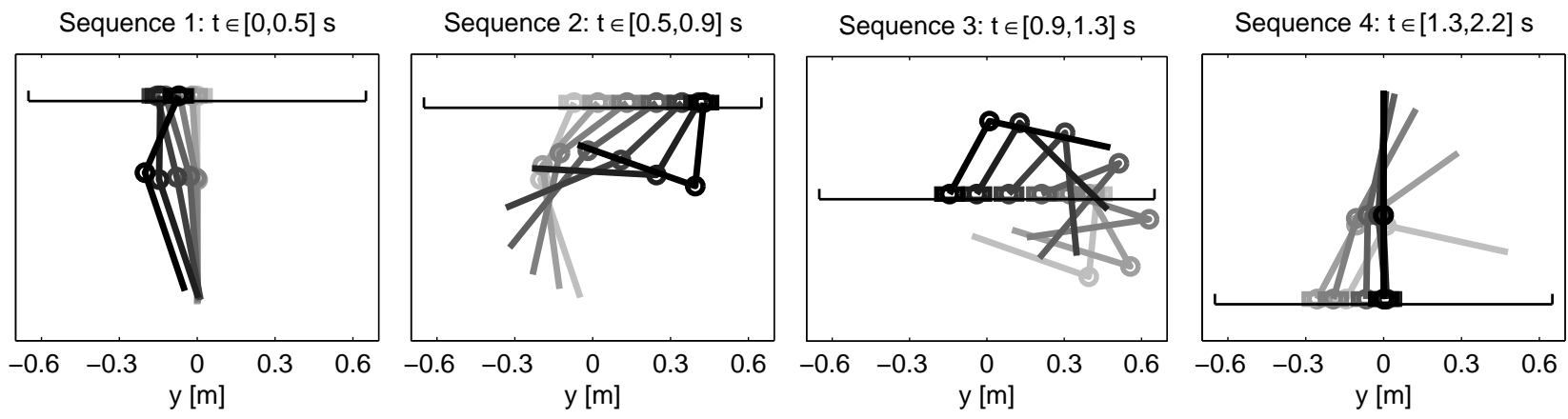

Figure 2.7: Snapshots of the nominal swing-up for $T=2.2 \mathrm{~s}$ depicted in four sequences with increasing darkness of the snapshots as time increases during the respective sequence.

\subsubsection{Experimental validation}

The experimental realization of the swing-up maneuver requires a stabilization of the double pendulum by a feedback controller. In the context of the two-degree-of-freedom control scheme in Figure 2.8, the feedforward control $\Sigma_{F F}$ is supported by a state feedback control $\Sigma_{F B}$ with an observer $\widehat{\Sigma}$ in order to stabilize the system $\Sigma$ along the nominal trajectories $\boldsymbol{x}^{*}(t)$ provided by the signal generator $\Sigma^{*}$. Thereby, a highly accurate feedforward control $\Sigma_{F F}$ is necessary in order to minimize the demands on the feedback part during the swing-up. The accuracy of the nonlinear feedforward control can be enhanced by an optimization-based adjustment of the mechanical parameters in Table 2.1 with respect to the open-loop experimental results for the nominal feedforward control $u^{*}(t)$ (Graichen et al., 2007). The experimental setup and the above-mentioned points are addressed in the following subsections.

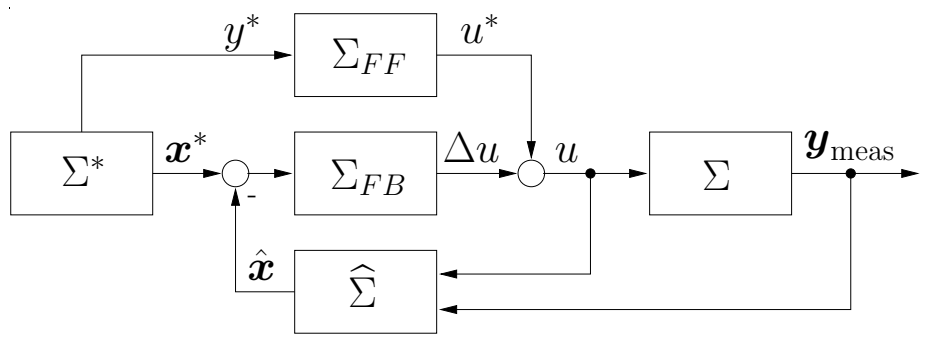

Figure 2.8: Two-degree-of-freedom control scheme with system $\Sigma$, signal generator $\Sigma^{*}$, feedforward control $\Sigma_{F F}$, feedback control $\Sigma_{F B}$, observer $\widehat{\Sigma}$, and measurement vector $\boldsymbol{y}_{\text {meas }}=\left[y, \phi_{1}, \phi_{2}\right]^{\top}$.

\subsubsection{Experimental setup of the double pendulum}

The swing-up maneuver is experimentally realized with the double pendulum in Figure 2.9 corresponding to the model parameters in Table 2.1 and the cart constraints (2.41). ${ }^{9}$ The

\footnotetext{
${ }^{9}$ The construction of the pendulum was a joint project of the Max Planck Institute for Dynamics of Complex Technical Systems (www.mpi-magdeburg.mpg.de) and the company Hasomed GmbH (www.hasomed.de). The pendulum can also be employed with three links, see Section 4.4 and e.g. (Graichen et al., 2005c) for the side-stepping of the triple inverted pendulum.
} 
incremental angle encoders at the two joints have a resolution of $2 \pi / 8192 \mathrm{rad}$ and transmit their information through optical links in the joints to reduce friction. The cart is driven by a toothed belt connected to a synchronous motor. The control algorithm is implemented on a $933 \mathrm{MHz}$ computer with Real-Time Linux and the sampling time $1 \mathrm{~ms}$. The nominal trajectories $\boldsymbol{\eta}^{*}(t), \dot{\boldsymbol{\eta}}^{*}(t), y^{*}(t), \dot{y}^{*}(t)$, and the feedforward control $u^{*}(t)=\ddot{y}^{*}(t), t \in[0, T]$ are calculated offline and stored in look-up tables.

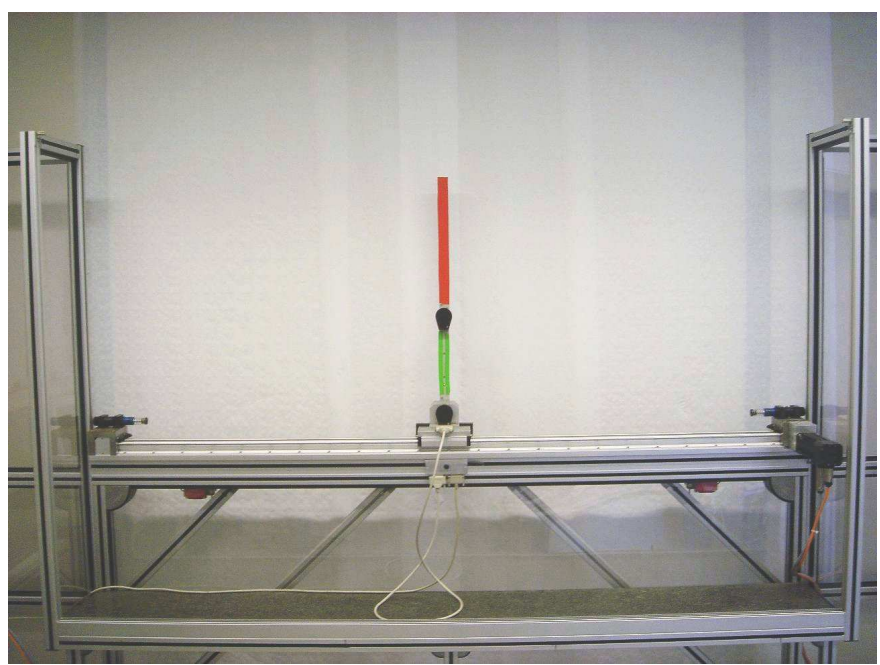

Figure 2.9: Experimental construction of the double pendulum on a cart (Hasomed GmbH) with model parameters in Table 2.1 and the cart constraints (2.41).

Remark 2.6. The experimental device in Figure 2.9 uses an underlying fast PI control for the velocity $\dot{y}$ of the cart instead of controlling the acceleration $u=\ddot{y}$ in the pendulum model (2.44)-(2.45). Therefore, $u$ is integrated before it is used as setpoint for the cascaded velocity controller. This justifies the use of the output trajectory (2.50) leading to discontinuities of the feedforward control $u^{*}(t)$ at the time instants $t=0$ and $t=T$. Due to the internal integration of $u=\ddot{y}$, a step at the time instants $t=0$ and $t=T$ results in a continuous velocity $\dot{y}$ which the cascaded PI controller is able to follow.

\subsubsection{Optimization-based adjustment of model parameters}

The feedforward control $u^{*}(t)$ must be highly accurate in order to steer the double pendulum along the nominal swing-up trajectories close to the unstable upward equilibrium. If the feedforward control $u^{*}(t)$ is too inaccurate, the pendulum drifts away from the nominal trajectories too early, and the feedback control has to correct the tracking error. As a result, the deviations of the cart position $y(t)$ from its nominal trajectory $y^{*}(t)$ might exceed the maximum rail length (2.41). In order to enhance the accuracy of the feedforward control $u^{*}(t)$, the mechanical parameters (Table 2.1) of the pendulum model (2.44)-(2.45) are adjusted by solving an optimization problem with respect to the experimental swing-up maneuver in open-loop. 
Figure 2.10a shows the measured trajectories (-) of the angles $\eta_{1}(t)$ and $\eta_{2}(t)$ for the swing-up maneuver compared with the nominal open-loop trajectories (- -$)$ in Figure 2.6 for $T=2.2 \mathrm{~s}$ and the default parameters in Table 2.1. The angle $\eta_{1}(t)$ of the inner arm stays close to the nominal trajectory $\eta_{1}^{*}(t)$ almost up to the unstable upward equilibrium, but the outer arm angle $\eta_{2}(t)$ follows $\eta_{2}^{*}(t)$ only at the beginning of the swing-up. For instance, at the time $t=1.3 \mathrm{~s}$, $\eta_{2}(t)$ is $0.9 \mathrm{rad} \approx 50 \mathrm{deg}$ away from $\eta_{2}^{*}(t)$. The inaccuracy is mainly caused by the time-delayed response of the underlying PI controller for the cart velocity (see Remark 2.6) as well as due to unmodeled effects like nonlinear friction and uncertain model parameters.

In order to meet the measured angle profiles $\eta_{1}(t), \eta_{2}(t)$ in Figure 2.10a as close as possible, the parameters $\boldsymbol{\theta}=\left(a_{1}, a_{2}, m_{1}, m_{2}, J_{1}, J_{2}, d_{1}, d_{2}\right)$ occurring in the pendulum dynamics (2.45) (also see Table 2.2) are adjusted by solving the optimization problem

$$
\begin{aligned}
\min _{\theta} I & =\int_{0}^{T_{0}}\left(\eta_{\theta, 1}(t)-\eta_{1}(t)\right)^{2}+\left(\eta_{\theta, 2}(t)-\eta_{2}(t)\right)^{2} \mathrm{~d} t \\
\text { subject to } \quad \ddot{\boldsymbol{\eta}}_{\theta} & =\boldsymbol{\beta}\left(\boldsymbol{\eta}_{\theta}, \dot{\boldsymbol{\eta}}_{\theta}, u^{*}\right), \quad \boldsymbol{\eta}_{\theta}(0)=-\boldsymbol{\pi}, \quad \dot{\boldsymbol{\eta}}_{\theta}(0)=\mathbf{0} .
\end{aligned}
$$

The feedforward control $u^{*}(t)$ is based on the default parameter values $\boldsymbol{\theta}_{\text {nom }}$ (see Table 2.1 and Figure 2.6) and serves as input to the pendulum dynamics (2.52) with the states $\boldsymbol{\eta}_{\theta}=\left[\eta_{\theta, 1}, \eta_{\theta, 2}\right]^{\top}$ and $\dot{\boldsymbol{\eta}}_{\theta}$. The cost function $I$ in (2.51) rates the deviation of the angles $\eta_{\theta, 1}(t), \eta_{\theta, 2}(t)$ with respect to the measured open-loop trajectories $\eta_{1}(t), \eta_{2}(t)$ in Figure 2.10a. Thereby, $I$ is evaluated over the time interval $t \in\left[0, T_{0}\right]$, whereby $T_{0}=1.6 \mathrm{~s}$ is chosen smaller than the swing-up time $T=2.2 \mathrm{~s}$, because the dynamics (2.52) turns unstable at the end of the swing-up, leading to an unstable numerical integration close to the upward equilibrium. It is important to mention that

(a): default parameters
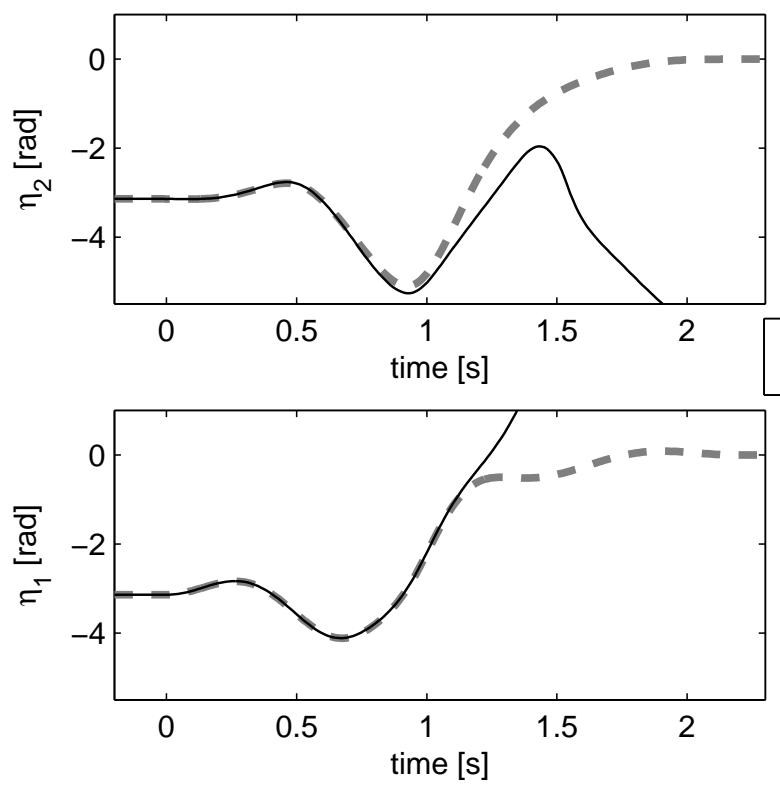

(b): adjusted parameters
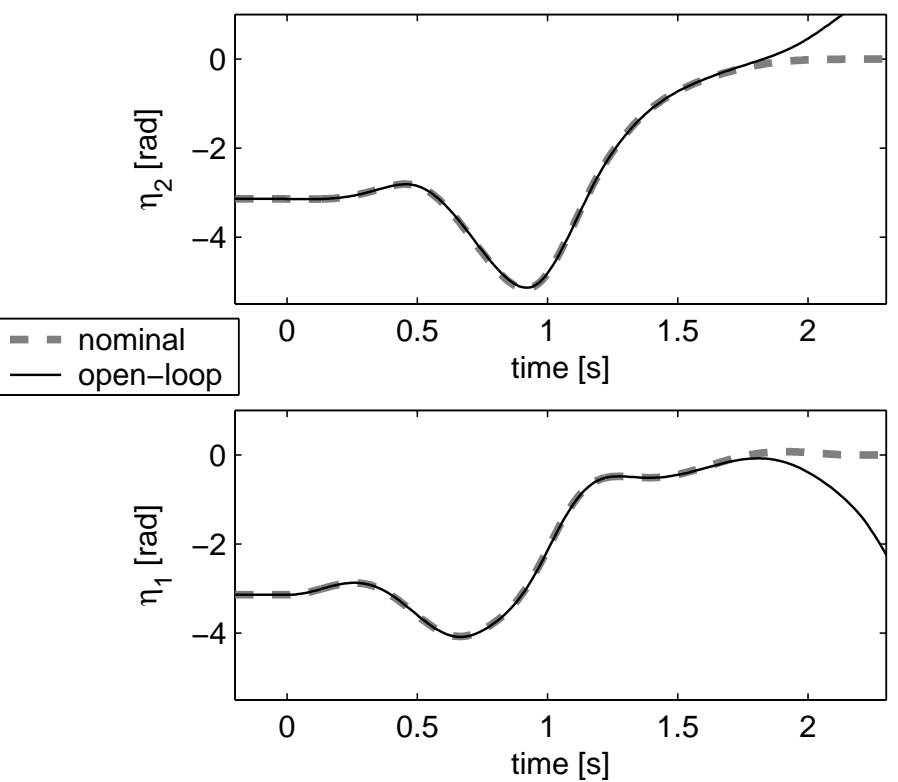

Figure 2.10: Nominal and experimental trajectories $\boldsymbol{\eta}^{*}(t)$ and $\boldsymbol{\eta}(t)$ for the swing-up of the double pendulum in open-loop mode with (a) default and (b) adjusted model parameters. 
the solution of the optimization problem (2.51)-(2.52) is not an identification of the mechanical parameters $\boldsymbol{\theta}$, but rather serves to fit the pendulum dynamics (2.52) to the measurement results of the open-loop swing-up maneuver.

The optimization problem is solved with the MATLAB function fmincon of the Optimization Toolbox. The adjusted parameters $\boldsymbol{\theta}$ are listed in Table 2.3, which are used together with the remaining default ones in Table 2.1 to recalculate the feedforward trajectories according to Section 2.4.2. Figure 2.10b shows the open-loop experimental results (-) for the swing-up with the feedforward trajectories $(--)$ based on the adjusted parameters. The accuracy of the second angle $\eta_{2}(t)$ is clearly improved and both angles follow the nominal trajectories $\eta_{1}^{*}(t)$, $\eta_{2}^{*}(t)$ close to the upward position. Although the difference between the nominal trajectories $\eta_{1}^{*}(t)$ and $\eta_{2}^{*}(t)$ in Figure 2.10a and 2.10b is almost negligible, the enhanced accuracy of the second angle $\eta_{2}(t)$ shows the high sensitivity of the swing-up problem with respect to the model parameters.

\begin{tabular}{lll}
\hline Pendulum link & $\begin{array}{l}\text { inner } \\
i=1\end{array}$ & $\begin{array}{l}\text { outer } \\
i=2\end{array}$ \\
\hline distance to center of gravity $a_{i}[\mathrm{~m}]$ & 0.186 & 0.195 \\
mass $m_{i}[\mathrm{~kg}]$ & 0.881 & 0.551 \\
moment of inertia $J_{i}\left[\mathrm{~N} \mathrm{~m} \mathrm{~s}^{2}\right]$ & 0.0141 & 0.0177 \\
friction constant $d_{i}[\mathrm{Nm} \mathrm{s}]$ & 0.0034 & 0.0016 \\
\hline
\end{tabular}

Table 2.3: Adjusted mechanical parameters for the swing-up of the double pendulum in Figure 2.10.

\subsubsection{Feedback control design}

The experimental realization of the swing-up maneuver requires a feedback control which stabilizes the double pendulum along the nominal trajectories. In order to compensate for a possible steady state error in the cart position $y$, the pendulum model (2.44)-(2.45) is dynamically extended by the disturbance model $\dot{\tilde{y}}=y$ with the new state $\tilde{y}$, which yields the overall state vector $\boldsymbol{x}=\left[y, \dot{y}, \boldsymbol{\eta}^{\top}, \dot{\boldsymbol{\eta}}^{\top}, \tilde{y}\right]^{\top} \in \mathbb{R}^{7}$. Hence, the system (2.44)-(2.45) together with the additional ODE $\dot{\tilde{y}}=y$ can be written in the form $\dot{\boldsymbol{x}}=\boldsymbol{f}(\boldsymbol{x}, u)$.

Due to the accuracy of the nonlinear feedforward control, the feedback part is designed with linear methods by linearizing the overall system $\dot{\boldsymbol{x}}=\boldsymbol{f}(\boldsymbol{x}, u)$ along the nominal trajectories $\boldsymbol{x}^{*}(t)=\left[y^{*}(t), \dot{y}^{*}(t), \boldsymbol{\eta}^{* \boldsymbol{\top}}(t), \dot{\boldsymbol{\eta}}^{* \boldsymbol{\top}}(t), \tilde{y}^{*}(t)\right]^{\boldsymbol{\top}}$ (with $\left.\tilde{y}^{*}(t)=\int_{0}^{t} y^{*}(\tau) \mathrm{d} \tau\right)$ and $u^{*}$. This leads to the linear time-varying system

$$
\Delta \dot{\boldsymbol{x}}=A(t) \Delta \boldsymbol{x}+\boldsymbol{b}(t) \Delta u
$$

with $A(t)=\left.\frac{\partial \boldsymbol{f}}{\partial \boldsymbol{x}}\right|_{\boldsymbol{x}^{*}(t), u^{*}(t)}$ and $\boldsymbol{b}(t)=\left.\frac{\partial \boldsymbol{f}}{\partial u}\right|_{\boldsymbol{x}^{*}(t), u^{*}(t)}$.

According to the two-degree-of-freedom control scheme in Figure 2.8, the closed-loop control

$$
u=u^{*}+\boldsymbol{k}^{\top}(t)\left(\boldsymbol{x}^{*}-\boldsymbol{x}\right)
$$


comprises the feedforward control $u^{*}(t)$ and the feedback part $\Delta u=\boldsymbol{k}^{\top}(t)\left(\boldsymbol{x}^{*}-\boldsymbol{x}\right)$. The calculation of the time-varying feedback gains $\boldsymbol{k}(t)$ is based on an optimal LQ (linear quadratic) feedback design which minimizes the objective functional

$$
I=\Delta \boldsymbol{x}^{\top}(T) M \Delta \boldsymbol{x}(T)+\int_{0}^{T}\left(\Delta \boldsymbol{x}^{\top} Q \Delta \boldsymbol{x}+\Delta u R \Delta u\right) \mathrm{d} t
$$

with the symmetric positive semidefinit matrices $M \in \mathbb{R}^{7 \times 7}, Q \in \mathbb{R}^{7 \times 7}$ and the positive scalar $R>0$. The solution $P(t), t \in[0, T]$ of the Riccati ODE, see e.g. (Kwakernaak and Sivan, 1972; Bertsekas, 2000),

$$
\dot{P}=-P A(t)-A^{\top}(t) P+P \boldsymbol{b}(t) R^{-1} \boldsymbol{b}^{\top}(t) P-Q, \quad P(T)=M
$$

determines the feedback gains

$$
\boldsymbol{k}^{\top}(t)=R^{-1} \boldsymbol{b}^{\top}(t) P(t) .
$$

The weighting matrices in $(2.55)$ are chosen to $Q=\operatorname{diag}(50,0,500,500,0,0,10)$ and $R=5$. The choice of the terminal condition $P(T)=M$ for the reverse-time integration of the Riccati equation (2.56) is a degree-of-freedom in the LQ-design. Thereby, the matrix $M \in \mathbb{R}^{7 \times 7}$ is determined by solving the algebraic Riccati equation following from $(2.56)$ with $\dot{P}(T)=0$. The terminal condition $M$ is determined using the MATLAB function lqr of the Control System Toolbox, whereas the reverse-time integration of (2.56) is performed with a standard ODE solver of MATLAB.

Figure 2.11a shows the time-varying feedback gains $k_{i}(t), i=1, \ldots, 7$ in the time interval $t \in[0, T]$ for the swing - up of the double pendulum with $T=2.2 \mathrm{~s}$. During the time interval $t \in$ $[0.5,1.2] \mathrm{s}$, the gains $k_{i}(t)$ change their signs several times and oscillate significantly. Although the LQ design provides optimal feedback gains $k_{i}(t)$ for minimizing the cost functional (2.55) over the time interval $t \in[0, T]$, the large gradients and magnitudes of the gains $k_{i}(t)$ for $t \in$ $[0.5,1.2]$ s pose significant demands on the closed-loop control of the double pendulum leading to large displacements of the cart position $y$. Moreover, the linear time-varying system (2.53)

(a) calculated

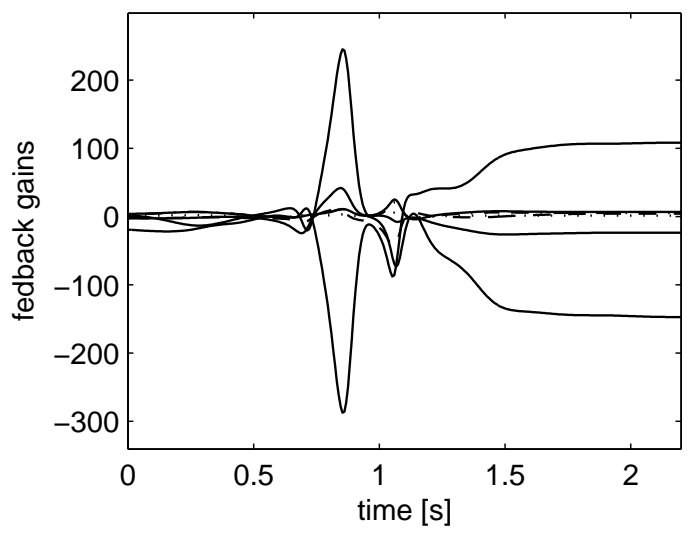

(b) implemented

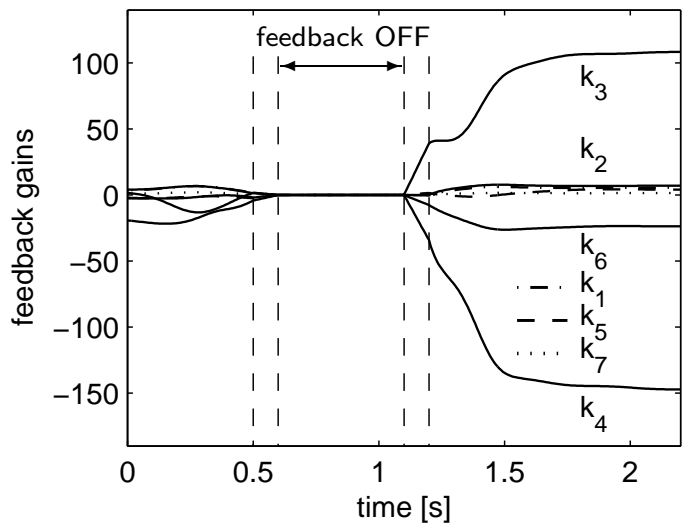

Figure 2.11: Time-varying LQ feedback gains $k_{i}(t), i=1, \ldots, 7$ for the swing-up maneuver of the double pendulum in the time $T=2.2 \mathrm{~s}$ : (a) calculated and (b) experimentally implemented values. 
looses its controllability several times in this time interval. ${ }^{10}$ Due to these reasons, the feedback control is turned off for $t \in[0.6,1.1] \mathrm{s}$ by setting the gain vector $\boldsymbol{k}(t)$ to zero. In the bordering intervals $t \in[0.5,0.6] \mathrm{s}$ and $t \in[1.1,1.2] \mathrm{s}$, the gains $k_{i}(t)$ are linearly interpolated between zero and the respective gains values at $t=0.5 \mathrm{~s}$ and $t=1.2 \mathrm{~s}$ in order to smoothly switch on/off the feedback control, see Figure 2.11b. Hence, during the time interval $t \in[0.6,1.1] \mathrm{s}$ the pendulum is steered along the nominal trajectories $\boldsymbol{x}^{*}(t)$ by the feedforward control without a stabilizing feedback control.

\subsubsection{Experimental results}

The implementation of the closed-loop control (2.54) requires full state information of the double pendulum. A Luenberger observer (O'Reilly, 1983) based on the nonlinear model (2.44)-(2.45) is used to estimate the state. The error dynamics of the observer is designed by eigenvalue assignment point-wise in time with the pendulum model linearized along the nominal trajectories, see (Treuer, 2005) for more details.

Figure 2.12 shows the experimental and nominal trajectories of the angles $\boldsymbol{\eta}(t)$, the cart $y(t)$, $\dot{y}(t)$, and the input $u(t)=\ddot{y}(t)$ for open-loop and closed-loop control of the swing-up maneuver. The open-loop trajectories reveal the good accuracy of the designed feedforward control $u^{*}(t)$ (also see Figure 2.10b), but the pendulum drifts away when it approaches the unstable upward position. In closed-loop mode, the feedback control is turned on at $t=1.1 \mathrm{~s}$ and stabilizes the pendulum along the nominal trajectories $\boldsymbol{x}^{*}(t)$. The feedback correction $\Delta u=\boldsymbol{k}^{T}(t)\left(\boldsymbol{x}^{*}-\boldsymbol{x}\right)$ in (2.54) is less than $3 \mathrm{~m} / \mathrm{s}^{2}$, which reveals the quality of the feedforward control $u^{*}(t)$ and the effectiveness of the parameter adjustment by solving the optimization problem $(2.51)-(2.52)$.

The experimental swing-up is easily repeatable without nameable performance loss after several successive swing-up and swing-down maneuvers (along the accordingly calculated swing-down trajectories). Although the double pendulum is a highly sensitive system, simulations and experiments have shown that the control scheme is robust enough to deal with minor deviations in the parameters and initial conditions.

\subsection{Conclusions}

In this chapter, the inversion-based feedforward control design has been introduced for the transition between stationary setpoints of nonlinear SISO systems. The offline design approach is based on the input-output representation of the system and treats the finite-time transition task as a two-point BVP throughout all design steps. The setup of the output trajectory contains a sufficient number of free parameters in order to solve the overdetermined BVP of the internal dynamics, e.g. with the standard MATLAB function bvp4c. The feedforward control

\footnotetext{
${ }^{10}$ See e.g. (Silverman and Meadows, 1967; Kailath, 1980) for the controllability of linear time-varying systems. The controllability of the linearized model (2.53) for the swing-up maneuver of the double pendulum is investigated in (Treuer, 2005).
} 

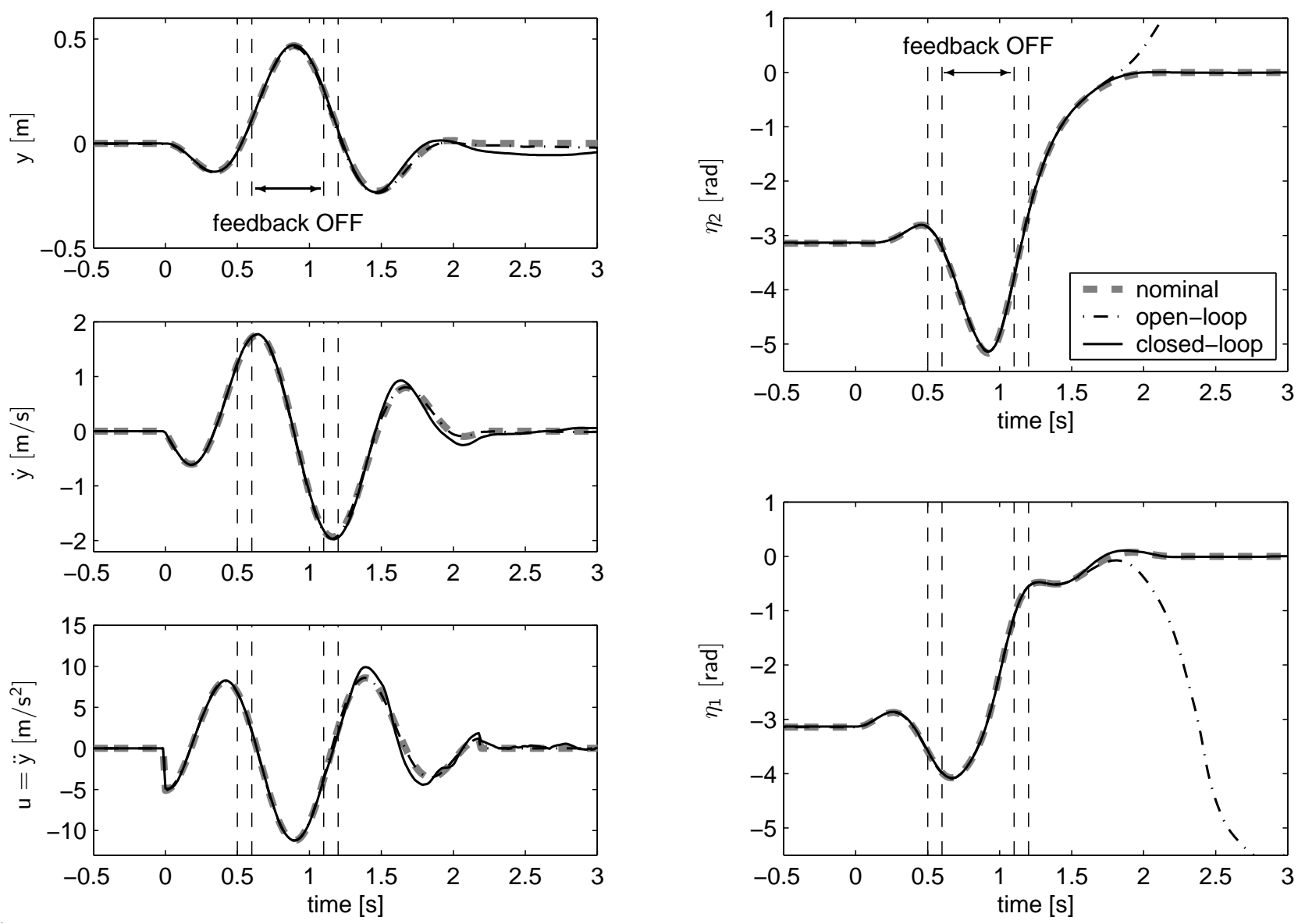

Figure 2.12: Experimental and nominal trajectories for the swing-up of the double pendulum in open/closed-loop with the swing-up time $T=2.2 \mathrm{~s}$.

design is applicable to both (non)linear and (non)minimum-phase systems, since no general restrictions are imposed on the considered system and algebraic BVP solution techniques like collocation (implemented in bvp4c) are not based on numerical integration and thus handle the BVP regardless of its stability. Moreover, the input and output trajectories $u^{*}(t)$ and $y^{*}(t)$ are constant outside the transition interval $t \in[0, T]$, since the stationary BCs ensure that the transition problem is performed in finite-time.

The application as well as the potential of the approach are illustrated for the swing-up problem of the double pendulum on a cart which combines both highly nonlinear dynamics and nonminimum-phase behavior. In (Treuer, 2005), the feedforward control design is also used to obtain nominal trajectories for the swing-up of the triple pendulum. However, the swing-up is only accomplished in simulation studies together with a state feedback controller with timevarying gains. The experimental validation of the triple pendulum swing-up is still an unsolved problem due to the high sensitivity and instability of the triple pendulum. 


\section{Chapter 3}

\section{Feedforward control design for nonlinear SISO systems with input constraints}

A common problem in many control applications is the presence of input constraints typically stemming from physical limitations, e.g. maximum available torque in mechatronic devices or limited cooling/heating power for chemical reactors. With respect to the two-degree-offreedom control scheme in Figure 1.1 (also see Figure 1.2), the SISO feedforward control $\Sigma_{F F}$ has to account for the constraints

$$
u^{*}(t) \in\left[u^{-}, u^{+}\right]
$$

Usually the limits $u^{-}$and $u^{+}$are chosen smaller than the physical constraints in order to leave sufficient control margin for the feedback control $\Sigma_{F B}$ in Figure 1.1. An evident assumption is that the input values $u_{0}^{*}$ and $u_{T}^{*}$ of the stationary setpoints (2.2) lie within the constraints, i.e. $u_{0, T}^{*} \in\left[u^{-}, u^{+}\right]$.

The inversion-based feedforward control design presented in the previous chapter only allows to consider the constraints (3.1) in a "manual" way, i.e. by checking the feedforward control (2.33), changing the transition time $T$ or using different types of setup functions (2.29), and resolving the BVP (2.32). In order to overcome this problem, this chapter considers the incorporation of input constraints within the formulation of the BVP by extending the BVP of the internal dynamics (2.27) by a further BVP for the output $y^{*}$. Moreover, the consideration of input constraints within the feedforward control design provides the opportunity to determine the transition time $T$ as part of the BVP solution in dependence of a desired aggressiveness of the feedforward control $u^{*}(t)$ with respect to the constraints (3.1). The feedforward control design under input constraints is illustrated for a setpoint change scenario of a continuous stirred tank reactor (CSTR). 


\subsection{Boundary value problems for constrained input}

In order to directly account for the input constraints (3.1), the feedforward control (2.26) reveals that $u^{*}$ can only be directly affected by the highest time derivative $y^{*(r)}$ of the output, since the remaining derivatives $y^{*(i)}, i=1, \ldots, r-1$ and $y^{*}(t)$ can be solved by integration and the state $\boldsymbol{\eta}^{*}$ is determined by the BVP (2.27)-(2.28) of the internal dynamics. Hence, a new setup function $\hat{\alpha}=y^{*(r)}$ is introduced to parameterize the highest time derivative of the output (Graichen and Zeitz, 2005b, 2006c). This extends the previous BVP (2.27)-(2.28) of the internal dynamics by a further BVP for the output $y^{*}$ :

$$
\begin{aligned}
& y^{*(r)}=\hat{\alpha}, \\
& y^{*}(0)=y_{0}^{*}, \quad y^{*}(T)=y_{T}^{*},\left.\quad y^{*(i)}\right|_{t=0, T}=0, \quad i=1, \ldots, r-1 \\
& \dot{\boldsymbol{\eta}}^{*}=\overline{\boldsymbol{\beta}}\left(\boldsymbol{\eta}^{*}, y^{*}, \dot{y}^{*}, \ldots, y^{*(r-1)}, \hat{\alpha}\right), \quad \boldsymbol{\eta}^{*}(0)=\boldsymbol{\eta}_{0}^{*}, \quad \boldsymbol{\eta}^{*}(T)=\boldsymbol{\eta}_{T}^{*} .
\end{aligned}
$$

The solutions $y^{*}(t)$ and $\boldsymbol{\eta}^{*}(t)$ of the two BVPs (3.2)-(3.3) and the feedforward control $u^{*}(t)$ in (2.26) depend on the setup of the function $\hat{\alpha}=y^{*(r)}$ as described in the following points:

(i) If it is desired that the feedforward trajectory $u^{*}(t)$ in $(2.26)$ is continuous at the bounds $t=0$ and $t=T$, the output trajectory $y^{*}(t)$ must meet the additional BCs in (2.20) for $\rho=r$, which are repeated here for the sake of completeness:

$$
y^{*(r)}(0)=0, \quad y^{*(r)}(T)=0 .
$$

These BCs have to be satisfied by the function $\hat{\alpha}=y^{*(r)}$ if continuity of the feedforward control $u^{*}(t)$ is desired.

(ii) The solvability of the BVPs (3.2)-(3.3) defined by $n$ first-order ODEs and $2 n$ BCs requires $n$ free parameters. Similar to the procedure in the last chapter, the parameters $\boldsymbol{p}=$ $\left(p_{1}, \ldots, p_{n}\right)$ are provided in a setup function $\Phi(t, \boldsymbol{p})$, which is used to parameterize $\hat{\alpha}=$ $\Phi(t, \boldsymbol{p})$ if the corresponding feedforward control $(2.26)$

$$
u_{\Phi}^{*}=\alpha^{-1}\left(y^{*}, \ldots, y^{*(r-1)}, \Phi(t, \boldsymbol{p}), \boldsymbol{\eta}^{*}\right)
$$

stays within the constraints $(3.1)$, i.e. $u_{\Phi}^{*} \in\left[u^{-}, u^{+}\right]$.

\begin{tabular}{|c|c|c|c|c|}
\hline & polynomial & & trigonometric series & \\
\hline $\begin{array}{l}\Phi(t, \boldsymbol{p}): \\
\text { no BCs }\end{array}$ & $\Phi(t, \boldsymbol{p})=\sum_{i=1}^{n} p_{i}\left(\frac{t}{T}\right)^{i-1}$ & $(3.6 \mathrm{a})$ & $\Phi(t, \boldsymbol{p})=\sum_{i=1}^{n} p_{i} \cos \frac{(i-1) \pi t}{T}$ & $(3.6 \mathrm{~b})$ \\
\hline $\begin{array}{l}\Phi(t, \boldsymbol{p}): \\
\left.\Phi\right|_{0, T}=0\end{array}$ & $\Phi(t, \boldsymbol{p})=\sum_{i=1}^{n} p_{i}\left[\left(\frac{t}{T}\right)^{i+1}-\frac{t}{T}\right.$ & $(3.6 \mathrm{c})$ & $\Phi(t, \boldsymbol{p})=\sum_{i=1}^{n} p_{i} \sin \frac{i \pi t}{T}$ & $(3.6 \mathrm{~d})$ \\
\hline
\end{tabular}

(iii) The function $\Phi(t, \boldsymbol{p})$ has to satisfy the BCs $(3.4)$, i.e. $\Phi(0, \boldsymbol{p})=\Phi(T, \boldsymbol{p})=0$ to achieve continuity of the feedforward control $u^{*}(t)$. The equation array (3.6) lists convenient choices for $\Phi(t, \boldsymbol{p})$ with polynomial or trigonometric basis functions and with/without consideration of the homogeneous BCs (3.4) for $\Phi(t, \boldsymbol{p})$. 
The equations (3.6a) and (3.6b) do not account for the BCs (3.4) in contrast to the setups (3.6c) and (3.6d), which directly satisfy the homogeneous BCs $\Phi(0, \boldsymbol{p})=\Phi(T, \boldsymbol{p})=0$ thus resulting in a continuous feedforward trajectory $u^{*}(t)$ at the bounds $t=0$ and $t=T$. For many higher order systems, e.g. $n>6$, the trigonometric functions (3.6b) and (3.6d) have proven to be numerically more robust than the polynomial setup. This is mainly due to the fact that the polynomial coefficients in (3.6a) and (3.6c) increase significantly in magnitude for higher order terms.

(iv) If the feedforward control $u_{\Phi}^{*}$ in (3.5) for $\hat{\alpha}=\Phi(t, \boldsymbol{p})$ is outside the bounds $u^{-}$and $u^{+}$, the input-output dynamics (2.18) is used to replan the right-hand side $\hat{\alpha}$ of ODE (3.2) such that the constraints (3.1) are met. This is accomplished by the case-dependent definition ${ }^{1}$

$$
\hat{\alpha}= \begin{cases}\Phi(t, \boldsymbol{p}) & \text { if } u_{\Phi}^{*} \in\left[u^{-}, u^{+}\right] \\ \alpha\left(y^{*}, \ldots, y^{*(r-1)}, \boldsymbol{\eta}^{*}, u^{-}\right) & \text {if } u_{\Phi}^{*}<u^{-} \\ \alpha\left(y^{*}, \ldots, y^{*(r-1)}, \boldsymbol{\eta}^{*}, u^{+}\right) & \text {if } u_{\Phi}^{*}>u^{+} .\end{cases}
$$

Due to the case-dependent choice of $\hat{\alpha}$ in (3.7), the input constraints are directly incorporated in the formulation of the BVPs. Thereby, the "inversion-based" design of the feedforward control is preserved by planning the highest output derivative $y^{*(r)}=\hat{\alpha}$ such that input constraints (3.1) are satisfied. The solution of the two BVPs (3.2)-(3.3) with (3.5)-(3.7) comprises the trajectories $y^{*(i)}(t), i=0,1, \ldots, r-1$ and $\boldsymbol{\eta}^{*}(t), t \in[0, T]$ as well as the $n$ free parameters in the set $\boldsymbol{p}$. The feedforward control $u^{*}(t)$ finally follows from (2.26), whereby the highest output derivative $y^{*(r)}=\hat{\alpha}$ as defined by (3.7) can be calculated with the trajectories $y^{*(i)}(t), i=$ $0,1, \ldots, r-1$ and $\boldsymbol{\eta}^{*}(t)$.

Remark 3.1. By means of the function $\hat{\alpha}$ in (3.7), the input constraints (3.1) are mapped to the respective limits

$$
\begin{aligned}
& \alpha^{-}=\min \left\{\quad \alpha\left(y^{*}, \ldots, y^{*(r-1)}, \boldsymbol{\eta}^{*}, u^{-}\right), \alpha\left(y^{*}, \ldots, y^{*(r-1)}, \boldsymbol{\eta}^{*}, u^{+}\right)\right\}, \\
& \alpha^{+}=\max \left\{\alpha\left(y^{*}, \ldots, y^{*(r-1)}, \boldsymbol{\eta}^{*}, u^{-}\right), \quad \alpha\left(y^{*}, \ldots, y^{*(r-1)}, \boldsymbol{\eta}^{*}, u^{+}\right)\right\}
\end{aligned}
$$

for the highest output derivative $y^{*(r)}$ depending on the current states $y^{*(i)}, i=0,1, \ldots, r-1$ and $\boldsymbol{\eta}^{*}$, i.e. $\alpha^{ \pm}=\alpha^{ \pm}\left(y^{*}, \ldots, y^{*(r-1)}, \boldsymbol{\eta}^{*}\right)$. Thereby, the projection of the input constraints $u^{*} \in$ $\left[u^{-}, u^{+}\right]$to the highest output derivative $y^{*(r)}=\hat{\alpha} \in\left[\alpha^{-}, \alpha^{+}\right]$is unique due to the assumption that the input-output dynamics (2.18) is invertible with respect to $u$, i.e. $\partial \alpha / \partial u \neq 0$ holds for $u \in\left[u^{-}, u^{+}\right]$. Hence, the definition (3.7) is equivalent to

$$
\hat{\alpha}= \begin{cases}\Phi(t, \boldsymbol{p}) & \text { if } \Phi \in\left[\alpha^{-}, \alpha^{+}\right] \\ \alpha^{-}\left(y^{*}, \ldots, y^{*(r-1)}, \boldsymbol{\eta}^{*}\right) & \text { if } \Phi<\alpha^{-} \\ \alpha^{+}\left(y^{*}, \ldots, y^{*(r-1)}, \boldsymbol{\eta}^{*}\right) & \text { if } \Phi>\alpha^{+} .\end{cases}
$$

\footnotetext{
${ }^{1}$ The setup $\Phi(t, \boldsymbol{p})$ always determines the function $\hat{\alpha}=\Phi(t, \boldsymbol{p})$ at the interval bounds $t=0, T$, since $u_{\Phi}^{*}(0, \boldsymbol{p})=u_{0}^{*}$ and $u_{\Phi}^{*}(T, \boldsymbol{p})=u_{T}^{*}$ (see $\left.(2.2)\right)$ must lie within the interval $\left[u^{-}, u^{+}\right]$. Hence, the two additional BCs in (3.4) are always satisfied by $\Phi(t, \boldsymbol{p})$.
} 
However, (3.7) requires only one evaluation of (3.5) if $u_{\Phi}^{*} \in\left[u^{-}, u^{+}\right]$holds, whereas (3.9) always requires two function evaluations to determine the limits (3.8). Moreover, the definition of $\hat{\alpha}$ according to (3.7) can be extended to the MIMO case in a straightforward manner, see Chapter 5.

Example 3.1 (Examples 2.3-2.4 continued). In order to incorporate the input constraints (3.1) for the linear SISO system in Example 2.3, the internal dynamics BVP (2.35) is augmented by a further $B V P$ for the output $y^{*}$ with relative degree $r=1$ :

$$
\begin{aligned}
& \dot{y}^{*}=\hat{\alpha}, \quad y^{*}(0)=y_{0}^{*}, \quad y^{*}(T)=y_{T}^{*} \\
& \dot{\eta}^{*}=-a_{0} y^{*}-\frac{b_{0}}{b_{1}}\left(a_{1} y^{*}+\hat{\alpha}-\eta^{*}\right)=\bar{\beta}\left(\eta^{*}, y^{*}, \hat{\alpha}\right), \quad \eta^{*}(0)=\eta_{0}^{*}, \quad \eta^{*}(T)=\eta_{T}^{*} .
\end{aligned}
$$

The BVPs are overdetermined by $2 n=4 B C$ s for $n=2$ ODEs. Hence, two free parameters $\boldsymbol{p}=\left(p_{1}, p_{2}\right)$ are provided in the setup function $\Phi(t, \boldsymbol{p})$ by using the polynomial (3.6c) to satisfy the additional BCs $\dot{y}^{*}(0)=\dot{y}^{*}(T)=0$. The setup function $\hat{\alpha}$ follows from (3.7) with the input-output dynamics (2.22) and the feedforward control (2.34).

The BVPs (3.10) are solved with bvp4c. The initial guess for the trajectories $y^{*}\left(t_{k}\right)$ and $\eta^{*}\left(t_{k}\right)$ is a linear interpolation between the corresponding BCs on a uniform time mesh $t_{k} \in[0, T], k=$ $1, \ldots, 30$. The initial guess of the free parameter is $\boldsymbol{p}=\mathbf{0}$. Finally, the feedforward control $u^{*}(t) \in\left[u^{-}, u^{+}\right]$follows from (2.34) with the solutions $y^{*}(t)$ and $\eta^{*}(t), t \in[0, T]$ returned by bvp4c.
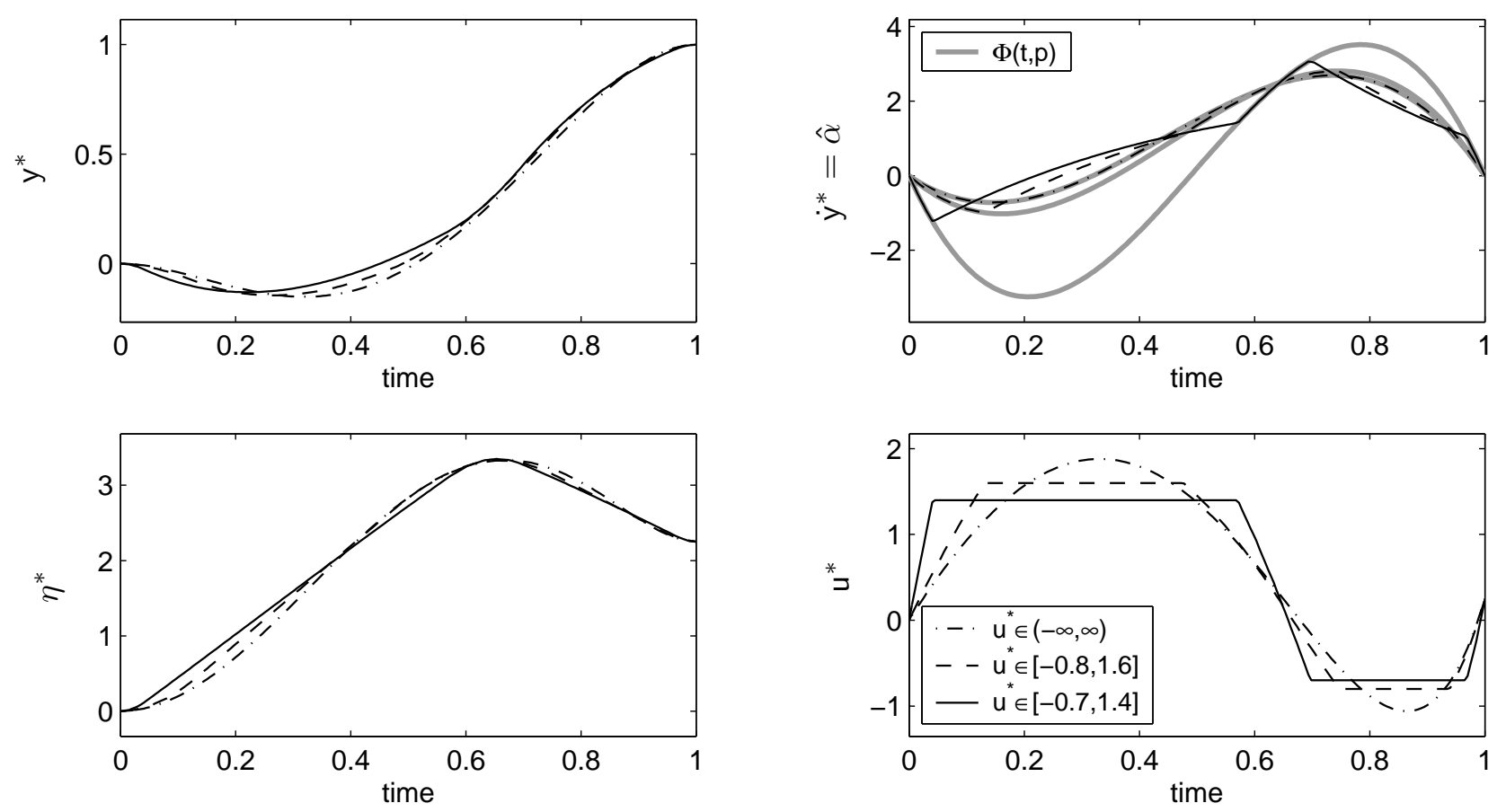

Figure 3.1: Setpoint transition $y_{0}^{*}=0 \rightarrow y_{T}^{*}=1$ with $T=1$ of the linear SISO system (1.1) based on the solution of the BVPs (3.10) with the parameters (2.15) and various input constraints $u^{*}(t) \in\left[u^{-}, u^{+}\right]$. 
Figure 3.1 shows the nominal trajectories $\eta^{*}(t), y^{*}(t)$, and $u^{*}(t)$ for the setpoint transition $y_{0}^{*}=0 \rightarrow y_{T}^{*}=1$ with $T=1$ and three different input constraints. The boundary values (2.25) for $\eta^{*}(t)$ are $\eta_{0}^{*}=0$ and $\eta_{T}^{*}=2.25$. For the strictest constraints $u^{*}(t) \in[-0.7,1.4]$, the feedforward control $u^{*}(t)$ approaches a bang-bang behavior by hitting both constraints $u^{-}=-0.7$ and $u^{+}=1.4$. In these time intervals, $\dot{y}^{*}=\hat{\alpha}$ is replanned according to (3.7) by means of the input-output dynamics (2.22), see Figure 3.1. In the unconstrained case $\left|u^{*}(t)\right|<\infty$, the trajectories $y^{*}(t), \eta^{*}(t)$, and $u^{*}(t)$ are the same as in Figure 2.2 for $T=1$. Since the righthand side of the ODE in (3.10a) is given by $\hat{\alpha}=\Phi(t, \boldsymbol{p})$ for the whole time interval $t \in[0, T]$, its integration yields a polynomial output trajectory $y^{*}(t)$ corresponding to the setup $y^{*}(t)=\Upsilon(t, p)$ in (2.36).

\subsection{Calculation of transition time}

The transition time $T$ is of particular importance for the feedforward control design in the presence of input constraints. The feedforward control $u^{*}(t)$ will approach a bang-bang behavior if $T$ is reduced to a minimal realizable value $T_{\min }$ that individually depends on the system dynamics (2.1), the setpoints (2.2), and the input constraints (3.1). However, no solution of the BVPs (3.2)-(3.7) exists if $T$ is chosen smaller than the respective minimum $T_{\min }$. Hence, the transition time $T$ has to be determined appropriately for the considered transition problem (possibly in an iterative manner) and with respect to the desired aggressiveness of the transition.

To simplify this problem, this section describes an approach to determine the transition time $T$ as part of the BVP solution. Thereby, a normalized design parameter $\delta \in(0,1)$ serves as aggressiveness measure for the feedforward control $u^{*}(t)$ in order to rate the desired exploitation of the input constraints (3.1) (Graichen and Zeitz, 2005b). A necessary condition to apply this ratio is the existence of a quasi-stationary connection between the two stationary setpoints (2.2), i.e. a connected set of equilibria between $\boldsymbol{x}_{0}^{*}$ and $\boldsymbol{x}_{T}^{*}$. By choosing $\delta \rightarrow 0$, the system shall approach the quasi-stationary connection, which corresponds to a large transition time $T{ }^{2}$ For $\delta \rightarrow 1$, the feedforward control $u^{*}(t)$ shall exploit the input constraints (3.1) in a bang-bang like behavior leading to a nearly time-optimal transition.

\subsubsection{Aggressiveness measure for the feedforward control}

A convenient way to rate the aggressiveness of the feedforward control $u^{*}(t)$ is by means of the highest output derivative $y^{*(r)}=\hat{\alpha}$ in (3.2). The advantage of rating the aggressiveness of the setup function $\hat{\alpha}$ instead of $u^{*}(t)$ is that for large transition times $T$ the output trajectory $y^{*}(t)$ will approach a quasi-stationary connection between the setpoints $y_{0}^{*}$ and $y_{T}^{*}$, whereby the time derivatives $y^{*(i)}(t) \rightarrow 0, i=1, \ldots, r$ will tend to zero.

\footnotetext{
${ }^{2}$ For instance, the double pendulum on cart in Section 2.4 possesses no quasi-stationary connection between the downward and upward equilibria. Hence, the pendulum swing-up cannot be performed arbitrarily slowly, also see (Graichen and Zeitz, 2005a).
} 

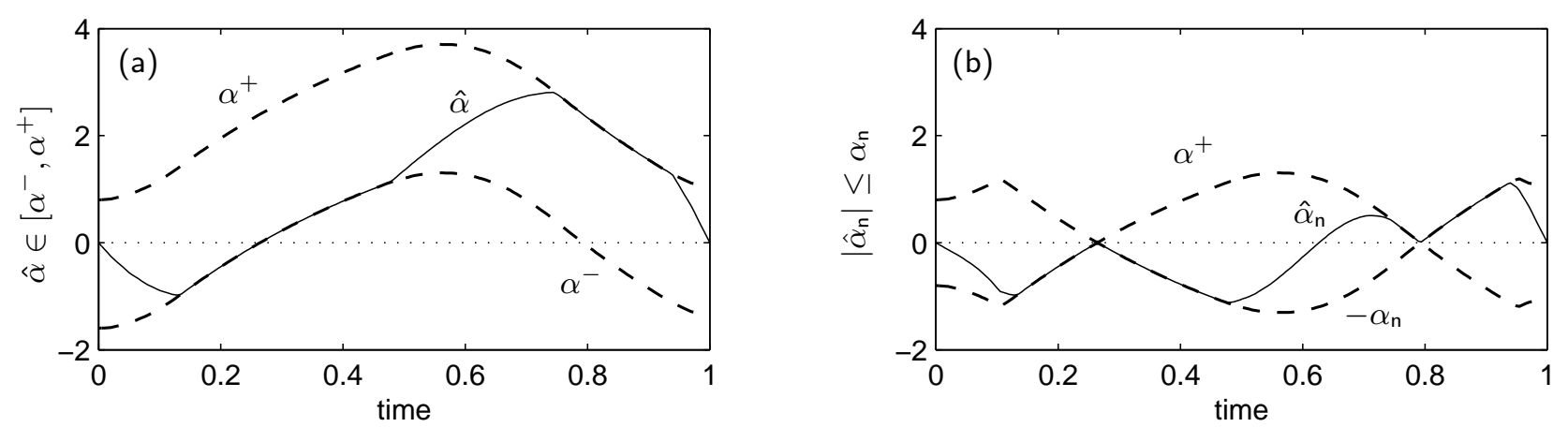

Figure 3.2: Trajectories $y^{*(r)}=\hat{\alpha} \in\left[\alpha^{-}, \alpha^{+}\right]$in (a) and corresponding normalized ones $\left|\hat{\alpha}_{\mathrm{n}}\right| \leq \alpha_{\mathrm{n}}$ in (b) for the setpoint transition in Example 3.1 with input constraints $u^{*} \in[-0.8,1.6]$.

The case-dependent definition (3.7) projects the input constraints $u^{*} \in\left[u^{-}, u^{+}\right]$to the constraints (3.8) for the setup function $y^{*(r)}=\hat{\alpha} \in\left[\alpha^{-}, \alpha^{+}\right]$with respect to the current states $y^{*(i)}, i=0,1, \ldots, r-1$ and $\boldsymbol{\eta}^{*}$. Figure 3.2 a shows the trajectory of $y^{*(r)}=\hat{\alpha}$ for the setpoint transition in Figure 3.1 (Example 3.1) with $T=1$ and the input constraints $u^{*} \in[-.8,1.6]$ as well as the corresponding limits $\alpha^{-}, \alpha^{+}, t \in[0, T]$ obtained from (3.8) with the nominal trajectories $y^{*}(t)$ and $\eta^{*}(t)$.

The aggressiveness of the feedforward control $u^{*}(t)$ can be rated by comparing the area between the curve $\hat{\alpha}$ and the time axis with the maximum available area under $\alpha^{-}$or $\alpha^{+}$(depending on the sign of $\hat{\alpha}$ ). Thereby, the time axis, i.e. $\hat{\alpha}=0$, is used as reference since it represents the quasi-stationary connection with $y^{*(r)}=\hat{\alpha} \rightarrow 0$ for a sufficiently large transition time $T$. If the feedforward control $u^{*}(t)$ hits the constraints $u^{-}$or $u^{+}$, the highest time derivative $y^{*(r)}=\hat{\alpha}$ also touches the respective limit $\alpha^{-}$or $\alpha^{+}$, respectively, see Figure 3.2a. During these time intervals, the areas between the time axis and the curve $\hat{\alpha}$ as well as between the time axis and the respective limit $\alpha^{-}$or $\alpha^{+}$are the same. The time axis $\hat{\alpha}=0$ can always be used as reference to evaluate the ares under the curves $\hat{\alpha}$ and the limits $\alpha^{-}, \alpha^{+}$for a sufficiently large transition time $T$. In this case, the output derivatives $y^{*(i)} \rightarrow 0, i=1, \ldots, r$ tend to zero and it is ensured that the limits $\alpha^{-}<0<\alpha^{+}$enclose the time axis.

However, a fast transition with a correspondingly small transition time $T$ may lead to the problem that the limits $\alpha^{-}, \alpha^{+}$cross the time axis such that $0 \notin\left[\alpha^{-}, \alpha^{+}\right]$holds for a certain time interval (see Figure 3.2a). In this case, the time axis cannot be used as reference to evaluate the areas under the curve $\hat{\alpha}$ and the respective limit $\alpha^{-}$or $\alpha^{+}$. Therefore, the function $\hat{\alpha} \in\left[\alpha^{-}, \alpha^{+}\right]$has to be transformed appropriately. In a first step, the limits $\alpha^{-}$and $\alpha^{+}$are symmetrically mapped to the normalized values $\pm \alpha_{\mathrm{n}}$ with

$$
\alpha_{\mathrm{n}}=\min \left\{\left|\alpha^{-}\right|,\left|\alpha^{+}\right|\right\}
$$

This ensures that the time axis lies within the normalized limits $-\alpha_{\mathrm{n}} \leq 0 \leq \alpha_{\mathrm{n}}$, see Figure 3.2b. Moreover, the function $\hat{\alpha} \in\left[\alpha^{-}, \alpha^{+}\right]$has to be mapped to $\hat{\alpha}_{n}$ with $\left|\hat{\alpha}_{n}\right| \leq \alpha_{n}$. If the time axis 

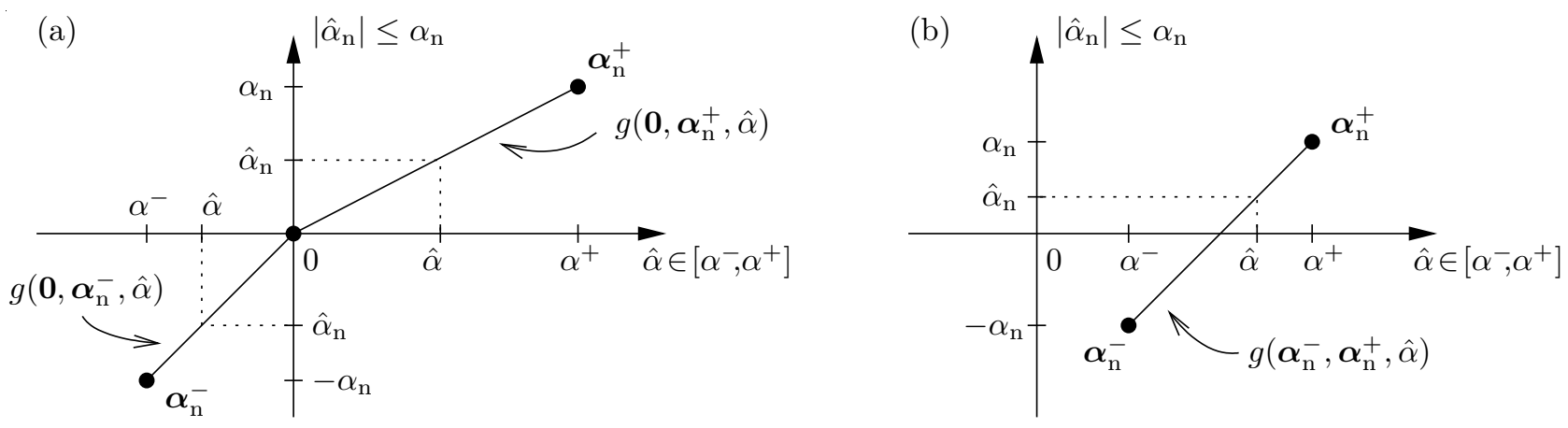

Figure 3.3: Normalized value $\hat{\alpha}_{\mathrm{n}} \in\left[\alpha_{\mathrm{n}}^{-}, \alpha_{\mathrm{n}}^{+}\right]$of the function $y^{*(r)}=\hat{\alpha} \in\left[\alpha^{-}, \alpha^{+}\right]$calculated with the linear interpolation $g(\cdot, \cdot, \hat{\alpha})$ for the cases a) $\alpha^{-} \leq 0 \leq \alpha^{+}$in $(3.14 \mathrm{a})-(3.14 \mathrm{~b})$ and b) $0 \notin\left[\alpha^{-}, \alpha^{+}\right]$in $(3.14 \mathrm{c})$.

lies within the interval $\alpha^{-} \leq 0 \leq \alpha^{+}$, the normalized value $\hat{\alpha}_{\mathrm{n}}$ is determined via a linear interpolation function $\hat{\alpha}_{\mathrm{n}}=g\left(\mathbf{0}, \boldsymbol{\alpha}_{\mathrm{n}}^{ \pm}, \hat{\alpha}\right)$ between the origin $\mathbf{0}=(0,0)$ and one of the points

$$
\boldsymbol{\alpha}_{\mathrm{n}}^{-}=\left(\alpha^{-},-\alpha_{\mathrm{n}}\right), \quad \boldsymbol{\alpha}_{\mathrm{n}}^{+}=\left(\alpha^{+}, \alpha_{\mathrm{n}}\right)
$$

depending on the sign of $\hat{\alpha}$, see Figure $3.3 \mathrm{a} .{ }^{3}$ If the time axis is outside the interval $\left[\alpha^{-}, \alpha^{+}\right]$, i.e. $\alpha^{-}>0$ or $\alpha^{+}<0$, the normalized value $\hat{\alpha}_{\mathrm{n}}$ is determined by the linear interpolation $\hat{\alpha}_{\mathrm{n}}=g\left(\boldsymbol{\alpha}_{\mathrm{n}}^{-}, \boldsymbol{\alpha}_{\mathrm{n}}^{+}, \hat{\alpha}\right)$ between both points (3.12), see Figure 3.3b. These three alternatives yield the case-dependent definition

$$
\hat{\alpha}_{\mathrm{n}}= \begin{cases}g\left(\mathbf{0}, \boldsymbol{\alpha}_{\mathrm{n}}^{-}, \hat{\alpha}\right) & \text { if } 0 \in\left[\alpha^{-}, \alpha^{+}\right] \text {and } \hat{\alpha} \leq 0 \\ g\left(\mathbf{0}, \boldsymbol{\alpha}_{\mathrm{n}}^{+}, \hat{\alpha}\right) & \text { if } 0 \in\left[\alpha^{-}, \alpha^{+}\right] \text {and } \hat{\alpha}>0 \\ g\left(\boldsymbol{\alpha}_{\mathrm{n}}^{-}, \boldsymbol{\alpha}_{\mathrm{n}}^{+}, \hat{\alpha}\right) & \text { if } 0 \notin\left[\alpha^{-}, \alpha^{+}\right]\end{cases}
$$

The cases (3.14a) and (3.14b) ensure that the time axis $\hat{\alpha}=0$ stays at $\hat{\alpha}_{\mathrm{n}}=0$ if $\alpha^{-} \leq 0 \leq \alpha^{+}$ holds. This is of importance to ensure that if $y^{*(r)}=\hat{\alpha} \rightarrow 0$ holds for a large transition time $T$, the normalized value $\hat{\alpha}_{\mathrm{n}}$ also approaches $\hat{\alpha}_{\mathrm{n}} \rightarrow 0$. Figure $3.2 \mathrm{~b}$ shows the normalized profiles $\hat{\alpha}_{\mathrm{n}}$ and $\pm \alpha_{\mathrm{n}}$ corresponding to Figure 3.2a.

\subsubsection{Boundary value problem for feedforward aggressiveness}

Based on the definition (3.14), the areas under the curves $\left|\hat{\alpha}_{n}\right|$ and $\alpha_{n}$ can be determined by integrating the two ODEs

$$
\dot{\omega}_{1}=\left|\hat{\alpha}_{\mathrm{n}}\right|, \quad \dot{\omega}_{2}=\alpha_{\mathrm{n}}, \quad \omega_{1}(0)=\omega_{2}(0)=0,
$$

\footnotetext{
${ }^{3}$ For a linear interpolation between two given points $\boldsymbol{Q}=\left(q_{1}, q_{2}\right)$ and $\boldsymbol{R}=\left(r_{1}, r_{2}\right)$, the ordinate coordinate of a point $\boldsymbol{S}=\left(s_{1}, s_{2}\right)$ is determined according to

$$
s_{2}=g\left(\boldsymbol{Q}, \boldsymbol{R}, s_{1}\right)=\frac{q_{2} r_{1}-q_{1} r_{2}}{r_{1}-q_{1}}+\frac{r_{2}-q_{2}}{r_{1}-q_{1}} s_{1} .
$$


which extend the previous BVPs (3.2)-(3.3). Thereby, $\omega_{1}(T)$ and $\omega_{2}(T)$ denote the areas under the respective curves. Since $\left|\hat{\alpha}_{n}\right| \leq \alpha_{\mathrm{n}}$ is ensured, the ratio $\omega_{1}(T) / \omega_{2}(T)$ between the two areas lies within the interval $(0,1)$. This ratio can be set to a desired value by introducing the design parameter $\delta \in(0,1)$ and demanding the terminal $\mathrm{BC}$

$$
\omega_{1}(T)-\delta \omega_{2}(T)=0
$$

to be satisfied. For $\delta \rightarrow 1$, both curves $\left|\hat{\alpha}_{n}\right|$ and $\alpha_{n}$ approach the same area values $\omega_{1}(T)$ and $\omega_{2}(T)$, which signifies an aggressive transition leading to a bang-bang behavior of the feedforward control $u^{*}(t)$ in a nearly time-optimal transition time $T$. On the other hand, $\delta \rightarrow 0$ means that the surface $\omega_{1}(T)$ under the curve $\left|\hat{\alpha}_{n}\right|$ tends to zero compared to the maximum available area under $\left|\alpha_{\mathrm{n}}\right|$, i.e. $y^{*(r)}=\hat{\alpha} \rightarrow 0$ for the whole time interval $t \in[0, T]$. The feedforward control $u^{*}(t)$ and the trajectories $y^{*}(t), \boldsymbol{\eta}^{*}(t)$ thus approach a quasi-stationary connection with a corresponding large transition time $T$.

The discussion shows that the design parameter $\delta \in(0,1)$ can be used to influence the transition time $T$ by a desired aggressiveness of the feedforward control $u^{*}(t)$. Since the previous BVPs (3.2)-(3.3) are extended by the two ODEs for $\omega_{1}(t), \omega_{2}(t)$ and three BCs in (3.15) and (3.16), another free parameter is required besides $\boldsymbol{p}=\left(p_{1}, \ldots, p_{n}\right)$. The transition time $T$ can be incorporated as free parameter by using the time transformation

$$
t=\varepsilon \tau, \quad T=\varepsilon, \quad \frac{\mathrm{d}^{i}}{\mathrm{~d} t^{i}}=\frac{1}{\varepsilon^{i}} \frac{\mathrm{d}^{i}}{\mathrm{~d} \tau^{i}}, \quad i \geq 1
$$

with the normalized time coordinate $\tau \in[0,1]$ and the scaling factor $\varepsilon$. Applying this time transformation to the BVPs (3.2)-(3.3) and (3.15)-(3.16) results in a fixed time interval $\tau \in$ $[0,1]$ with the scaling factor $\varepsilon=T$ as the new parameter, which can be influenced by the aggressiveness measure $\delta \in(0,1)$.

Remark 3.2. The ODEs (3.15) rate the areas under the curves $\left|\hat{\alpha}_{\mathrm{n}}\right|$ and $\alpha_{\mathrm{n}}$. Alternatively, the $O D E s$

$$
\dot{\omega}_{1}=\hat{\alpha}_{\mathrm{n}}^{2}, \quad \dot{\omega}_{2}=\alpha_{\mathrm{n}}^{2}, \quad \omega_{1}(0)=\omega_{2}(0)=0
$$

can be used, where $\omega_{1}(T) / \omega_{2}(T)$ weights the volume of both curves $\hat{\alpha}_{\mathrm{n}}(t)$ and $\alpha_{\mathrm{n}}(t)$ rotating around the time axis. If analytical Jacobians are provided for the numerical solution of the BVP (see Section 2.3.2), the ODEs (3.18) have the advantage that the respective Jacobians are continuous. In the case of (3.15), the partial derivatives of $\left|\hat{\alpha}_{n}\right|$ with respect to the states $y^{*(i)}, i=0,1, \ldots, r$ and $\boldsymbol{\eta}^{*}$ are discontinuous at $\hat{\alpha}_{\mathrm{n}}=0$.

Example 3.2 (Example 3.1 continued). The BVPs (3.10) of the linear SISO system together with the case-dependent definition (3.7), the input-output dynamics (2.22), and the feedforward control (2.34) are extended by the ODEs (3.15) or respectively (3.18), in order to rate the aggressiveness of the feedforward control $u^{*}(t) \in\left[u^{-}, u^{+}\right]$by the ratio $\omega_{1}(T) / \omega_{2}(T) \stackrel{!}{=} \delta$. Thereby, the time transformation (3.17) is used to treat the transition time $\varepsilon=T$ as the third free parameters besides $\boldsymbol{p}=\left(p_{1}, p_{2}\right)$ in the setup function $\Phi(t, \boldsymbol{p})$. 
The BVPs (3.10) together with the ODEs (3.15) (or (3.18), respectively) and the terminal BCs (3.16) are solved with bvp4c. As mentioned in Example 3.1, the initial guess for the parameters is $\boldsymbol{p}=\mathbf{0}$ and a linear interpolation between the respective BCs is used for the trajectories $y^{*}\left(t_{k}\right)$ and $\eta^{*}\left(t_{k}\right)$. The initial guess for the additional states is $\omega_{1}\left(t_{k}\right)=\omega_{2}\left(t_{k}\right)=0$. A reasonable value for the initial guess of the free transition time is $\varepsilon=3$. Figure 3.4 shows the resulting transition time $T$ for the setpoint transition in Figure 3.1 with the input constraints $u^{*}(t) \in[-0.8,1.6]$ by using the ODEs (3.15) and (3.18), respectively. ${ }^{4}$ For $\delta \rightarrow 1$, the transition time $T$ approaches the time-optimal value $T_{\min } \approx 0.905$ with a bang-bang like feedforward control $u^{*}(t)$. The solid profile of $T(\delta)$ based on the ODEs (3.15) shows a sharp bending, since for $\delta>0.2$ the lower limit $\alpha^{-}$defined by (3.8) crosses the time axis, as illustrated in Figure 3.2a. For this time interval when $0 \notin\left[\alpha^{-}, \alpha^{+}\right]$holds, the normalized value $\hat{\alpha}_{\mathrm{n}}$ in (3.14) is determined by the case (3.14c). The choice $\delta<0.2$ corresponds to a large transition time $T$ with $y^{*(r)}=\hat{\alpha}$ approaching the time axis. Hence, the limits $\alpha^{-}<0<\alpha^{+}$enclose the time axis and $\hat{\alpha}_{\mathrm{n}}$ is determined by (3.14a) or (3.14b) for the whole time interval $t \in[0, T]$. The dashed profile in Figure 3.4 shows that using the ODEs (3.18) instead of (3.15) results in a smoother profile $T(\delta)$ of the transition time $T$ in dependence of $\delta \in(0,1)$.

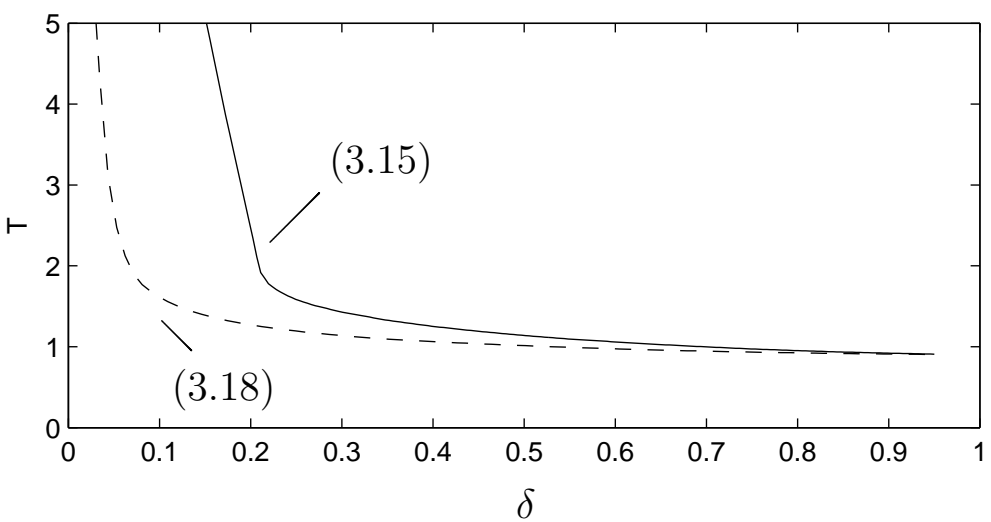

Figure 3.4: Transition time $T$ in dependence of the aggressiveness measure $\delta \in(0,1)$ for the setpoint transition in Figure 3.1 with $u^{*} \in[-0.8,1.6]$.

A drawback of the approach is that the aggressiveness measure $\delta$ does not directly rate the exploitation of the input constraints (3.1). This is mainly due to the fact that the casedependent interpolation function (3.14) is nonlinear. Also the input-output dynamics (2.18) is in general nonlinear which leads to a nonlinear mapping of the input constraints (3.1) to the highest output derivative $y^{*(r)}=\hat{\alpha} \in\left[\alpha^{-}, \alpha^{+}\right]$. Moreover, a rigorous proof of the monotonicity $\partial T / \partial \delta<0$ of the transition time $T(\delta)$ with respect to $\delta$ is difficult to state.

It is also important to mention that the conditioning of the numerical BVP solvability is reduced if the BVPs (3.2)-(3.3) are extended by the ODEs (3.15) or (3.18) and the terminal BC (3.16).

\footnotetext{
${ }^{4}$ The profiles $T(\delta)$ are obtained by calculating $T$ for 10 values of $\delta \in(0,1)$ and using a spline interpolation to smoothen the results.
} 
This is mainly due to the fact that the new states $\omega_{1}(t)$ and $\omega_{2}(t)$ are not separately fixed at both interval bounds $t=0$ and $t=T$ - as in case of $y^{*(i)}(t), i=0,1, \ldots, r-1$ and $\boldsymbol{\eta}^{*}(t)$ in the BVPs (3.2)-(3.3) — but are coupled by the terminal BC in (3.16). Hence, the determination of the transition time $T$ as part of the BVP solution will in general reduce the numerical robustness of the BVP solution and moreover requires a good initial guess of the free transition time $\varepsilon=T$. In contrast to this, if the transition time $T$ is predefined, an initial guess $\boldsymbol{p}=\mathbf{0}$ of the free parameters in the setup function $\Phi(t, \boldsymbol{p})$ is usually sufficient for the numerical solution of the BVPs (3.2)-(3.3).

Nevertheless, the concept of rating the area or volume under the curves $\hat{\alpha}_{\mathrm{n}}$ and $\alpha_{\mathrm{n}}^{+}$by the ODEs (3.15) or (3.18) provides a constructive means to determine $T$ within the feedforward control design while accounting for the systems dynamics (2.1) and input constraints (3.1) in dependence of the aggressiveness measure $\delta \in(0,1)$.

\subsection{Example - Continuous stirred tank reactor}

Continuous stirred tank reactors (CSTR) are popular examples in the control literature due to highly nonlinear dynamics, especially if consecutive and side reactions take place. A benchmark problem for nonlinear control design is the reactor model proposed by Klatt and Engell (1993), also see (Engell and Klatt, 1993; Klatt et al., 1995). The considered CSTR comprises consecutive and parallel reactions and is based on realistic physical considerations and assumptions. Various control concepts are proposed in the literature for controlling the benchmark reactor, e.g. nonlinear predictive control (Chen et al., 1995), flatness-based control (Rothfuss et al., 1996), or inversion-based feedforward control design (Perez et al., 2002; Graichen et al., 2004). A comparison between the flatness-based two-degree-of-freedom control design and nonlinear predictive control can be found in (Utz et al., 2006). In the following, the benchmark reactor is used to illustrate the feedforward control design under input constraints by steering the reactor from an initial to a terminal stationary setpoint under consideration of constraints on the cooling power.

\subsubsection{Reactor model}

The CSTR shown in Figure 3.5 is continuously fed by the (normalized) dilution rate $q$ with the temperature $\vartheta_{\text {in }}$ and the inlet concentration $c_{\text {in }}$. The cooling jacket's temperature $\vartheta_{c}$ is affected by the cooling power $\dot{Q}$ applied to the cooling jacket. The liquid phase inside the reactor is supposed to be of constant volume. The reactions inside the liquid phase are described by the "van de Vusse" reaction scheme (van de Vusse, 1964)

$$
A \longrightarrow B \longrightarrow C, \quad 2 A \longrightarrow D,
$$

comprising the reaction of educt $A$ to the desired product $B$ and the consecutive/parallel reactions to the undesired byproducts $C$ and $D$. The reactor is modeled by the following mass 


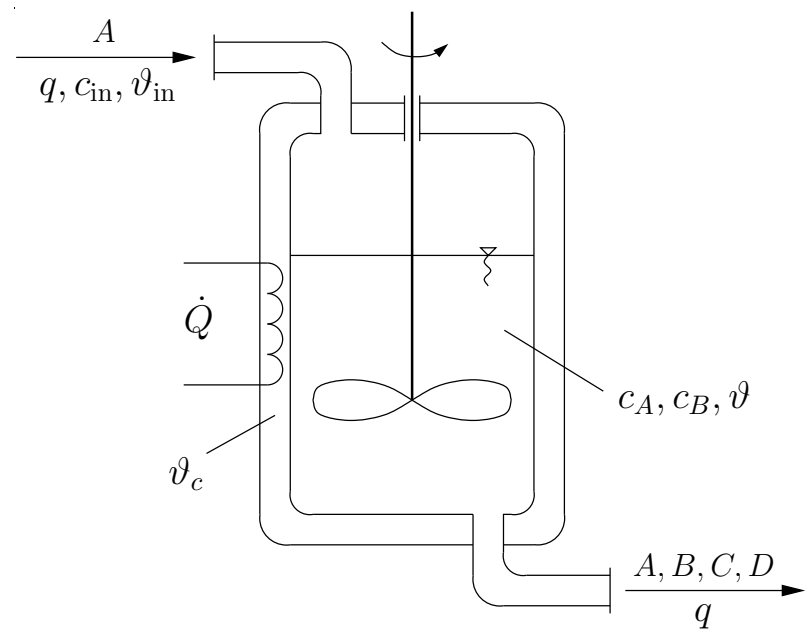

Figure 3.5: Scheme of the CSTR with the "van de Vusse" reaction scheme (3.19).

and energy balances, see e.g. (Klatt et al., 1995; Rothfuss et al., 1996):

$$
\begin{aligned}
\dot{c}_{A} & =-k_{1}(\vartheta) c_{A}-k_{2}(\vartheta) c_{A}^{2}+\left(c_{\mathrm{in}}-c_{A}\right) q \\
\dot{c}_{B} & =k_{1}(\vartheta) c_{A}-k_{1}(\vartheta) c_{B}-c_{B} q \\
\dot{\vartheta} & =h\left(c_{A}, c_{B}, \vartheta\right)+\kappa_{1}\left[\vartheta_{c}-\vartheta\right]+\left[\vartheta_{\text {in }}-\vartheta\right] q \\
\dot{\vartheta}_{c} & =\kappa_{2}\left[\vartheta-\vartheta_{c}\right]+\kappa_{3} \dot{Q}
\end{aligned}
$$

with the concentrations $c_{A}$ and $c_{B}$ of the educt $A$ and product $B$ and the temperatures $\vartheta$ and $\vartheta_{c}$ of the reactor and cooling jacket, respectively. The term $h\left(c_{A}, c_{B}, \vartheta\right)$ in the energy balance $(3.20 \mathrm{c})$ denotes the reaction-induced change in temperature $\vartheta$ and is described by

$$
h\left(c_{A}, c_{B}, \vartheta\right)=-\kappa_{4}\left[k_{1}(\vartheta) c_{A} \Delta H_{A B}+k_{1}(\vartheta) c_{B} \Delta H_{B C}+k_{2}(\vartheta) c_{A}^{2} \Delta H_{A D}\right] .
$$

The reaction rates $k_{1}(\vartheta)$ and $k_{2}(\vartheta)$ depend on the reactor temperature $\vartheta$ and are modeled with the Arrhenius functions ${ }^{5}$

$$
k_{i}(\vartheta)=k_{i 0} \exp \left(\frac{-E_{i}}{\vartheta /{ }^{\circ} \mathrm{C}+273.15}\right), \quad i=1,2 .
$$

All other symbols are constant parameters with the nominal values given in Table 3.1.

The inputs of the reactor are the flow rate $q$ and the cooling power $\dot{Q}$. In the following, the reactor is reduced to a SISO system by treating the dilution rate $q$ as a constant parameter and using the cooling power $\dot{Q}$ as single input $u$. Thereby, the following constraints are defined for $u=\dot{Q}$ (Klatt and Engell, 1993):

$$
u=\dot{Q} \in\left[u^{-}, u^{+}\right] \quad \text { with } \quad u^{-}=-8500 \mathrm{~kJ} / \mathrm{h}, \quad u^{+}=0 \mathrm{~kJ} / \mathrm{h} .
$$

Moreover, the constant dilution rate $q$ possesses the specified bounds $q \in[5,35] \mathrm{h}^{-1}$.

\footnotetext{
${ }^{5}$ Rothfuss et al. (1996) mention that according to (Klatt and Engell, 1993), both steps of the constitutive reaction obey the same mechanism and are thus described by the same reaction rate $k_{1}(\vartheta)$.
} 


\begin{tabular}{lll|lll}
\hline Symbol & Unit & Value & Symbol & Unit & Value \\
\hline$\kappa_{1}$ & $\mathrm{~h}^{-1}$ & 30.828 & $\kappa_{2}$ & $\mathrm{~h}^{-1}$ & 86.688 \\
$\kappa_{3}$ & $\mathrm{~K} / \mathrm{kJ}$ & 0.1 & $\kappa_{4}$ & $\mathrm{~m}^{3} \mathrm{~K} / \mathrm{kJ}$ & $3.522 \cdot 10^{-4}$ \\
$\vartheta_{\text {in }}$ & ${ }^{\circ} \mathrm{C}$ & 104.9 & $c_{\text {in }}$ & $\mathrm{mol} / \mathrm{m}^{3}$ & $5.1 \cdot 10^{3}$ \\
$k_{10}$ & $\mathrm{~h}^{-1}$ & $1.287 \cdot 10^{12}$ & $k_{20}$ & $\mathrm{~m}^{3} /(\mathrm{mol} \mathrm{h})$ & $9.043 \cdot 10^{6}$ \\
$\Delta H_{A B}$ & $\mathrm{~kJ} / \mathrm{mol}$ & 4.2 & $\Delta H_{B C}$ & $\mathrm{~kJ} / \mathrm{mol}$ & -11.0 \\
$\Delta H_{A D}$ & $\mathrm{~kJ} / \mathrm{mol}$ & -41.85 & & & \\
\hline
\end{tabular}

Table 3.1: Parameters of the CSTR model (3.20)-(3.22) taken from (Rothfuss et al., 1996).

The stationary operation points of the reactor model (3.20) are defined in dependence of the desired reactor temperature $\vartheta^{*}$ and the adjusted flow rate $q^{*}$. The remaining stationary states $c_{A}^{*}, c_{B}^{*}, \vartheta_{c}^{*}$ and the input $u^{*}$ are determined by successively solving the stationary equations (3.20a)-(3.20d) with $\dot{c}_{A}^{*}=\dot{c}_{B}^{*}=\dot{\vartheta}^{*}=\dot{\vartheta}_{c}^{*}=0$. The considered setpoint change scenario is to steer the reactor from the stationary state with the temperature $\vartheta^{*}=100{ }^{\circ} \mathrm{C}$ to the stationary setpoint for $\vartheta^{*}=110^{\circ} \mathrm{C}$. Table 3.2 lists the corresponding stationary setpoints of the CSTR for three different values of the normalized flow rate $q$.

\subsubsection{Input-output normal form}

The feedforward control design for the CSTR is based on the input-output coordinates (2.17). An obvious output choice is the reactor temperature

$$
y=\vartheta .
$$

In view of the CSTR model (3.20), differentiating the output (3.24) twice leads to

$$
\begin{aligned}
\dot{y}= & h\left(c_{A}, c_{B}, \vartheta\right)+\kappa_{1}\left[\vartheta_{c}-\vartheta\right]+\left[\vartheta_{\text {in }}-\vartheta\right] q \\
\ddot{y}= & \kappa_{1}\left[\kappa_{2}\left(\vartheta-\vartheta_{c}\right)+\kappa_{3} u\right]+\left[\kappa_{1}\left(\vartheta_{c}-\vartheta\right)+q\left(\vartheta_{\text {in }}-\vartheta\right)+h\left(c_{A}, c_{B}, T\right)\right]\left[\frac{\partial h}{\partial \vartheta}-\kappa_{1}-q\right] \\
& -\left[c_{B} q+\left[c_{B}-c_{A}\right] k_{1}(\vartheta)\right] \frac{\partial h}{\partial c_{B}}-\left[c_{A} k_{1}(\vartheta)+c_{A}^{2} k_{2}(\vartheta)+\left[c_{\text {in }}-c_{A}\right] q\right] \frac{\partial h}{\partial c_{A}} .
\end{aligned}
$$

\begin{tabular}{lcccc}
\hline & $c_{A}^{*}\left[\mathrm{kmol} / \mathrm{m}^{3}\right]$ & $c_{B}^{*}\left[\mathrm{kmol} / \mathrm{m}^{3}\right]$ & $\vartheta_{c}^{*}\left[{ }^{\circ} \mathrm{C}\right]$ & $u^{*}\left[10^{3} \mathrm{~kJ} / \mathrm{h}\right]$ \\
\hline$\vartheta^{*}=100\left[{ }^{\circ} \mathrm{C}\right]$ & & & & \\
$q^{*} \in\{5,8,15\} \mathrm{h}^{-1}$ & $2.02,2.53,3.21$ & $1.07,1.05,0.88$ & $97.1,95.7,93.0$ & $-2.54,-3.76,-6.07$ \\
$\vartheta^{*}=110\left[{ }^{\circ} \mathrm{C}\right]$ & & & & \\
$q^{*} \in\{5,8,15\} \mathrm{h}^{-1}$ & $1.37,1.82,2.50$ & $0.95,1.06,1.07$ & $108.6,108.0,107.0$ & $-1.19,-1.75,-2.61$ \\
\hline
\end{tabular}

Table 3.2: Stationary setpoints of the reactor model (3.20)-(3.22) for the desired reactor temperature $\vartheta^{*}$ and various constant flow rates $q^{*}$. 
Thereby, the input $u$ appears in the second derivative $\ddot{y}$ and the relative degree $r=2$ is welldefined due to the constant parameters $\kappa_{1} \cdot \kappa_{3} \neq 0$, cf. Table 3.1. By choosing the concentrations of educt $A$ and product $B$ as additional coordinates

$$
\boldsymbol{\eta}=\left[\eta_{1}, \eta_{2}\right]^{\top}=\left[c_{A}, c_{B}\right]^{\top}
$$

the cooling jacket temperature $\vartheta_{c}$ follows from (3.25) with the new coordinates $y, \dot{y}$, and $\boldsymbol{\eta}$ :

$$
\vartheta_{c}=y+\left[\dot{y}+q\left[y-\vartheta_{\text {in }}\right]-h\left(\eta_{1}, \eta_{2}, y\right)\right] / \kappa_{1}
$$

The diffeomorphism (2.17) is well-defined since all states $\boldsymbol{x}=\left[c_{A}, c_{B}, \vartheta, \vartheta_{c}\right]^{\top}$ can be expressed by $\left[y, \dot{y}, \boldsymbol{\eta}^{\top}\right]^{\top}=\boldsymbol{\phi}(\boldsymbol{x})$. The input-output normal form (2.18)-(2.19) follows from (3.26) and the material balances (3.20a)-(3.20b) to

$$
\begin{aligned}
\ddot{y} & =\alpha(y, \dot{y}, \boldsymbol{\eta}, u), \\
\dot{\boldsymbol{\eta}} & =\boldsymbol{\beta}(\boldsymbol{\eta}, y) .
\end{aligned}
$$

To steer the reactor from an initial setpoint with the temperature $\vartheta_{0}^{*}$ to a terminal setpoint with $\vartheta_{T}^{*}$ in the finite time interval $t \in[0, T]$ requires to satisfy the following BCs for $y$ and $\boldsymbol{\eta}$ :

$$
\begin{aligned}
y(0) & =\vartheta_{0}^{*}, & y(T) & =\vartheta_{T}^{*}, \\
\boldsymbol{\eta}(0) & =\left[c_{A, 0}^{*}, c_{B, 0}^{*}\right]^{\top}, & \boldsymbol{\eta}(T) & =\left[c_{A, T}^{*}, c_{B, T}^{*}\right]^{\top},
\end{aligned}
$$

whereby the boundary values $c_{A, i}^{*}, c_{B, i}^{*}$, and $\vartheta_{c, i}^{*}, i=0, T$ are taken from Table 3.1 with the desired reactor temperatures $\vartheta_{0}^{*}=100^{\circ} \mathrm{C}$ and $\vartheta_{T}^{*}=110^{\circ} \mathrm{C}$.

Remark 3.3. The stability of the internal dynamics (3.30) can be examined by means of the eigenvalues of its linearization at the setpoints $\boldsymbol{\eta}^{*}=\left[c_{A}^{*}, c_{B}^{*}\right]^{\top}$ in dependence of the temperature $y^{*}=\vartheta^{*}$ and the constant dilution rate $q^{*}$ :

$$
\lambda_{1}=-q^{*}-k_{1}\left(y^{*}\right), \quad \lambda_{2}=-q^{*}-k_{1}\left(y^{*}\right)-2 \eta_{1}^{*} k_{2}\left(y^{*}\right) .
$$

Thereby, $\lambda_{i}<0, i=1,2$ holds due to $q^{*}>0, \eta_{1}^{*}>0$ and the positive Arrhenius functions $k_{i}\left(y^{*}\right)>0$ defined by (3.22). Hence, the internal dynamics is stable for the setpoints $\boldsymbol{\eta}^{*}=$ $\left[c_{A}^{*}, c_{B}^{*}\right]^{\top}$ and the CSTR is minimum-phase with respect to the chosen output $y=\vartheta$ and the single input $u=\dot{Q}$.

However, the CSTR may be nonminimum-phase for different output/input choices, e.g. if the product concentration is the desired output $y=c_{B}$ and the dilution rate is used as input $u=q$ (Engell and Klatt, 1993; Kravaris et al., 1998; Graichen et al., 2004). Moreover, the CSTR operated in MIMO-mode with the outputs $y_{1}=T, y_{2}=c_{B}$ and both inputs $u_{1}=q, u_{2}=\dot{Q}$ may also locally exhibit nonminimum-phase characteristics depending on the respective setpoint (Niemiec and Kravaris, 2003). 


\subsubsection{Feedforward control design for setpoint change}

The inverse of the input-output dynamics (3.29) allows the algebraic calculation of the feedforward control

$$
u^{*}=\alpha^{-1}\left(y^{*}, \dot{y}^{*}, \ddot{y}^{*}, \boldsymbol{\eta}^{*}\right)
$$

in dependence of the output trajectory $y^{*}(t)$ and the internal dynamics state $\boldsymbol{\eta}^{*}(t)$. Thereby, $y^{*}(t)$ and $\boldsymbol{\eta}^{*}(t)$ have to satisfy the BCs (3.31) of the setpoint change. The incorporation of the input constraints (3.23) leads to the BVPs (3.2)-(3.3) with the setup function $\ddot{y}=\hat{\alpha}$ :

$$
\begin{aligned}
& \ddot{y}^{*}=\hat{\alpha}, \quad y^{*}(0)=\vartheta_{0}^{*}, \quad y^{*}(T)=\vartheta_{T}^{*},\left.\quad \dot{y}^{*}\right|_{t=0, T}=0 \\
& \dot{\boldsymbol{\eta}}^{*}=\boldsymbol{\beta}\left(\boldsymbol{\eta}^{*}, y^{*}\right), \quad \boldsymbol{\eta}^{*}(0)=\left[c_{A, 0}^{*}, c_{B, 0}^{*}\right]^{\top}, \quad \boldsymbol{\eta}^{*}(T)=\left[c_{A, T}^{*}, c_{B, T}^{*}\right]^{\top} .
\end{aligned}
$$

The BVPs (3.33)-(3.34) are overdetermined by $2 n=8$ BCs for $n=4$ ODEs. Hence, $n=4$ free parameters $\boldsymbol{p}=\left(p_{1}, \ldots, p_{4}\right)$ are provided in one of the setup functions (3.6) which is used to parameterize $\hat{\alpha}=\Phi(t, \boldsymbol{p})$ if the corresponding feedforward control (3.32)

$$
u_{\Phi}^{*}=\alpha^{-1}\left(y^{*}, \dot{y}^{*}, \Phi(t, \boldsymbol{p}), \boldsymbol{\eta}^{*}\right)
$$

stays inside the constraints $\left[u^{-}, u^{+}\right]$, see (3.23). The sine series (3.6d) is used as parameterization function $\Phi(t, \boldsymbol{p})$ leading to a continuous feedforward control $u^{*}(t)$ due to the homogeneous boundary values $\Phi(0, \boldsymbol{p})=\Phi(T, \boldsymbol{p})=0$.

If the feedforward control (3.35) violates the constraints (3.23), the highest output derivative $\ddot{y}^{*}=\hat{\alpha}$ is replanned according to the case-dependent definition (3.7):

$$
\hat{\alpha}= \begin{cases}\Phi(t, \boldsymbol{p}) & \text { if } u_{\Phi}^{*} \in\left[u^{-}, u^{+}\right] \\ \alpha\left(y^{*}, \dot{y}^{*}, \boldsymbol{\eta}^{*}, u^{-}\right) & \text {if } u_{\Phi}^{*}<u^{-} \\ \alpha\left(y^{*}, \dot{y}^{*}, \boldsymbol{\eta}^{*}, u^{+}\right) & \text {if } u_{\Phi}^{*}>u^{+}\end{cases}
$$

The solution $y^{*}(t)$ and $\boldsymbol{\eta}^{*}(t), t \in[0, T]$ of the BVPs (3.33)-(3.34) with the free parameters $\boldsymbol{p}$ in (3.36) finally yield the feedforward control (3.32) constrained by the limits (3.23).

Remark 3.4. Another important reactor class besides the CSTRs are batch and semi-batch reactors. An industrial benchmark problem for a semi-batch polymerization reactor has been defined by Chylla and Haase (1993). Thereby, the inversion-based feedforward control design can be used to improve the control performance while maintaining the conventional feedback cascade control structure (Graichen et al., 2005b, 2006). A similar flatness-based feedforward control in the spirit of the two-degree-of-freedom control concept is used in (Hagenmeyer and Nohr, 2005) to improve the control performance of an industrial multi-product polymerization reactor.

\subsubsection{Numerical results}

The BVPs (3.33)-(3.34) with (3.35)-(3.36) are solved with the MatLAB function bvp4c. The initial guess for the trajectories $y^{*}\left(t_{k}\right), \eta_{1}^{*}\left(t_{k}\right)$, and $\eta_{2}^{*}\left(t_{k}\right)$ is a linear interpolation between the 
corresponding BCs on a uniform time mesh $t_{k} \in[0, T], k=1, \ldots, 30$. The initial guess of the free parameter is $\boldsymbol{p}=\mathbf{0}$. Figure 3.6 shows the nominal trajectories for the transition between the setpoints in Table 3.2 for the three dilution rates $q^{*} \in\{5,8,15\} \mathrm{h}^{-1}$ and the transition time $T=25 \mathrm{~min}$. For the smaller values of $q^{*}$, the feedforward control $u^{*}(t)$ hits the upper constraint $u^{+}=0 \mathrm{~kJ} / \mathrm{h}$. In these time intervals, the reactor is not cooled in order to raise the temperature $y^{*}(t)=\vartheta^{*}(t)$ as fast as possible. For the largest value of the dilution rate, $q=15 \mathrm{~h}^{-1}$, the feedforward trajectory $u^{*}(t)$ does not touch the constraint $u^{+}$, since the produced reaction heat is large enough to heat the reactor to the desired temperature setpoint $\vartheta_{T}^{*}=110^{\circ} \mathrm{C}$.

Figure 3.6 illustrates how the dilution rate $q^{*}$ affects the reactor dynamics (3.20) and therefore the aggressiveness of the feedforward control $u^{*}(t)$ with respect to the chosen transition time $T=25$ min. For $q^{*}=5 \mathrm{~h}^{-1}$, the input $u^{*}(t)$ stays at the constraint for more than $50 \%$ of the time interval $t \in[0, T]$, whereas $q^{*}=15 \mathrm{~h}^{-1}$ leads to an almost quasi-stationary transition. Hence, in order to calculate the transition time $T$ within the feedforward control design, the BVPs (3.33)-(3.34) are extended by the two ODEs (3.15) and the terminal BC (3.16) depending on the desired aggressiveness measure $\delta \in(0,1)$. The time transformation (3.17) leads to the additional free parameter $\varepsilon=T$. In addition to the above-mentioned initial guesses for $y^{*}\left(t_{k}\right)$, $\eta_{1}^{*}\left(t_{k}\right), \eta_{2}^{*}\left(t_{k}\right)$, and $\boldsymbol{p}=\mathbf{0}$, the states $\omega_{1}\left(t_{k}\right)$ and $\omega_{2}\left(t_{k}\right)$ are set to zero. The reasonable guess for $\varepsilon$ is of importance for the numerical solution of the BVPs with bvp4c. It is set to $\varepsilon=30 \mathrm{~min}$
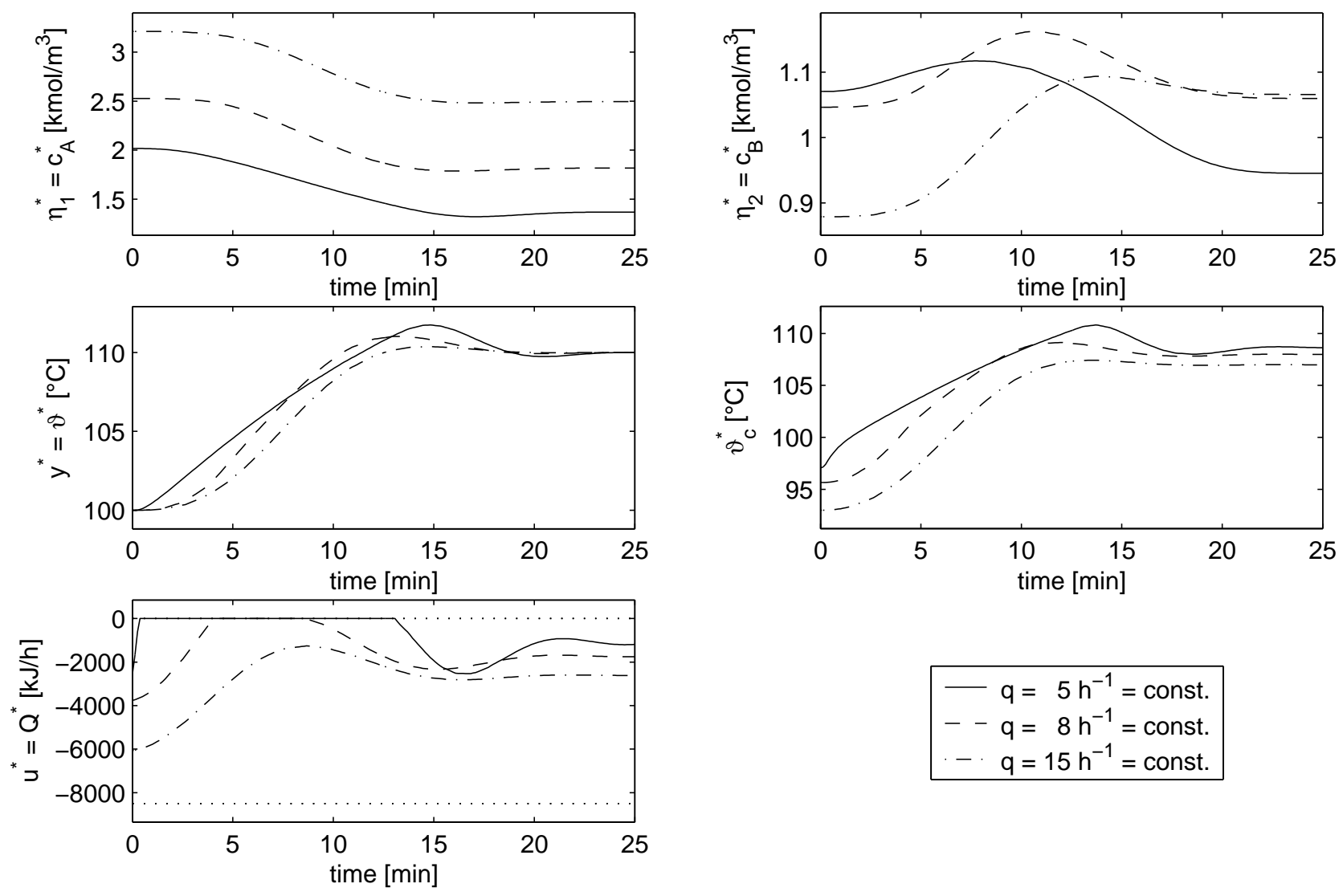

Figure 3.6: Nominal trajectories for the transition of the CSTR (3.20)-(3.23) between the setpoints in Table 3.2 in the transition time $T=25$ min and for three different delusion rates $q^{*}$. 
for small aggressiveness values $\delta<0.1$ and to $\varepsilon=15 \mathrm{~min}$ for $\delta>0.1$. Figure 3.7 shows the calculated transition time $T(\delta)$ in dependence of $\delta$ for three dilution rates $q^{*} \in\{5,8,15\} \mathrm{h}^{-1}{ }^{6}$ As it is also visible in Figure 3.6, the highest dilution rate $q^{*}=15 \mathrm{~h}^{-1}$ leads to the smallest transition time $T$ and approaches a minimum of $T \approx 11 \mathrm{~min}$ for $\delta \rightarrow 1$. The "o"-marks in Figure 3.7 show the aggressiveness ratios $\delta$ for the feedforward trajectories $u^{*}(t)$ in Figure 3.6 with the transition time $T=25 \mathrm{~min}$.

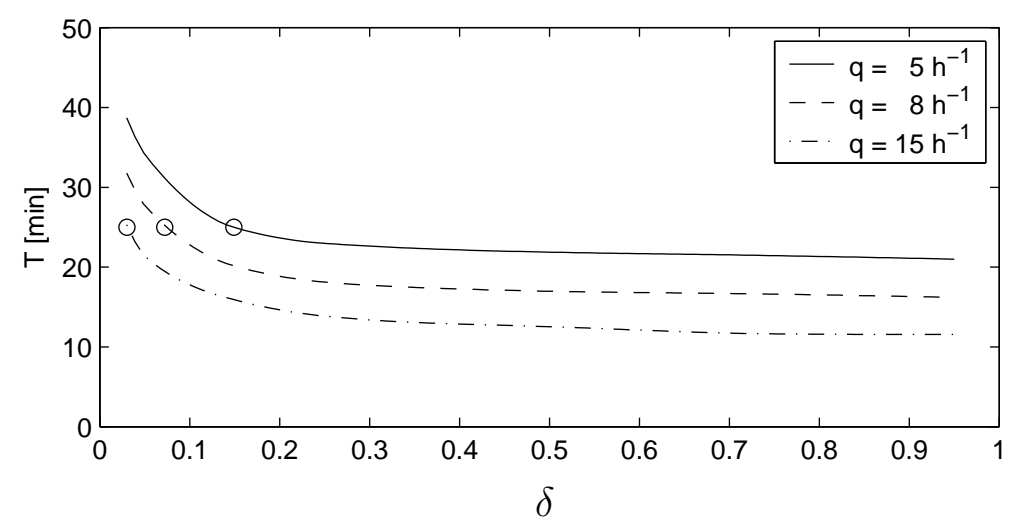

Figure 3.7: Transition time $T$ in dependence of the aggressiveness measure $\delta \in(0,1)$ with the marks "o" corresponding to the setpoint transition in Figure 3.6.

\subsection{Conclusions}

In order to incorporate input constraints within the feedforward control design, the BVP of the internal dynamics is extended by a second BVP for the output $y^{*}$, whereby the highest output derivative $y^{*(r)}$ is either parameterized by a setup function with free parameters or is replanned by means of the input-output dynamics in order to exactly fulfill the input constraints. In this way, the constraints are systematically incorporated in the formulation of the BVPs.

In a further step, an attempt is made to calculate the transition time $T$ within the feedforward control design. In the unconstrained case, $T$ is in general unsuitable as free parameter for the solution of the internal dynamics BVP (see Remark 2.3). In the input-constrained case addressed in this chapter, the aggressiveness of the feedforward control $u^{*}(t) \in\left[u^{-}, u^{+}\right]$can be rated by means of the normalized design parameter $\delta \in(0,1)$. This offers a constructive way to determine the transition time $T$ by augmenting the BVPs of the output and the internal dynamics by two further ODEs and three BCs. The transition time $T$ is treated as additional free parameter by applying a time transformation. However, the coupling of the new states by the terminal BC reduces the numerical solvability of the BVPs and requires a good initial guess of the free transition time $T$.

\footnotetext{
${ }^{6}$ The profiles $T(\delta)$ are obtained by calculating $T$ for 10 values of $\delta \in(0,1)$ and using a spline interpolation to smoothen the profiles.
} 


\section{Chapter 4}

\section{Feedforward control design for nonlinear SISO systems with output constraints}

Besides the consideration of input constraints as addressed in the previous chapter, the feedforward control design often requires to account for constraints on the output $y^{*}(t)$ and its time derivatives. A typical field of application is e.g. motion planning in robotics, where the displacement $y$ may be limited, the velocity $\dot{y}$ has to meet security constraints, or the acceleration $\ddot{y}$ is constrained by a maximum actuator force. ${ }^{1}$ Moreover, constraining the output $y$ is of particular importance for nonminimum-phase systems, where the output trajectory $y^{*}(t)$ typically shows an initial counter-swing behavior, which increases as the transition time $T$ is reduced, see e.g. Figure 2.4. Hence, as schematically shown in Figure 1.2, the feedforward control design is further elaborated in this chapter in order to account for the constraints

$$
y^{*(i)}(t) \in\left[y_{i}^{-}, y_{i}^{+}\right], \quad i=0,1, \ldots, r
$$

on the output trajectory $y^{*}(t)$ and its time derivatives up to order $r$ representing the relative degree of the system (2.1). Thereby, the assumption is made that

$$
y_{i}^{-}<0<y_{i}^{+}, \quad i=1, \ldots, r
$$

holds for the constraints of the output derivatives $y^{*(i)}(t), i=1, \ldots, r$. Usually, the limits $y_{i}^{-}$and $y_{i}^{+}, i=0,1, \ldots, r$ are chosen smaller than the physical constraints in order to leave sufficient control margin for the feedback control $\Sigma_{F B}$ in Figure 1.1.

Similar to the incorporation of the input constraints (3.1) in the previous chapter, the output constraints (4.1) are considered in the formulation of the BVPs for the setpoint transition. This is accomplished by constructing a dynamic system in new coordinates. Its numerical

\footnotetext{
${ }^{1}$ An interesting approach for the online generation of the output trajectory $y^{*}(t)$ with constraints on the first and second output derivative $\dot{y}^{*}(t)$ and $\ddot{y}^{*}(t)$ can be found in (Zanasi et al., 2000).
} 
solution yields the output trajectory $y^{*}(t)$, which directly satisfies the constraints (4.1) due to its particular structure (Graichen and Zeitz, 2006c,b). The new system replaces the BVP (3.2) of the output, in order to directly incorporate the constraints (4.1) in the formulation of the BVPs. Moreover, the constraint $y^{*(r)}(t) \in\left[y_{r}^{-}, y_{r}^{+}\right]$of the highest output derivative can be used to additionally account for the input constraints (3.1). The concept is illustrated for the side-stepping of the triple inverted pendulum with constraints on the cart position $y^{*}$, velocity $\dot{y}^{*}$, and acceleration $\ddot{y}^{*}$.

\subsection{System representation for constrained output}

The basic idea of the presented approach is to systematically incorporate the output constraints (4.1) in a new system representation by introducing saturation functions and successively differentiating the output $y^{*}$ (Graichen and Zeitz, 2006c,b). This section describes how this system representation is derived and how the limits of the saturation functions are determined with respect to the output constraints (4.1).

\subsubsection{Successive incorporation of output constraints}

In order to illustrate the construction of the new system, the concept is firstly illustrated for the output $y^{*}$ and its time derivative $\dot{y}^{*}$, before the general case for $y^{*(i)}, i=1, \ldots, r$ is derived.

\section{Constrained output $y^{*} \in\left[y_{0}^{-}, y_{0}^{+}\right]$}

The constraints for the output $y^{*}$ are considered by introducing the smooth saturation function

$$
y^{*}=\psi_{1}\left(\xi_{1}, \psi_{1}^{ \pm}\right)
$$

with the new coordinate $\xi_{1} \in \mathbb{R}$ and the respective saturation limits $\psi_{1}^{-}$and $\psi_{1}^{+}$, see Figure $4.1 .^{2}$ Thereby, it is assumed that $\psi_{1}^{-}$and $\psi_{1}^{+}$are asymptotic limits and $\psi_{1}\left(\xi_{1}, \psi_{1}^{ \pm}\right)$is strictly monotonically increasing, i.e. $\partial \psi_{1} / \partial \xi_{1}>0$. Obviously, the constraint $y^{*} \in\left[y_{0}^{-}, y_{0}^{+}\right]$is satisfied if the saturation limits are chosen to $\psi_{1}^{-}=y_{0}^{-}$and $\psi_{1}^{+}=y_{0}^{+}$, or written in compact notation:

$$
\psi_{1}^{ \pm}=y_{0}^{ \pm}
$$

Hence, by means of (4.3) and (4.4) the constrained output $y^{*} \in\left[y_{0}^{-}, y_{0}^{+}\right]$is projected onto the new unconstrained coordinate $\xi_{1}$.

\section{Constrained output derivative $\dot{\boldsymbol{y}}^{*} \in\left[\boldsymbol{y}_{1}^{-}, \boldsymbol{y}_{1}^{+}\right]$}

In order to incorporate the constraint for the time derivative $\dot{y}^{*}$ of the output, Equation (4.3) is differentiated

$$
\dot{y}^{*}=\frac{\partial \psi_{1}}{\partial \xi_{1}} \dot{\xi}_{1}
$$

\footnotetext{
${ }^{2}$ To simplify matters, the notation $(\cdot)^{ \pm}$is used in the following to address both symbols $(\cdot)^{-}$and $(\cdot)^{+}$.
} 


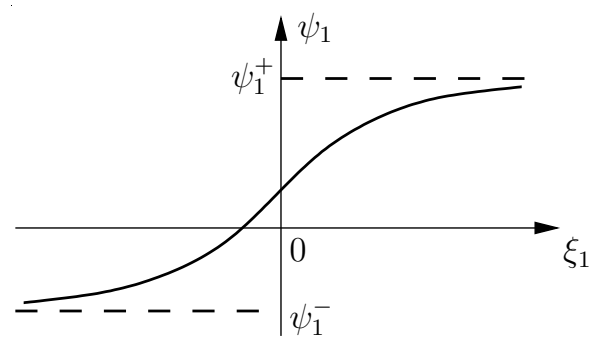

Figure 4.1: Smooth saturation function $\psi_{1}\left(\xi_{1}, \psi_{1}^{ \pm}\right)$with the limits $\psi_{1}^{-}, \psi_{1}^{+}$and the new coordinate $\xi_{1}$.

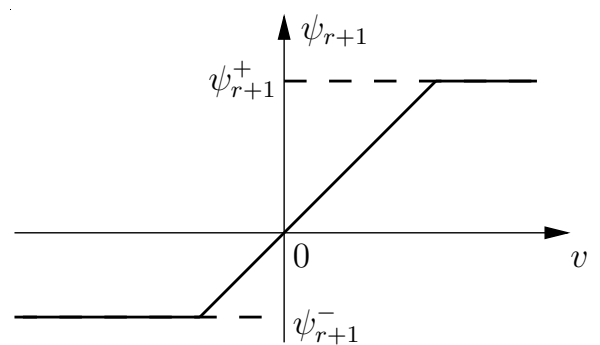

Figure 4.2: Ramp-shaped saturation function $\psi_{r+1}\left(v, \psi_{r+1}^{ \pm}\right)$with the limits $\psi_{r+1}^{-}, \psi_{r+1}^{+}$ and the new input $v$.

and a second saturation function

$$
\dot{\xi}_{1}=\psi_{2}\left(\xi_{2}, \psi_{2}^{ \pm}\right)
$$

is introduced for $\dot{\xi}_{1}$ with the new coordinate $\xi_{2} \in \mathbb{R}$. The saturation limits $\psi_{2}^{ \pm}$have to be chosen appropriately to satisfy the constraint $\dot{y}^{*} \in\left[y_{1}^{-}, y_{1}^{+}\right]$. In view of (4.5) and (4.6) and with the above assumption $\partial \psi_{1} / \partial \xi_{1}>0$, the inequality $-y_{1}^{-} \leq \dot{y}^{*} \leq y_{1}^{+}$can be formulated as

$$
y_{1}^{-}\left[\frac{\partial \psi_{1}}{\partial \xi_{1}}\right]^{-1} \leq \psi_{2}\left(\xi_{2}, \psi_{2}^{ \pm}\right) \leq y_{1}^{+}\left[\frac{\partial \psi_{1}}{\partial \xi_{1}}\right]^{-1}
$$

where the left and right sides of the inequality represent the limits

$$
\psi_{2}^{ \pm}\left(\xi_{1}\right)=y_{1}^{ \pm}\left[\frac{\partial \psi_{1}}{\partial \xi_{1}}\right]^{-1}
$$

for the saturation function $\psi_{2}\left(\xi_{2}, \psi_{2}^{ \pm}\right)$. Hence, the limits $\psi_{2}^{ \pm}=\psi_{2}^{ \pm}\left(\xi_{1}\right)$ not only depend on $y_{1}^{ \pm}$ but also on the previously introduced coordinate $\xi_{1}$ in order to satisfy the output constraint $\dot{y}^{*} \in\left[y_{1}^{-}, y_{1}^{+}\right]$.

General case $y^{*(i)} \in\left[y_{i}^{-}, y_{i}^{+}\right], i=1, \ldots, r$

The Equations (4.5)-(4.7) illustrate the idea, how the constraint (4.1) of the $i$-th output derivative $y^{*(i)}$ is considered. After differentiating $\dot{y}^{*}$ in (4.5), a new saturation function $\dot{\xi}_{2}=\psi_{3}\left(\xi_{3}, \psi_{3}^{ \pm}\right)$is introduced in dependence of $\xi_{3}$. Thereby, the limits $\psi_{3}^{ \pm}=\psi_{3}^{ \pm}\left(\boldsymbol{\xi}_{2}\right)$ will depend on the coordinates $\boldsymbol{\xi}_{2}=\left[\xi_{1}, \xi_{2}\right]^{\top}$ in order to meet the output constraint $\ddot{y}^{*} \in\left[y_{2}^{-}, y_{2}^{+}\right]$.

The $r$-th differentiation $y^{*(r)}$ of the output leads to the final saturation

$$
\dot{\xi}_{r}=\psi_{r+1}\left(v, \psi_{r+1}^{ \pm}\right)
$$

with a new input $v$ (see Section 4.2.3). The limits $\psi_{r+1}^{ \pm}=\psi_{r+1}^{ \pm}\left(\boldsymbol{\xi}_{r}\right)$ depend on the previously introduced coordinates $\boldsymbol{\xi}_{r}=\left[\xi_{1}, \ldots, \xi_{r}\right]^{\top}$. Since no further output differentiation is required, a ramp-shaped saturation function is sufficient for $\psi_{r+1}\left(v, \psi_{r+1}^{ \pm}\right)$, see Figure 4.2. 
Incorporating the output constraints (4.1) in the above-mentioned way leads to a dynamical system for the coordinates (or respectively states) $\xi_{i}, i=1, \ldots, r$ with the triangular structure

$$
\left.\begin{array}{rl}
y^{*}= & \psi_{1}\left(\xi_{1}, \psi_{1}^{ \pm}\right) \\
\dot{\xi}_{1}= & \psi_{2}\left(\xi_{2}, \psi_{2}^{ \pm}\left(\xi_{1}\right)\right) \\
\dot{\xi}_{2}= & \psi_{3}\left(\xi_{3}, \psi_{3}^{ \pm}\left(\boldsymbol{\xi}_{2}\right)\right) \\
\vdots & \vdots \\
\dot{\xi}_{r}= & \psi_{r+1}\left(v, \psi_{r+1}^{ \pm}\left(\boldsymbol{\xi}_{r}\right)\right)
\end{array}\right\} \quad \dot{\boldsymbol{\xi}}_{r}=\boldsymbol{\psi}_{r+1}\left(\boldsymbol{\xi}_{r}, v\right)
$$

and the new input $v$. The vectors are given by

$$
\boldsymbol{\xi}_{0}=\emptyset, \quad \boldsymbol{\xi}_{i}=\left[\xi_{1}, \ldots, \xi_{i}\right]^{\top}, \quad i=1, \ldots, r, \quad \boldsymbol{\psi}_{r+1}=\left[\psi_{2}, \ldots, \psi_{r+1}\right]^{\top} .
$$

As a result, the new system (4.9) yields the output trajectory $y^{*}(t)$, which satisfies the constraints (4.1). Figure 4.3 shows the signal diagram of the system (4.9), whereby the triangular structure is apparent in the feedback of the states $\xi_{i}$ to the saturation functions $\psi_{i}^{ \pm}\left(\boldsymbol{\xi}_{i-1}\right), i=2, \ldots, r+1$.

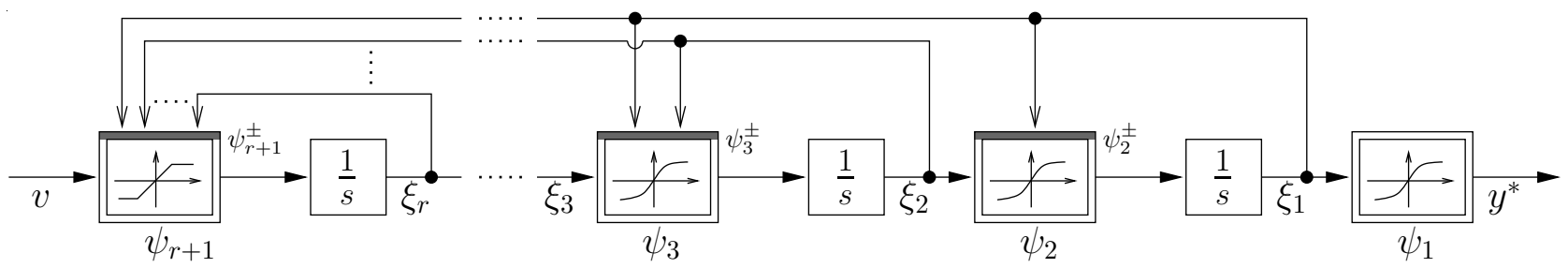

Figure 4.3: Signal diagram of the dynamical system (4.9) with the states $\xi_{i}, i=1, \ldots, r$, the new input $v$, the output $y=\psi_{1}\left(\xi_{1}, \psi_{1}^{ \pm}\right)$, and the saturation functions $\psi_{i}, i=2, \ldots, r+1$.

\subsubsection{Choice of saturation functions}

As mentioned in the previous section, the first $r$ saturation functions $\psi_{i}\left(\xi_{i}, \psi_{i}^{ \pm}\left(\boldsymbol{\xi}_{i-1}\right)\right), i=$ $1, \ldots, r$ are assumed to be strictly monotonic, such that the limits $\psi_{i}^{ \pm}\left(\boldsymbol{\xi}_{i-1}\right), i=1, \ldots, r$ are only reached asymptotically, i.e.

$$
\frac{\partial \psi_{i}}{\partial \xi_{i}}>0, \quad \psi_{i}^{-}<\psi_{i}<\psi_{i}^{+}, \quad i=1, \ldots, r
$$

An appropriate setup is e.g.

$$
\psi_{i}\left(\xi_{i}, \psi_{i}^{ \pm}\right)=\psi_{i}^{+}-\frac{\psi_{i}^{+}-\psi_{i}^{-}}{1+\exp \left[m \xi_{i}\right]}
$$

whereby for $i>1$ the asymptotic limits $\psi_{i}^{ \pm}=\psi_{i}^{ \pm}\left(\boldsymbol{\xi}_{i-1}\right)$ depend on the states $\boldsymbol{\xi}_{i-1}$ defined in (4.10). Figure 4.1 shows this saturation function schematically for $i=1$. The parameter $m$ 
adjusts the slope at the position $\xi_{i}=0$ and is chosen to

$$
m=\frac{4}{\psi_{i}^{+}-\psi_{i}^{-}}
$$

corresponding to the slope $\partial \psi_{i} / \partial \xi_{i}=1$ at $\xi_{i}=0$.

Since the monotonicity condition (4.11) is not required for the last function $\psi_{r+1}\left(v, \psi_{r+1}^{ \pm}\left(\boldsymbol{\xi}_{r}\right)\right)$, the case-dependent definition of a ramp-shaped saturation function

$$
\psi_{r+1}\left(v, \psi_{r+1}^{ \pm}\left(\boldsymbol{\xi}_{r}\right)\right)= \begin{cases}v & \text { if } v \in\left[\psi_{r+1}^{-}\left(\boldsymbol{\xi}_{r}\right), \psi_{r+1}^{+}\left(\boldsymbol{\xi}_{r}\right)\right] \\ \psi_{r+1}^{-}\left(\boldsymbol{\xi}_{r}\right) & \text { if } v<\psi_{r+1}^{-}\left(\boldsymbol{\xi}_{r}\right) \\ \psi_{r+1}^{+}\left(\boldsymbol{\xi}_{r}\right) & \text { if } v>\psi_{r+1}^{+}\left(\boldsymbol{\xi}_{r}\right)\end{cases}
$$

is used, see Figure 4.2.

\subsubsection{Calculation of saturation limits}

Equation (4.7) illustrates for the first output derivative $\dot{y}^{*}$ how the saturation limits $\psi_{2}^{ \pm}\left(\xi_{1}\right)$ are to be determined to meet the constraint $\dot{y}^{*} \in\left[y_{1}^{-}, y_{1}^{+}\right]$. In order to incorporate the constraints (4.1) in the general case $y^{*(i)}$, the $i$-th output derivative $y^{*(i)}$ of the output is derived according to the following lemma.

Lemma 4.1. The $i$-th time derivative $y^{*(i)}, i=1, \ldots, r$ of the output $y^{*}=\psi_{1}\left(\xi_{1}, \psi_{1}^{ \pm}\right)$in $(4.3)$ can be written in the form

$$
y^{*(i)}=L_{\boldsymbol{\psi}_{r+1}}^{i} \psi_{1}\left(\xi_{1}, \psi_{1}^{ \pm}\right)=\gamma_{i}\left(\boldsymbol{\xi}_{i}\right)+\prod_{j=1}^{i} \frac{\partial \psi_{j}}{\partial \xi_{j}} \cdot \psi_{i+1}\left(\cdot, \psi_{i+1}^{ \pm}\left(\boldsymbol{\xi}_{i}\right)\right)
$$

with $L_{\boldsymbol{\psi}_{r+1}}$ denoting the Lie derivative along the vector field $\boldsymbol{\psi}_{r+1}$ in (4.9). The argument (·) in $\psi_{i+1}\left(\cdot, \psi_{i+1}^{ \pm}\left(\boldsymbol{\xi}_{i}\right)\right)$ is given by $\xi_{i+1}$ or $v$ for $i=r$, respectively.

Proof. The first time derivative $\dot{y}^{*}$ (i.e. $i=1$ ) in Equation (4.5) satisfies the form (4.14) with $\gamma_{1}\left(\xi_{1}\right)=0$. For the higher derivatives $y^{*(i)}, i>1$, Equation (4.14) can be proved by induction. The induction is started for $i=2$ by differentiating (4.5):

$$
\ddot{y}^{*}=\underbrace{\frac{\partial^{2} \psi_{1}}{\partial \xi_{1}^{2}} \psi_{2}^{2}+\frac{\partial \psi_{1}}{\partial \xi_{1}} \frac{\partial \psi_{2}}{\partial \xi_{1}} \psi_{2}}_{=\gamma_{2}\left(\boldsymbol{\xi}_{2}\right)}+\frac{\partial \psi_{1}}{\partial \xi_{1}} \frac{\partial \psi_{2}}{\partial \xi_{2}} \psi_{3}\left(\xi_{3}, \psi_{3}^{ \pm}\left(\boldsymbol{\xi}_{2}\right)\right) .
$$

The inductive hypothesis states

$$
y^{*(i-1)} \stackrel{!}{=} \gamma_{i-1}\left(\boldsymbol{\xi}_{i-1}\right)+\prod_{j=1}^{i-1} \frac{\partial \psi_{j}}{\partial \xi_{j}} \cdot \psi_{i}\left(\xi_{i}, \psi_{i}^{ \pm}\left(\boldsymbol{\xi}_{i-1}\right)\right) .
$$


The inductive steps follows by differentiating $y^{*(i-1)}$, i.e.

$$
y^{*(i)}=\frac{\mathrm{d}}{\mathrm{d} t} y^{*(i-1)}=\frac{\partial}{\partial \boldsymbol{\xi}_{r}}\left[\gamma_{i-1}\left(\boldsymbol{\xi}_{i-1}\right)+\prod_{j=1}^{i-1} \frac{\partial \psi_{j}}{\partial \xi_{j}} \cdot \psi_{i}\left(\xi_{i}, \psi_{i}^{ \pm}\left(\boldsymbol{\xi}_{i-1}\right)\right)\right] \boldsymbol{\psi}_{r+1} .
$$

Due to the lower triangular structure of (4.9), this expression can be written with the reducedorder vector fields $\boldsymbol{\psi}_{i}=\left[\psi_{2}, \ldots, \psi_{i}\right]^{\top}$ and $\boldsymbol{\xi}_{i-1}$ given in (4.10):

$$
y^{*(i)}=\underbrace{\frac{\partial}{\partial \boldsymbol{\xi}_{i-1}}\left[\gamma_{i-1}\left(\boldsymbol{\xi}_{i-1}\right)+\prod_{j=1}^{i-1} \frac{\partial \psi_{j}}{\partial \xi_{j}} \cdot \psi_{i}\left(\xi_{i}, \psi_{i}^{ \pm}\left(\boldsymbol{\xi}_{i-1}\right)\right)\right]}_{=\gamma_{i}\left(\boldsymbol{\xi}_{i}\right)} \boldsymbol{\psi}_{i}+\underbrace{\frac{\partial}{\partial \xi_{i}}\left[\prod_{j=1}^{i-1} \frac{\partial \psi_{j}}{\partial \xi_{j}} \psi_{i}\right]}_{=\prod_{j=1}^{i} \frac{\partial \psi_{j}}{\partial \xi_{j}}} \psi_{i+1}\left(\cdot, \psi_{i+1}^{ \pm}\left(\boldsymbol{\xi}_{i}\right)\right) .
$$

Hence, the differentiation of $y^{*(i-1)}$ leads to the same structure as $(4.15)$, which proves the inductive hypothesis and Equation (4.14).

The nonlinear term $\gamma_{i}\left(\boldsymbol{\xi}_{i}\right)$ in (4.14) structurally divides the equation for $y^{*(i)}$ in two parts. As mentioned in the proof of Lemma 4.1, $\gamma_{1}\left(\xi_{1}\right)=0$ holds for $i=1$. For $i>1, \gamma_{i}\left(\boldsymbol{\xi}_{i}\right)$ is derived by applying the chain and product rule in course of the output differentiation.

Equation (4.14) defines the relation between the output derivative $y^{*(i)}$ and the respective saturation function $\psi_{i+1}\left(\cdot, \psi_{i+1}^{ \pm}\left(\boldsymbol{\xi}_{i}\right)\right)$. Thereby, the following inequality holds for the output constraints $y^{*(i)} \in\left[y_{i}^{-}, y_{i}^{+}\right]$:

$$
y_{i}^{-} \leq \gamma_{i}\left(\boldsymbol{\xi}_{i}\right)+\prod_{j=1}^{i} \frac{\partial \psi_{j}}{\partial \xi_{j}} \cdot \psi_{i+1}\left(\cdot, \psi_{i+1}^{ \pm}\left(\boldsymbol{\xi}_{i}\right)\right) \leq y_{i}^{+} .
$$

Due to Condition (4.11), the term $\gamma_{i}\left(\boldsymbol{\xi}_{i}\right)$ can be subtracted on both sides of the inequality and dividing by $\prod_{j=1}^{i} \partial \psi_{j} / \partial \xi_{j}>0$ yields

$$
\frac{y_{i}^{-}-\gamma_{i}\left(\boldsymbol{\xi}_{i}\right)}{\prod_{j=1}^{i} \frac{\partial \psi_{j}}{\partial \xi_{j}}} \leq \psi_{i+1}\left(\cdot, \psi_{i+1}^{ \pm}\left(\boldsymbol{\xi}_{i}\right)\right) \leq \frac{y_{i}^{+}-\gamma_{i}\left(\boldsymbol{\xi}_{i}\right)}{\prod_{j=1}^{i} \frac{\partial \psi_{j}}{\partial \xi_{j}}} .
$$

Hence, the limits of the saturation function $\psi_{i+1}\left(\cdot, \psi_{i+1}^{ \pm}\left(\boldsymbol{\xi}_{i}\right)\right)$ follow from the above equation in dependence of the constraints $y_{i}^{-}$and $y_{i}^{+}$(the first case (4.4) is repeated for the sake of completeness):

$$
\psi_{1}^{ \pm}=y_{0}^{ \pm}, \quad \psi_{i+1}^{ \pm}\left(\boldsymbol{\xi}_{i}\right)=\frac{y_{i}^{ \pm}-\gamma_{i}\left(\boldsymbol{\xi}_{i}\right)}{\prod_{j=1}^{i} \frac{\partial \psi_{j}}{\partial \xi_{j}}}, \quad i=1, \ldots, r
$$

As a result, the system (4.9) is constructed by successively differentiating the output $y^{*}$ to obtain the nonlinear terms $\gamma_{i}\left(\boldsymbol{\xi}_{i}\right)$ in $(4.14)$, which are required to determine the saturation limits (4.17). The underlying analytic calculations can be performed with computer-algebrasystems like MATHEMATiCA or MAPLE. 
Remark 4.1. If no constraints are defined for the output $y^{*}$ or one of its output derivatives, i.e. $y^{*(j)} \in\left[y_{j}^{-}, y_{j}^{+}\right]$with $y_{j}^{ \pm} \rightarrow \pm \infty$ holds for a certain $j \in\{0,1, \ldots, r\}$, then the saturation limits (4.17) are also given by $\psi_{j+1}^{ \pm}\left(\boldsymbol{\xi}_{j}\right) \rightarrow \pm \infty$. Hence, the respective saturation function in (4.12) or $(4.13)$ can be replaced by $\psi_{j+1}\left(\xi_{j+1}, \psi_{j+1}\left(\boldsymbol{\xi}_{j}\right)\right)=\xi_{j+1}$ or $\psi_{r+1}\left(v, \psi_{r+1}\left(\boldsymbol{\xi}_{r}\right)\right)=v$ for $j=r$, respectively.

Remark 4.2. The first $r$ saturation functions $\psi_{i}\left(\xi_{i}, \psi_{i}^{ \pm}\left(\boldsymbol{\xi}_{i-1}\right)\right), i=1, \ldots, r$ in (4.12) are assumed strictly monotonically increasing, in order to simplify the derivation of the saturation limits (4.17). However, the construction of the dynamic system (4.9) for the constrained output trajectory $y^{*}(t)$ is generally valid for non-asymptotic saturation functions such that the output constraints (4.1) are exactly fulfilled, see Appendix $C$.

\subsection{Boundary value problem for constrained output}

The output constraints (4.1) are incorporated within the feedforward control design by substituting the output BVP (3.2) by the new system representation (4.9) ${ }^{3}$

$$
\begin{array}{lll}
\dot{\boldsymbol{\xi}}_{r}=\boldsymbol{\psi}_{r+1}\left(\boldsymbol{\xi}_{r}, v\right), & \boldsymbol{\xi}_{r}(0)=\boldsymbol{\xi}_{r, 0}, & \boldsymbol{\xi}_{r}(T)=\boldsymbol{\xi}_{r, T}, \\
\dot{\boldsymbol{\eta}}^{*}=\overline{\boldsymbol{\beta}}\left(\boldsymbol{\eta}^{*}, y^{*}, \ldots, y^{*(r)}\right), & \boldsymbol{\eta}^{*}(0)=\boldsymbol{\eta}_{0}^{*}, & \boldsymbol{\eta}^{*}(T)=\boldsymbol{\eta}_{T}^{*}
\end{array}
$$

The output variables $y^{*(i)}, i=0,1, \ldots, r$ appearing in the internal dynamics (4.19) are determined by the relations (4.3) and (4.14) in dependence of the state $\boldsymbol{\xi}_{r}$. This leads to a one-side coupling of the BVPs (4.18)-(4.19). Moreover, the BCs of the output $y^{*}$ in (3.2) must be converted to the boundary values $\boldsymbol{\xi}_{r, 0}$ and $\boldsymbol{\xi}_{r, T}$ of the new states $\boldsymbol{\xi}_{r}=\left[\xi_{1}, \ldots, \xi_{r}\right]^{\top}$.

\subsubsection{Calculation of boundary conditions}

The boundary values of the first coordinate $\xi_{1}$ are obtained by inverting the saturation function $\psi_{1}\left(\xi_{1}, \psi_{1}^{ \pm}\right)$in $(4.3)$ :

$$
\xi_{1,0}=\psi_{1}^{-1}\left(y_{0}^{*}, \psi_{1}^{ \pm}\right), \quad \xi_{1, T}=\psi_{1}^{-1}\left(y_{T}^{*}, \psi_{1}^{ \pm}\right)
$$

Clearly, the stationary values $y_{0}^{*}$ and $y_{T}^{*}$ must lie inside the constraints (4.4), since otherwise $\psi_{1}\left(\xi_{1}, \psi_{1}^{ \pm}\right)$is not invertible with respect to $\xi_{1}$. The remaining boundary values $\xi_{i, 0}$ and $\xi_{i, T}, i=$ $2, \ldots, r$ can be determined with the homogeneous BCs $y^{*(i)}(0)=y^{*(i)}(T)=0, i=1, \ldots, r-1$ and the following lemma.

\footnotetext{
${ }^{3}$ The asterisk $\left({ }^{*}\right)$ is omitted for the new states $\boldsymbol{\xi}_{r}$ as they represent "artificial" coordinates compared to the "physical" output $y^{*}$.
} 
Lemma 4.2. If the output (4.3) approaches a stationary value $y^{*}=$ const. with the vanishing derivatives $y^{*(i)}=0, i=1, \ldots, r$, then the system (4.9) is described by the stationary equations

$$
\left.\begin{array}{l}
0=\psi_{2}\left(\xi_{2}, \psi_{2}^{ \pm}\left(\xi_{1}\right)\right) \\
0=\psi_{3}\left(\xi_{3}, \psi_{3}^{ \pm}\left(\boldsymbol{\xi}_{2}\right)\right) \\
\vdots \quad \vdots \\
0=\psi_{r+1}\left(v, \psi_{r+1}^{ \pm}\left(\boldsymbol{\xi}_{r}\right)\right)
\end{array}\right\} \quad \mathbf{0}=\boldsymbol{\psi}_{r+1}\left(\boldsymbol{\xi}_{r}, v\right)
$$

Moreover, the respective saturation limits $\psi_{i}^{-}\left(\boldsymbol{\xi}_{i-1}\right)$ and $\psi_{i}^{+}\left(\boldsymbol{\xi}_{i-1}\right)$ satisfy the inequality

$$
\psi_{i}^{-}\left(\boldsymbol{\xi}_{i-1}\right)<0<\psi_{i}^{+}\left(\boldsymbol{\xi}_{i-1}\right), \quad i=2, \ldots, r+1
$$

Proof. Equation (4.21) can again be proved by induction. The inductive start $i=1$ for the first output derivative $\dot{y}^{*}$ is based on the equations (4.5) and (4.6). Since $\partial \psi_{1} / \partial \xi_{1}>0$ holds, $\dot{y}^{*}=0$ leads to $\psi_{2}\left(\xi_{2}, \psi_{2}^{ \pm}\left(\xi_{1}\right)\right)=0$. The inductive hypothesis for $i-1$ assumes that for vanishing derivatives $y^{*(k)}=0, k=1, \ldots, i-1$, the saturation functions $\boldsymbol{\psi}_{i}=\left[\psi_{2}, \ldots, \psi_{i}\right]^{\top}$ are given by $\boldsymbol{\psi}_{i}=\mathbf{0}$. In order to prove the hypothesis, the inductive step for $y^{*(i)}=0$ is conducted by differentiating $y^{*(i-1)}$, which yields Equation (4.16) in the proof of Lemma 4.1. Thereby, $\prod_{j=1}^{i} \partial \psi_{j} / \partial \xi_{j}>0$ holds due to the monotonicity condition (4.11). For $y^{*(i)}=0$ and with the inductive hypothesis $\boldsymbol{\psi}_{i}=\mathbf{0}$, Equation (4.16) directly leads to $\psi_{i+1}\left(\cdot, \psi_{i+1}\left(\boldsymbol{\xi}_{i}\right)\right)=0$ which proves (4.21).

The proof of Equation (4.22) relies on the fact that for the stationary output $y^{*}$ with $y^{*(i)}=$ $0, i=1, \ldots, r-1$, the nonlinear terms $\gamma_{i}\left(\boldsymbol{\xi}_{i}\right), i=1, \ldots, r$ in (4.14) are also zero. This follows from (4.16) with $\boldsymbol{\psi}_{i}=0$ as the result of the first part of this proof. Hence, using $\gamma_{i}\left(\boldsymbol{\xi}_{i}\right)=0$ in (4.17) and an index shift leads to the simplified saturation limits

$$
\psi_{i}^{ \pm}\left(\boldsymbol{\xi}_{i-1}\right)=\frac{y_{i-1}^{ \pm}}{\prod_{j=1}^{i-1} \frac{\partial \psi_{j}}{\partial \xi_{j}}}, \quad i=2, \ldots, r+1
$$

The assumption (4.2) and $\prod_{j=1}^{i} \partial \psi_{j} / \partial \xi_{j}>0$ ensure that the saturation limits (4.23) enclose zero, which proves the inequality (4.22).

Based on the results of Lemma 4.2 , the boundary values $\xi_{i, 0}$ and $\xi_{i, T}, i=2, \ldots, r$ are calculated by inverting the stationary equations $(4.21)$ with respect to $\xi_{i}$ :

$$
\xi_{i, 0}=\psi_{i}^{-1}\left(0, \psi_{i}^{ \pm}\left(\boldsymbol{\xi}_{i-1,0}\right)\right), \quad \xi_{i, T}=\psi_{i}^{-1}\left(0, \psi_{i}^{ \pm}\left(\boldsymbol{\xi}_{i-1, T}\right)\right), \quad i=2, \ldots, r .
$$

Hence, $\xi_{i, 0}$ and $\xi_{i, T}$ depend on the previously determined values $\boldsymbol{\xi}_{i-1,0}$ and $\boldsymbol{\xi}_{i-1, T}$, whereby the inequality (4.22) ensures that the inverse functions $\psi_{i}^{-1}$ exist. 


\subsubsection{Special case - symmetric constraints}

The expressions (4.24) can be simplified if the constraints (4.1) for the output derivatives are symmetric, i.e.

$$
y_{i}^{-}=-y_{i}^{+}, \quad i=1, \ldots, r-1 .
$$

In this case, the saturation limits $(4.23)$ are also symmetric, i.e. $\left|\psi_{i+1}^{-}\left(\boldsymbol{\xi}_{i}\right)\right|=\left|\psi_{i+1}^{+}\left(\boldsymbol{\xi}_{i}\right)\right|$, such that the saturation functions (4.21) pass through the origin $0=\psi_{i}\left(0, \psi_{i}^{ \pm}\left(\boldsymbol{\xi}_{i-1}\right)\right)$, also see (4.12). Hence, the boundary values $\xi_{i, 0}$ and $\xi_{i, T}$ in (4.24) simplify to

$$
\xi_{i, 0}=\xi_{i, T}=0, \quad i=2, \ldots, r .
$$

The calculation of the BCs for the BVP (4.18) thus reduces to the solution of (4.20) for $\xi_{1,0}$ and $\xi_{1, T}$, whereas the further $\mathrm{BCs}$ for the states $\xi_{i}, i=2, \ldots, r$ are homogeneous.

\subsubsection{Setup function with free parameters}

The solutions $\boldsymbol{\xi}_{r}(t)=\left[\xi_{1}(t), \ldots, \xi_{r}(t)\right]^{\top}$ and $\boldsymbol{\eta}^{*}(t), t \in[0, T]$ of the two BVPs (4.18)-(4.19) depend on the choice of the new input $v$ with respect to the following points:

(i) In analogy to point (i) in Section 3.1, the highest output derivative $y^{*(r)}$ has to satisfy the BCs in (2.20) for $\rho=r$ if the feedforward control $u^{*}(t)$ in (2.26) shall be continuous at the bounds $t=0$ and $t=T$. As shown in Equation (4.21) of Lemma 4.2, the BCs $y^{*(r)}(0)=y^{*(r)}(T)=0$ lead to the last stationary equation $0=\psi_{r+1}\left(v, \psi_{r+1}^{ \pm}\left(\boldsymbol{\xi}_{r}\right)\right)$ of the system (4.9). Since the ramp-shaped saturation function $\psi_{r+1}\left(v, \psi_{r+1}\left(\boldsymbol{\xi}_{r}\right)\right)$ passes through the origin (see Figure 4.2, Equation (4.13), and (4.22) for $i=r+1$ ), the new input $v$ has to satisfy the homogeneous BCs

$$
v(0)=0, \quad v(T)=0
$$

if continuity of the feedforward control $u^{*}(t)$ is desired.

(ii) The two BVPs (4.18)-(4.19) are overdetermined by $2 n$ BCs for $n$ ODEs. Similar to the points (ii)-(iii) in Section 3.1, $n$ free parameters $\boldsymbol{p}=\left(p_{1}, \ldots, p_{n}\right)$ are provided in a setup function for the new input

$$
v=\Phi(t, \boldsymbol{p}),
$$

whereby one of the polynomial or trigonometric functions in (3.6) can be employed to parameterize $\Phi(t, \boldsymbol{p})$ depending on the desired continuity property of the feedforward control $u^{*}(t)$.

The solution of the two BVPs (4.18)-(4.19) with the free parameters $\boldsymbol{p}$ in (4.28) comprises the trajectories $\boldsymbol{\xi}_{r}(t)$ and $\boldsymbol{\eta}^{*}(t), t \in[0, T]$. The output trajectory $y^{*}(t)$ and its time derivatives $y^{*(i)}(t), i=1, \ldots, r$ are algebraically calculated with Equation (4.3) and (4.14), whereby the construction of the system (4.9) (also see (4.18), respectively) ensures that the output constraints (4.1) are satisfied. The feedforward trajectory $u^{*}(t)$ finally follows from (2.26). 
Example 4.1 (Example 3.1 continued). Due to the instability of the internal dynamics (3.10b) of the linear SISO system $(n=2, r=1)$, the output trajectory $y^{*}(t)$ shows an undershoot at the beginning of the transition in Figure 3.1. In order to reduce the undershoot of $y^{*}(t)$ and to additionally constrain the output derivative $\dot{y}^{*}(t)$, the output BVP (3.10a) is replaced by the new BVP (4.18) for the coordinate $\xi$ while maintaining the internal dynamics (3.10b):

$$
\begin{array}{rlrl}
\dot{\xi} & =\psi_{2}\left(v, \psi_{2}^{ \pm}(\xi)\right), & \xi(0)=\xi_{0}, & \xi(T)=\xi_{T}, \\
\dot{\eta}^{*}=-a_{0} y^{*}-\frac{b_{0}}{b_{1}}\left(a_{1} y^{*}+\dot{y}^{*}-\eta^{*}\right)=\bar{\beta}\left(\eta^{*}, y^{*}, \dot{y}^{*}\right) & \eta^{*}(0)=\eta_{0}^{*}, & \eta^{*}(T)=\eta_{T}^{*} .
\end{array}
$$

The output functions

$$
y^{*}=\psi_{1}\left(\xi, \psi_{1}^{ \pm}\right), \quad \dot{y}^{*}=\frac{\partial \psi_{1}}{\partial \xi} \psi_{2}\left(v, \psi_{2}^{ \pm}(\xi)\right)
$$

are used in the internal dynamics (4.29b) to express $y^{*}$ and $\dot{y}^{*}$ in terms of the coordinate $\xi$ and the new input $v$. The saturation functions $\psi_{1}\left(\xi_{1}, \psi_{1}^{ \pm}\right)$and $\psi_{2}\left(v, \psi_{2}^{ \pm}(\xi)\right)$ are defined by (4.12) and (4.13) with the respective limits $\psi_{1}^{ \pm}$and $\psi_{2}^{ \pm}(\xi)$ in (4.4) and (4.7). The boundary values $\xi_{0}$ and $\xi_{T}$ in (4.29a) are determined according to (4.20).

The BVPs (4.29) are overdetermined by 4 BCs for 2 first-order ODEs. Hence, 2 free parameters $\boldsymbol{p}=\left(p_{1}, p_{2}\right)$ are provided in the new input $v=\Phi(t, \boldsymbol{p})$ with the sine series $(3.6 \mathrm{~d})$ (alternatively the polynomial (3.6c) may be used). The BVPs are solved with bvp4c and a linear interpolation between the respective $B C$ s serving as initial guess for the trajectories $\xi\left(t_{k}\right)$ and $\eta^{*}\left(t_{k}\right)$ on the
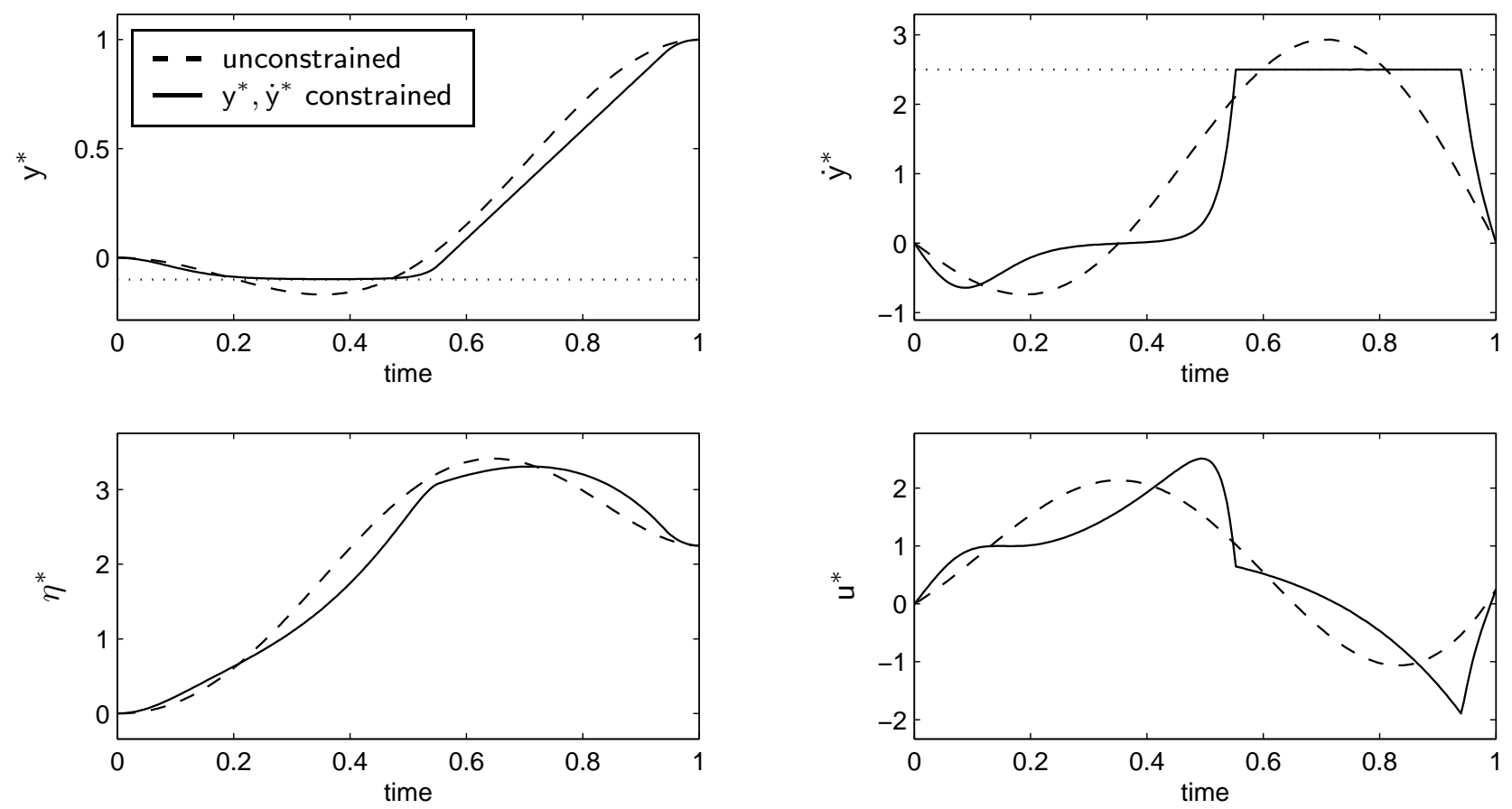

Figure 4.4: Setpoint transition $y_{0}^{*}=0 \rightarrow y_{T}^{*}=1$ with $T=1$ of the linear SISO system (1.1) based on the solution of the BVPs (4.29) with the parameters (2.15) and output constraints $y^{*} \in[-0.1,1.1]$ and $\dot{y}^{*} \in[-2.5,2.5]$. 
uniform time mesh $t_{k} \in[0, T], k=1, \ldots, 30$. The initial guess of the parameters is $p_{1}=p_{2}=0$. Figure 4.4 shows the output trajectories (4.30), the internal dynamics state $\eta^{*}(t)$, and the feedforward control $u^{*}(t), t \in[0, T]$ in (2.34) for the output constraints $y^{*} \in[-0.1,1.1]$ and $\dot{y}^{*} \in[-2.5,2.5]$ compared to the unconstrained case by setting $y_{0}^{ \pm}$and $y_{1}^{ \pm}$to sufficiently large values. The initial undershoot of $y^{*}(t)$ is clearly reduced and $y^{*}(t)$ almost touches the constraint $y_{0}^{-}=-0.1$ in the approximate time interval $t \in[0.2,0.5]$ before it approaches the final setpoint $y_{T}^{*}=1$ with the maximum velocity $y_{1}^{+}=2.5$. Hereby, the minimal distance of $y^{*}(t)$ to the constraint $y_{0}^{-}$is negligible, although the saturation function $y=\psi_{1}\left(\xi, \psi_{1}^{ \pm}\right)$reaches the respective limits $\psi_{1}^{-}=y_{0}^{-}$only asymptotically.

\subsection{Incorporation of input constraints}

In Section 3.1, the input constraints (3.1) are mapped to constraints on the highest output derivative $y^{*(r)}$ via the definition of $\hat{\alpha}$ in (3.7). In case of the output-constrained system (4.9), the last saturation function $\psi_{r+1}\left(v, \psi_{r+1}^{ \pm}\left(\boldsymbol{\xi}_{r}\right)\right)$ is replaced by a new function $\widehat{\psi}_{r+1}$ for the highest output derivative in (4.14), i.e.

$$
y^{*(r)}=\gamma_{r}\left(\boldsymbol{\xi}_{r}\right)+\prod_{j=1}^{r} \frac{\partial \psi_{j}}{\partial \xi_{j}} \cdot \widehat{\psi}_{r+1}
$$

Thereby, the function $\widehat{\psi}_{r+1}$ has to be defined according to (3.7) in order to account for the input constraints $u^{*} \in\left[u^{-}, u^{+}\right]$. Hence, the input $v$ is used to parameterize $\widehat{\psi}_{r+1}=v$ if the corresponding feedforward control $(2.26)$

$$
u_{v}^{*}=\alpha^{-1}\left(y^{*}, \ldots, y^{*(r-1)}, y_{v}^{*(r)}, \boldsymbol{\eta}^{*}\right) \quad \text { with } \quad y_{v}^{*(r)}=\gamma_{r}\left(\boldsymbol{\xi}_{r}\right)+\prod_{j=1}^{r} \frac{\partial \psi_{j}}{\partial \xi_{j}} \cdot v
$$

stays inside the constraints $\left[u^{-}, u^{+}\right]$. The respective output derivative $y_{v}^{*(r)}$ follows from $(4.31)$ with $\widehat{\psi}_{r+1}=v$. On the other hand, if $u_{v}^{*}$ violates one of the constraints $\left[u^{-}, u^{+}\right]$, then according to $(3.7), y^{*(r)}$ in (4.31) must be set to $y^{*(r)} \stackrel{!}{=} \alpha\left(y^{*}, \ldots, y^{*(r-1)}, \boldsymbol{\eta}^{*}, u^{ \pm}\right)$such that the feedforward control (2.26) stays at the constraint $u^{-}$or $u^{+}$. This yields the case-dependent definition ${ }^{4}$

$$
\widehat{\psi}_{r+1}\left(\boldsymbol{\xi}_{r}, \boldsymbol{\eta}^{*}, v\right)= \begin{cases}v & \text { if } u_{v}^{*} \in\left[u^{-}, u^{+}\right] \\ \frac{\alpha\left(y^{*}, \ldots, y^{*(r-1)}, \boldsymbol{\eta}^{*}, u^{ \pm}\right)-\gamma_{r}\left(\boldsymbol{\xi}_{r}\right)}{\prod_{j=1}^{r} \frac{\partial \psi_{j}}{\partial \xi_{j}}} & \text { if } u_{v}^{*} \gtrless u^{ \pm}\end{cases}
$$

\footnotetext{
${ }^{4}$ Note that the BCs (4.27) still hold for the new input $v$ if continuity of the feedforward control $u^{*}(t)$ at $t=0$ and $t=T$ is desired. It follows from Lemma 4.2 that $\left.\widehat{\psi}_{r+1}\right|_{0, T}=0$ must hold for the new function $\widehat{\psi}_{r+1}$. This directly leads to $\left.v\right|_{0, T}=0$, since the stationary input $u_{v}^{*}=\alpha^{-1}\left(y_{0, T}^{*}, 0, \ldots, 0, \boldsymbol{\eta}_{0, T}^{*}\right)=u_{0, T}^{*}$ in $(4.32)$ must lie within the constraints $u_{v}^{*} \in\left[u^{-}, u^{+}\right]$.
} 
whereby $\widehat{\psi}_{r+1}$ depends on the input $v$ as well as on the states $\boldsymbol{\eta}^{*}$ and $\boldsymbol{\xi}_{r}$ due to the algebraic output relations (4.3) and (4.14). By using the new function (4.33) instead of the previous saturation function $\psi_{r+1}\left(v, \psi_{r+1}^{ \pm}\left(\boldsymbol{\xi}_{r}\right)\right)$, the BVPs (4.18)-(4.19) can be written as

$$
\begin{array}{rlrl}
\dot{\boldsymbol{\xi}}_{r} & =\widehat{\boldsymbol{\psi}}_{r+1}\left(\boldsymbol{\xi}_{r}, \boldsymbol{\eta}^{*}, v\right), & \boldsymbol{\xi}_{r}(0)=\boldsymbol{\xi}_{r, 0}, & \boldsymbol{\xi}_{r}(T)=\boldsymbol{\xi}_{r, T}, \\
\dot{\boldsymbol{\eta}}^{*}=\overline{\boldsymbol{\beta}}\left(\boldsymbol{\eta}^{*}, y^{*}, \ldots, y^{*(r)}\right), & \boldsymbol{\eta}^{*}(0)=\boldsymbol{\eta}_{0}^{*}, & \boldsymbol{\eta}^{*}(T)=\boldsymbol{\eta}_{T}^{*}
\end{array}
$$

Thereby, the internal dynamics state $\boldsymbol{\eta}^{*}$ now affects the BVP (4.34) due to the new vector field $\widehat{\boldsymbol{\psi}}_{r+1}=\left[\psi_{2}, \ldots, \psi_{r}, \widehat{\psi}_{r+1}\right]^{\top}$, in contrast to the previous one-side coupling of the BVPs (4.18)-(4.19) if no input constraints are considered.

By means of (4.33), the input constraints (3.1) are projected to the constraints (3.8) on the highest output derivative $y^{*(r)}=\left[y_{r}^{-}, y_{r}^{+}\right]$, i.e.

$$
y_{r}^{ \pm}=\alpha^{ \pm}\left(y^{*}, \dot{y}^{*}, \ldots, y^{*(r-1)}, \boldsymbol{\eta}^{*}\right)
$$

while the first $r$ output constraints $y^{*(i)} \in\left[y_{i}^{-}, y_{i}^{+}\right], i=0,1, \ldots, r-1$ are still accounted for.

Remark 4.3. An alternative to replacing the saturation function $\psi_{r+1}\left(v, \psi_{r+1}^{ \pm}\left(\boldsymbol{\xi}_{r}\right)\right)$ by $(4.33)$ is to directly use the projected output constraints (4.36) to calculate the saturation limits (4.17) for $\psi_{r+1}\left(v, \psi_{r+1}^{ \pm}\left(\boldsymbol{\xi}_{r}, \boldsymbol{\eta}^{*}\right)\right)$ according to the definition (4.13) (Graichen and Zeitz, 2006c). However, the new function (4.33) checks the resulting feedforward control $u_{v}^{*}$ for $\widehat{\psi}_{r+1}=v$, which corresponds to the incorporation of the input constraints by (3.7). Moreover, the definition of (4.33) can be extended to the MIMO case in a straightforward manner, see Chapter 5.

A critical issue is that the $r$ output constraints $y^{*(i)} \in\left[y_{i}^{-}, y_{i}^{+}\right], i=0,1, \ldots, r-1$ and the projected input constraint (4.36) for $y^{*(r)} \in\left[y_{r}^{-}, y_{r}^{+}\right]$may cause conflicts. If the output $y^{*}$ or one of its time derivatives approaches the respective constraints, i.e. $y^{*(k)} \rightarrow y_{k}^{ \pm}$holds for a certain $k \in\{0,1, \ldots, r-1\}$, then the higher output derivatives $y^{*(i)} \rightarrow 0, i=k+1, \ldots, r$ tend to zero. Hence, the constraints (4.36) for $y^{*(r)}$ have to satisfy the inequality

$$
\alpha^{-}\left(y^{*}, \dot{y}, \ldots, y^{*(r-1)}, \boldsymbol{\eta}^{*}\right)<0<\alpha^{+}\left(y^{*}, \dot{y}, \ldots, y^{*(r-1)}, \boldsymbol{\eta}^{*}\right)
$$

to ensure that $y^{*(r)} \rightarrow 0$ lies inside the constraints $\left[y_{r}^{-}, y_{r}^{+}\right]{ }^{5}$ Otherwise, if one of the constraints (4.36) crosses zero, i.e. $y_{r}^{-}=\alpha^{-}>0$ or $y_{r}^{+}=\alpha^{+}<0$, this leads to conflicts if at the same time $y^{*(r)} \rightarrow 0$ is enforced due to an active constraint $y^{*(k)} \rightarrow y_{k}^{ \pm}, k<r$ in one of the lower output derivatives.

The problems that occur in this situation can be explained in the following way: if the $k_{-}^{-}$ th output variable $y^{*(k)}$ approaches one of the constraints $y_{k}^{ \pm}$, then also the corresponding saturation function $\psi_{k+1}\left(\xi_{k+1}, \psi_{k+1}\left(\boldsymbol{\xi}_{k}\right)\right)$ in (4.3) or (4.14) approaches one of its saturation limits $\psi_{k+1}^{ \pm}$, which leads to $\partial \psi_{k+1} / \partial \xi_{k+1} \rightarrow 0$. Hence, the function $\widehat{\psi}_{r+1}$ in (4.33) has almost

\footnotetext{
${ }^{5}$ This also justifies the assumption (4.2) which is necessary to ensure that the output constraints (4.1) do not contradict each other.
} 
no influence on the highest output derivative $y^{*(r)}$, since the product $\prod_{j=1}^{r} \partial \psi_{j} / \partial \xi_{j}$ in $(4.31)$ tends to zero. As a result, $\widehat{\psi}_{r+1}$ must assume large values in order to steer $y^{*(r)} \in\left[y_{r}^{-}, y_{r}^{+}\right]$away from zero and to remain inside the constraints (4.36) if either $y_{r}^{-}=\alpha^{-}>0$ or $y_{r}^{+}=\alpha^{+}<0$ holds. These peaks in $\widehat{\psi}_{r+1}$ might lead to singularities in the BVPs $(4.34)-(4.35)$ such that their solvability becomes ill-conditioned or impossible. The next example illustrates this point.

Example 4.2 (Example 4.1 continued). In order to incorporate input constraints $u^{*} \in\left[u^{-}, u^{+}\right]$ in the BVPs (4.29) of the linear SISO example, the saturation function $\psi_{2}\left(v, \psi_{2}^{ \pm}\left(\xi_{1}\right)\right)$ is replaced by $\widehat{\psi}_{2}\left(\xi, \eta^{*}, v\right)$ according to (4.33):

$$
\begin{aligned}
& \dot{\xi}=\widehat{\psi}_{2}\left(\xi, \eta^{*}, v\right) \quad \xi(0)=\xi_{0}, \quad \xi(T)=\xi_{T}, \\
& \dot{\eta}^{*}=-a_{0} y^{*}-\frac{b_{0}}{b_{1}}\left(a_{1} y^{*}+\dot{y}^{*}-\eta^{*}\right)=\bar{\beta}\left(\eta^{*}, y^{*}, \dot{y}^{*}\right) \quad \eta^{*}(0)=\eta_{0}^{*}, \quad \eta^{*}(T)=\eta_{T}^{*}
\end{aligned}
$$

with the respective output functions (4.30)

$$
y^{*}=\psi_{1}\left(\xi, \psi_{1}^{ \pm}\right), \quad \dot{y}^{*}=\frac{\partial \psi_{1}}{\partial \xi} \widehat{\psi}_{2}\left(\xi, \eta^{*}, v\right) .
$$

The input-output dynamics and the feedforward control of the linear SISO example which are required for the definition (4.32)-(4.33) of $\widehat{\psi}_{2}\left(\xi, \eta^{*}, v\right)$ follow from (2.22) and (2.34). Moreover, the function $\widehat{\psi}_{2}\left(\xi, \eta^{*}, v\right)$ transforms the input constraints $u^{*} \in\left[u^{-}, u^{+}\right]$to the limits (4.36) for $\dot{y}^{*} \in\left[y_{1}^{-}, y_{1}^{+}\right]$, i.e.

$$
\begin{aligned}
& y_{1}^{-}=\alpha^{-}\left(y^{*}, \eta^{*}\right)=-a_{1} y^{*}+\eta^{*}-b_{1} u^{+}, \\
& y_{1}^{+}=\alpha^{+}\left(y^{*}, \eta^{*}\right)=-a_{1} y^{*}+\eta^{*}-b_{1} u^{-}, \quad b_{1}>0 .
\end{aligned}
$$

in dependence of $\eta^{*}$ and the new coordinate $\xi$ due to the algebraic relation between $y^{*}$ and $\xi$ in (4.39). The saturation function $\psi_{1}\left(\xi_{1}, \psi_{1}^{ \pm}\right)$, the new input $v$, as well as the boundary values $\xi_{0}$ and $\xi_{T}$ are taken from Example 4.1.

Figure 4.5 shows the simulation results for the same scenario as in Figure 4.4 and with the same initial guess for the bvp4c-solution of the BVPs (4.38). At the beginning of the transition, the output trajectory $y^{*}(t)$ almost touches the asymptotic constraint $y_{0}^{-}$such that both the gradient $\partial \psi_{1} / \partial \xi$ of the saturation function $y^{*}=\psi_{1}\left(\xi, \psi_{1}^{ \pm}\right)$and the output derivative $\dot{y}^{*}(t)$ approach zero, cf. Figure 4.5. However, during this time interval of approximately $t \in[0.2,0.4], \eta^{*}(t)$ increases and causes the lower constraint $y_{1}^{-}=\alpha^{-}\left(y^{*}, \eta^{*}, u^{+}\right)$for $\dot{y}^{*}(t)$ to cross zero. Hence, the second saturation function $\widehat{\psi}_{2}\left(\xi, \eta^{*}, v\right)$ shows an impulse-like peak at $t \approx 0.4$ in order to steer $\dot{y}^{*}=\frac{\partial \psi_{1}}{\partial \xi} \widehat{\psi}_{2}\left(\xi, \eta^{*}, v\right)$ along the lower limit $y_{1}^{-}=\alpha^{-}\left(y^{*}, \eta^{*}, u^{+}\right)>0$. Due to the large amplitude of $\widehat{\psi}_{2}\left(\xi, \eta^{*}, v\right)$ as the right-hand side of $(4.38 \mathrm{a})$, the coordinate $\xi$ increases significantly such that the output $y^{*}=\psi_{1}\left(\xi, \psi_{1}^{ \pm}\right)$finally leaves the saturation vicinity and approaches the setpoint $y_{T}^{*}=1$. The impulse-like peak of $\widehat{\psi}_{2}\left(\xi, \eta^{*}, v\right)$ in the ODE (4.38a) reduces the conditioning of the numerical BVP solution thus leading to a higher number of iterations before the solver bvp4c is converged. The solution was obtained by using the sine series (3.6d) as setup function $v=\Phi(t, \boldsymbol{p})$ and without providing analytic Jacobians. However, if e.g. the polynomial (3.6c) is used instead, the analytic Jacobians are essential for the solvability of the BVPs. 

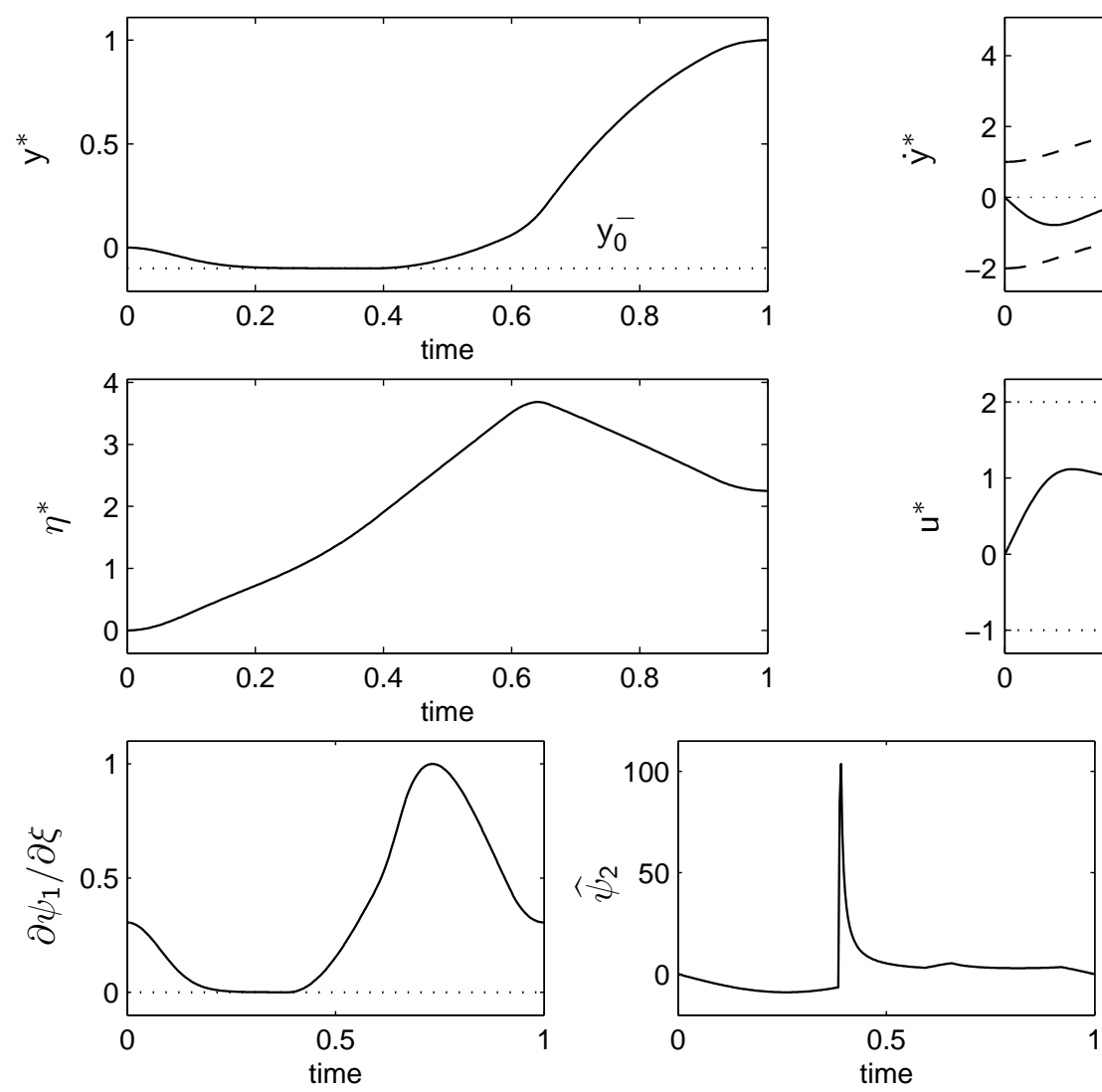
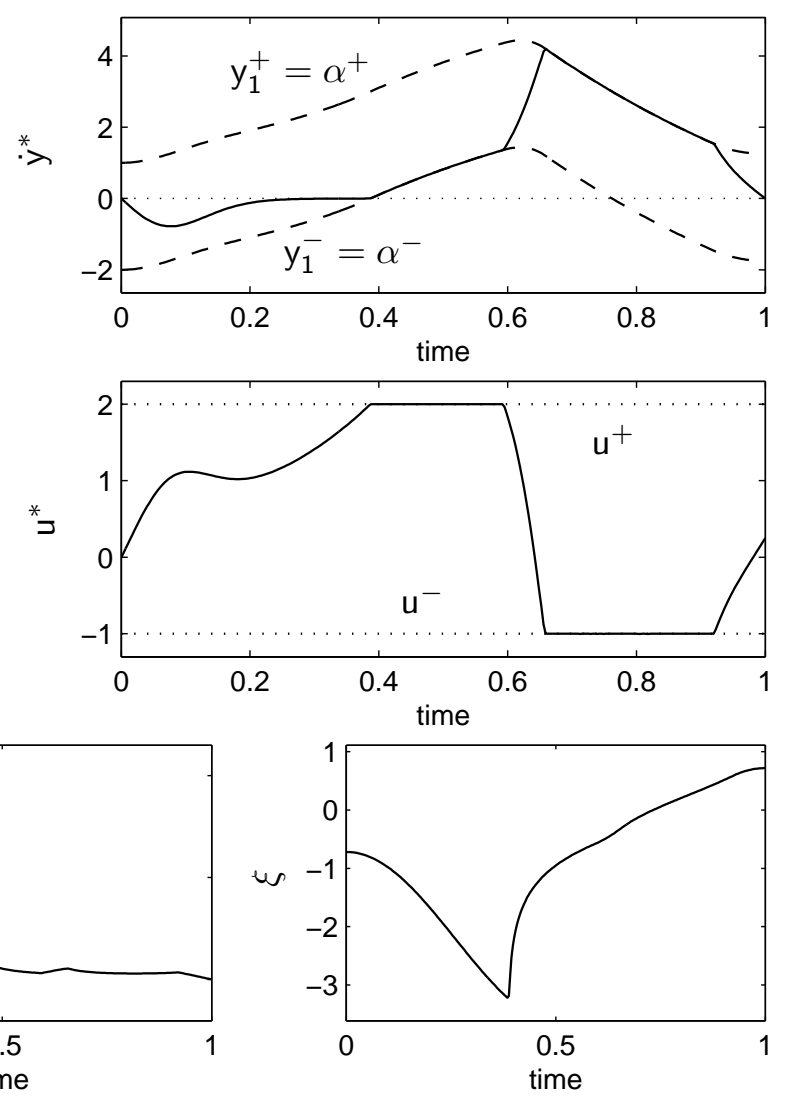

Figure 4.5: Setpoint transition $y_{0}^{*}=0 \rightarrow y_{T}^{*}=1$ with $T=1$ for the linear SISO system (1.1) based on the solution of the BVPs (4.29) with the parameters (2.15) and constraints on the output $y^{*} \in[-0.1,1.1]$ and input $u^{*} \in[-1,2]$.

\subsection{Example - Side-stepping of the triple inverted pendulum on a cart}

The triple pendulum on a cart is an extension of the double pendulum considered in Section 2.4. In contrast to the popularity of single and double pendulums in the control literature (see references in Section 2.4), only few contributions address the triple pendulum. This is mainly due the fact that already the stabilization in the upward position and especially its experimental realization is a difficult problem due to the high instability and sensitivity of the triple pendulum with respect to disturbances, joint friction, and measurement noise. For instance, Medrano-Cerda (1997) and Tsachouridis and Medrano-Cerda (1999) design time-discrete $H_{\infty}$ and LQ controllers in combination with a reduced order observer to stabilize the triple inverted pendulum on an inclined rail, whereas Eltohamy and Kuo (1998) employ a nonlinear optimization technique to design a linear state feedback control to stabilize the triple pendulum. All of these contributions provide simulations and experimental results.

An even more challenging control task is the side-stepping maneuver, i.e. the lateral transition between two unstable upward equilibria. With respect to the two-degree-of-freedom control 
scheme in Figure 1.1, the side-stepping problem can be seen as an extension of the stabilization problem, since the feedforward control $\Sigma_{F F}$ provides the nominal input trajectory for the side-stepping, while the feedback control $\Sigma_{F B}$ stabilizes the triple pendulum in the upward position. In this section, the feedforward control design under output constraints is illustrated for the side-stepping of the triple inverted pendulum with constraints on the cart position, velocity, and acceleration (Graichen et al., 2005c; Graichen and Zeitz, 2006b). The feedback control $\Sigma_{F B}$ is based on an LQ design with the pendulum model linearized around the upward unstable equilibrium. The side-stepping is experimentally validated with the same pendulum construction as described in Section 2.4 for the swing-up of the double pendulum. Nevertheless, some points and explanations are repeated in the following in order to keep the chapter self-contained.

\subsubsection{Problem statement}

The triple pendulum on a cart in Figure 4.6 corresponds to the double pendulum with an additional third pendulum link (also see Figure 2.5). The three links are described by the angles $\eta_{i}, i=1,2,3$ to the vertical direction and the mechanical parameters of the links in Table 4.1. Thereby, the inner and outer pendulum arms, i.e. $i=1,3$, are also used for the double pendulum and are therefore described by the same parameters as in Table 2.1. The cart position serves as output $y$ and is subject to the physical constraints (2.41).

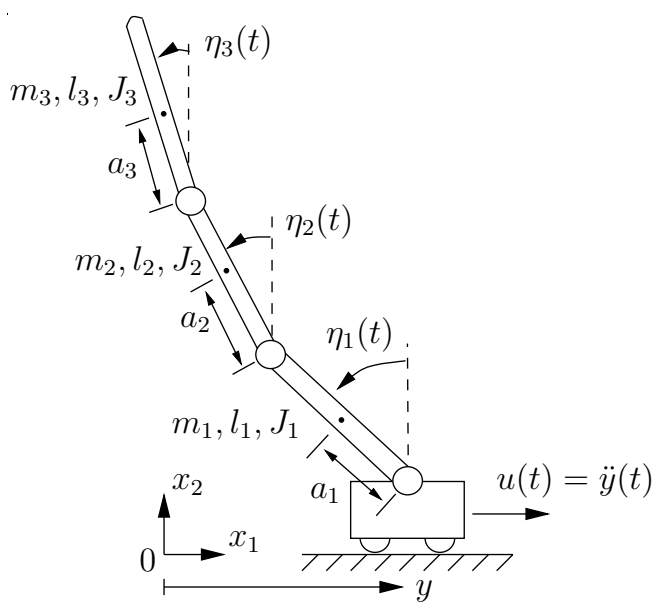

Figure 4.6: Schematic of the triple pendulum with the mechanical parameters in Table 4.1.

\begin{tabular}{lccc} 
Pendulum link & $\begin{array}{l}\text { inner } \\
i=1\end{array}$ & $\begin{array}{l}\text { middle } \\
i=2\end{array}$ & $\begin{array}{l}\text { outer } \\
i=3\end{array}$ \\
\hline length $l_{i}[\mathrm{~m}]$ & 0.323 & 0.419 & 0.480 \\
dist. to center of gravity $a_{i}[\mathrm{~m}]$ & 0.215 & 0.273 & 0.223 \\
mass $m_{i}[\mathrm{~kg}]$ & 0.853 & 0.908 & 0.510 \\
moment of inertia $J_{i}\left[\mathrm{~N} \mathrm{~m} \mathrm{~s}^{2}\right]$ & 0.013 & 0.024 & 0.019 \\
friction constant $d_{i}[\mathrm{Nm} \mathrm{s}]$ & 0.005 & 0.005 & 0.005 \\
\hline
\end{tabular}

Table 4.1: Mechanical parameters of the triple pendulum.

\subsubsection{Equations of motion}

The equations of motion are derived by the Lagrangian method in analogy to the double pendulum. The absolute position $\boldsymbol{x}^{i}=\left[x_{1}^{i}, x_{2}^{i}\right]^{\top}, i=1,2,3$ of the center of mass of the three 
links is given by

$$
\begin{aligned}
& \text { Link 1: } \boldsymbol{x}^{1}=\left[\begin{array}{c}
y-a_{1} \sin \eta_{1} \\
a_{1} \cos \eta_{1}
\end{array}\right], \quad \text { Link 2: } \boldsymbol{x}^{2}=\left[\begin{array}{c}
y-l_{1} \sin \eta_{1}-a_{2} \sin \eta_{2} \\
l_{1} \cos \eta_{1}+a_{2} \cos \eta_{2}
\end{array}\right], \\
& \text { Link 3: } \boldsymbol{x}^{3}=\left[\begin{array}{c}
y-l_{1} \sin \eta_{1}-l_{2} \sin \eta_{2}-a_{3} \sin \eta_{3} \\
l_{1} \cos \eta_{1}+l_{2} \cos \eta_{2}+a_{3} \cos \eta_{3}
\end{array}\right] .
\end{aligned}
$$

For the kinetic and potential energy holds

$$
T=\frac{1}{2} m_{c} \dot{y}^{2}+\frac{1}{2} \sum_{i=1}^{3}\left[m_{i}\left|\dot{\boldsymbol{x}}^{i}\right|^{2}+J_{i} \dot{\eta}_{i}^{2}\right], \quad V=\sum_{i=1}^{3} m_{i} g x_{2}^{i} .
$$

The Lagrangian $L=T-V$ yields the equations of motion

$$
\frac{\mathrm{d}}{\mathrm{d} t} \frac{\partial L}{\partial \dot{\eta}_{i}}-\frac{\partial L}{\partial \eta_{i}}=Q_{i}, \quad i=1,2,3
$$

The generalized non-conservative friction forces $Q_{i}$ with respect to the generalized coordinates $\eta_{1}, \eta_{2}$, and $\eta_{3}$ are modeled with linear expressions:

$$
Q_{1}=-d_{1} \dot{\eta}_{1}+d_{2}\left(\dot{\eta}_{2}-\dot{\eta}_{1}\right), \quad Q_{2}=d_{2}\left(\dot{\eta}_{1}-\dot{\eta}_{2}\right)+d_{3}\left(\dot{\eta}_{3}-\dot{\eta}_{2}\right), \quad Q_{3}=d_{3}\left(\dot{\eta}_{2}-\dot{\eta}_{3}\right),
$$

whereby the parameters $d_{i}$ denote the damping coefficient at the respective joint $i$. The equations of motion (4.41) can be written in the matrix notation

$$
G \ddot{\boldsymbol{\eta}}+\boldsymbol{g}=\mathbf{0}
$$

with the matrix $G$ and the vector $\boldsymbol{g}$ depending on $\boldsymbol{\eta}=\left[\eta_{1}, \eta_{2}, \eta_{3}\right]^{\top}, \dot{\boldsymbol{\eta}}$, and $\ddot{y}$. The matrix $G$ and the elements of $\boldsymbol{g}=\left[g_{1}, g_{2}, g_{3}\right]^{\top}$ are given in Table 4.2.

As explained in Section 2.4.3, the experimental setup of the pendulum uses the cart acceleration $\ddot{y}$ as input $u$. Hence, the overall system model can be written as the input-output normal form $(2.18)-(2.19)$

$$
\begin{aligned}
\ddot{y} & =u \\
\ddot{\boldsymbol{\eta}} & =-G^{-1} \boldsymbol{b}=\boldsymbol{\beta}(\boldsymbol{\eta}, \dot{\boldsymbol{\eta}}, u)
\end{aligned}
$$

with relative degree $r=2, \boldsymbol{\beta}=\left[\beta_{1}, \beta_{2}, \beta_{3}\right]^{\top}$, and the system order $n=8$.

\subsubsection{Side-stepping problem}

The side-stepping maneuver within a finite time interval $t \in[0, T]$ requires to steer the triple inverted pendulum from the initial equilibrium

$$
y(0)=y_{0}^{*}, \quad \dot{y}(0)=0, \quad \boldsymbol{\eta}(0)=\dot{\boldsymbol{\eta}}(0)=\mathbf{0}
$$

to the terminal one

$$
y(T)=y_{T}^{*}, \quad \dot{y}(T)=0, \quad \boldsymbol{\eta}(T)=\dot{\boldsymbol{\eta}}(T)=\mathbf{0},
$$




$$
\begin{aligned}
G= & {\left[\begin{array}{ccc}
J_{1}+a_{1}^{2} m_{1}+l_{1}^{2}\left(m_{2}+m_{3}\right) & l_{1}\left(a_{2} m_{2}+l_{2} m_{3}\right) \cos \left(\eta_{1}-\eta_{2}\right) & a_{3} l_{1} m_{3} \cos \left(\eta_{1}-\eta_{3}\right) \\
l_{1}\left(a_{2} m_{2}+l_{2} m_{3}\right) \cos \left(\eta_{1}-\eta_{2}\right) & J_{2}+a_{2}^{2} m_{2}+l_{2}^{2} m_{3} & a_{3} l_{2} m_{3} \cos \left(\eta_{2}-\eta_{3}\right) \\
a_{3} l_{1} m_{3} \cos \left(\eta_{1}-\eta_{3}\right) & a_{3} l_{2} m_{3} \cos \left(\eta_{2}-\eta_{3}\right) & J_{3}+a_{3}^{2} m_{3}
\end{array}\right] } \\
g_{1}= & d_{1} \dot{\eta}_{1}+d_{2}\left(\dot{\eta}_{1}-\dot{\eta}_{2}\right)+a_{3} l_{1} m_{3} \sin \left(\eta_{1}-\eta_{3}\right) \dot{\eta}_{3}^{2}-\left[a_{1} m_{1}+l_{1}\left(m_{2}+m_{3}\right)\right]\left[g \sin \left(\eta_{1}\right)+\cos \left(\eta_{1}\right) \ddot{y}\right] \\
& +l_{1}\left(a_{2} m_{2}+l_{2} m_{3}\right) \sin \left(\eta_{1}-\eta_{2}\right) \dot{\eta}_{2}^{2} \\
g_{2}= & d_{2}\left(\dot{\eta}_{2}-\dot{\eta}_{1}\right)+d_{3}\left(\dot{\eta}_{2}-\dot{\eta}_{3}\right)-\left[a_{2} m_{2}+l_{2} m_{3}\right]\left[g \sin \left(\eta_{2}\right)+l_{1} \sin \left(\eta_{1}-\eta_{2}\right) \dot{\eta}_{1}^{2}+\cos \left(\eta_{2}\right) \ddot{y}\right] \\
& +a_{3} l_{2} m_{3} \sin \left(\eta_{2}-\eta_{3}\right) \dot{\eta}_{3}^{2} \\
g_{3}= & d_{3}\left(\dot{\eta}_{3}-\dot{\eta}_{2}\right)-a_{3} m_{3}\left[g \sin \left(\eta_{3}\right)+l_{1} \sin \left(\eta_{1}-\eta_{3}\right) \dot{\eta}_{1}^{2}+l_{2} \sin \left(\eta_{2}-\eta_{3}\right) \dot{\eta}_{2}^{2}+\cos \left(\eta_{3}\right) \ddot{y}\right]
\end{aligned}
$$

Table 4.2: Matrix $G$ and vector $\boldsymbol{g}=\left[g_{1}, g_{2}, g_{3}\right]^{\top}$ for the equations of motion (4.42) of the triple pendulum in Figure 4.6.

whereby both upward equilibria are unstable. The total displacement of the cart is $y_{T}^{*}-$ $y_{0}^{*}$. The feedforward control $\Sigma_{F F}$ in Figure 1.2 has to provide a nominal input trajectory $u^{*}(t)$ which realizes the side-stepping in open-loop. In order to achieve a fast and aggressive transition of the triple pendulum, the cart constraints (2.41) shall be considered directly within the feedforward control design, whereby sufficient control margin is required for the feedback control. Experiments with the triple pendulum have shown that the stabilization of the upward position leads to an oscillating input with maximum accelerations of up to $\Delta u=5 \mathrm{~m} / \mathrm{s}^{2}$ and maximal values of the velocity $\Delta \dot{y}=0.5 \mathrm{~m} / \mathrm{s}$ and cart displacement $\Delta y=0.05 \mathrm{~m}$ (Treuer, 2005). These values are taken into account by assuming the following constraints for the nominal output trajectory $y^{*}(t)$ :

$$
\begin{aligned}
& y^{*} \in\left[y_{0}^{-}, y_{0}^{+}\right]=[-0.65,0.65] \mathrm{m}, \\
& \dot{y}^{*} \in\left[y_{1}^{-}, y_{1}^{+}\right]=\left[\begin{array}{ll}
-2.0, & 2.0
\end{array}\right] \mathrm{m} / \mathrm{s}, \\
& \ddot{y}^{*} \in\left[y_{2}^{-}, y_{2}^{+}\right]=\left[\begin{array}{ll}
-15, & 15
\end{array}\right] \mathrm{m} / \mathrm{s}^{2} .
\end{aligned}
$$

\subsubsection{Feedforward control design}

The feedforward control (2.26) follows from inverting the input-output dynamics (2.18). In analogy to the swing-up of the double pendulum in Section 2.4, the feedforward control

$$
u^{*}(t)=\ddot{y}^{*}(t)
$$

is simply the second time derivative of the output trajectory $y^{*}(t)$. Thereby, $y^{*}(t)$ has to satisfy the output BCs in (4.45)-(4.46) to move the pendulum from the stationary position $y_{0}^{*}$ to the destination $y_{T}^{*}$. The trajectory $\boldsymbol{\eta}^{*}(t)$ of the angles can be obtained in a straightforward manner by solving the BVP (4.44) of the internal dynamics with the respective BCs in (4.45)-(4.46), which corresponds to the solution of the swing-up problem of the double pendulum. However, in this way the output constraints (4.47) are only satisfied by choosing the transition time $T$ long enough. 


\subsubsection{Boundary value problems for constrained output}

Following the approach derived in this chapter, the output constraints (4.47) are incorporated in the transition problem by formulating the BVPs (4.18)-(4.19). Hence, the internal dynamics (4.19) of the triple pendulum is extended by the new system (4.18) of order $r=2$ with the coordinates $\boldsymbol{\xi}_{2}=\left[\xi_{1}, \xi_{2}\right]^{\top}$ and the new input $v$ :

$$
\begin{aligned}
& \dot{\xi}_{1}=\psi_{2}\left(\xi_{2}, \psi_{2}^{ \pm}\left(\xi_{1}\right)\right), \quad \xi_{1}(0)=\psi_{1}^{-1}\left(y_{0}^{*}, \psi_{1}^{ \pm}\right), \quad \xi_{1}(T)=\psi_{1}^{-1}\left(y_{T}^{*}, \psi_{1}^{ \pm}\right), \\
& \dot{\xi}_{2}=\psi_{3}\left(v, \psi_{2}^{ \pm}\left(\boldsymbol{\xi}_{2}\right)\right), \quad \xi_{2}(0)=0, \quad \xi_{2}(T)=0, \\
& \ddot{\boldsymbol{\eta}}^{*}=\boldsymbol{\beta}\left(\boldsymbol{\eta}^{*}, \dot{\boldsymbol{\eta}}^{*}, \ddot{y}^{*}\right), \quad \boldsymbol{\eta}^{*}(0)=\dot{\boldsymbol{\eta}}^{*}(0)=0, \quad \boldsymbol{\eta}^{*}(T)=\dot{\boldsymbol{\eta}}^{*}(T)=0 .
\end{aligned}
$$

The output $y^{*}$ and its time derivatives $\dot{y}^{*}$ and $\ddot{y}^{*}=u^{*}$ are determined by the algebraic equations (4.3) and (4.14), i.e.

$$
\begin{aligned}
y^{*} & =\psi_{1}\left(\xi_{1}, \psi_{1}^{ \pm}\right), \\
\dot{y}^{*} & =\frac{\partial \psi_{1}}{\partial \xi_{1}} \psi_{2}\left(\xi_{2}, \psi_{2}^{ \pm}\left(\xi_{1}\right)\right), \\
\ddot{y}^{*} & =\frac{\partial^{2} \psi_{1}}{\partial \xi_{1}^{2}} \psi_{2}^{2}+\frac{\partial \psi_{1}}{\partial \xi_{1}} \frac{\partial \psi_{2}}{\partial \xi_{1}} \psi_{2}+\frac{\partial \psi_{1}}{\partial \xi_{1}} \frac{\partial \psi_{2}}{\partial \xi_{2}} \psi_{3}\left(v, \psi_{3}^{ \pm}\left(\boldsymbol{\xi}_{2}\right)\right) .
\end{aligned}
$$

Thereby, the relation $(4.52 \mathrm{c})$ for $\ddot{y}^{*}$ couples the coordinates $\xi_{1}$ and $\xi_{2}$ of the subsystem (4.49)(4.50) to the internal dynamics (4.51). The boundary values $\xi_{1,0}$ and $\xi_{1, T}$ in (4.49) depend on the initial and final cart position $y_{0}^{*}$ and $y_{T}^{*}$ according to (4.20). The BCs for $\xi_{2}$ are homogeneous due to the symmetric constraints (4.47b), see Section 4.2.2.

The saturation functions $\psi_{1}\left(\xi_{1}, \psi_{1}^{ \pm}\right)$and $\psi_{2}\left(\xi_{2}, \psi_{2}^{ \pm}\left(\xi_{1}\right)\right)$ are constructed with the asymptotic functions (4.12), whereas the ramp function (4.13) is used for $\psi_{3}\left(v, \psi_{3}^{ \pm}\left(\boldsymbol{\xi}_{2}\right)\right)$. The respective saturation limits are determined by (4.17), i.e.

$$
\psi_{1}^{ \pm}=y_{0}^{ \pm}, \quad \psi_{2}^{ \pm}\left(\xi_{1}\right)=y_{1}^{ \pm}\left[\frac{\partial \psi_{1}}{\partial \xi_{1}}\right]^{-1}, \quad \psi_{3}^{ \pm}\left(\boldsymbol{\xi}_{2}\right)=\frac{y_{2}^{ \pm}-\frac{\partial^{2} \psi_{1}}{\partial \xi_{1}^{2}} \psi_{2}^{2}-\frac{\partial \psi_{1}}{\partial \xi_{1}} \frac{\partial \psi_{2}}{\partial \xi_{1}} \psi_{2}}{\frac{\partial \psi_{1}}{\partial \xi_{1}} \frac{\partial \psi_{2}}{\partial \xi_{2}}}
$$

The solution of the BVPs (4.49)-(4.51) with $2 n=16$ BCs for $n=8$ first-order ODEs requires $n=8$ free parameters $\boldsymbol{p}=\left(p_{1}, \ldots, p_{8}\right)$, which are provided in the setup function $v=\Phi(t, \boldsymbol{p})$ to parameterize the new input $v$. As pointed out in Remark 2.6 for the swing-up of the double pendulum, a $\mathcal{C}^{1}$-continuous output trajectory $y^{*}(t)$ is sufficient for the experimental implementation, since the internal PI controller tracks the velocity profile $\dot{y}^{*}(t)$ instead of the acceleration $\ddot{y}^{*}(t)$. Hence, the polynomial (3.6a) is used to parameterize $\Phi(t, \boldsymbol{p})$ leading to a discontinuous feedforward control (4.48) at the transition bounds $t=0$ and $t=T$. 


\subsubsection{Numerical results}

The BVPs (4.49)-(4.51) with (4.52)-(4.53) and the free parameters $\boldsymbol{p}=\left(p_{1}, \ldots, p_{8}\right)$ in the setup function $v=\Phi(t, \boldsymbol{p})$ are solved with the MATLAB function bvp4c. A linear interpolation between the corresponding BCs on a uniform mesh with 30 grid points $t_{k} \in[0, T]$ serves as a reasonable guess for the trajectories $\xi_{1}\left(t_{k}\right), \xi_{2}\left(t_{k}\right)$, and $\boldsymbol{\eta}^{*}\left(t_{k}\right)$. The initial guess for the unknown parameters is $\boldsymbol{p}=\mathbf{0}$. The robustness and convergence of the numerical solution are enhanced by providing the analytical Jacobian matrix of the ODEs (4.49)-(4.51) to the bvp4c-function.

Figure 4.7 shows the computed cart trajectories (4.52) and the angles $\boldsymbol{\eta}^{*}(t)=\left[\eta_{1}^{*}(t), \eta_{2}^{*}(t), \eta_{3}^{*}(t)\right]^{\top}$ for a side-stepping over the distance $y_{T}^{*}-y_{0}^{*}=1 \mathrm{~m}$ (setpoints $y_{0}^{*}=-0.5 \mathrm{~m}$ and $y_{T}^{*}=0.5 \mathrm{~m}$ ) with the transition time $T=2.4 \mathrm{~s}$. For the calculation of the unconstrained case, the output constraints (4.47) are increased by the factor 10, in order to set $y_{0}^{ \pm}, y_{1}^{ \pm}$, and $y_{2}^{ \pm}$to sufficiently large values. The trajectories (- $)$ in Figure 4.7 clearly show that the actual constraints in (4.47a) and (4.47b) are violated by $\max _{t} y^{*}(t)=0.74 \mathrm{~m}$ and $\max _{t} \dot{y}^{*}(t)=3 \mathrm{~m} / \mathrm{s}$.

In the constrained case (-), the BVPs (4.49)-(4.51) are solved with respect to the constraints (4.47). The trajectories are significantly replanned compared to the unconstrained trajectories $(--)$. Especially the acceleration $\ddot{y}^{*}(t)$ shows a highly aggressive behavior. At the transition borders $t=0$ and $t=T$, the cart starts and stops with the maximal acceleration $y_{2}^{ \pm}=15 \mathrm{~m} / \mathrm{s}^{2}$ in order to perform this fast transition. ${ }^{6}$

It is also remarkable that the constraints $y_{0}^{ \pm}= \pm 0.65 \mathrm{~m}$ and $y_{1}^{ \pm}= \pm 15 \mathrm{~m} / \mathrm{s}$ are well exploited, although the saturation functions $\psi_{1}\left(\xi_{1}, \psi_{1}^{ \pm}\right)$and $\psi_{2}\left(\xi_{2}, \psi_{2}^{ \pm}\left(\xi_{1}\right)\right)$ touch the respective limits $\psi_{1}^{ \pm}$ and $\psi_{2}^{ \pm}\left(\xi_{1}\right)$ only asymptotically. The minimal distance of $y^{*}(t)$ and $\dot{y}^{*}(t)$ to the constraints $y_{0}^{ \pm}$ and $y_{1}^{ \pm}$is of order $10^{-4}$ and $10^{-9}$, respectively.

The good exploitation of the output constraints (4.47) is also confirmed in Figure 4.8, where the coordinates $\xi_{1}(t), \xi_{2}(t)$ are shown together with the saturation functions $\psi_{2}\left(\xi_{2}, \psi_{2}^{ \pm}\left(\xi_{1}\right)\right)$, $\psi_{3}\left(v, \psi_{3}^{ \pm}\left(\boldsymbol{\xi}_{2}\right)\right)$ and the partial derivatives $\partial^{i} \psi_{1} / \partial \xi_{1}^{i}, i=1,2$ and $\partial \psi_{2} / \partial \xi_{2}$. In the time intervals when $y^{*}(t)$ or $\dot{y}^{*}(t)$ stay at the constraints $y_{0}^{ \pm}$or $y_{1}^{ \pm}$, the respective saturation functions $\psi_{1}\left(\xi_{1}, \psi_{1}^{ \pm}\right)$or $\psi_{2}\left(\xi_{2}, \psi_{2}^{ \pm}\left(\xi_{1}\right)\right)$ approach one of their limits $\psi_{1}^{ \pm}$or $\psi_{2}^{ \pm}\left(\xi_{1}\right)$, such that the gradients $\partial^{i} \psi_{1} / \partial \xi_{1}^{i}, i=1,2$ and $\partial \psi_{2} / \partial \xi_{2}$ are almost zero.

The output constraints (4.52) are also illustrated by the time-discrete snapshots of the sidestepping maneuver in Figure 4.9. During the time interval $t \in[0.5 \mathrm{~s}, 0.8 \mathrm{~s}]$ at the beginning of the transition, the cart "waits" at the limit $y_{0}^{-}=-0.65 \mathrm{~m}$ until the three pendulum links fall in the direction of the side-stepping. In the following, the cart moves with maximum velocity $y_{1}^{+}=2 \mathrm{~m} / \mathrm{s}$ and finally "waits" again for the pendulum links at the maximum position $y_{0}^{+}=0.65 \mathrm{~m}$.

\footnotetext{
${ }^{6}$ If the new input $v=\Phi(t, \boldsymbol{p})$ in (4.50) is parameterized by the cosine series (3.6b) instead of the polynomial (3.6c), the steps in the acceleration $\ddot{y}^{*}(t)$ at $t=0$ and $t=T$ are smaller, but the side-stepping time $T$ must be increased to $T=2.7 \mathrm{~s}$ to find a solution of the BVPs (4.49)-(4.51). Alternatively, spline functions can be used to parameterize $v=\Phi(t, \boldsymbol{p})$, which allows to condense the "activity" of $\Phi(t, \boldsymbol{p})$ in a desired region, e.g. at the start and stop of the transition (Treuer, 2005; Graichen et al., 2005c).
} 

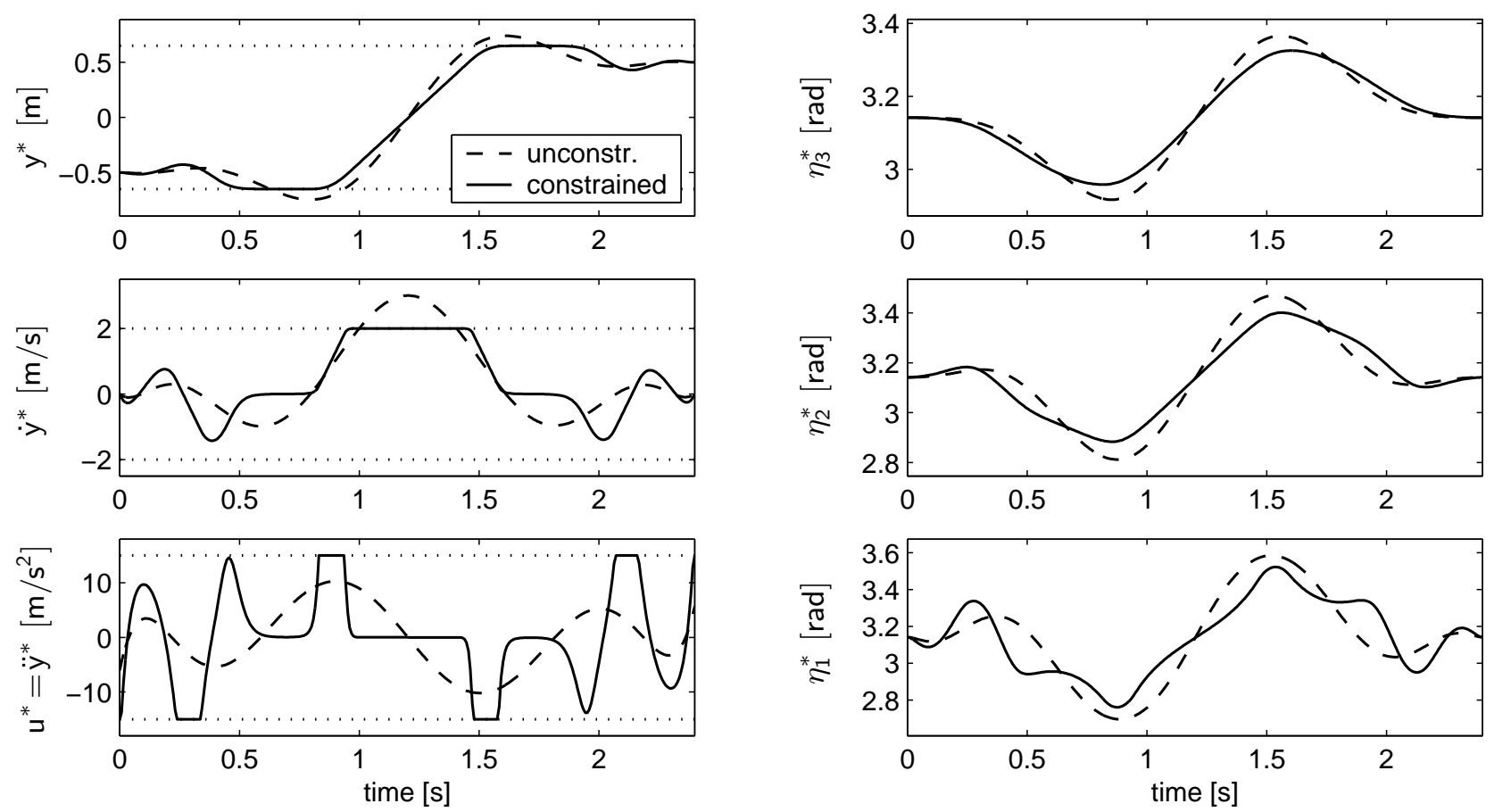

Figure 4.7: Nominal (un)constrained trajectories of the cart $y^{*}(t), \dot{y}^{*}(t), \ddot{y}^{*}(t)=u^{*}(t)$, and the angles $\eta_{1}^{*}(t), \eta_{2}^{*}(t), \eta_{3}^{*}(t)$ for the side-stepping of the triple inverted pendulum over the distance $y_{T}^{*}-y_{0}^{*}=1 \mathrm{~m}$ with the transition time $T=2.4 \mathrm{~s}$.
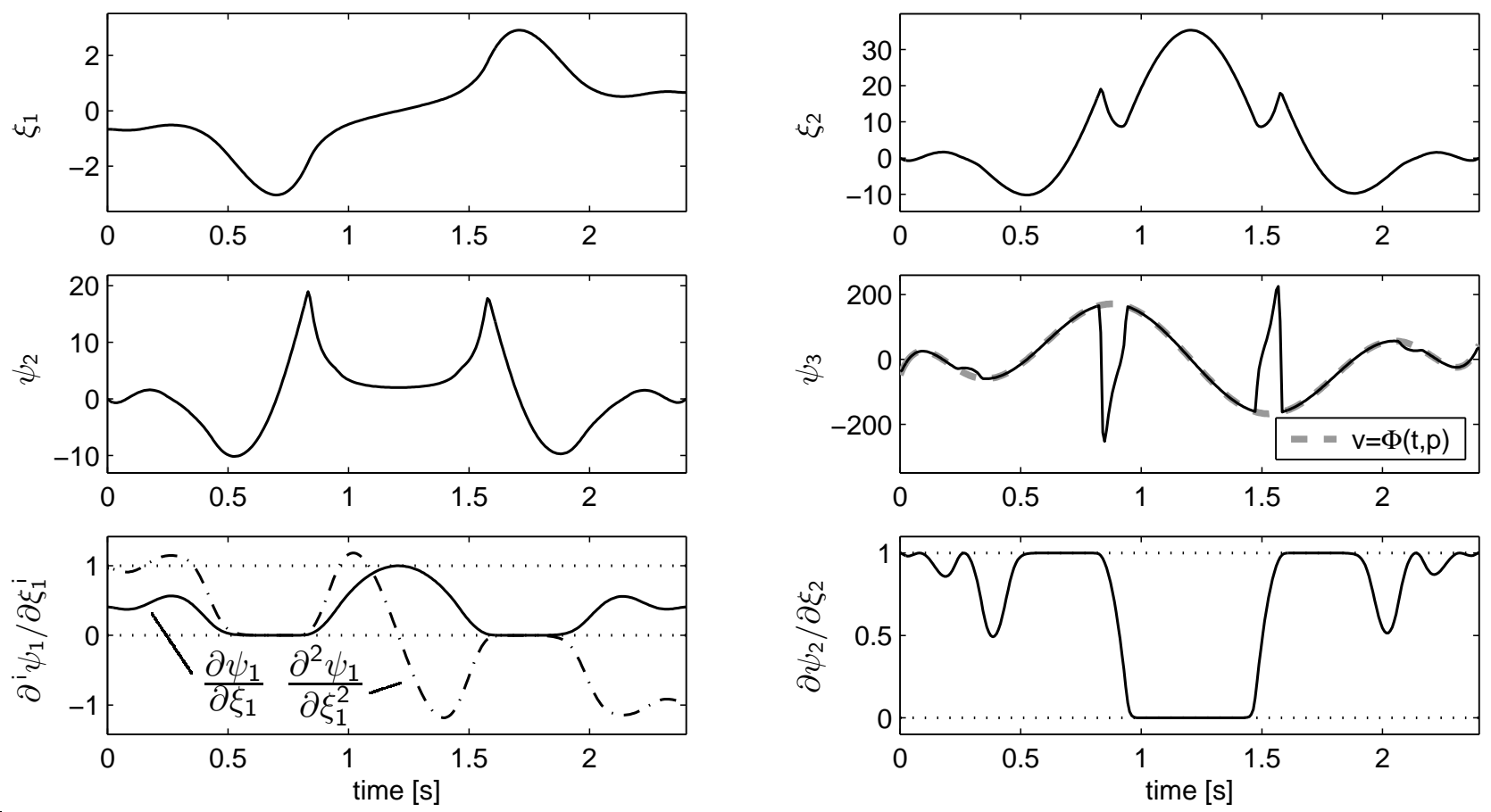

Figure 4.8: Trajectories of the coordinates $\xi_{1}(t), \xi_{2}(t)$ and saturation functions $\psi_{2}\left(\xi_{2}, \psi_{2}^{ \pm}\left(\xi_{1}\right)\right)$, $\psi_{3}\left(v, \psi_{3}^{ \pm}\left(\boldsymbol{\xi}_{2}\right)\right)$, as well as the partial derivatives $\partial^{i} \psi_{1} / \partial \xi_{1}^{i}, i=1,2$ and $\partial \psi_{2} / \partial \xi_{2}$ corresponding to the side-stepping of the triple inverted pendulum in Figure 4.7. 
Remark 4.4. The output trajectories in Figure 4.7 show that in the time intervals when $y^{*}(t)$ or $\dot{y}^{*}(t)$ approach the respective constraints $y_{0}^{ \pm}$or $y_{1}^{ \pm}$, the higher output derivatives up to $\ddot{y}^{*}(t)$ tend to zero. This can be easily explained for $y^{*} \rightarrow y_{0}^{ \pm}$, since in this case the first saturation function $y^{*}=\psi_{1}\left(\xi_{1}, \psi_{1}^{ \pm}\right)$approaches the limits $\psi_{1}^{ \pm}=y_{0}^{ \pm}$such that the vanishing gradients $\partial^{i} \psi_{1} / \partial \xi_{1}^{i} \rightarrow 0, i=1,2$ (see Figure 4.8) force the output derivatives (4.52b) and (4.52c) to zero. Correspondingly, if $\dot{y}^{*}=\frac{\partial \psi_{1}}{\partial \xi_{1}} \psi_{2}\left(\xi_{2}, \psi_{2}^{ \pm}\left(\xi_{1}\right)\right)$ reaches one of the constraints $y_{1}^{ \pm}$, the higher derivative $\ddot{y}^{*}$ tends to zero. This is due to the second saturation function $\psi_{2}\left(\xi_{2}, \psi_{2}^{ \pm}\left(\xi_{1}\right)\right)$, which approaches the respective limit $\psi_{2}^{ \pm}\left(\xi_{1}\right)=y_{1}^{ \pm}\left[\frac{\partial \psi_{1}}{\partial \xi_{1}}\right]^{-1}$, see (4.53). Hence, the partial derivatives of $\psi_{2}\left(\xi_{2}, \psi_{2}^{ \pm}\left(\xi_{1}\right)\right) \rightarrow \psi_{2}^{ \pm}\left(\xi_{1}\right)$ approach the following values:

$$
\frac{\partial \psi_{2}}{\partial \xi_{1}} \rightarrow-y_{1}^{ \pm}\left[\frac{\partial \psi_{1}}{\partial \xi_{1}}\right]^{-2} \frac{\partial^{2} \psi_{1}}{\partial \xi_{1}^{2}} \psi_{2}, \quad \frac{\partial \psi_{2}}{\partial \xi_{2}} \rightarrow 0 .
$$

Placing these expressions into Equation (4.52c) cancels out the first two terms, while the third term vanishes due to $\partial \psi_{2} / \partial \xi_{2} \rightarrow 0$. This shows that the second time derivative $\ddot{y}^{*} \rightarrow 0$ indeed tends to zero for $\dot{y}^{*} \rightarrow y_{1}^{ \pm}$.

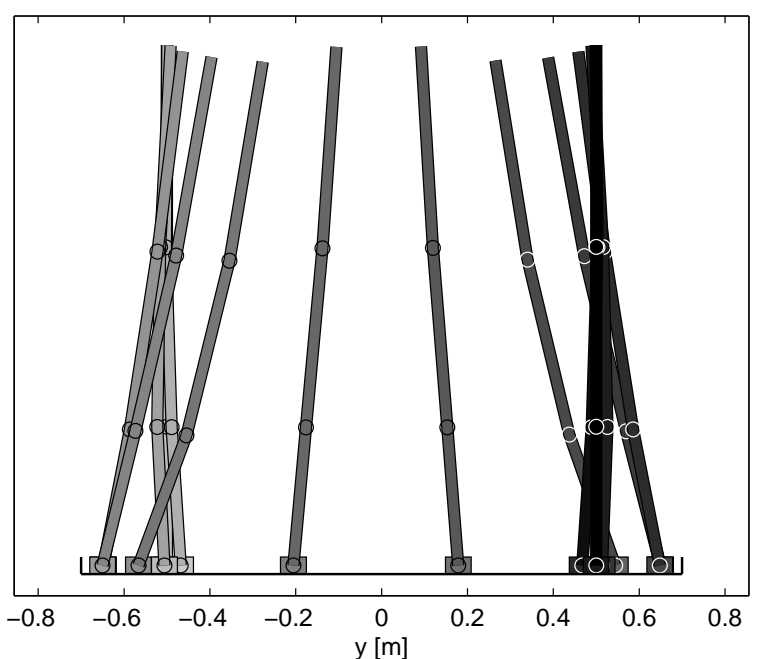

Figure 4.9: Snapshots of the nominal sidestepping for $T=2.4 \mathrm{~s}$ with increasing darkness of the snapshots as time increases during the transition.

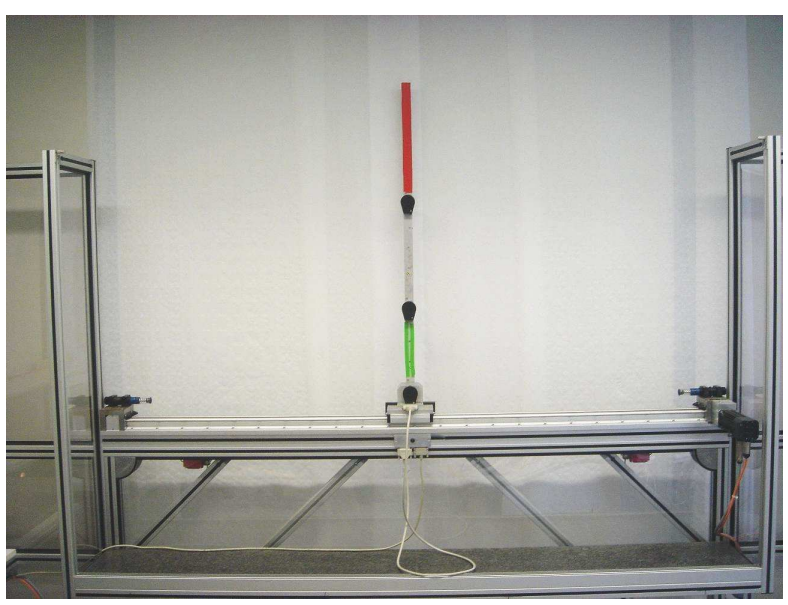

Figure 4.10: Experimental construction of the triple pendulum on a cart (Hasomed $\mathrm{GmbH}$ ) with model parameters in Table 2.1 and the cart constraints $(2.41)$

\subsubsection{Experimental validation}

The side-stepping of the triple inverted pendulum is experimentally validated with the same apparatus described in Section 2.4.3 for the swing-up of the double pendulum. Thereby, a third link is inserted between the two links of the double pendulum, cf. Figure 2.9 and 4.10. The implemented two-degree-of-freedom control in Figure 2.8 utilizes the state feedback control $\Sigma_{F B}$ supplied by the observer $\widehat{\Sigma}$ to stabilize the triple pendulum in the upward position. 
Due to the accuracy of the nonlinear feedforward control $\Sigma_{F F}$, the feedback part $\Sigma_{F B}$ is based on an LQ design with the pendulum model linearized in the upper unstable equilibrium. Similar to the feedback design for the swing-up of the double pendulum (see Section 2.4.3.3), the pendulum model in (4.43)-(4.44) is dynamically extended by the disturbance model $\dot{\tilde{y}}=y$ with the new state $\tilde{y}$, in order to compensate for a possible steady state error in the cart position $y$. Hence, the overall state vector comprises the states $\boldsymbol{x}=\left[y, \dot{y}, \boldsymbol{\eta}^{\top}, \dot{\boldsymbol{\eta}}^{\top}, \tilde{y}\right]^{\top} \in \mathbb{R}^{9}$ with $\boldsymbol{\eta}=\left[\eta_{1}, \eta_{2}, \eta_{3}\right]^{\top}$. A Luenberger observer $\widehat{\Sigma}$ (O'Reilly, 1983) based on the nonlinear model (2.44)(2.45) provides the state estimation $\hat{\boldsymbol{x}}$ for the state feedback control $\widehat{\Sigma}_{F B}$. The error dynamics of the observer is designed by eigenvalue assignment with the pendulum model linearized along the nominal trajectories $\hat{\boldsymbol{x}}(t)$ (Treuer, 2005). The feedforward control $u^{*}(t)$ and the nominal trajectories $\boldsymbol{x}^{*}(t)=\left[y^{*}(t), \dot{y}^{*}(t), \boldsymbol{\eta}^{* \top}(t), \dot{\boldsymbol{\eta}}^{* \top}(t), \tilde{y}^{*}(t)\right]^{\top}$ with $\tilde{y}^{*}(t)=\int_{0}^{t} y^{*}(\tau) \mathrm{d} \tau$ are calculated offline and stored in look-up tables.

Figure 4.11 shows the experimental and nominal trajectories of the angles $\eta_{i}(t), i=1,2,3$, the cart position $y(t)$ and velocity $\dot{y}(t)$ as well as the input $u(t)=\ddot{y}(t)$ for the side-stepping of $y_{T}^{*}-y_{0}^{*}=1 \mathrm{~m}$ in the transition time $T=2.4 \mathrm{~s}$. The profiles reveal the good tracking behavior. The deviations of the input $u(t)$ in closed-loop compared to the feedforward control $u^{*}(t)$ are mainly due to model inaccuracies and the response time of the underlying PI controller for the
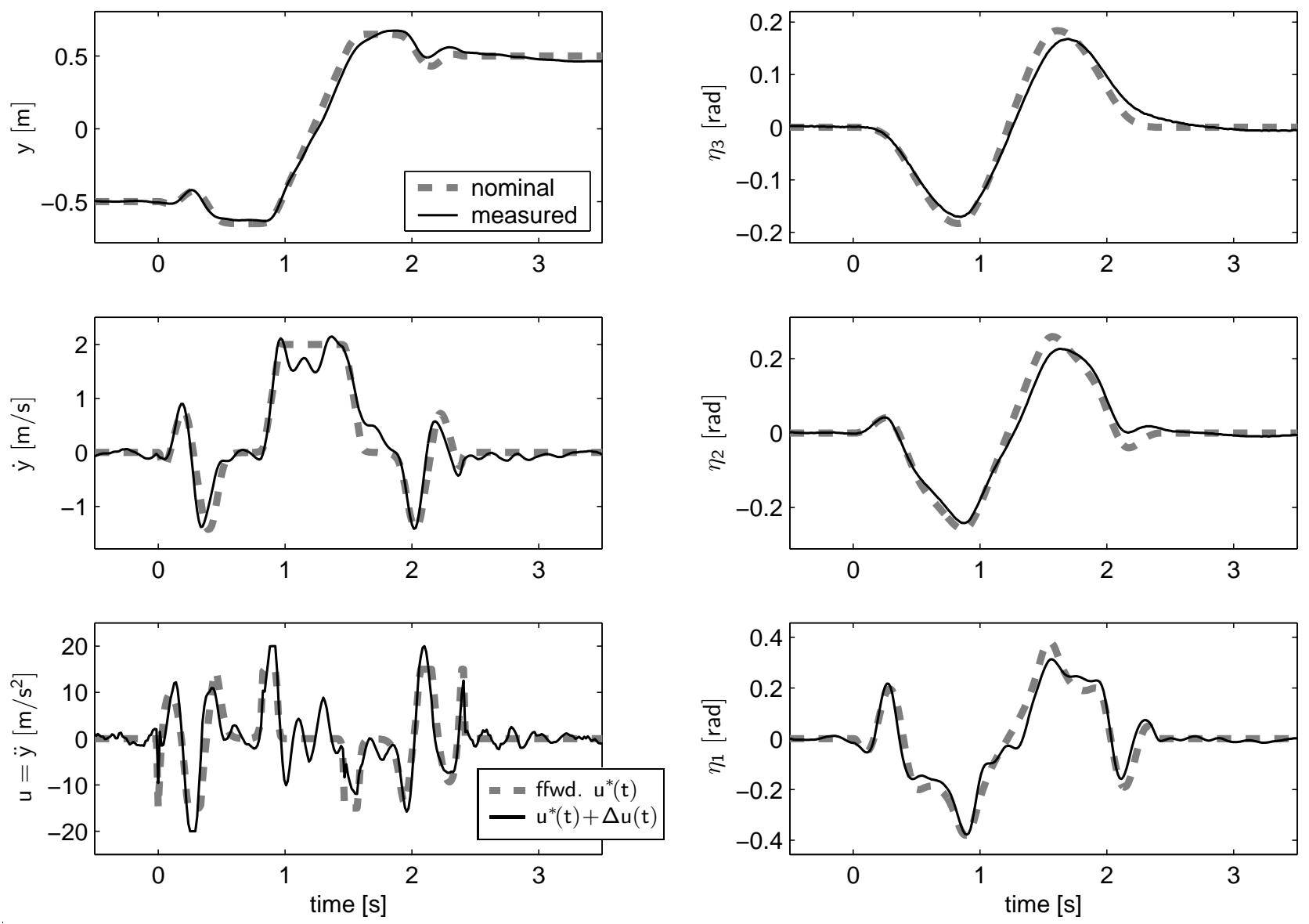

Figure 4.11: Experimental and nominal trajectories for the side-stepping of the triple inverted pendulum by $1 \mathrm{~m}$ in the transition time $T=2.4 \mathrm{~s}$. 
cart acceleration $u=\ddot{y}$ (see Remark 2.6), which has to deal with the aggressive feedforward control $u^{*}(t)$ to perform the transition in $T=2.4 \mathrm{~s}$. Nevertheless, the overall control $u(t)=$ $u^{*}(t)+\Delta u(t)$ clearly shows that the side-stepping is primarily performed by the feedforward control $u^{*}(t)$, whereas the feedback part $\Delta u(t)$ stabilizes the pendulum along the nominal trajectories. Thereby, the stabilization of the triple pendulum in the unstable upward position leads to a permanent input oscillation of approximately $\pm 3 \mathrm{~m} / \mathrm{s}^{2}$.

The tracking performance can be further enhanced by increasing the transition time $T$ e.g. to $T=3 \mathrm{~s}$, which leads to a less aggressive side-stepping maneuver (Treuer, 2005; Graichen et al., 2005c). Moreover, an alternative to the implemented LQ feedback control with the constant gain vector $\boldsymbol{k} \in \mathbb{R}^{9}$ is the reverse-time solution of the Riccati ODE (2.56) in Section 2.4.3.3, in order to compute time-varying gains $\boldsymbol{k}(t) \in \mathbb{R}^{9}$ for the feedback correction $\Delta u=\boldsymbol{k}^{\top}(t)\left(\boldsymbol{x}^{*}-\boldsymbol{x}\right)$ in $(2.54)$.

\subsection{Conclusions}

The feedforward control design for nonlinear SISO systems has been extended to account for constraints on the output trajectory and its time derivatives. The basic idea is to construct a new system representation for the constrained output trajectory by means of saturation functions and successive differentiation of the output. The output constraints are incorporated by appropriately determining the limits of the single saturation functions. The corresponding BVP of the newly constructed system replaces the previous output BVP and is solved together with the internal dynamics BVP. In this way, the output constraints are systematically incorporated in the formulation of the BVPs for the transition problem. Moreover, the input constraints addressed in the previous chapter can be projected to constraints for the highest output derivative by means of the input-output dynamics.

This concept is illustrated for the side-stepping of the triple inverted pendulum with constraints on the cart position, velocity, and acceleration. Thereby, a remarkable feature is that the BVP solution is obtained in a straightforward manner for a simple initial guess with linear interpolations for $\xi_{1}(t), \xi_{2}(t)$ and $\boldsymbol{\eta}(t)$ between the respective BCs, and the guess $\boldsymbol{p}=\mathbf{0}$ for the parameters. Although the model of the triple inverted pendulum is highly nonlinear and unstable, the systematic incorporation of the output constraints within the feedforward control design maintains the good numerical conditioning of the BVPs in combination with algebraic solution techniques like finite difference or collocation methods (e.g. bvp4c) that are not based on a numerical integration of the ODEs. 


\section{Chapter 5}

\section{Feedforward control design for nonlinear MIMO systems}

The feedforward control design under input and output constraints as presented in the previous three chapters can be extended to the multiple-input multiple-output (MIMO) case in a straightforward manner Graichen and Zeitz (2006a, 2007). In a first step, the finite-time transition problem between stationary setpoints is defined for nonlinear MIMO systems, before the inversion-based feedforward control design and the incorporation of constraints on the single input and output channels are addressed in the following sections. The feedforward control design is illustrated for a flight maneuver of a 3DOF helicopter with constraints on the two inputs and the pitch angle (Kiefer et al., 2006). By appropriately choosing the outputs, the pitch angle constraints are interpreted as output constraints in order to apply the feedforward control design to the 3DOF helicopter.

\subsection{Finite-time transition between stationary setpoints}

Considered are nonlinear MIMO systems

$$
\begin{aligned}
& \Sigma: \quad \dot{\boldsymbol{x}}=\boldsymbol{f}(\boldsymbol{x}, \boldsymbol{u}), \quad \boldsymbol{x}(0)=\boldsymbol{x}_{0} \\
& y_{k}=h_{k}(\boldsymbol{x}), \quad k=1, \ldots, m
\end{aligned}
$$

with time $t \in \mathbb{R}$, state $\boldsymbol{x} \in \mathbb{R}^{n}$, input vector $\boldsymbol{u} \in \mathbb{R}^{m}$, and $m$ outputs $y_{k} \in \mathbb{R}, k=1, \ldots, m$. The vector field $\boldsymbol{f}: \mathbb{R}^{n} \times \mathbb{R}^{m} \rightarrow \mathbb{R}^{n}$ and the functions $h_{k}: \mathbb{R}^{n} \rightarrow \mathbb{R}$ are assumed to be sufficiently smooth. ${ }^{1}$

A widespread control problem concerns the transition between two stationary setpoints $\left(\boldsymbol{u}_{0}^{*}, \boldsymbol{x}_{0}^{*}\right.$, $\left.y_{1,0}^{*}, \ldots, y_{m, 0}^{*}\right)$ and $\left(\boldsymbol{u}_{T}^{*}, \boldsymbol{x}_{T}^{*}, y_{1, T}^{*}, \ldots, y_{m, T}^{*}\right)$ of system $(5.1)$ within a finite time interval $t \in[0, T]$.

\footnotetext{
${ }^{1}$ In order to maintain consistency with the notation in the previous chapters, the index " $k$ " is used to signify the inputs $u_{k}$ and outputs $y_{k}, k=1, \ldots, m$.
} 
It is assumed that the stationary solutions

$$
\begin{array}{rrr}
\left(\boldsymbol{x}_{0}^{*}, \boldsymbol{u}_{0}^{*}\right): & \boldsymbol{f}\left(\boldsymbol{x}_{0}^{*}, \boldsymbol{u}_{0}^{*}\right)=\mathbf{0}, & y_{k, 0}^{*}=h_{k}\left(\boldsymbol{x}_{0}^{*}\right) \\
\left(\boldsymbol{x}_{T}^{*}, \boldsymbol{u}_{T}^{*}\right): & \boldsymbol{f}\left(\boldsymbol{x}_{T}^{*}, \boldsymbol{u}_{T}^{*}\right)=\mathbf{0}, & y_{k, T}^{*}=h_{k}\left(\boldsymbol{x}_{T}^{*}\right), \quad k=1, \ldots, m
\end{array}
$$

are uniquely determined. The transition between the setpoints $(5.2)$ in the time $t \in[0, T]$ means that the system (5.1) has to satisfy the boundary conditions (BCs)

$$
\boldsymbol{x}(0)=\boldsymbol{x}_{0}^{*}, \quad \boldsymbol{x}(T)=\boldsymbol{x}_{T}^{*},
$$

whereby the initial state $\boldsymbol{x}_{0}=\boldsymbol{x}_{0}^{*}$ has to be consistent with the stationary setpoint $\boldsymbol{x}_{0}^{*}$. From a mathematical point of view, the $n$ first-order differential equations in (5.1) and the $2 n$ BCs (5.3) form a two-point boundary value problem (BVP) for the states $\boldsymbol{x}=\left[x_{1}, \ldots, x_{n}\right]^{\top}$ in dependence of the input trajectories $u_{k}(t), k=1, \ldots, m$. Thereby, some controllability properties are required for the system (5.1) to accomplish the finite-time transition, which directly shows the importance of reasonably choosing the transition time $T$ with respect to the system dynamics (5.1) and possibly given constraints.

\subsubsection{Input-output normal form}

The inversion-based feedforward control design is based on the input-output representation of the considered system (Devasia et al., 1996; Chen and Paden, 1996; Graichen et al., 2005a). The derivation of the input-output coordinates of the MIMO system (5.1) requires the definition of the vector relative degree $\left\{r_{1}, \ldots, r_{m}\right\}$ :

Definition 5.1. The nonlinear MIMO system (5.1) has the vector relative degree $\left\{r_{1}, \ldots, r_{m}\right\}$ at point $\boldsymbol{x}^{0}$ if the following two conditions are fulfilled (Isidori, 1995; Nijmeijer and van der Schaft, 1990)

$$
\begin{aligned}
\frac{\partial}{\partial u_{j}} L_{\boldsymbol{f}}^{i} h_{k}(\boldsymbol{x})=0 & \forall j, k \in\{1, \ldots, m\}, \quad i=1, \ldots, r_{k}-1 \\
\frac{\partial}{\partial u_{j}} L_{\boldsymbol{f}}^{r_{k}} h_{k}(\boldsymbol{x}) \neq 0 & \text { for at least one } j \in\{1, \ldots, m\}
\end{aligned}
$$

for all admissible $\boldsymbol{u}=\left[u_{1}, \ldots, u_{m}\right]^{\top}$ and all $\boldsymbol{x}$ in a neighborhood of $\boldsymbol{x}^{0}$,

(ii) the $(m \times m)$ decoupling matrix

$$
A(\boldsymbol{x})=\frac{\partial}{\partial \boldsymbol{u}}\left[\begin{array}{c}
L_{\boldsymbol{f}}^{r_{1}} h_{1}(\boldsymbol{x}) \\
\vdots \\
L_{\boldsymbol{f}}^{r_{m}} h_{m}(\boldsymbol{x})
\end{array}\right]=\left[\begin{array}{ccc}
\frac{\partial}{\partial u_{1}} L_{\boldsymbol{f}}^{r_{1}} h_{1}(\boldsymbol{x}) & \ldots & \frac{\partial}{\partial u_{m}} L_{\boldsymbol{f}}^{r_{1}} h_{1}(\boldsymbol{x}) \\
\vdots & & \vdots \\
\frac{\partial}{\partial u_{1}} L_{\boldsymbol{f}}^{r_{m}} h_{m}(\boldsymbol{x}) & \ldots & \frac{\partial}{\partial u_{m}} L_{\boldsymbol{f}}^{r_{m}} h_{m}(\boldsymbol{x})
\end{array}\right]
$$

is nonsingular at $\boldsymbol{x}=\boldsymbol{x}^{0}$.

The operator $L_{\boldsymbol{f}}$ represents the Lie derivative along the vector field $\boldsymbol{f}$. 
Literally, the element $r_{k}$ of the vector relative degree $\left\{r_{1}, \ldots, r_{m}\right\}$ denotes the number of times the output $y_{k}$ has to be differentiated until at least one component $u_{k}, k=1, \ldots, m$ of the input vector $\boldsymbol{u}$ appears explicitly. ${ }^{2}$ In the following, it is assumed that the relative degree $\left\{r_{1}, \ldots, r_{m}\right\}$ is well-defined at least locally in the neighborhood of $\boldsymbol{x}^{0}$.

Remark 5.1. The rank condition for matrix (5.4c) is required for a static input-output linearization and decoupling of the system (5.1). If the matrix (5.4c) has a rank deficiency, the dynamic extension algorithm (Isidori, 1995) can be used to transform the system into a statically linearizable one.

The input-output coordinates of the system (5.1) are determined via the diffeomorphism (Isidori, 1995; Nijmeijer and van der Schaft, 1990)

$$
\left[\boldsymbol{y}_{1}^{\top}, \ldots, \boldsymbol{y}_{m}^{\top}, \boldsymbol{\eta}^{\top}\right]^{\top}=\phi(\boldsymbol{x})
$$

with the output derivatives

$$
\begin{aligned}
\boldsymbol{y}_{k} & =\left[y_{k}, \dot{y}_{k}, \ldots, y_{k}^{\left(r_{k}-1\right)}\right]^{\top} \in \mathbb{R}^{r_{k}} \\
& =\left[h_{k}(\boldsymbol{x}), L_{f} h_{k}(\boldsymbol{x}), \ldots, L_{f}^{r_{k}-1} h_{k}(\boldsymbol{x})\right]^{\top}, \quad k=1, \ldots, m
\end{aligned}
$$

and the supplementary state vector

$$
\boldsymbol{\eta}=\boldsymbol{\phi}_{\eta}(\boldsymbol{x}) \in \mathbb{R}^{n-r}, \quad r=\sum_{k=1}^{m} r_{k},
$$

to complete the diffeomorphism $\phi(x) \in \mathbb{R}^{n}$. With the coordinates (5.5), the MIMO system (5.1) can be transformed to the nonlinear input-output normal form ${ }^{3}$

$$
\begin{aligned}
\Sigma_{I O}: \quad y_{k}^{\left(r_{k}\right)} & =\alpha_{k}\left(\boldsymbol{y}_{1}, \ldots, \boldsymbol{y}_{m}, \boldsymbol{\eta}, \boldsymbol{u}\right), \quad k=1, \ldots, m \\
\dot{\boldsymbol{\eta}} & =\boldsymbol{\beta}\left(\boldsymbol{y}_{1}, \ldots, \boldsymbol{y}_{m}, \boldsymbol{\eta}, \boldsymbol{u}\right),
\end{aligned}
$$

with $\alpha_{k}(\cdot)=L_{f}^{r_{k}} h_{k} \circ \phi^{-1}$ and $\beta_{i}(\cdot)=L_{f} \phi_{\eta, i} \circ \phi^{-1}, i=1, \ldots, n-r$. The $m$ chains of $r_{k}$ integrators with input $\boldsymbol{u}$ appearing in the last equations (5.6) represent the input-output dynamics. The internal dynamics is defined by the ODE (5.7) for the state $\boldsymbol{\eta} \in \mathbb{R}^{n-r}$.

The BCs (5.3) of the considered transition problem can be transformed via the diffeomorphism (5.5) into the coordinates of the input-output normal form (5.6)-(5.7)

$$
\begin{aligned}
& y_{k}(0)=y_{k, 0}^{*}=h_{k}\left(\boldsymbol{x}_{0}^{*}\right), \quad y_{k}(T)=y_{k, T}^{*}=h_{k}\left(\boldsymbol{x}_{T}^{*}\right),\left.\quad y_{k}^{(i)}\right|_{t=0, T}=0, \\
& k=1, \ldots, m, \quad i=1, \ldots, \rho_{k} \in\left\{r_{k}-1, r_{k}\right\} \\
& \boldsymbol{\eta}(0)=\boldsymbol{\eta}_{0}^{*}=\boldsymbol{\phi}_{\eta}\left(\boldsymbol{x}_{0}^{*}\right), \quad \boldsymbol{\eta}(T)=\boldsymbol{\eta}_{T}^{*}=\boldsymbol{\phi}_{\eta}\left(\boldsymbol{x}_{T}^{*}\right) .
\end{aligned}
$$

\footnotetext{
${ }^{2}$ The original definition of the vector relative degree $\left\{r_{1}, \ldots, r_{m}\right\}$ in (Isidori, 1995; Nijmeijer and van der Schaft, 1990) is based on the input-affine representation of a nonlinear MIMO system, which is adapted here to the general nonlinear system (5.1).

${ }^{3}$ To simplify matters, the derivative vectors $\boldsymbol{y}_{k}=\left[y_{k}, \dot{y}_{k}, \ldots, y_{k}^{\left(r_{k}-1\right)}\right]^{\top}$ defined in (5.5b) are used in the following to denote the output coordinates.
} 
Thereby, the BVP (5.1)-(5.3) is split into into $m+1$ coupled BVPs (5.6)-(5.9) for $y_{k}, k=$ $1, \ldots, m$ and $\boldsymbol{\eta}$ in dependence of the inputs $u_{k}(t) \in[0, T]$. The symbol $\rho_{k}$ in (5.8) denotes the number of derivatives $y_{k}^{(i)}$ with homogeneous BCs. Thereby, the value $\rho_{k}=r_{k}-1$ corresponds to the number of output coordinates defined in (5.5). However, if all inputs $u_{k}(t)$ have to be continuous at the bounds $t=0$ and $t=T$, the inversion of (5.6) with respect to $\boldsymbol{u}$ reveals that the highest time derivatives $y_{k}^{\left(r_{k}\right)}, k=1, \ldots, m$ of the outputs have to satisfy the additional BCs $y_{k}^{\left(r_{k}\right)}(0)=y_{k}^{\left(r_{k}\right)}(T)=0$, which is denoted by $\rho_{k}=r_{k}$ in $(5.8)$.

Example 5.1. The following linear controllable system of third-order with two inputs and two outputs (i.e. $m=2$ ) is used to illustrate the feedforward control design in the MIMO case:

$$
\dot{\boldsymbol{x}}=\left[\begin{array}{rrr}
-2 & 0 & 1 \\
0 & -3 & 1 \\
-2 & 0 & -2
\end{array}\right] \boldsymbol{x}+\left[\begin{array}{rr}
1 & -1 \\
0 & 1 \\
1 & 2
\end{array}\right] \boldsymbol{u}, \quad \boldsymbol{y}=\left[\begin{array}{lll}
1 & 0 & 0 \\
0 & 1 & 0
\end{array}\right] \boldsymbol{x}
$$

The system has the vector relative degree $\{1,1\}$ and is already given in input-output normal form (5.6)-(5.7) with the third coordinate $\eta=x_{3}$ :

$$
\begin{aligned}
& \dot{y}_{1}=-2 y_{1}+\eta+u_{1}-u_{2}=\alpha_{1}\left(y_{1}, \eta, u_{1}, u_{2}\right) \\
& \dot{y}_{2}=-3 y_{2}+\eta+u_{2} \quad=\alpha_{2}\left(y_{2}, \eta, u_{2}\right) \\
& \dot{\eta}=-2 y_{1}-2 \eta+u_{1}+2 u_{2}=\beta\left(y_{1}, \eta, u_{1}, u_{2}\right) \text {. }
\end{aligned}
$$

The ODEs (5.11a)-(5.11b) and (5.11c) form the input-output dynamics and the internal dynamics. In order to steer the system (5.10) (or (5.11), respectively) between two stationary outputs $\left(y_{1,0}^{*}, y_{2,0}^{*}\right) \rightarrow\left(y_{1, T}^{*}, y_{2, T}^{*}\right)$ in the finite time interval $t \in[0, T]$ requires the following $B C s$ to be satisfied:

$$
\begin{aligned}
& y_{1}^{*}(0)=y_{1,0}^{*}, \quad y_{1}^{*}(T)=y_{1, T}^{*},\left.\quad \dot{y}_{1}^{*}\right|_{t=0, T}=0, \\
& y_{2}^{*}(0)=y_{2,0}^{*}, \quad y_{2}^{*}(T)=y_{2, T}^{*},\left.\quad \dot{y}_{2}^{*}\right|_{t=0, T}=0 \text {, } \\
& \eta^{*}(0)=\eta_{0}^{*}=\frac{3}{2} y_{2,0}^{*}, \quad \eta^{*}(T)=\eta_{T}^{*}=\frac{3}{2} y_{2, T}^{*} .
\end{aligned}
$$

The stationary boundary values $\eta_{0}^{*}$ and $\eta_{T}^{*}$ as well as the corresponding inputs $u_{1, i}^{*}=2 y_{1, i}^{*}$ and $u_{2, i}^{*}=\frac{3}{2} y_{2, i}^{*}, i=0, T$ follow from the stationary solution of the ODEs (5.11).

\subsubsection{Inversion-based feedforward control design}

The inversion-based feedforward control design (Devasia et al., 1996; Chen and Paden, 1996; Graichen et al., 2005a) is based on the inverse of the input-output dynamics (5.6)

$$
\boldsymbol{u}^{*}=\boldsymbol{\alpha}^{-1}\left(\boldsymbol{y}_{1}^{*}, y_{1}^{*\left(r_{1}\right)}, \ldots, \boldsymbol{y}_{m}^{*}, y_{m}^{*\left(r_{m}\right)}, \boldsymbol{\eta}^{*}\right)
$$

with $\boldsymbol{\alpha}=\left[\alpha_{1}, \ldots, \alpha_{m}\right]^{\top}$, which allows the explicit determination of the feedforward control $\boldsymbol{u}^{*}(t)$ with respect to the output trajectories $y_{k}^{*}(t), k=1, \ldots, m$ and the trajectory $\boldsymbol{\eta}^{*}(t)$ of the 
internal dynamics. ${ }^{4}$ The output trajectories $y_{k}^{*}(t) \in \mathcal{C}^{\rho_{k}}, k=1, \ldots, m$ have to satisfy the BCs (5.8), whereby $\rho_{k}=r_{k}$ holds if continuity of the feedforward control (5.13) is desired.

In order to determine the trajectory $\boldsymbol{\eta}^{*}(t)$ of the internal dynamics, the BVP (5.7), (5.9) can be rewritten by substituting the feedforward control (5.13) into (5.7), i.e.

$$
\dot{\boldsymbol{\eta}}^{*}=\overline{\boldsymbol{\beta}}\left(\boldsymbol{\eta}^{*}, \boldsymbol{y}_{1}^{*}, y_{1}^{*\left(r_{1}\right)}, \ldots, \boldsymbol{y}_{m}^{*}, y_{m}^{\left(r_{m}\right)}\right), \quad \boldsymbol{\eta}^{*}(0)=\boldsymbol{\eta}_{0}^{*}, \quad \boldsymbol{\eta}^{*}(T)=\boldsymbol{\eta}_{T}^{*}
$$

The outputs $y_{k}^{*}, k=1, \ldots, m$ and their $r_{k}$ time derivatives serve as inputs to (5.14). The corresponding zero dynamics is derived by zeroing the outputs and their time derivatives, i.e. $y_{k}^{*(i)}=0, i=0,1, \ldots, r_{k}, k=1, \ldots, m$ in (5.14). If the zero dynamics is stable, the system (5.1) is minimum-phase; it is nonminimum-phase, if the zero dynamics is unstable.

The BVP (5.14) of the internal dynamics is overdetermined by $2(n-r)$ BCs for $(n-r)$ ODEs. Following the approach described in Section 2.2.1 for the SISO case, the outputs $y_{k}^{*}$ are parameterized by $m$ setup functions

$$
y_{k}^{*}=\Upsilon_{k}\left(t, \boldsymbol{p}_{k}\right), \quad k=1, \ldots, m,
$$

which contain $q_{k}$ free parameters comprised in the sets

$$
\boldsymbol{p}_{k}=\left\{\begin{array}{ll}
\left(p_{k, 1}, \ldots, p_{k, q_{k}}\right) & \text { if } q_{k} \geq 1 \\
\emptyset & \text { else }
\end{array} \quad \text { with } \quad \sum_{k=1}^{m} q_{k}=n-r .\right.
$$

The overall number of $n-r$ free parameters corresponds to the order of the internal dynamics. Moreover, the functions $\Upsilon_{k}\left(t, \boldsymbol{p}_{k}\right)$ have to satisfy the respective BCs (5.8), i.e.

$$
\begin{aligned}
& \Upsilon_{k}\left(0, \boldsymbol{p}_{k}\right)=y_{k, 0}^{*}, \quad \Upsilon_{k}\left(T, \boldsymbol{p}_{k}\right)=y_{k, T}^{*},\left.\quad \Upsilon_{k}^{(i)}\right|_{t=0, T}=0, \\
& k=1, \ldots, m, \quad i=1, \ldots, \rho_{k} \in\left\{r_{k}-1, r_{k}\right\}
\end{aligned}
$$

with $\rho_{k}=r_{k}-1$ or $\rho_{k}=r_{k}$ depending on the desired continuity property of the input (5.13). In analogy to the SISO case, the functions $\Upsilon_{k}\left(t, \boldsymbol{p}_{k}\right)$ can be constructed e.g. with the polynomial or cosine series in (2.31), also see Appendix A.

A difference to the SISO case with only one output $y$ is that the $m$ numbers $q_{k}$ determine how the free parameters $\boldsymbol{p}_{k}$ are distributed over the setup functions $\Upsilon_{k}\left(t, \boldsymbol{p}_{k}\right)$. The choice of $q_{k}$ is a degree of freedom which has to be chosen with respect to the structure of the internal dynamics (5.14). For instance, if a certain output $y_{j}$ does not appear in the internal dynamics (5.14), then clearly no parameters can be provided in $y_{j}^{*}=\Upsilon_{j}(t)$, i.e. $q_{j}=0$.

Substituting the outputs (5.15) and their time derivatives into the internal dynamics (5.14) results in a BVP with free parameters, which can be solved e.g. with the standard MATLAB solver bvp4c (Section 2.3.2). Its solution comprises the trajectory $\boldsymbol{\eta}^{*}(t), t \in[0, T]$ and the parameter sets $\boldsymbol{p}_{k}$. The shape of the output trajectories $y_{k}^{*}(t)=\Upsilon_{k}\left(t, \boldsymbol{p}_{k}\right)$ is thus determined by the values of $\boldsymbol{p}_{k}$ in $\Upsilon_{k}\left(t, \boldsymbol{p}_{k}\right)$. Finally, the feedforward control $\boldsymbol{u}^{*}(t), \in[0, T]$ follows from (5.13) with the trajectories $y_{k}^{*}(t), k=1, \ldots, m$ and $\boldsymbol{\eta}^{*}(t)$.

\footnotetext{
${ }^{4}$ The inverse vector function $\boldsymbol{\alpha}^{-1}$ exists at least locally due to the rank condition (5.4c).
} 
Remark 5.2. In extension to Remark 5.1 and 2.2, another possibility to ensure that the decoupling matrix (5.4c) has full rank is to define different outputs $\tilde{y}_{k}=\widetilde{h}_{k}(\boldsymbol{x}), k=1, \ldots, m$ for the system (5.1). Since the shape of the output trajectories $y_{k}^{*}(t), k=1, \ldots, m$ is not predefined but determined by the setup (5.15) in order to solve the BVP (5.14), the definition of different outputs $\tilde{y}_{k}$ may be acceptable in order to achieve a well-defined relative degree.

Example 5.2 (Example 5.1 continued). The feedforward control (5.13) for the linear MIMO system (5.10) is obtained by inverting the input-output dynamics (5.11a)-(5.11b) with respect to $\boldsymbol{u}=\left[u_{1}, u_{2}\right]^{\top}$, i.e.

$$
\boldsymbol{u}^{*}=\left[\begin{array}{c}
2 y_{1}^{*}+\dot{y}_{1}^{*}+3 y_{2}^{*}+\dot{y}_{2}^{*}-2 \eta^{*} \\
3 y_{2}^{*}+\dot{y}_{2}^{*}-\eta^{*}
\end{array}\right]=\boldsymbol{\alpha}^{-1}\left(y_{1}^{*}, \dot{y}_{1}^{*}, y_{2}^{*}, \dot{y}_{2}^{*}, \eta^{*}\right)
$$

with $\boldsymbol{\alpha}=\left[\alpha_{1}, \alpha_{2}\right]^{\top}$. Thereby, the trajectories $y_{1}^{*}(t), y_{2}^{*}(t)$, and $\eta^{*}(t)$ have to satisfy the BCs (5.12) in order to perform the desired transition between the stationary outputs $\left(y_{1,0}^{*}, y_{2,0}^{*}\right) \rightarrow$ $\left(y_{1, T}^{*}, y_{2, T}^{*}\right)$ in the finite time interval $t \in[0, T]$. The respective $B V P$ (5.14) of the internal dynamics follows from inserting (5.18) in (5.11c):

$$
\begin{aligned}
\dot{\eta}^{*} & =-6 \eta^{*}+\dot{y}_{1}^{*}+9 y_{2}^{*}+3 \dot{y}_{2}^{*} \quad \eta^{*}(0)=\eta_{0}^{*}, \quad \eta^{*}(T)=\eta_{T}^{*} . \\
& =\overline{\boldsymbol{\beta}}\left(\eta^{*}, y_{1}^{*}, \dot{y}_{1}^{*}, y_{2}^{*}, \dot{y}_{2}^{*}\right) .
\end{aligned}
$$

Note that the zero dynamics (with $\dot{y}_{1}^{*}=y_{2}^{*}=\dot{y}_{2}^{*}=0$ ) is stable which shows that the linear MIMO system (5.10) is minimum-phase. The BVP (5.19) is overdetermined with 2 BCs for 1 $O D E$. Hence, one free parameter $p$ is required in the setup functions (5.15) for the outputs $y_{1}^{*}$ and $y_{2}^{*}$ to solve the BVP. Since both outputs $y_{1}^{*}$ and $y_{2}^{*}$ and their time derivatives appear in the internal dynamics, the free parameter $p$ can be provided either in the setup function $\Upsilon_{1}(t, p)$ or $\Upsilon_{2}(t, p)$ by choosing $q_{1}=1, q_{2}=0$ or $q_{1}=0, q_{2}=1$ for the parameter sets in (5.16). Moreover, both functions $\Upsilon_{1}(t, \cdot)$ and $\Upsilon_{2}(t, \cdot)$ have to satisfy the BCs (5.12a) and (5.12b).

The BVP (5.19) with the free parameter $p$ is solved with the MATLAB solver bvp4c. The initial guess for $\eta^{*}\left(t_{k}\right)$ is a linear interpolation between the BCs in (5.19) on a uniform time mesh $t_{k} \in[0, T], k=1, \ldots, 30$. The parameter is initialized with $p=0$. Figure 5.1 shows the nominal trajectories $y_{1}^{*}(t), y_{2}^{*}(t), \eta^{*}(t)$, and the feedforward controls $u_{1}^{*}(t), u_{2}^{*}(t)$ for the setpoint transition $y_{1,0}^{*}=y_{2,0}^{*}=0 \rightarrow y_{1, T}^{*}=y_{2, T}^{*}=1$ in the (dimensionless) time $T=1$. Thereby, the polynomial (2.31a) is used to construct the setup functions $\Upsilon_{1}(t, \cdot)$ and $\Upsilon_{2}(t, \cdot)$. If the free parameter $p$ is assigned to the first setup function, i.e. $q_{1}=1$ and $q_{2}=0$, the first output $y_{1}^{*}(t)=\Upsilon_{1}(t, p)$ shows a large overshoot, whereas the second output $y_{2}^{*}(t)=\Upsilon_{2}(t)$ does not depend on $p$ and therefore is monotonically increasing. For $q_{1}=0$ and $q_{2}=1$, the overshoot appears in the second output $y_{2}^{*}(t)=\Upsilon_{2}(t, p)$. Hence, the assignment of $p$ has to be chosen with respect to the shapes and amplitudes of the trajectories $y_{1}^{*}(t), y_{2}^{*}(t), \eta^{*}(t)$, and the feedforward controls $u_{1}^{*}(t), u_{2}^{*}(t)$. 

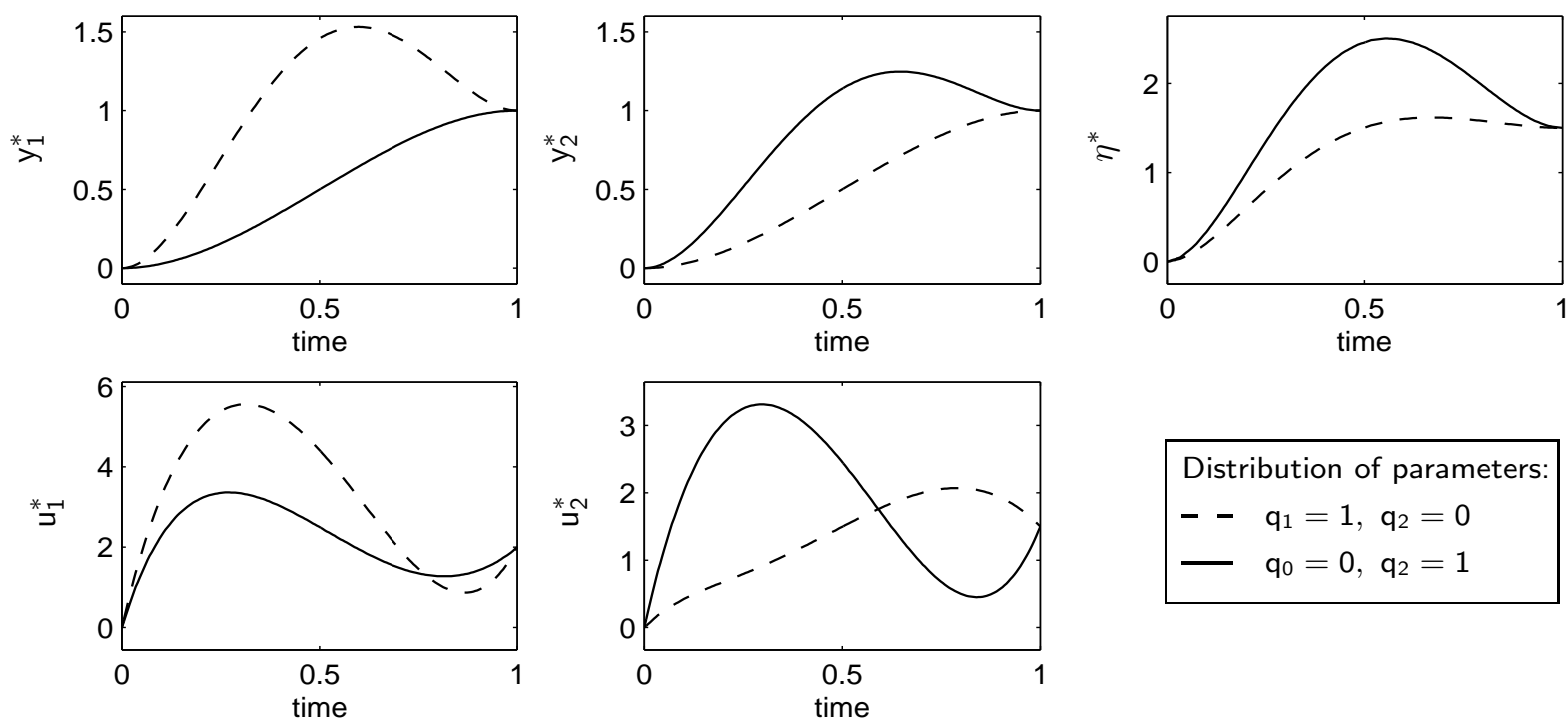

$$
\begin{aligned}
& \text { Distribution of parameters: } \\
& -\quad \mathrm{q}_{1}=1, \mathrm{q}_{2}=0 \\
& -\mathrm{q}_{0}=0, \mathrm{q}_{2}=1
\end{aligned}
$$

Figure 5.1: Setpoint transition $y_{1,0}^{*}=y_{2,0}^{*}=0 \rightarrow y_{1, T}^{*}=y_{2, T}^{*}=1$ of the linear MIMO system (5.10) based on the solution of the BVP (5.19) with different assignments of the free parameter $p$.

\subsection{Incorporation of input constraints}

In this section, the results of Chapter 3 for the incorporation of input constraints are extended to the MIMO case (Graichen and Zeitz, 2006a, 2007). Hence, the feedforward control design has to account for the constraints

$$
u_{k}^{*}(t) \in\left[u_{k}^{-}, u_{k}^{+}\right], \quad k=1, \ldots, m .
$$

on the feedforward trajectories $u_{k}^{*}(t), k=1, \ldots, m$. Following the approach in the SISO case, the input constraints (5.20) are incorporated within the design of the feedforward control (5.13) by parameterizing the highest time derivatives $y_{k}^{*\left(r_{k}\right)}(t), k=1, \ldots, m$ of the outputs. Therefore, $m$ new functions $\hat{\alpha}_{k}=y_{k}^{*\left(r_{k}\right)}$ are introduced which extend the BVP of the internal dynamics (5.14) by the $m$ additional BVPs

$$
\begin{aligned}
& y_{k}^{*\left(r_{k}\right)}=\hat{\alpha}_{k}, \\
& k=1, \ldots, m, \\
& y_{k}^{*}(0)=y_{k, 0}^{*}, \quad y_{k}^{*}(T)=y_{k, T}^{*},\left.\quad y_{k}^{*(i)}\right|_{t=0, T}=0, \quad i=1, \ldots, r_{k}-1 \\
& \dot{\boldsymbol{\eta}}^{*}=\overline{\boldsymbol{\beta}}\left(\boldsymbol{\eta}^{*}, \boldsymbol{y}_{1}^{*}, \hat{\alpha}_{1}, \ldots, \boldsymbol{y}_{m}^{*}, \hat{\alpha}_{m}\right), \quad \boldsymbol{\eta}^{*}(0)=\boldsymbol{\eta}_{0}^{*}, \quad \boldsymbol{\eta}^{*}(T)=\boldsymbol{\eta}_{T}^{*}
\end{aligned}
$$

The solutions $y_{k}^{*}(t)$ and $\boldsymbol{\eta}^{*}(t)$ of the BVPs (5.21)-(5.22) as well as the feedforward trajectories $\boldsymbol{u}^{*}(t)$ in (5.13) depend on the setup of the functions $\hat{\alpha}_{k}=y_{k}^{*\left(r_{k}\right)}$ as described in the following:

(i) If all feedforward controls $u_{k}^{*}(t)$ in (5.13) shall be continuous at the interval bounds $t=0$ and $t=T$, the output trajectories $y_{k}^{*}(t)$ must meet the additional BCs in (5.8) for $\rho_{k}=r_{k}$, which are repeated here for the sake of completeness:

$$
y_{k}^{*\left(r_{k}\right)}(0)=0, \quad y_{k}^{*\left(r_{k}\right)}(T)=0, \quad k=1, \ldots, m .
$$


These BCs have to be satisfied by the functions $\hat{\alpha}_{k}=y_{k}^{*\left(r_{k}\right)}$ if continuity of the feedforward controls $u_{k}^{*}(t)$ is desired.

(ii) The solvability of the BVPs (5.21)-(5.22) defined by $n$ first-order ODEs and $2 n$ BCs requires at least $n$ free parameters. Hence, $m$ setup function $\Phi_{k}\left(t, \boldsymbol{p}_{k}\right), t \in[0, T]$ with the respective free parameter sets

$$
\boldsymbol{p}_{k}=\left(p_{k, 1}, \ldots, p_{k, q_{k}}\right) \quad \text { with } \quad \sum_{k=1}^{m} q_{k}=n
$$

are used to parameterize the functions $\hat{\alpha}_{k}=\Phi_{k}\left(t, \boldsymbol{p}_{k}\right), k=1, \ldots, m$ if the corresponding feedforward control (5.13)

$$
\boldsymbol{u}_{\Phi}^{*}=\boldsymbol{\alpha}^{-1}\left(\boldsymbol{y}_{1}^{*}, \Phi_{1}\left(t, \boldsymbol{p}_{1}\right), \ldots, \boldsymbol{y}_{m}^{*}, \Phi_{m}\left(t, \boldsymbol{p}_{m}\right), \boldsymbol{\eta}^{*}\right)
$$

stays within the constraints $(5.20)$, i.e. $u_{\Phi, j}^{*} \in\left[u_{j}^{-}, u_{j}^{+}\right]$holds for all $j=1, \ldots, m$. The $m$ numbers $q_{k}$ characterize the distribution of the $n$ free parameters over the setup functions $\Phi_{k}\left(t, \boldsymbol{p}_{k}\right)$.

(iii) The functions $\Phi_{k}\left(t, \boldsymbol{p}_{k}\right)$ have to satisfy the BCs $(5.23)$, i.e. $\Phi_{k}\left(0, \boldsymbol{p}_{k}\right)=0$ and $\Phi_{k}\left(T, \boldsymbol{p}_{k}\right)=$ $0, k=1, \ldots, m$, if continuity of the feedforward controls $u_{k}^{*}(t)$ is desired. Thereby, the polynomial or trigonometric series (3.6) introduced in the SISO case can be used to design the functions $\Phi_{k}\left(t, \boldsymbol{p}_{k}\right)$ with or without considering the homogeneous BCs (5.23) for $\Phi_{k}\left(t, \boldsymbol{p}_{k}\right)$.

(iv) If there exists at least one component $u_{\Phi, j}^{*}$ of the feedforward vector (5.25) which violates the constraints, the right-hand sides $\hat{\alpha}_{k}, k=1, \ldots, m$ of (5.21) must be appropriately replanned, such that the respective bounds $\left[u_{j}^{-}, u_{j}^{+}\right]$are met. This is accomplished by the following case-dependent definition of the $m$ functions

$$
\hat{\alpha}_{k}= \begin{cases}\Phi_{k}\left(t, \boldsymbol{p}_{k}\right) & \text { if } u_{\Phi, j}^{*} \in\left[u_{j}^{-}, u_{j}^{+}\right] \\ & \forall j=1, \ldots, m, \quad k=1, \ldots, m \\ \alpha_{k}\left(\boldsymbol{y}_{1}^{*}, \ldots, \boldsymbol{y}_{m}^{*}, \boldsymbol{\eta}^{*}, \hat{\boldsymbol{u}}\right) & \text { else }\end{cases}
$$

with $\hat{\boldsymbol{u}}=\left[\hat{u}_{1}, \ldots, \hat{u}_{m}\right]^{\top}$ and

$$
\hat{u}_{j}=\left\{\begin{array}{ll}
u_{\Phi, j}^{*} & \text { if } u_{\Phi, j}^{*} \in\left[u_{j}^{-}, u_{j}^{+}\right] \\
u_{j}^{-} & \text {if } u_{\Phi, j}^{*}<u_{j}^{-} \\
u_{j}^{+} & \text {if } u_{\Phi, j}^{*}>u_{j}^{+}
\end{array}, \quad j=1, \ldots, m .\right.
$$

If at least one component $u_{\Phi, j}^{*}$ of (5.25) lies outside the respective constraints $\left[u_{j}^{-}, u_{j}^{+}\right]$, all functions $\hat{\alpha}_{k}$ are defined by $\hat{\alpha}_{k}=\alpha_{k}\left(\boldsymbol{y}_{1}^{*}, \ldots, \boldsymbol{y}_{m}^{*}, \boldsymbol{\eta}^{*}, \hat{\boldsymbol{u}}\right)$ with the input vector $\hat{\boldsymbol{u}}$ in (5.27). Thereby, all components $u_{\Phi, j}^{*}$ of $\boldsymbol{u}_{\Phi}^{*}$ which violate the constraints, are set to the respective limit $u_{j}^{-}$or $u_{j}^{+}$. The remaining components $\hat{u}_{j}$ are parameterized by $u_{\Phi, j}^{*}$. Hence, the structure of 
the input-output dynamics (5.6) determines how the outputs $y_{k}^{*\left(r_{k}\right)}=\hat{\alpha}_{k}$ are replanned such that the constraints (5.20) are met.

The case-dependent definition (5.26)-(5.27) of the functions $\hat{\alpha}_{k}$ projects the input constraints (5.20) to constraints on the highest output derivatives $y_{k}^{*\left(r_{k}\right)}$. Thereby, the input constraints (5.20) form a set of admissible inputs $\boldsymbol{u}^{*}=\left[u_{1}^{*}, \ldots, u_{m}^{*}\right]^{\top}$

$$
\mathcal{U}=\left\{\boldsymbol{u}^{*} \in \mathbb{R}^{m} \mid u_{k}^{*} \in\left[u_{k}^{-}, u_{k}^{+}\right], k=1, \ldots, m\right\}
$$

The input-output dynamics (5.6) with $\boldsymbol{\alpha}=\left[\alpha_{1}, \ldots, \alpha_{m}\right]^{\top}$ maps $\mathcal{U}$ to the set

$$
\mathcal{Y}=\left\{\boldsymbol{\alpha}\left(\boldsymbol{y}_{1}^{*}, \ldots, \boldsymbol{y}_{m}^{*}, \boldsymbol{\eta}^{*}, \boldsymbol{u}^{*}\right) \in \mathbb{R}^{m} \mid \boldsymbol{u}^{*} \in \mathcal{U}\right\}
$$

of admissible output derivatives $\left(y_{1}^{*\left(r_{1}\right)}, \ldots, y_{m}^{\left(r_{m}\right)}\right) \in \mathcal{Y}$ with respect to the current output variables $\boldsymbol{y}_{k}^{*}, k=1, \ldots, m$ and internal dynamics state $\boldsymbol{\eta}^{*}$. Figure 5.2 schematically shows the sets $\mathcal{U}$ and $\mathcal{Y}$ for $m=2$ inputs and outputs. ${ }^{5}$ Moreover, the sets $\mathcal{U}$ and $\mathcal{Y}$ can be used to illustrate the concept behind the case-dependent definition (5.26) of $\hat{\alpha}_{k}$. Equation (5.25) is used to calculate the feedforward vector $\boldsymbol{u}_{\Phi}^{*}$ if both outputs $y_{k}^{*\left(r_{k}\right)}=\Phi_{k}\left(t, \boldsymbol{p}_{k}\right), k=1,2$ are parameterized by $\boldsymbol{\Phi}=\left(\Phi_{1}\left(t, \boldsymbol{p}_{1}\right), \Phi_{2}\left(t, \boldsymbol{p}_{2}\right)\right)$. As exemplarily shown in Figure 5.2, $\boldsymbol{u}_{\Phi}^{*}$ lies outside the set $\mathcal{U}$ since the depicted limit $u_{2}^{+}$is violated. Hence, $\boldsymbol{u}_{\Phi}^{*}$ is moved to $\hat{\boldsymbol{u}}$ at the border of the input set $\mathcal{U}$ by means of (5.27), such that the $u_{2}^{*}=u_{2}^{+}$holds. Finally, the functions (5.26b) project the input vector $\hat{\boldsymbol{u}}$ to $\hat{\boldsymbol{\alpha}}=\left(\hat{\alpha}_{1}, \hat{\alpha}_{2}\right) \in \mathcal{Y}$.

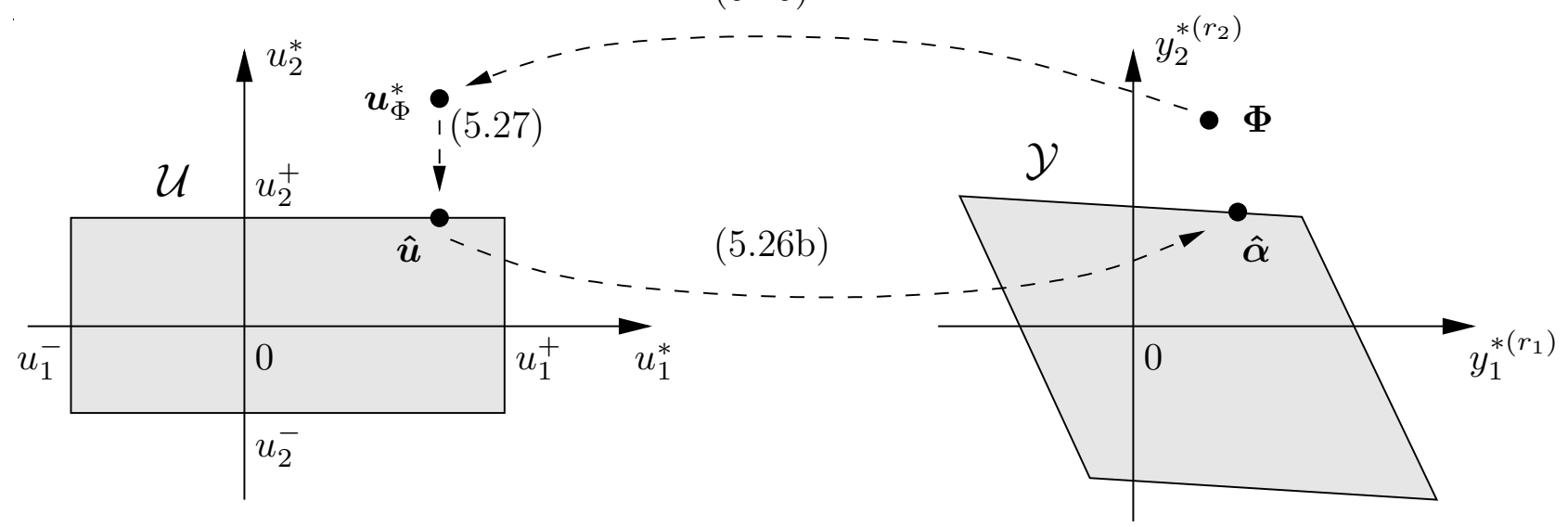

Figure 5.2: Schematic projection of admissible input set $\mathcal{U}$ to output set $\mathcal{Y}(m=2)$ to illustrate the case-dependent function (5.26) with (5.27) and (5.25) in dependence of $\boldsymbol{\Phi}=\left(\Phi_{1}\left(t, \boldsymbol{p}_{1}\right), \Phi_{2}\left(t, \boldsymbol{p}_{2}\right)\right)$.

\footnotetext{
${ }^{5}$ Note that the set $\mathcal{U}$ is compact and the function $\boldsymbol{\alpha}$ of the input-output dynamics (5.6) is continuous due to the assumption that the vector field $\boldsymbol{f}$ and the output functions $h_{k}$ of system (5.1) are sufficiently smooth. This ensures that also the set $\mathcal{Y}$ in (5.29) is compact (Apostol, 1974; Kolmogorov and Fomin, 1975). Moreover, if the input-output dynamics (5.6) is input-affine - which always holds if the original system (5.1) itself is input-affine (Isidori, 1995) - then the function $\boldsymbol{\alpha}\left(\boldsymbol{y}_{1}^{*}, \ldots, \boldsymbol{y}_{m}^{*}, \boldsymbol{\eta}^{*}, \boldsymbol{u}^{*}\right)$ is linear with respect to the input vector $\boldsymbol{u}^{*}$. Hence, the polytopic form of $\mathcal{U}$ is preserved by $\mathcal{Y}$, see e.g. (Boyd and Vandenberghe, 2004) and Figure 5.2.
} 
Remark 5.3. In the case-dependent definition (5.26), the first term (5.26a) for the unconstrained case is also included in the second one (5.26b). If $u_{\Phi, k}^{*} \in\left[u_{k}^{-}, u_{k}^{+}\right]$holds for all $k=1, \ldots, m$, the relation (5.27) yields $\hat{\boldsymbol{u}}=\boldsymbol{u}_{\Phi}^{*}$. Since $\boldsymbol{u}_{\Phi}^{*}$ in (5.25) is based on the inverse input-output dynamics $\boldsymbol{\alpha}^{-1}$, the term (5.26b) becomes $\alpha_{k}\left(\boldsymbol{y}_{1}^{*}, \ldots, \boldsymbol{y}_{m}^{*}, \boldsymbol{\eta}^{*}, \boldsymbol{u}_{\Phi}^{*}\right)=\Phi_{k}\left(t, \boldsymbol{p}_{k}\right)$. The first case (5.26a) is stated explicitly for the sake of clarification and furthermore avoids the calculation of the right-hand sides $\alpha_{k}(\cdot)$ of the input-output dynamics in (5.26b), thus reducing computation time.

An important issue concerns the assignment of the free parameters (5.24). If a certain output $y_{j}^{*}$ with $j \in\{1, \ldots, m\}$ is parameterized by $y_{j}^{*\left(r_{j}\right)}=\hat{\alpha}_{j}=\Phi_{j}\left(t, \boldsymbol{p}_{j}\right)$ for the whole time interval $t \in[0, T]$, then the respective BVP (5.21) reads

$$
y_{j}^{*\left(r_{j}\right)}=\Phi_{j}\left(t, \boldsymbol{p}_{j}\right), \quad y_{j}^{*}(0)=y_{j, 0}^{*}, \quad y_{j}^{*}(T)=y_{j, T}^{*},\left.\quad y_{j}^{*(i)}\right|_{t=0, T}=0, \quad i=1, \ldots, r_{j}-1 .
$$

Hence, in order to satisfy the $2 r_{j}$ BCs in (5.30), the free parameter set $\boldsymbol{p}_{j}=\left(p_{j, 1}, \ldots, p_{j, q_{j}}\right)$ in the setup function $\Phi_{j}\left(t, \boldsymbol{p}_{j}\right)$ must contain at least $r_{j}$ elements to provide sufficient free parameters for the solvability of the decoupled BVP (5.30). This consideration is generalized to all $m$ output BVPs (5.21) by the condition

$$
q_{k} \geq r_{k}, \quad k=1, \ldots, m \quad \text { with } \quad \sum_{k=1}^{m} r_{k}=r \leq n,
$$

which holds in addition to (5.24). The condition (5.31) also covers the case that all inputs $u_{\Phi, k}^{*}, k=1, \ldots, m$ in (5.25) stay inside their constraints $\left[u_{k}^{-}, u_{k}^{+}\right]$for the whole time interval $t \in[0, T]$, such that all output BVPs (5.21) are decoupled according to (5.30) with $j=1, \ldots, m$. Note that in contrast to (5.16), the condition (5.31) requires that $\boldsymbol{p}_{k}$ contains at least one element, because $r_{k} \geq 1$ holds for the vector relative degree $\left\{r_{1}, \ldots, r_{m}\right\}$ since the output functions $y_{k}=h_{k}(\boldsymbol{x})$ in (5.1) are independent of the input $\boldsymbol{u}$, cf. Definition 5.1. Nevertheless, (5.31) shows that some freedom is left concerning the distribution of the remaining $n-r$ parameters over the parameter sets $\boldsymbol{p}_{k}$.

Remark 5.4. In the special case that the outputs $y_{k}, k=1, \ldots, m$ represent flat outputs (Fliess et al., 1995), $\sum_{k=1}^{m} r_{k}=n$ holds and no internal dynamics (5.22) exists. Hence, the distribution (5.31) of the free parameters is uniquely determined by $q_{k}=r_{k}, k=1, \ldots, m$.

Example 5.3 (Example 5.2 continued). In order to incorporate the input constraints (5.20) within the feedforward control design for the linear MIMO system in Example 5.2, the internal dynamics BVP (5.19) is extended by $m=2$ ODEs (5.21) with the vector relative degree $\{1,1\}$ :

$$
\begin{array}{rlrl}
\dot{y}_{1}^{*} & =\hat{\alpha}_{1}, & y_{1}^{*}(0)=y_{1,0}^{*}, & y_{1}^{*}(T)=y_{1, T}^{*} \\
\dot{y}_{2}^{*}=\hat{\alpha}_{2}, & y_{2}^{*}(0)=y_{2,0}^{*}, & y_{2}^{*}(T)=y_{2, T}^{*} \\
\dot{\eta}^{*}=-6 \eta^{*}+\hat{\alpha}_{1}+9 y_{2}^{*}+3 \hat{\alpha}_{2}, & \eta^{*}(0)=\eta_{0}^{*}, & & \eta^{*}(T)=\eta_{T}^{*} . \\
& =\overline{\boldsymbol{\beta}}\left(\eta^{*}, y_{1}^{*}, \hat{\alpha}_{1}, y_{2}^{*}, \hat{\alpha}_{2}\right) . & &
\end{array}
$$


The BVPs (5.32a)-(5.32c) are overdetermined by $2 n=6$ BCs for $n=3$ ODEs. Hence, $n=3$ free parameters are assigned to the setup functions $\Phi_{1}\left(t, p_{1}\right)$ and $\Phi_{2}\left(t, \boldsymbol{p}_{2}\right)$ with $q_{1}=1$ and $q_{2}=2$, i.e. $\boldsymbol{p}_{2}=\left(p_{2,1}, p_{2,2}\right){ }^{6}$ The sine series (3.6d) with homogeneous $B C s$ is used to construct the setup functions $\Phi_{1}\left(t, p_{1}\right)$ and $\Phi_{2}\left(t, \boldsymbol{p}_{2}\right)$ in order to ensure continuity of the feedforward control (5.18) at $t=0$ and $t=T$. The case-dependent definition of $\hat{\alpha}_{1}$ and $\hat{\alpha}_{2}$ follows from (5.26) -(5.27) with the input-output dynamics (5.11a)-(5.11b) and the feedforward control vector (5.18). The initial guess of $y_{1}^{*}\left(t_{k}\right), y_{2}^{*}\left(t_{k}\right)$, and $\eta^{*}\left(t_{k}\right)$ for the bvp4c-solution is a linear interpolation between the respective $B C s$ on a uniform time mesh $t_{k} \in[0, T], k=$ $1, \ldots, 30$. The free parameters are initialized with $p_{1}=0$ and $\boldsymbol{p}_{2}=\left(p_{2,1}, p_{2,2}\right)=\mathbf{0}$.
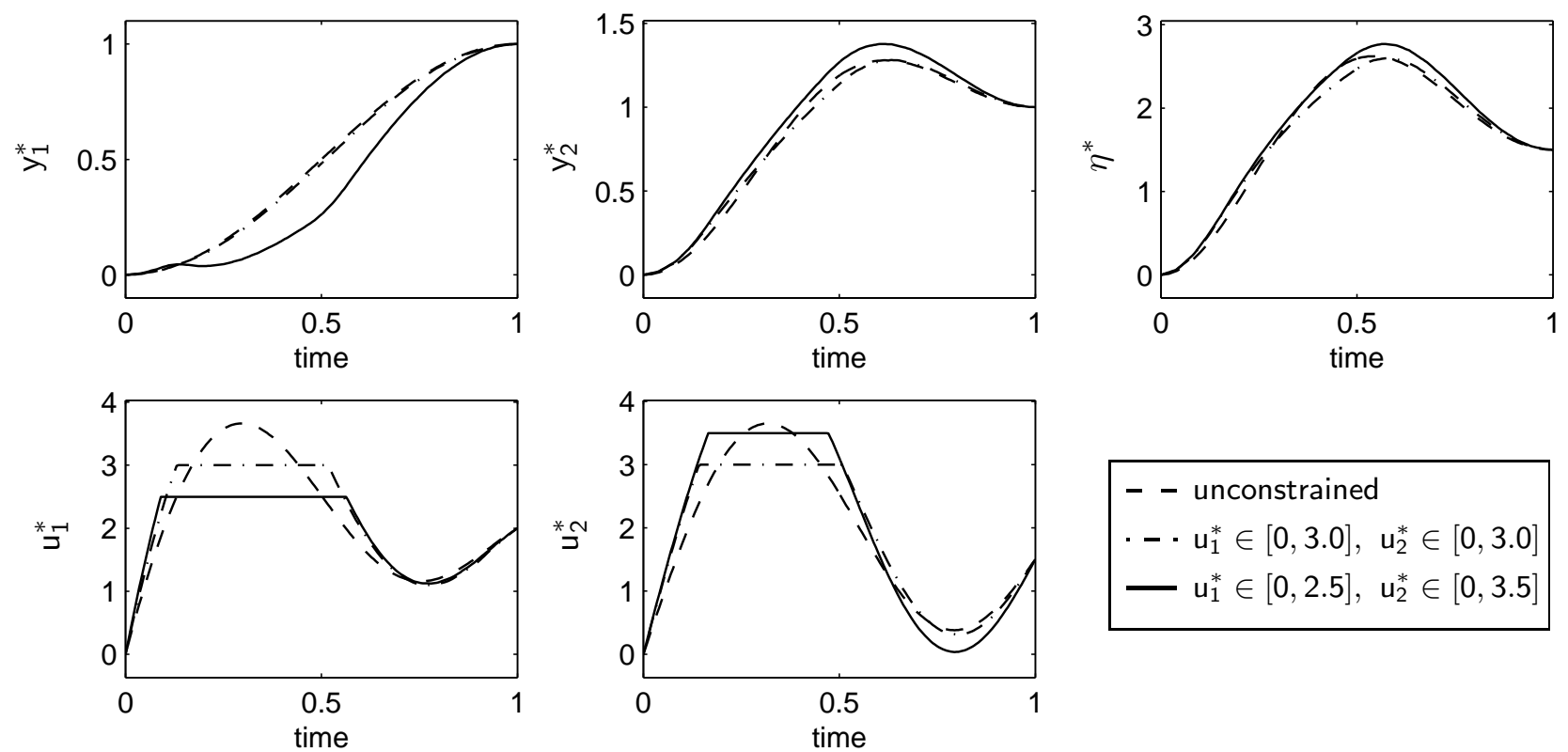

Figure 5.3: Setpoint transition $y_{1,0}^{*}=y_{2,0}^{*}=0 \rightarrow y_{1, T}^{*}=y_{2, T}^{*}=1$ of the linear MIMO system (5.10) based on the solution of the BVPs (5.32) with different input constraints.
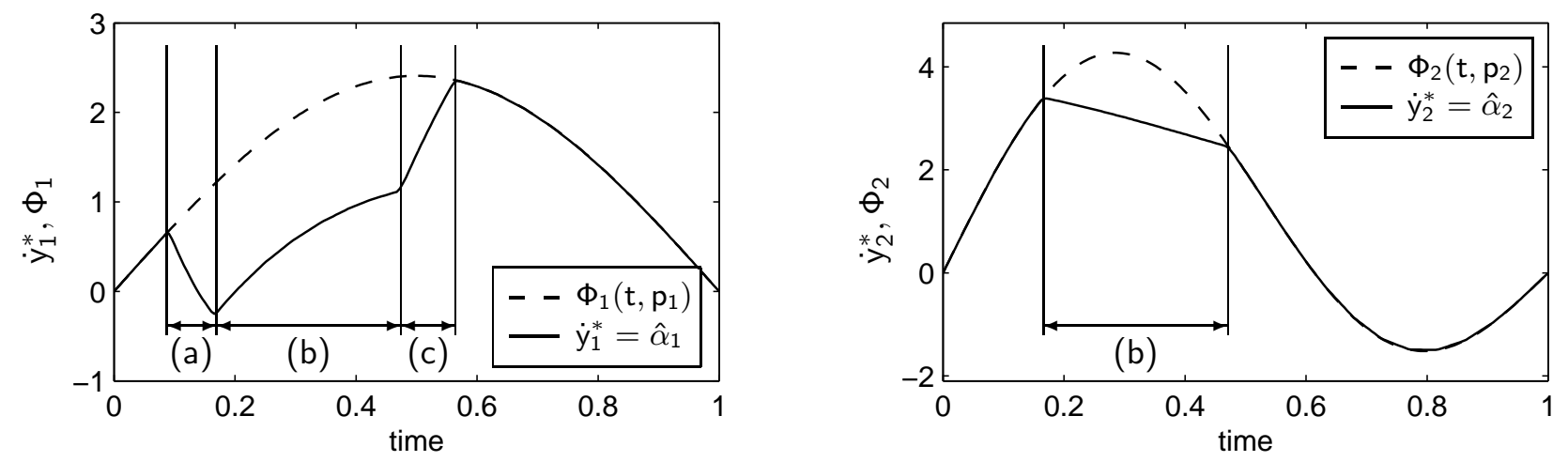

Figure 5.4: Output derivatives $\dot{y}_{k}^{*}=\hat{\alpha}_{k}, k=1,2$ and the respective setup functions $\Phi_{1}\left(t, p_{1}\right), \Phi_{2}\left(t, \boldsymbol{p}_{2}\right)$ corresponding to the transition in Figure 5.3 with the input constraints $u_{1}^{*} \in[0,2.5]$ and $u_{2}^{*} \in[0,3.5]$.

\footnotetext{
${ }^{6}$ Note that due to condition (5.31), $r_{1}=r_{2}=1$ free parameter is required in each setup function. Hence, the third free parameter is assigned to the second function $\Phi_{2}\left(t, \boldsymbol{p}_{2}\right)$, which corresponds to the choice $q_{1}=0$ and $q_{2}=1$ in the unconstrained case, see Example 5.2 and Figure 5.1.
} 
Figure 5.3 shows the nominal trajectories $y_{1}^{*}(t), y_{2}^{*}(t), \eta^{*}(t)$, and the feedforward controls $u_{1}^{*}(t)$, $u_{2}^{*}(t)$ for the same transition scenario as in Figure 5.1. The unconstrained trajectories $(--)$ are obtained by setting the constraints for $u_{k}^{*} \in\left[u_{k}^{-}, u_{k}^{+}\right]$to sufficiently large values. The trajectories (-.) and (-) correspond to two different input constraints and are clearly replanned compared to the unconstrained case. In particular for the input constraints $u_{1}^{*} \in[0,2.5]$ and $u_{2}^{*} \in[0,3.5]$, the feedforward controls $u_{1}^{*}(t)$ and $u_{2}^{*}(t)$ touch the upper constraints $u_{1}^{+}=2.5$ and $u_{2}^{+}=3.5$ in two overlapping time intervals. The corresponding output derivatives $\dot{y}_{1}^{*}=\hat{\alpha}_{1}$ and $\dot{y}_{2}^{*}=\hat{\alpha}_{2}$ are shown in Figure 5.4 together with the setup functions $\Phi_{1}\left(t, p_{1}\right)$ and $\Phi_{2}\left(t, \boldsymbol{p}_{2}\right)$. In the first and last time intervals (a) and (c), only $u_{1}^{*}(t)$ touches the constraint $u_{1}^{+}=2.5$ (see Figure 5.3). Hence, $\dot{y}_{1}^{*}=\hat{\alpha}_{1}$ and $\dot{y}_{2}^{*}=\hat{\alpha}_{2}$ are determined by (5.26b) with the input $\hat{\boldsymbol{u}}=\left[u_{1}^{+}, u_{\Phi, 2}^{*}\right]^{\top}$ following from (5.27). Since only the function $\alpha_{1}$ of the input-output dynamics (5.11a)-(5.11b) depends on $u_{1}$, only $\dot{y}_{1}^{*}$ is replanned by $\hat{\alpha}_{1}=\alpha_{1}\left(y_{1}^{*}, \eta^{*}, u_{1}^{+}, u_{\Phi, 2}^{*}\right)$, whereas the second function $\hat{\alpha}_{2}=$ $\alpha_{2}\left(y_{2}^{*}, \eta^{*}, u_{\Phi, 2}^{*}\right)=\Phi_{2}\left(t, \boldsymbol{p}_{2}\right)$ is exactly the setup function $\Phi_{2}\left(t, \boldsymbol{p}_{2}\right)$. In the time interval (b) when also the second input $u_{2}^{*}$ touches the constraint $u_{2}^{+}=3.5$, both $\dot{y}_{1}^{*}=\hat{\alpha}_{1}$ and $\dot{y}_{2}^{*}=\hat{\alpha}_{2}$ are defined by $\hat{\alpha}_{1}=\alpha_{1}\left(y_{1}^{*}, \eta^{*}, u_{1}^{+}, u_{2}^{+}\right)$and $\hat{\alpha}_{2}=\alpha_{2}\left(y_{2}^{*}, \eta^{*}, u_{2}^{+}\right)$, see Figure 5.4 .

\subsection{Incorporation of output constraints}

Similar to the incorporation of input constraints described in the last section, the output constraints (4.1) addressed in Chapter 4 can be extended to the MIMO case by constraining each output trajectory

$$
y_{k}^{*(i)}(t) \in\left[y_{k, i}^{-}, y_{k, i}^{+}\right], \quad k=1, \ldots, m, \quad i=0,1, \ldots, r_{k} .
$$

Moreover, the condition (4.2) is adapted to the MIMO case by demanding that the constraints (5.33) satisfy the inequality

$$
y_{k, i}^{-}<0<y_{k, i}^{+}, \quad k=1, \ldots, m, \quad i=1, \ldots, r_{k} .
$$

\subsubsection{System representation for constrained outputs}

According to Section 4.1, the output constraints (5.33) for each output $y_{k}^{*}$ are incorporated in a new system (4.9) by introducing saturation functions and successively differentiating the output $y_{k}^{*}$ :

$$
\left.\begin{array}{rl}
y_{k}^{*} & =\psi_{1}^{k}\left(\xi_{1}^{k}, \psi_{1}^{k \pm}\right) \\
\dot{\xi}_{1}^{k} & =\psi_{2}^{k}\left(\xi_{2}^{k}, \psi_{2}^{k \pm}\left(\xi_{1}^{k}\right)\right) \\
\dot{\xi}_{2}^{k} & =\psi_{3}^{k}\left(\xi_{3}^{k}, \psi_{3}^{k \pm}\left(\boldsymbol{\xi}_{2}^{k}\right)\right) \\
\vdots & \vdots \\
\dot{\xi}_{r_{k}}^{k} & =\psi_{r_{k}+1}^{k}\left(v_{k}, \psi_{r_{k}+1}^{k \pm}\left(\boldsymbol{\xi}_{r_{k}}^{k}\right)\right)
\end{array}\right\} \quad \dot{\boldsymbol{\xi}}_{r_{k}}^{k}=\boldsymbol{\psi}_{r_{k}+1}^{k}\left(\boldsymbol{\xi}_{r_{k}}^{k}, v_{k}\right), \quad k=1, \ldots, m
$$


Hence, each output (5.35) is described by a system (5.36) with the state $\boldsymbol{\xi}_{r_{k}}^{k}=\left[\xi_{1}^{k}, \ldots, \xi_{r_{k}}^{k}\right]^{\top}$, the vector field $\boldsymbol{\psi}_{r_{k}+1}^{k}=\left[\psi_{2}^{k}, \ldots, \psi_{r_{k}+1}^{k}\right]^{\top}$, and a new input $v_{k}$. In analogy to the SISO case, the saturation functions $\psi_{1}^{k}\left(\xi_{1}^{k}, \psi_{1}^{k \pm}\right)$ and $\psi_{i}^{k}\left(\xi_{i}^{k}, \psi_{i}^{k \pm}\left(\boldsymbol{\xi}_{i-1}^{k}\right)\right), i=2, \ldots, r_{k}$ are constructed by the smooth function (4.12), whereas the last saturation functions $\psi_{r_{k}+1}^{k}\left(v_{k}, \psi_{r_{k}+1}^{k \pm}\left(\boldsymbol{\xi}_{r_{k}}^{k}\right)\right)$ are defined by the ramp-shaped function (4.13), as depicted in Figure 4.1 and 4.2.

The $i$-th time derivative $y_{k}^{*(i)}$ of the output (5.35) is determined according to Equation (4.14) in Lemma 4.1:

$$
\begin{aligned}
y_{k}^{*(i)} & =L_{\boldsymbol{\psi}_{r_{k}+1}^{k}}^{i} \psi_{1}^{k}\left(\xi_{1}^{k}, \psi_{1}^{k \pm}\right) \\
& =\gamma_{i}^{k}\left(\boldsymbol{\xi}_{i}^{k}\right)+\prod_{j=1}^{i} \frac{\partial \psi_{j}^{k}}{\partial \xi_{j}^{k}} \cdot \psi_{i+1}^{k}\left(\cdot, \psi_{i+1}^{k \pm}\left(\boldsymbol{\xi}_{i}^{k}\right)\right), \quad k=1, \ldots, m, \quad i=1, \ldots, r_{k}
\end{aligned}
$$

whereby $L_{\boldsymbol{\psi}_{r_{k}+1}^{k}}$ denotes the Lie derivative along the vector field $\boldsymbol{\psi}_{r_{k}+1}^{k}$ in (5.36). The argument $(\cdot)$ in (5.37) is given by $\xi_{i+1}^{k}$ or $v_{k}$ for $i=r_{k}$, respectively. The remaining nonlinear terms are comprised in $\gamma_{i}^{k}\left(\boldsymbol{\xi}_{i}^{k}\right)$.

The limits of the saturation functions in (5.35)-(5.36) are determined according to (4.17):

$$
\psi_{1}^{k \pm}=y_{k, 0}^{ \pm}, \quad \psi_{i+1}^{k \pm}\left(\boldsymbol{\xi}_{i}^{k}\right)=\frac{y_{k, i}^{ \pm}-\gamma_{i}^{k}\left(\boldsymbol{\xi}_{i}^{k}\right)}{\prod_{j=1}^{i} \frac{\partial \psi_{j}^{k}}{\partial \xi_{j}^{k}}}, \quad k=1, \ldots, m, \quad i=1, \ldots, r_{k},
$$

in order to ensure that the output constraints (5.33) are satisfied. The $m$ systems (5.36) can be constructed separately by successively differentiating the outputs $y_{k}^{*}$ to obtain the nonlinear terms $\gamma_{i}^{k}\left(\boldsymbol{\xi}_{i}^{k}\right)$ in (5.37), which are required to calculate the saturation limits (5.37). As pointed out in Section 4.1.3, these analytic operations can be performed with computer-algebra-systems like Mathematica or MAPLE.

\subsubsection{Boundary value problems for constrained outputs}

The output constraints (5.33) are incorporated within the MIMO feedforward control design by substituting the $m$ output BVPs (5.21) by the new systems (5.36):

$$
\begin{array}{rlrl}
\dot{\boldsymbol{\xi}}_{r_{k}}^{k} & =\boldsymbol{\psi}_{r_{k}+1}^{k}\left(\boldsymbol{\xi}_{r_{k}}, v_{k}\right), & \boldsymbol{\xi}_{r_{k}}^{k}(0)=\boldsymbol{\xi}_{r_{k}, 0}^{k}, & \boldsymbol{\xi}_{r_{k}}^{k}(T)=\boldsymbol{\xi}_{r_{k}, T}^{k}, \quad k=1, \ldots, m \\
\dot{\boldsymbol{\eta}}^{*}=\overline{\boldsymbol{\beta}}\left(\boldsymbol{\eta}^{*}, \boldsymbol{y}_{1}^{*}, y_{1}^{*\left(r_{1}\right)}, \ldots, \boldsymbol{y}_{m}^{*}, y_{m}^{*\left(r_{m}\right)}\right), & \boldsymbol{\eta}^{*}(0)=\boldsymbol{\eta}_{0}^{*}, & \boldsymbol{\eta}^{*}(T)=\boldsymbol{\eta}_{T}^{*}
\end{array}
$$

The outputs $y_{k}^{*}$ and their time derivatives $y_{k}^{*(i)}, i=1, \ldots, r_{k}$ appearing in the internal dynamics (5.40) are determined by the relations (5.35) and (5.37) in dependence of the states $\boldsymbol{\xi}_{r_{k}}^{k}$. This leads to a one-side coupling of the BVPs (5.39)-(5.40).

As described in detail in Section 4.2.1, the BCs of the outputs $y_{k}^{*}$ in (5.21) can be converted to the boundary values $\boldsymbol{\xi}_{r_{k}, 0}^{k}$ and $\boldsymbol{\xi}_{r_{k}, T}^{k}$ for the states $\boldsymbol{\xi}_{r_{k}}^{k}=\left[\xi_{1}^{k}, \ldots, \xi_{r_{k}}^{k}\right]^{\top}$ according to Equation 
(4.20) and (4.24):

$$
\begin{array}{lll}
\xi_{1,0}^{k}=\psi_{1}^{k^{-1}}\left(y_{k, 0}^{*}, \psi_{1}^{k \pm}\right), & \xi_{1, T}^{k}=\psi_{1}^{k^{-1}}\left(y_{k, T}^{*}, \psi_{1}^{k \pm}\right), & k=1, \ldots, m, \\
\xi_{i, 0}^{k}=\psi_{i}^{k^{-1}}\left(0, \psi_{i}^{k \pm}\left(\boldsymbol{\xi}_{i-1,0}^{k}\right)\right), & \xi_{i, T}^{k}=\psi_{i}^{k^{-1}}\left(0, \psi_{i}^{k \pm}\left(\boldsymbol{\xi}_{i-1, T}^{k}\right)\right), & i=2, \ldots, r_{k} .
\end{array}
$$

The boundary values (5.41) are easily calculated by inverting the saturation function (4.12). The remaining boundary values (5.42) have to be determined successively as they depend on the previously calculated values $\boldsymbol{\xi}_{i-1,0}^{k}$ and $\boldsymbol{\xi}_{i-1, T}^{k}$. Moreover, as shown in Section 4.2 .2 for the SISO case, if a certain output $y_{k}^{*}$ has symmetric constraints (5.35) on its time derivatives, i.e.

$$
y_{k, i}^{-}=-y_{k, i}^{+}, \quad i=1, \ldots, r_{k}-1
$$

holds, then the boundary values (5.42) simplify to

$$
\xi_{i, 0}^{k}=\xi_{i, T}^{k}=0, \quad i=2, \ldots, r_{k} .
$$

Hence, calculating the BCs for the respective BVP (5.39) reduces to the solution of (5.41), since the remaining $\mathrm{BCs}$ for the states $\xi_{i}^{k}, i=1, \ldots, r_{k}$ are homogeneous.

The solution of the BVPs (5.39)-(5.40) are the trajectories $\boldsymbol{\xi}_{r_{k}}^{k}(t)=\left[\xi_{1}^{k}(t), \ldots, \xi_{r_{k}}^{k}(t)\right]^{\top}$ and $\boldsymbol{\eta}^{*}(t), t \in[0, T]$ which depend on the design of the new inputs $v_{k}$ with respect to the following points:

(i) As mentioned in point (i) of Section 5.2, all output trajectories $y_{k}^{*}(t)$ have to satisfy the additional BCs (5.23), if continuity of the feedforward control vector (5.13) at $t=0$ and $t=T$ is desired. This leads to the additional BCs

$$
v_{k}(0)=0, \quad v_{k}(T)=0, \quad k=1, \ldots, m
$$

for the new inputs $v_{k}(t)$, cf. Section 4.2.3.

(ii) The $m+1$ BVPs (5.39)-(5.40) are overdetermined by $2 n$ BCs for $n$ ODEs. Similar to Section $5.2, n$ free parameters are provided in $m$ setup functions for the new inputs

$$
v_{k}=\Phi_{k}\left(t, \boldsymbol{p}_{k}\right), \quad k=1, \ldots, m
$$

whereby the parameter sets $\boldsymbol{p}_{k}$ in (5.24) have to satisfy the inequality (5.31) (see Section 5.2 for details). Hence, some freedom exists to assign the remaining $n-r$ free parameters over the setup functions $\Phi_{k}\left(t, \boldsymbol{p}_{k}\right)$. In view of (5.45), the functions $\Phi_{k}\left(t, \boldsymbol{p}_{k}\right)$ have homogeneous BCs $\Phi_{k}\left(0, \boldsymbol{p}_{k}\right)=\Phi_{k}\left(T, \boldsymbol{p}_{k}\right)=0$ if continuity of the feedforward control vector $\boldsymbol{u}^{*}(t)$ is desired. Thereby, the polynomial or trigonometric series (3.6) as introduced in the SISO case can be used to design the functions $\Phi_{k}\left(t, \boldsymbol{p}_{k}\right)$ with respect to the desired continuity property of $\boldsymbol{u}^{*}(t)$.

The solution of the $m+1$ BVPs (5.39)-(5.40) with the free parameter sets $\boldsymbol{p}_{k}$ in the setup functions (5.46) comprises the trajectories $\boldsymbol{\xi}_{r_{k}}^{k}(t), k=1, \ldots, m$ and $\boldsymbol{\eta}^{*}(t), t \in[0, T]$. The constrained output trajectories $y_{k}^{*(i)}(t), i=0,1, \ldots, r_{k}$ as well as the feedforward controls $u_{k}^{*}(t), k=1, \ldots, m$ finally follow from (5.35), (5.37), and (5.13). 
Example 5.4 (Example 5.3 continued). The output constraints (5.33) are incorporated in the feedforward control design of the linear MIMO system (5.10) by replacing the output BVPs (5.32a) $-(5.32 \mathrm{~b})$ by $m=2$ BVPs (5.39) for the coordinates $\xi^{1}$ and $\xi^{2}$ :

$$
\begin{aligned}
& \dot{\xi}^{1}=\psi_{2}^{1}\left(v_{1}, \psi_{2}^{1 \pm}\left(\xi^{1}\right)\right), \\
& \xi^{1}(0)=\xi_{0}^{1}, \quad \xi^{1}(T)=\xi_{T}^{1}, \\
& \dot{\xi}^{2}=\psi_{2}^{2}\left(v_{2}, \psi_{2}^{2 \pm}\left(\xi^{2}\right)\right), \\
& \xi^{2}(0)=\xi_{0}^{2}, \quad \xi^{2}(T)=\xi_{T}^{2}, \\
& \dot{\eta}^{*}=-6 \eta^{*}+\dot{y}_{1}^{*}+9 y_{2}^{*}+3 \dot{y}_{2}^{*}, \\
& \eta^{*}(0)=\eta_{0}^{*}, \\
& \eta^{*}(T)=\eta_{T}^{*} . \\
& =\overline{\boldsymbol{\beta}}\left(\eta^{*}, y_{1}^{*}, \dot{y}_{1}^{*}, y_{2}^{*}, \dot{y}_{2}^{*}\right) .
\end{aligned}
$$

Thereby, the output variables $y_{1}^{*}, \dot{y}_{1}^{*}$ and $y_{2}^{*}, \dot{y}_{2}^{*}$ appearing in the internal dynamics $(5.47 \mathrm{c})$ are expressed by the relations (5.35) and (5.37) (also see (4.30) of Example 4.1, respectively)

$$
y_{k}^{*}=\psi_{1}^{k}\left(\xi^{k}, \psi_{1}^{k \pm}\right), \quad \dot{y}_{k}^{*}=\frac{\partial \psi_{1}^{k}}{\partial \xi^{k}} \psi_{2}^{k}\left(v_{k}, \psi_{2}^{k \pm}\left(\xi^{k}\right)\right), \quad k=1,2
$$

in terms of the coordinates $\xi^{1}, \xi^{2}$, and the new inputs $v_{1}$ and $v_{2}$. The saturation functions $\psi_{1}^{k}\left(\xi^{k}, \psi_{1}^{k \pm}\right)$ and $\psi_{2}^{k}\left(v_{k}, \psi_{2}^{k \pm}\left(\xi^{k}\right)\right)$ are defined according to (4.12) and (4.13) with the respective limits $\psi_{1}^{k \pm}$ and $\psi_{2}^{k \pm}\left(\xi^{k}\right)$ in (5.38). The boundary values $\xi_{0}^{k}$ and $\xi_{T}^{k}$ in $(5.47 \mathrm{a})-(5.47 \mathrm{~b})$ are given by (5.41).

The solvability of the overdetermined BVPs (5.47) requires $n=3$ free parameters. In accordance with Example 5.3, the parameters are assigned to the setup functions $\Phi_{1}\left(t, p_{1}\right)$ and $\Phi_{2}\left(t, \boldsymbol{p}_{2}\right)$ with $q_{1}=1$ and $q_{2}=2$, and the sine series (3.6d) is used to construct both $\Phi_{1}\left(t, p_{1}\right)$ and $\Phi_{2}\left(t, \boldsymbol{p}_{2}\right)$. The initial guess for the bvp4c-solution of (5.47)-(5.48) is again a linear interpolation between the respective $B C$ s on a uniform time mesh with 30 points and the parameter values $p_{1}=0$ and $\boldsymbol{p}_{2}=\left(p_{2,1}, p_{2,2}\right)=\mathbf{0}$. Figure 5.5 shows the resulting nominal trajectories of the outputs (5.48), $\eta^{*}(t)$, and the feedforward controls (5.18) for different output constraints. The unconstrained trajectories (- -) are obtained by setting the values of $y_{k, i}^{ \pm}, i, k=1,2$ to sufficiently large values. For the constrained trajectories (- - ) and (-), the overshoot in the output $y_{2}^{*}(t)$ is clearly reduced while at the same time the amplitude of the derivative $\dot{y}_{2}^{*}(t)$ is constrained. Especially for $y_{2}^{*} \in[-0.2,1.2]$, the output $y_{2}^{*}(t)$ stays at the limit $y_{2}^{+}=1.2$ with an aggressive behavior of the derivative $\dot{y}_{2}^{*} \in[-5,5]$. The constraints on the first output $y_{1}^{*}(t)$ have no effect, since $y_{1}^{*}(t)$ is monotonically increasing. However, limiting the velocity $\dot{y}_{1}^{*}$ results in a ramp-like profile of $y_{1}^{*}(t)$.

\subsection{Incorporation of input and output constraints}

The input and output constraints (5.20)-(5.33) can be treated simultaneously in the feedforward control design by extending the results of Section 4.3 to the MIMO case. Hence, in addition to first $r_{k}$ constraints $y_{k}^{*(i)} \in\left[y_{i, k}^{-}, y_{i, k}^{+}\right], i=0,1, \ldots, r_{k}-1$ for each output $y_{k}^{*}, k=1, \ldots, m$, the input constraints $(5.20)$ can be projected to constraints on the highest output derivatives $y_{k}^{*\left(r_{k}\right)}$ according to the definition of $\hat{\alpha}_{k}$ in $(5.26)-(5.27)$. 

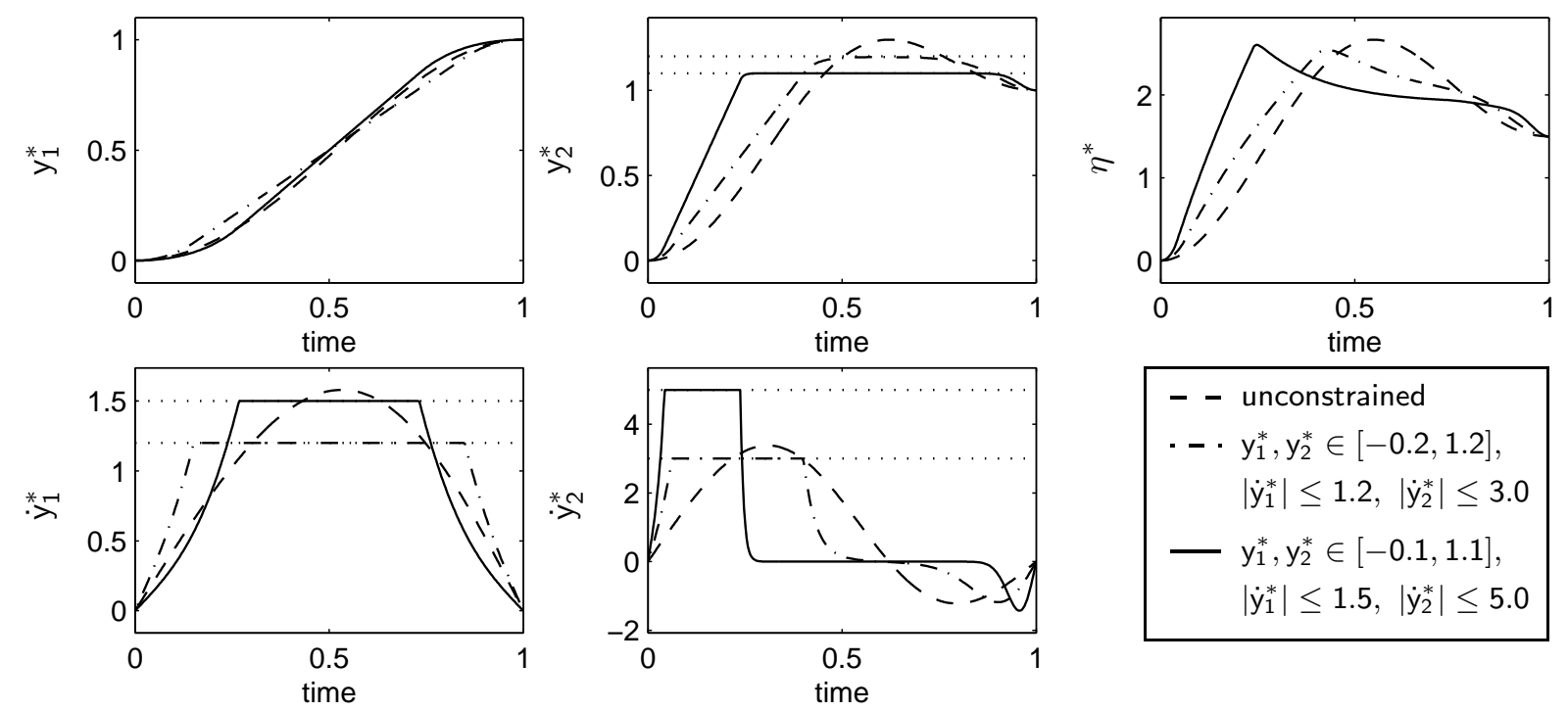

$$
\begin{aligned}
- & \text { unconstrained } \\
\cdots & \mathrm{y}_{1}^{*}, \mathrm{y}_{2}^{*} \in[-0.2,1.2], \\
& \left|\dot{\mathrm{y}}_{1}^{*}\right| \leq 1.2,\left|\dot{\mathrm{y}}_{2}^{*}\right| \leq 3.0 \\
- & \mathrm{y}_{1}^{*}, \mathrm{y}_{2}^{*} \in[-0.1,1.1], \\
& \left|\dot{y}_{1}^{*}\right| \leq 1.5,\left|\dot{y}_{2}^{*}\right| \leq 5.0
\end{aligned}
$$
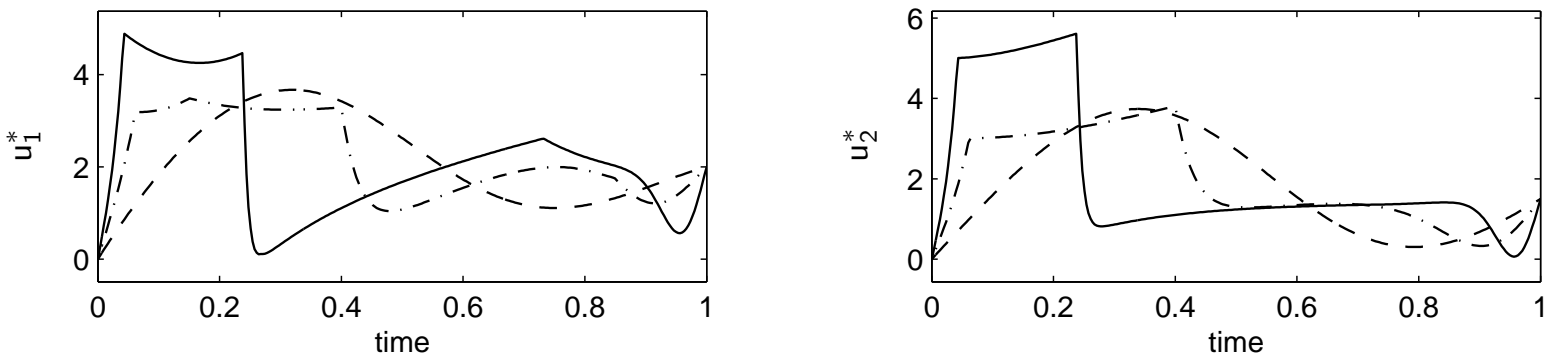

Figure 5.5: Setpoint transition $y_{1,0}^{*}=y_{2,0}^{*}=0 \rightarrow y_{1, T}^{*}=y_{2, T}^{*}=1$ of the linear MIMO system (5.10) based on the solution of the BVPs (5.47) with different output constraints.

Therefore, the final saturation functions $\psi_{r_{k}+1}^{k}\left(v_{k}, \psi_{r_{k}+1}^{k \pm}\right)$ are replaced by new functions $\widehat{\psi}_{r_{k}+1}^{k}$ for the highest output derivatives (5.37), i.e.

$$
y_{k}^{*\left(r_{k}\right)}=\gamma_{r_{k}}^{k}\left(\boldsymbol{\xi}_{r_{k}}^{k}\right)+\prod_{j=1}^{r_{k}} \frac{\partial \psi_{j}^{k}}{\partial \xi_{j}^{k}} \cdot \widehat{\psi}_{r_{k}+1}^{k}, \quad k=1, \ldots, m,
$$

whereby the functions $\widehat{\psi}_{r_{k}+1}^{k}$ have to be defined according to (5.26)-(5.27) in order to account for the input constraints (5.20). Hence, the new inputs $v_{k}$ are used to parameterize $\widehat{\psi}_{r_{k}+1}^{k}=$ $v_{k}, k=1, \ldots, m$ if the corresponding feedforward vector $(5.13)$

$$
\boldsymbol{u}_{v}^{*}=\boldsymbol{\alpha}^{-1}\left(\boldsymbol{y}_{1}^{*}, y_{1, v}^{*\left(r_{1}\right)}, \ldots, \boldsymbol{y}_{m}, y_{m, v}^{*\left(r_{m}\right)}, \boldsymbol{\eta}^{*}\right)
$$

with

$$
y_{k, v}^{*\left(r_{k}\right)}=\gamma_{r_{k}}^{k}\left(\boldsymbol{\xi}_{r_{k}}^{k}\right)+\prod_{j=1}^{r_{k}} \frac{\partial \psi_{j}^{k}}{\partial \xi_{j}^{k}} \cdot v_{k}, \quad k=1, \ldots, m
$$

stays within the constraints (5.20), i.e. $u_{v, j}^{*} \in\left[u_{j}^{-}, u_{j}^{+}\right]$holds for all $j=1, \ldots, m$. The respective output derivatives $y_{k, v}^{*\left(r_{k}\right)}$ follow from (5.49) with $\widehat{\psi}_{r_{k}+1}^{k}=v_{k}, k=1, \ldots, m$. However, if at least one component $u_{v, j}^{*}$ of (5.50) violates the constraints, then according to (5.26), $y_{k}^{*\left(r_{k}\right)}$ in (5.49) must be set to $y_{k}^{*\left(r_{k}\right)} \stackrel{!}{=} \alpha_{k}\left(\boldsymbol{y}_{1}^{*}, \ldots, \boldsymbol{y}_{m}^{*}, \boldsymbol{\eta}^{*}, \hat{\boldsymbol{u}}\right)$ such that the feedforward control (5.13) becomes 
$\boldsymbol{u}^{*}=\hat{\boldsymbol{u}}$. Thereby, the input vector $\hat{\boldsymbol{u}}=\left[\hat{u}_{1}, \ldots, \hat{u}_{m}\right]^{\top}$ is defined similar to (5.27)

$$
\hat{u}_{j}=\left\{\begin{array}{ll}
u_{v, j}^{*} & \text { if } u_{v, j}^{*} \in\left[u_{j}^{-}, u_{j}^{+}\right] \\
u_{j}^{-} & \text {if } u_{v, j}^{*}<u_{j}^{-} \\
u_{j}^{+} & \text {if } u_{v, j}^{*}>u_{j}^{+}
\end{array}, \quad j=1, \ldots, m\right.
$$

to ensure that the input constraints (5.20) are satisfied. Hence, these two different cases are comprised in the case- dependent definition ${ }^{7}$

$$
\widehat{\psi}_{r_{k}+1}^{k}\left(\boldsymbol{\xi}_{r_{1}}^{1}, \ldots, \boldsymbol{\xi}_{r_{m}}^{m}, \boldsymbol{\eta}^{*}, v_{k}\right)=\left\{\begin{array}{lr}
v_{k} & \text { if } u_{v, j}^{*} \in\left[u_{j}^{-}, u_{j}^{+}\right] \\
\frac{\alpha_{k}\left(\boldsymbol{y}_{1}^{*}, \ldots, \boldsymbol{y}_{m}^{*}, \boldsymbol{\eta}^{*}, \hat{\boldsymbol{u}}\right)-\gamma_{r_{k}}^{k}\left(\boldsymbol{\xi}_{r_{k}}^{k}\right)}{\prod_{j=1}^{r_{k}} \frac{\partial \psi_{j}^{k}}{\partial \xi_{j}^{k}}} & \text { else }
\end{array}\right.
$$

for $k=1, \ldots, m$. Note that $\widehat{\psi}_{r_{k}+1}^{k}$ depends on the new input $v_{k}$ as well as on $\boldsymbol{\eta}^{*}$ and all states $\boldsymbol{\xi}_{r_{k}}^{k}, k=1, \ldots, m$ due to the relations (5.35) and (5.37) for the output vectors $\boldsymbol{y}^{*}, k=1, \ldots, m$. By using the new functions (5.53) instead of the previous saturation functions $\psi_{r_{k}+1}^{k}\left(v_{k}, \psi_{r_{k}+1}^{k \pm}\right)$, the BVPs (5.39)-(5.40) can be written as

$$
\begin{aligned}
& \dot{\boldsymbol{\xi}}_{r_{k}}^{k}=\widehat{\boldsymbol{\psi}}_{r_{k}+1}^{k}\left(\boldsymbol{\xi}_{r_{1}}^{1}, \ldots, \boldsymbol{\xi}_{r_{m}}^{m}, \boldsymbol{\eta}^{*}, v_{k}\right), \quad \boldsymbol{\xi}_{r_{k}}^{k}(0)=\boldsymbol{\xi}_{r_{k}, 0}^{k}, \quad \boldsymbol{\xi}_{r_{k}}^{k}(T)=\boldsymbol{\xi}_{r_{k}, T}^{k}, \quad k=1, \ldots, m \\
& \dot{\boldsymbol{\eta}}^{*}=\overline{\boldsymbol{\beta}}\left(\boldsymbol{\eta}^{*}, \boldsymbol{y}_{1}^{*}, y_{1}^{*\left(r_{1}\right)}, \ldots, \boldsymbol{y}_{m}^{*}, y_{m}^{*\left(r_{m}\right)}\right), \quad \boldsymbol{\eta}^{*}(0)=\boldsymbol{\eta}_{0}^{*}, \quad \boldsymbol{\eta}^{*}(T)=\boldsymbol{\eta}_{T}^{*}
\end{aligned}
$$

Both BVPs are fully coupled, since each vector field $\widehat{\boldsymbol{\psi}}_{r_{k}+1}^{k}=\left[\psi_{2}^{k}, \ldots, \psi_{r_{k}}^{k}, \widehat{\psi}_{r_{k}+1}^{k}\right]^{\top}$ depends on all states $\boldsymbol{\xi}_{r_{k}}^{k}, k=1, \ldots, m$ and $\boldsymbol{\eta}^{*}$.

Similar to the SISO case, conflicts may occur between the output constraints $y_{k}^{*(i)} \in$ $\left[y_{k, i}^{-}, y_{k, i}^{+}\right], i=0,1, \ldots, r_{k}-1$ and the input constraints $u_{k}^{*} \in\left[u_{k}^{-}, u_{k}^{+}\right], k=1, \ldots, m$. This can be explained in an illustrative manner for a nonlinear MIMO system with $m=2$ inputs and outputs. Figure 5.2 schematically shows the admissible input set $\mathcal{U}$ and the corresponding set $\mathcal{Y}$ of admissible output derivatives $\left(y_{1}^{*\left(r_{1}\right)}, y_{2}^{\left(r_{2}\right)}\right) \in \mathcal{Y}$ defined by (5.28) and (5.29). If e.g. the first output $y_{1}^{*}=\psi_{1}^{1}\left(\xi_{1}^{1}, \psi_{1}^{1 \pm}\right)$ approaches one of the respective constraints $\psi_{1}^{1 \pm}=y_{1,0}^{ \pm}$ with $\partial \psi_{1}^{1} / \partial \xi_{1}^{1} \rightarrow 0$, then the output derivatives up to $y_{1}^{*\left(r_{1}\right)} \rightarrow 0$ also tend to zero, such that the respective point $\left(y_{1}^{*\left(r_{1}\right)}, y_{2}^{*\left(r_{2}\right)}\right) \in \mathcal{Y}$ in Figure 5.2 will approach the $y_{2}^{*\left(r_{2}\right)}$-axis. However, depending on the input-output dynamics (5.11a)-(5.11b) and the current output variables $\boldsymbol{y}_{k}^{*}, k=1, \ldots, m$ and $\boldsymbol{\eta}^{*}$, the set $\mathcal{Y}$ may move in the direction of the $y_{1}^{*\left(r_{1}\right)}$-axis, such that the $y_{2}^{*\left(r_{2}\right)}$-axis finally leaves the set $\mathcal{Y}$, i.e. $\left(0, y_{2}^{*\left(r_{2}\right)}\right) \notin \mathcal{Y}$. In order to steer $y_{1}^{*\left(r_{1}\right)}$ away from zero to stay inside the set $\mathcal{Y}$, the function $\widehat{\psi}_{r_{1}+1}^{1}$ in (5.49) $(\mathrm{k}=1)$ must assume large values due to the vanishing product $\prod_{j=1}^{r_{1}} \partial \psi_{j}^{1} / \partial \xi_{j}^{1} \rightarrow 0$. This might lead to singularities in the BVPs (5.54)-(5.55) such that their solvability becomes ill-conditioned or impossible (see Section 4.3 and Example 4.2 for details).

\footnotetext{
${ }^{7}$ As explained in Section 4.3 (Footnote 4 ) for the SISO case, the BCs (5.45) still hold for the new inputs $v_{k}$ if continuity of the feedforward controls $u_{k}^{*}(t), k=1, \ldots, m$ at $t=0$ and $t=T$ is desired.
} 
Example 5.5 (Example 5.4 continued). In order to account for input constraints $u_{k}^{*} \in\left[u_{k}^{-}, u_{k}^{+}\right]$ in addition to the output constraints $y_{k}^{*} \in\left[y_{k, 0}^{-}, y_{k, 0}^{+}\right], k=1,2$, the new functions (5.53) with (5.50)-(5.52) replace the saturation functions $\psi_{2}^{k}\left(v_{k}, \psi_{2}^{k \pm}\left(\xi^{k}\right)\right)$ in the BVPs (5.47):

$$
\begin{aligned}
& \dot{\xi}^{1}=\widehat{\psi}_{2}^{1}\left(\xi^{1}, \xi^{2}, \eta^{*}, v_{1}\right), \quad \xi^{1}(0)=\xi_{0}^{1}, \quad \xi^{1}(T)=\xi_{T}^{1}, \\
& \dot{\xi}^{2}=\widehat{\psi}_{2}^{2}\left(\xi^{1}, \xi^{2}, \eta^{*}, v_{2}\right), \quad \xi^{2}(0)=\xi_{0}^{2}, \quad \xi^{2}(T)=\xi_{T}^{2}, \\
& \dot{\eta}^{*}=-6 \eta^{*}+\dot{y}_{1}^{*}+9 y_{2}^{*}+3 \dot{y}_{2}^{*}, \quad \eta^{*}(0)=\eta_{0}^{*}, \quad \eta^{*}(T)=\eta_{T}^{*} \text {. } \\
& =\overline{\boldsymbol{\beta}}\left(\eta^{*}, y_{1}^{*}, \dot{y}_{1}^{*}, y_{2}^{*}, \dot{y}_{2}^{*}\right) \text {. }
\end{aligned}
$$

and in the output equations (5.48):

$$
y_{k}^{*}=\psi_{1}^{k}\left(\xi^{k}, \psi_{1}^{k \pm}\right), \quad \dot{y}_{k}^{*}=\frac{\partial \psi_{1}^{k}}{\partial \xi^{k}} \widehat{\psi}_{2}^{k}\left(\xi^{1}, \xi^{2}, \eta^{*}, v_{k}\right), \quad k=1,2 .
$$

Thereby, the input-output dynamics and the feedforward control of the linear MIMO example follow from (5.11a)-(5.11b) and (5.18), respectively. The saturation functions $\psi_{1}^{k}\left(v_{k}, \psi_{1}^{k \pm}\right)$, the new inputs $v_{k}$, and the boundary values $\xi_{0}^{k}$ and $\xi_{T}^{k}, k=1,2$ are taken from Example 5.4.

Figure 5.6 shows the numerical results for the same scenario as in Figure 5.5 with the same initial guess for bvp4c and different combinations of input/output constraints. If the inputs $u_{1}^{*}(t)$
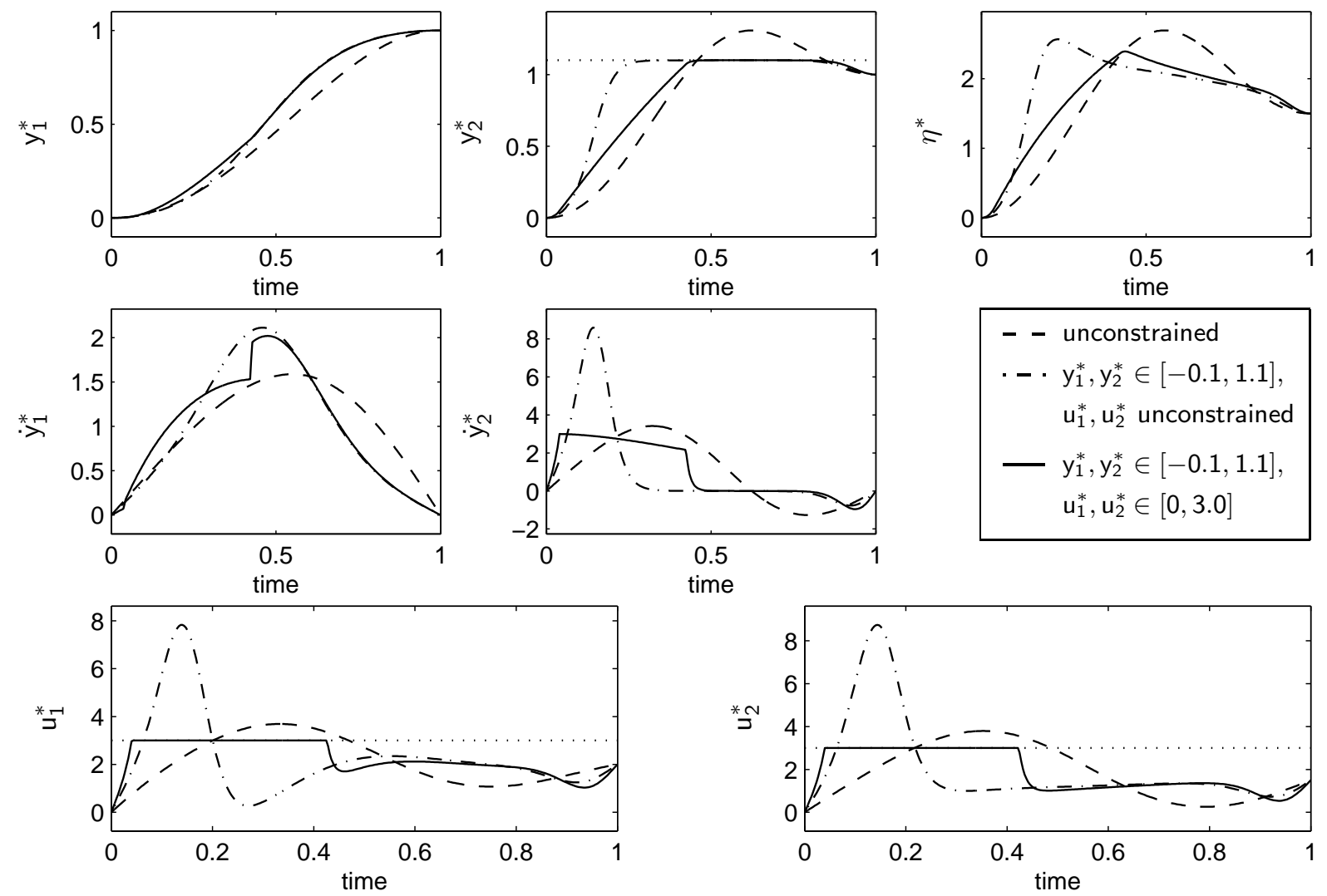

Figure 5.6: Setpoint transition $y_{1,0}^{*}=y_{2,0}^{*}=0 \rightarrow y_{1, T}^{*}=y_{2, T}^{*}=1$ of the linear MIMO system (5.10) based on the solution of the BVPs (5.56) with different input and output constraints. 
and $u_{2}^{*}(t)$ are unconstrained by setting the limits $u_{1}^{ \pm}$and $u_{2}^{ \pm}$to sufficiently large values, the output $y_{2}^{*}(t)$ stays at the limit $y_{2,0}^{+}=1.1$ for a large time interval, see trajectories (- - .). Moreover, the trajectories (-) show that the large overshoot of $u_{1}^{*}(t)$ and $u_{2}^{*}(t)$ can be significantly reduced by additionally limiting the feedforward controls to $u_{1}^{+}=u_{2}^{+}=3$.

\subsection{Example - Flight maneuver of the 3DOF helicopter}

The 3DOF helicopter laboratory experiment (Quanser Inc.) is a typical example of an underactuated mechanical system. As shown in Figure 5.7, the helicopter basically consists of three hinge-mounted rigid body systems: the base turns around the travel axis with angle $\phi_{1}$ and carries the helicopter arm which rotates around the elevation axis with angle $\phi_{2}$. One end of the arm is attached to a counterweight that tares the weight of the helicopter body as the third mechanical subsystem. The rotation of this body is described by the pitch angle $\phi_{3}$. Two propellers are installed at the ends of the helicopter body. The thrusts $F_{1}$ and $F_{2}$ of the propellers serve as control inputs to the system. Hence, the helicopter is underactuated by one control with respect to the three degrees-of-freedom $\phi_{1}, \phi_{2}$, and $\phi_{3}$. Moreover, the helicopter is subject to constraints on the control inputs $F_{1}$ and $F_{2}$ and on the pitch angle $\phi_{3}$.

In (Kiefer et al., 2004) and (Kugi and Kiefer, 2005), a simplified model covering the essential nonlinearities of the helicopter system is derived in such a way that it is differentially flat (Fliess et al., 1995), (Rathinam and Murray, 1998). A flatness-based controller is used to track the helicopter along desired trajectories for the flat outputs. The flatness-based control concept achieves accurate tracking but does not directly account for the above-mentioned input and pitch angle constraints. With respect to a desired rotation in the travel axis $\phi_{1}$, the flatnessbased control would require a sufficiently slow transition in order to stay inside the constraints.

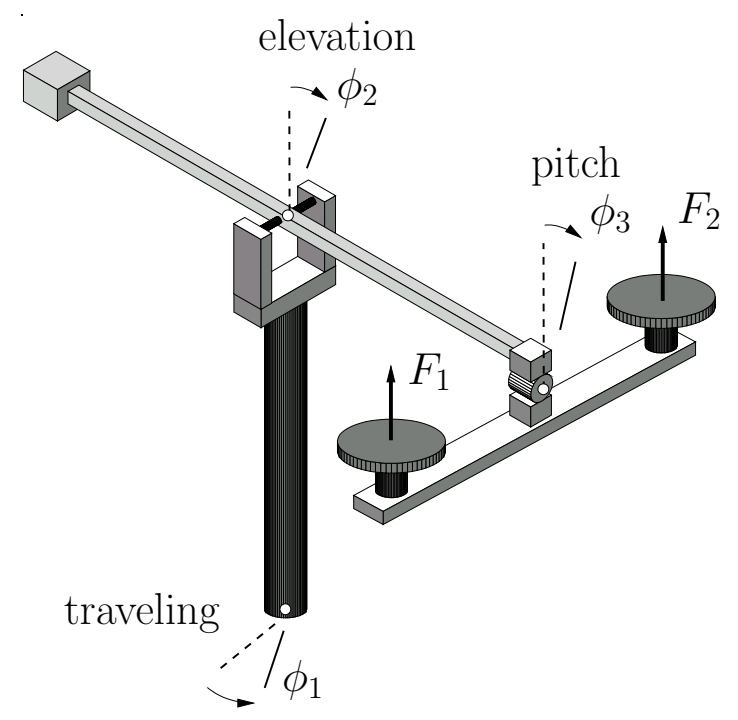

Figure 5.7: Mechanical setup of the 3DOF helicopter. 
In this section, the feedforward control design for nonlinear MIMO systems under input and output constraints is applied to the 3DOF helicopter with two inputs and outputs. The considered flight maneuver is a $360 \mathrm{deg}$-turn around the travel axis. By choosing the pitch angle $\phi_{3}$ as one of the output variable, the constraints on $\phi_{3}$ can be interpreted as output constraints. In order to stabilize the flight maneuver along the nominal trajectories, a time-varying LQ feedback controller is designed. The presented tracking control design for the 3DOF helicopter is a joint work of Kiefer, Kugi, Graichen, and Zeitz (2006) and is verified with the laboratory helicopter at the Chair of System Theory and Automatic Control at Saarland University (Germany).

\subsubsection{Problem statement}

A rigorous mathematical model of the $3 \mathrm{DOF}$ helicopter laboratory experiment as depicted in Figure 5.7 can be derived by utilizing the concept of twists and wrenches (Murray et al., 1994) in combination with the Euler-Lagrange formalism, see (Kiefer et al., 2004) and (Kugi and Kiefer, 2005).

\subsubsection{Helicopter model}

For the feedforward control design, the model of the helicopter in Figure 5.7 can be simplified in such a way that the essential dynamics and nonlinearities are still reproduced. Following the assumptions given in (Kiefer et al., 2004), the equations of motion can be written in the form

$$
\begin{aligned}
& \ddot{\phi}_{1}=b_{1} \sin \left(\phi_{3}\right) \cos \left(\phi_{2}\right) u_{1} \\
& \ddot{\phi}_{2}=a_{1} \sin \left(\phi_{2}\right)+a_{2} \cos \left(\phi_{2}\right)+b_{2} \cos \left(\phi_{3}\right) u_{1} \\
& \ddot{\phi}_{3}=a_{3} \cos \left(\phi_{2}\right) \sin \left(\phi_{3}\right)+b_{3} u_{2}
\end{aligned}
$$

with the transformed inputs $u_{1}=F_{1}+F_{2}$ and $u_{2}=F_{1}-F_{2}$. Thereby, the friction in each axis is neglected and the mass- and geometry-dependent parameters $a_{i}$ and $b_{i}, i=1,2,3$ are given in Table 5.1.

\begin{tabular}{ccc|ccc}
\hline Symbol & Unit & Value & Symbol & Unit & Value \\
\hline$a_{1}$ & $\frac{\mathrm{rad}}{\mathrm{s}^{2}}$ & 0.3496 & $b_{1}$ & $\frac{\mathrm{rad}}{\mathrm{kg} \mathrm{m}}$ & -0.6333 \\
$a_{2}$ & $\frac{\mathrm{rad}}{\mathrm{s}^{2}}$ & -1.1586 & $b_{2}$ & $\frac{\mathrm{rad}}{\mathrm{kg} \mathrm{m}}$ & -0.6501 \\
$a_{3}$ & $\frac{\mathrm{rad}}{\mathrm{s}^{2}}$ & -0.9035 & $b_{3}$ & $\frac{\mathrm{rad}}{\mathrm{kg} \mathrm{m}}$ & 4.6244 \\
\hline
\end{tabular}

Table 5.1: Parameters of the 3DOF helicopter model (5.58) taken from (Kiefer et al., 2006). 


\subsubsection{Finite-time transition problem}

The transition between two stationary setpoints $\left(\phi_{1,0}^{*}, \phi_{2,0}^{*}, \phi_{3,0}^{*}\right) \rightarrow\left(\phi_{1, T}^{*}, \phi_{2, T}^{*}, \phi_{3, T}^{*}\right)$ is considered as an arbitrary flight maneuver of the $3 \mathrm{DOF}$ helicopter within the finite-time interval $t \in[0, T]$. The trajectory of this transition requires to satisfy the following BCs:

$$
\phi_{k}(0)=\phi_{k, 0}^{*}, \quad \phi_{k}(T)=\phi_{k, T}^{*},\left.\quad \dot{\phi}_{k}\right|_{t=0, T}=0, \quad k=1,2,3 .
$$

The considered flight maneuver of the 3DOF helicopter is a 360 deg-turn around the travel axis, whereby it is desired that the helicopter starts and stops with the elevation angle $\phi_{2}=0$. The boundary values for the pitch angle $\phi_{3}$ are set to zero corresponding to a stationary horizontal position of the rotor arm at the beginning and end of the flight maneuver. This leads to the following boundary values:

$$
\phi_{1,0}^{*}=0, \quad \phi_{1, T}^{*}=2 \pi, \quad \phi_{2,0}^{*}=\phi_{2, T}^{*}=0, \quad \phi_{3,0}^{*}=\phi_{3, T}^{*}=0 .
$$

The inputs $u_{1}$ and $u_{2}$ are subject to the constraints

$$
\begin{aligned}
& u_{1} \in\left[u_{1}^{-}, u_{1}^{+}\right]=[0,0.8] \mathrm{N} \\
& u_{2} \in\left[u_{2}^{-}, u_{2}^{+}\right]=[-0.2,0.2] \mathrm{N},
\end{aligned}
$$

which follow from the physical constraints of the actual propeller forces $F_{1}$ and $F_{2}$. Moreover, the pitch angle $\phi_{3}$ is assumed to be constrained by

$$
\phi_{3} \in\left[\phi_{3}^{-}, \phi_{3}^{+}\right]=[-40,40] \mathrm{deg} .
$$

The pitch angle constraints $\phi_{3}^{ \pm}= \pm 40 \mathrm{deg}$ are chosen to demonstrate the feedforward control design and are not physically motivated. In general, $\phi_{3}^{ \pm}$can assume all values between $\pm 10 \mathrm{deg}$ and $\pm 80 \mathrm{deg}$.

From a mathematical point of view, the 12 BCs (5.59) together with the 3 second-order ODEs (5.58) form a two-point BVP for the states $\phi_{1}, \phi_{2}$, and $\phi_{3}$ depending on the inputs $u_{1}$ and $u_{2}$. The feedforward control design has to calculate nominal feedforward trajectories $u_{1}^{*}(t)$, $u_{2}^{*}(t), t \in[0, T]$, which steer the helicopter between the setpoints (5.59) in open-loop mode while accounting for the constraints (5.60) and (5.61).

\subsubsection{Transition problem in input-output coordinates}

The inversion-based feedforward control for the flight maneuver of the helicopter is designed in the input-output coordinates of the considered system (5.58). Since the 3DOF helicopter has three degrees-of-freedom but only two controls $u_{1}$ and $u_{2}$, some freedom exists concerning the choice of two outputs $y_{1}$ and $y_{2}$. An appropriate pair of the input-output coordinates (5.5) is

$$
y_{1}=\phi_{2}, \quad y_{2}=\phi_{3}, \quad \eta=\phi_{1},
$$

whereby the vector relative degree $\{2,2\}$ is well-defined for $y_{2}=\phi_{3}$ with $\left|\phi_{3}\right| \leq \pi / 2$ and $b_{2}, b_{3} \neq 0$, see Table 5.1. Moreover, by choosing the second output as $y_{2}=\phi_{3}$, the constraints (5.61) can be considered as output constraints as described in this chapter. 
Remark 5.5. It is shown in (Kiefer et al., 2004) and (Kugi and Kiefer, 2005) that the helicopter model (5.58) is differentially flat with the flat outputs $z_{1}=\phi_{1}$ and $z_{2}=\phi_{2}$, which can be used for a feedforward control design. However, drawbacks of the flat output set are that the helicopter (5.58) is not statically feedback linearizable and the parameterizations of the states and inputs are highly complex.

The coordinates (5.62) yield the input-output normal form (5.6)-(5.7)

$$
\begin{array}{rlrl}
\ddot{y}_{1} & =a_{1} \sin \left(y_{1}\right)+a_{2} \cos \left(y_{1}\right)+b_{2} \cos \left(y_{2}\right) u_{1} & =\alpha_{1}\left(y_{1}, y_{2}, u_{1}\right) \\
\ddot{y}_{2} & =a_{3} \cos \left(y_{1}\right) \sin \left(y_{2}\right)+b_{3} u_{2} & & =\alpha_{2}\left(y_{1}, y_{2}, u_{2}\right) \\
\ddot{\eta} & =b_{1} \sin \left(y_{2}\right) \cos \left(y_{1}\right) u_{1} & & =\beta\left(y_{1}, y_{2}, u_{1}\right)
\end{array}
$$

with the input-output dynamics (5.63a)-(5.63b) and the internal dynamics (5.63c). The transition between the setpoints (5.59) within the finite time interval $t \in[0, T]$ requires to satisfy the transformed BCs

$$
\begin{aligned}
y_{1}(0) & =\phi_{2,0}^{*}, & y_{1}(T) & =\phi_{2, T}^{*}, & \left.\dot{y}_{1}\right|_{t=0, T} & =0 \\
y_{2}(0) & =\phi_{3,0}^{*}, & y_{2}(T) & =\phi_{3, T}^{*}, & \left.\dot{y}_{2}\right|_{t=0, T} & =0 \\
\eta(0) & =\phi_{1,0}^{*}, & \eta(T) & =\phi_{1, T}^{*}, & \left.\dot{\eta}\right|_{t=0, T} & =0 .
\end{aligned}
$$

The second-order ODEs (5.63), the BCs (5.64), and the constraints (5.60), (5.61) form three nonlinear two-point BVPs for $y_{1}, y_{2}$, and $\eta$ depending on the inputs $u_{1}$ and $u_{2}$.

\subsubsection{Feedforward control design}

The inversion-based design of the feedforward control is based on the inverse input-output dynamics, which can be easily derived in this case, since the input-output dynamics (5.63a)(5.63b) are decoupled with respect to $u_{1}$ and $u_{2}$, i.e.

$$
\begin{aligned}
& u_{1}^{*}=\frac{\ddot{y}_{1}^{*}-a_{1} \sin \left(y_{1}^{*}\right)-a_{2} \cos \left(y_{1}^{*}\right)}{b_{2} \cos \left(y_{2}^{*}\right)}=\alpha_{1}^{-1}\left(y_{1}^{*}, \ddot{y}_{1}^{*}, y_{2}^{*}\right) \\
& u_{2}^{*}=\frac{1}{b_{3}}\left(\ddot{y}_{2}^{*}-a_{3} \cos \left(y_{1}^{*}\right) \sin \left(y_{2}^{*}\right)\right)=\alpha_{2}^{-1}\left(y_{1}^{*}, y_{2}^{*}, \ddot{y}_{2}^{*}\right) .
\end{aligned}
$$

Hence, the feedforward controls $u_{1}^{*}(t)$ and $u_{2}^{*}(t)$ can be algebraically determined for the desired output trajectories $y_{1}^{*}(t)$ and $y_{2}^{*}(t)$, which are assumed as being $\mathcal{C}^{2}$-continuous to ensure continuity of $u_{1}^{*}(t)$ and $u_{2}^{*}(t)$ at the interval bounds $t=0$ and $t=T$.

Obviously, the feedforward controls (5.65) are independent of the internal dynamics state $\eta^{*}$, which represents the traveling of the helicopter, cf. Figure 5.7. However, in order to ensure that the BCs $(5.64 \mathrm{c})$ are satisfied by the trajectory $\eta^{*}(t)$, the BVP of the internal dynamics $(5.63 \mathrm{c})$, $(5.64 \mathrm{c})$ can be rewritten by inserting $(5.65 \mathrm{a})$ into $(5.63 \mathrm{c})$, i.e.

$$
\begin{aligned}
& \ddot{\eta}^{*}=\frac{b_{1}}{b_{2}} \tan \left(y_{2}^{*}\right)\left[\ddot{y}_{1}^{*}-a_{1} \sin \left(y_{1}^{*}\right)-a_{2} \cos \left(y_{1}^{*}\right)\right] \cos \left(y_{1}^{*}\right)=\bar{\beta}\left(y_{1}^{*}, \ddot{y}_{1}^{*}, y_{2}^{*}\right) \\
& \eta^{*}(0)=\phi_{1,0}^{*}, \quad \eta^{*}(T)=\phi_{1, T}^{*},\left.\quad \dot{\eta}\right|_{t=0, T}=0
\end{aligned}
$$


with the trajectories $y_{1}^{*}(t)$ and $y_{2}^{*}(t)$ serving as the input. The BVP (5.66) is overdetermined due to four BCs for one second-order ODE.

Following the approach described in Section 5.1.2, the solvability of the BVP requires 2 free parameters in the setup functions (5.15) for the output trajectories $y_{1}^{*}(t)$ and $y_{2}^{*}(t)$. Thereby, some freedom exists concerning how the free parameters are distributed over the two output functions. The factor $\tan \left(y_{2}^{*}\right)$ in the right-hand side of (5.66) reveals that the internal dynamics is essentially influenced by the second output $y_{2}^{*}$. This is also apparent from a physical point of view, since the acceleration $\ddot{\eta}^{*}=\ddot{\phi}_{1}^{*}$ around the travel axis is directly linked to the pitch angle $y_{2}^{*}=\phi_{3}^{*}$ of the body, see Figure 5.7. Hence, it is reasonable to distribute the parameters (5.16) according to

$$
q_{1}=0, \quad q_{2}=2
$$

such that both parameters are provided in a setup function $\Upsilon_{2}\left(t, \boldsymbol{p}_{2}\right)$ for the second output $y_{2}^{*}(t)=\Upsilon_{2}\left(t, \boldsymbol{p}_{2}\right)$, i.e. $\boldsymbol{p}_{2}=\left(p_{2,1}, p_{2,2}\right)$, whereas the first output is parameterized by a predefined setup function $y_{1}^{*}(t)=\Upsilon_{1}(t)$. In view of the boundary values (5.59) for the $360 \mathrm{deg}$-turn of the helicopter, the first setup function is simply $\Upsilon_{1}(t)=0$, whereas the polynomial (2.31a) (also see Appendix A.1) is used for $\Upsilon_{2}\left(t, \boldsymbol{p}_{2}\right) \in \mathcal{C}^{2}$.

The BVP (5.66) of the internal dynamics with the output trajectories $y_{1}^{*}(t)=\Upsilon_{1}(t)$ and $y_{2}^{*}(t)=$ $\Upsilon_{2}\left(t, \boldsymbol{p}_{2}\right)$ is solved with the MATLAB function bvp4c. The initial guess is a linear interpolation $\eta^{*}\left(t_{k}\right)$ and $\dot{\eta}\left(t_{k}\right)$ between the BCs in (5.66) on a uniform time mesh $t_{k} \in[0, T], k=1, \ldots, 30$ and the parameter guess $\boldsymbol{p}_{2}=\mathbf{0}$. Figure 5.8 shows the nominal trajectories $y_{1}^{*}(t), y_{2}^{*}(t), \eta^{*}(t)$, and the feedforward controls $u_{1}^{*}(t)$ and $u_{2}^{*}(t), t \in[0, T]$ for the 360 deg-turn of the helicopter in the time $T=12 \mathrm{~s}$. The trajectory $\eta^{*}=\phi_{1}^{*}(t)$ is strictly monotonically increasing and constitutes a smooth transition of the helicopter. Figure 5.9 shows time-discrete snapshots of the helicopter
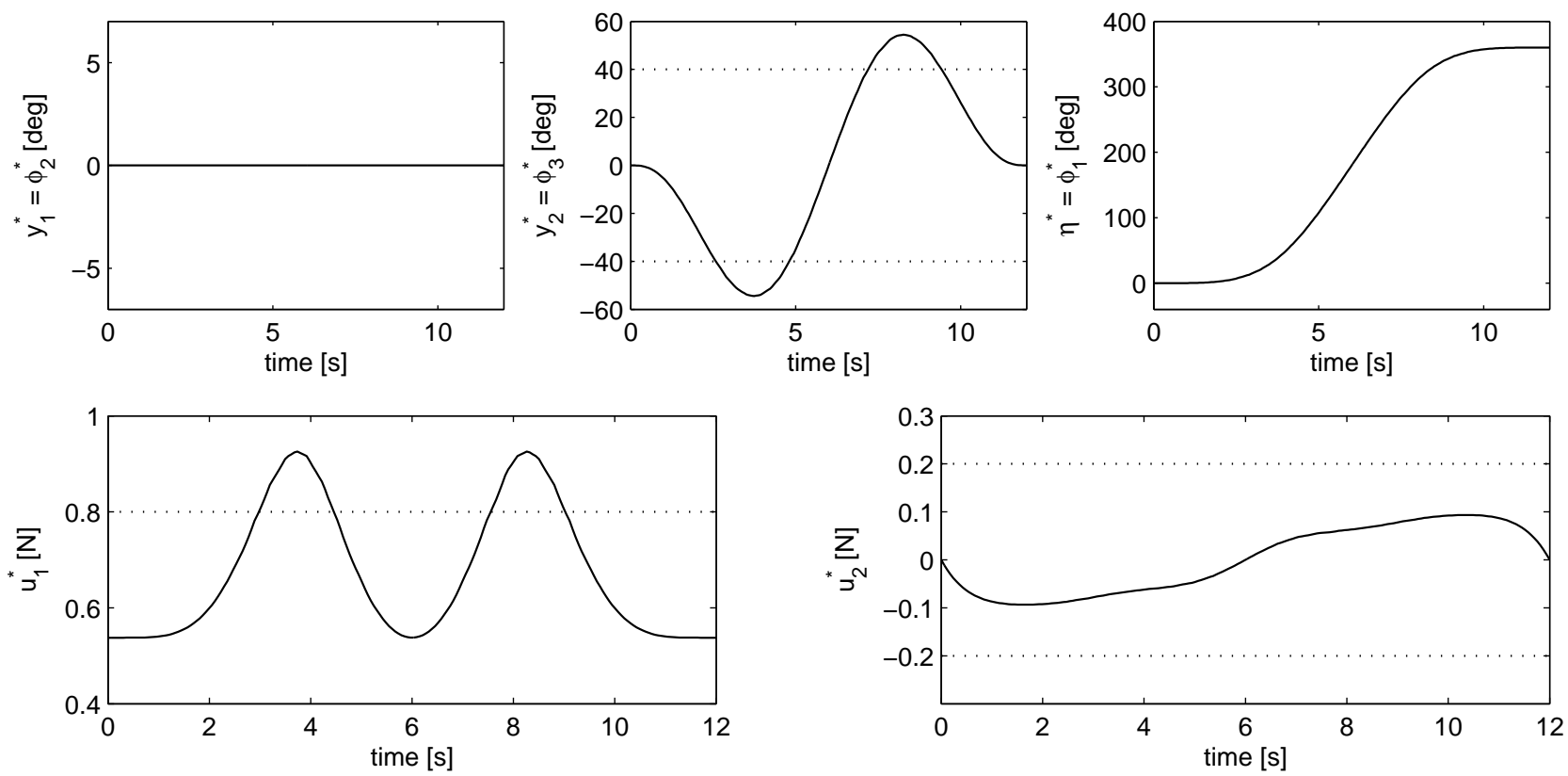

Figure 5.8: Nominal trajectories of the helicopter $360 \mathrm{deg}$-turn with the transition time $T=12 \mathrm{~s}$. 
to illustrate the flight maneuver. The under- and overshoots in the output $y_{2}^{*}(t)=\phi_{3}^{*}(t)$ are required to accelerate and decelerate the helicopter around the travel axis. However, clearly visible in Figure 5.8 is the violation of the constraints (5.60)-(5.61) by $\max _{t} y_{2}^{*}(t)=54.5 \mathrm{deg}$ and $\max _{t} u_{1}^{*}(t)=0.93 \mathrm{~N}$, which are not accounted for by solving the internal dynamics BVP (5.66).

Sequence $1: t \in[0,3] \mathrm{s}$

Sequence $2: t \in[3,4.8] \mathrm{s}$

Sequence $3: t \in[4.8,6] \mathrm{s}$
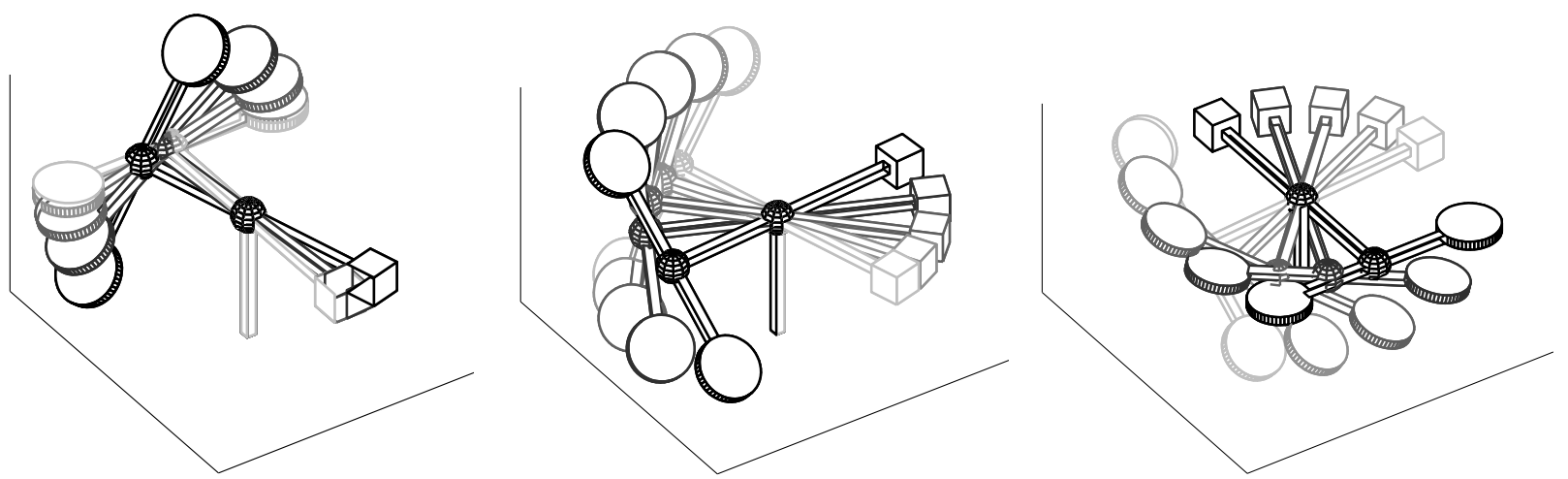

Sequence $4: t \in[6,7.2] \mathrm{s}$

Sequence $5: t \in[7.2,9] \mathrm{s}$

Sequence $6: t \in[9,12] \mathrm{s}$
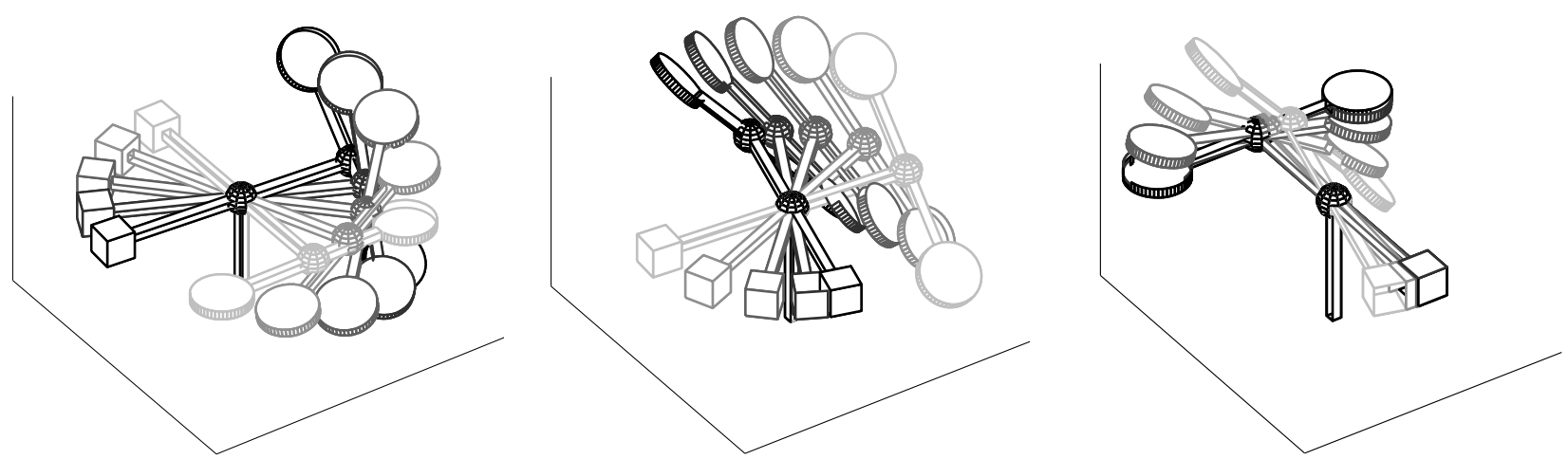

Figure 5.9: Snapshots of the simulated helicopter 360 deg-turn by feedforward control depicted in 6 sequences with increasing darkness of the snapshots as time increases during the respective sequence.

\subsubsection{Incorporation of input constraints}

In a first step, the input constraints (5.60) are systematically considered in the feedforward control design by augmenting the BVP (5.66) of the internal dynamics by the BVPs (5.21) for the outputs $y_{1}^{*}$ and $y_{2}^{*}$ :

$$
\begin{array}{llll}
\ddot{y}_{1}^{*}=\hat{\alpha}_{1}, & y_{1}^{*}(0)=\phi_{2,0}^{*}, & y_{1}^{*}(T)=\phi_{2, T}^{*}, & \left.\dot{y}_{1}^{*}\right|_{t=0, T}=0 \\
\ddot{y}_{2}^{*}=\hat{\alpha}_{2}, & y_{2}^{*}(0)=\phi_{3,0}^{*}, & y_{2}^{*}(T)=\phi_{3, T}^{*}, & \left.\dot{y}_{2}^{*}\right|_{t=0, T}=0 \\
\ddot{\eta}^{*}=\bar{\beta}\left(y_{1}^{*}, \hat{\alpha}_{1}, y_{2}^{*}\right), & \eta^{*}(0)=\phi_{1,0}^{*}, & \eta^{*}(T)=\phi_{1, T}^{*}, & \left.\dot{\eta}^{*}\right|_{t=0, T}=0
\end{array}
$$


The BVPs (5.68) are overdetermined by 12 BCs for 3 second-order ODEs. Hence, 6 free parameters are provided in the sets (5.24)

$$
\boldsymbol{p}_{k}=\left(p_{k, 1}, \ldots, p_{k, q_{k}}\right), \quad k=1,2 \quad \text { with } \quad q_{1}+q_{2}=6
$$

for the setup functions $\Phi_{1}\left(t, \boldsymbol{p}_{1}\right)$ and $\Phi_{2}\left(t, \boldsymbol{p}_{2}\right)$ which are used to parameterize $\hat{\alpha}_{k}=\Phi_{k}\left(t, \boldsymbol{p}_{k}\right)$, $k=1,2$ if the corresponding feedforward controls, cf. (5.25) and (5.65),

$$
u_{\Phi, 1}^{*}=\alpha_{1}^{-1}\left(y_{1}^{*}, \Phi_{1}\left(t, \boldsymbol{p}_{1}\right), y_{2}^{*}\right), \quad u_{\Phi, 2}^{*}=\alpha_{2}^{-1}\left(y_{1}^{*}, y_{2}^{*}, \Phi_{2}\left(t, \boldsymbol{p}_{2}\right)\right)
$$

lie within the constraints (5.60). Thereby, the functions $\Phi_{k}\left(t, \boldsymbol{p}_{k}\right)$ have to satisfy the homogeneous BCs (5.23), i.e. $\Phi_{k}\left(0, \boldsymbol{p}_{k}\right)=\Phi_{k}\left(T, \boldsymbol{p}_{k}\right)=0, k=1,2$. The polynomial (3.6c) is used to parameterize

$$
\Phi_{k}\left(t, \boldsymbol{p}_{k}\right)=\sum_{i=1}^{q_{k}} p_{k, i}\left[\left(\frac{t}{T}\right)^{i+1}-\frac{t}{T}\right], \quad k=1,2 .
$$

A suitable alternative is the sine series (3.6d). If $u_{\Phi, 1}^{*}$ or $u_{\Phi, 2}^{*}$ violate the constraints $u_{k}^{*} \in$ $\left[u_{k}^{-}, u_{k}^{+}\right], k=1,2$, the functions $\hat{\alpha}_{1}$ and $\hat{\alpha}_{2}$ are replanned by the input-output dynamics (5.63a)(5.63b) according to (5.26):

$$
\hat{\alpha}_{1}=\left\{\begin{array}{lll}
\Phi_{1}\left(t, \boldsymbol{p}_{1}\right) & \text { if } u_{\Phi, 1}^{*} \in\left[u_{1}^{-}, u_{1}^{+}\right] \\
\alpha_{1}\left(y_{1}^{*}, y_{2}^{*}, u_{1}^{-}\right) & \text {if } u_{\Phi, 1}^{*}<u_{1}^{-} \\
\alpha_{1}\left(y_{1}^{*}, y_{2}^{*}, u_{1}^{+}\right) & \text {if } u_{\Phi, 1}^{*}>u_{1}^{+}
\end{array} \quad \hat{\alpha}_{2}= \begin{cases}\Phi_{2}\left(t, \boldsymbol{p}_{2}\right) & \text { if } u_{\Phi, 2}^{*} \in\left[u_{2}^{-}, u_{2}^{+}\right] \\
\alpha_{2}\left(y_{1}^{*}, y_{2}^{*}, u_{2}^{-}\right) & \text {if } u_{\Phi, 2}^{*}<u_{2}^{-} \\
\alpha_{2}\left(y_{1}^{*}, y_{2}^{*}, u_{2}^{+}\right) & \text {if } u_{\Phi, 2}^{*}>u_{2}^{+}\end{cases}\right.
$$

Note that the definition of $\hat{\alpha}_{1}$ and $\hat{\alpha}_{2}$ is particularly simple compared to the general case (5.26)-(5.27), since the input-output dynamics (5.63a)-(5.63b) are decoupled with respect to the inputs $u_{1}$ and $u_{2}$.

The assignment of the 6 free parameters in the setup functions $\Phi_{1}\left(t, \boldsymbol{p}_{1}\right)$ and $\Phi_{2}\left(t, \boldsymbol{p}_{2}\right)$ has to be chosen with respect to the condition (5.31), i.e. $q_{1}, q_{2} \geq 2$. Following the discussion for the assignment (5.67) in the unconstrained case, the parameters are allocated to $\boldsymbol{p}_{1}$ and $\boldsymbol{p}_{2}$ by

$$
q_{1}=2, \quad q_{2}=4
$$

in order to leave more "freedom" for the planning of the pitch angle $y_{2}^{*}=\phi_{3}^{*}$.

The BVPs (5.68) with (5.70)-(5.72) are solved with the function bvp4c. A linear interpolation between the corresponding BCs on a uniform mesh with 30 grid points $t_{k} \in[0, T], k=1, \ldots, 30$ serves as reasonable guess for the trajectories $y_{1}^{*}\left(t_{k}\right), y_{2}^{*}\left(t_{k}\right)$, and $\eta^{*}\left(t_{k}\right)$. The initial values for the unknown parameters $\boldsymbol{p}_{1}$ and $\boldsymbol{p}_{2}$ are set to zero. Figure 5.10 shows the nominal trajectories $y_{1}^{*}(t)$, $y_{2}^{*}(t), \eta^{*}(t)$, and the constrained feedforward controls $u_{1}^{*}(t), u_{2}^{*}(t)$ for the 360 deg-turn of the helicopter in $T=12 \mathrm{~s}$ compared to the unconstrained trajectories (- - ) taken from Figure 5.8. It is remarkable that the first output $y_{1}^{*}(t)$ is not identically zero as in the unconstrained case. This is due to the fact that in the time intervals when $u_{1}^{*}(t)$ touches the upper limit $u_{1}^{+}=0.8 \mathrm{~N}$, the setup function $\hat{\alpha}_{1}=\ddot{y}_{1}$ in (5.72) is replanned by $\hat{\alpha}_{1}=\alpha_{1}\left(y_{1}^{*}, y_{2}^{*}, u_{1}^{+}\right)$. Moreover, 
the decoupling of the input-output dynamics (5.68a)-(5.68b) with respect to the inputs $u_{1}$ and $u_{2}$ is also apparent in Figure 5.10. The minimal difference between the trajectories $y_{2}^{*}(t)$ and $u_{2}^{*}(t)$ in the input-constrained (-) and unconstrained case $(--)$ is due to the fact that the second input-output dynamics (5.68b) is only affected by $y_{1}^{*}(t)$ and not $u_{1}^{*}(t)$.
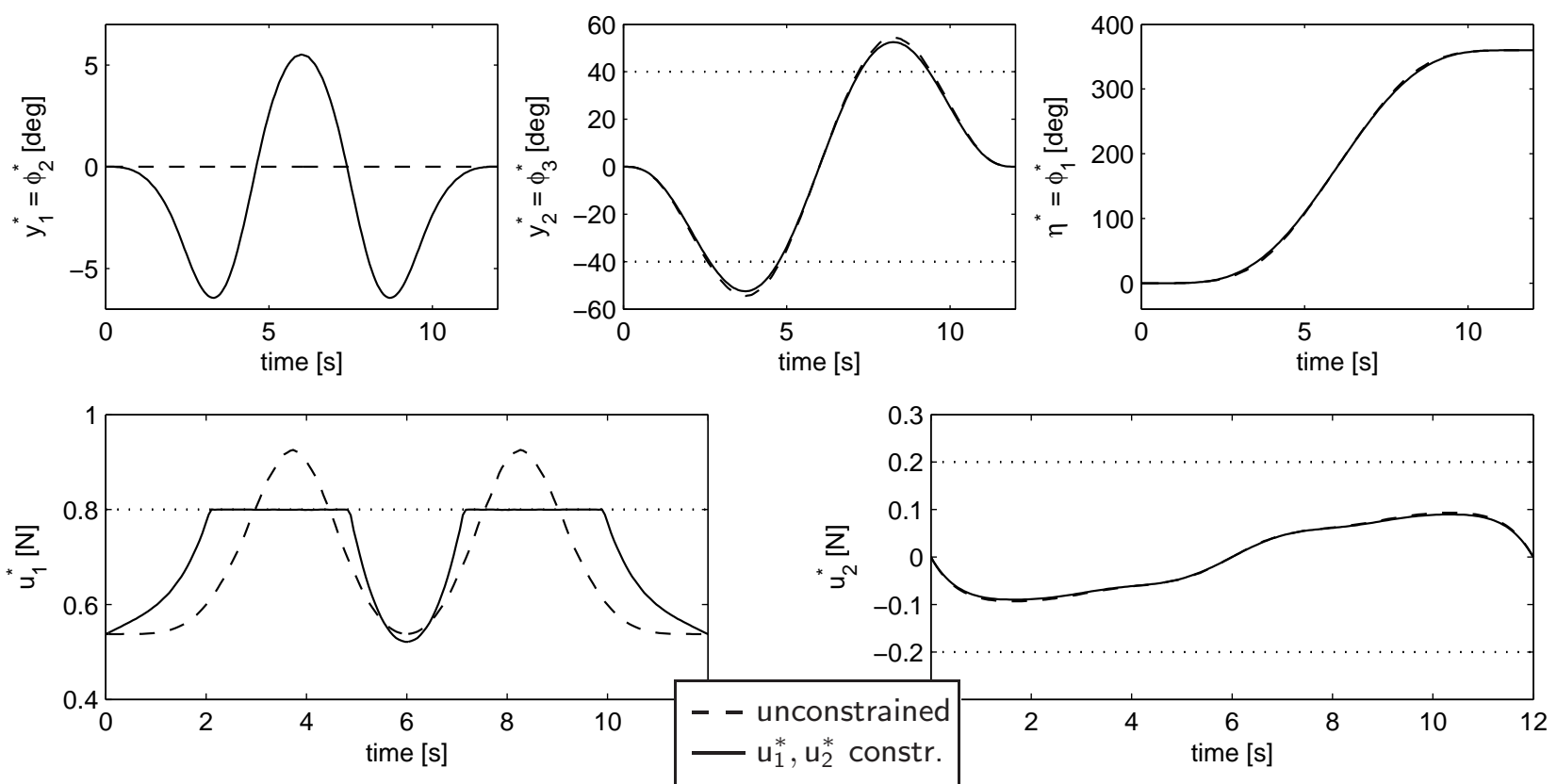

Figure 5.10: Nominal trajectories of the helicopter $360 \mathrm{deg}$-turn in $T=12 \mathrm{~s}$ subject to the input constraints (5.60).

\subsubsection{Incorporation of output constraints}

So far, only the input constraints (5.60) of the helicopter are incorporated in the feedforward control design. In a next step, the constraints (5.61) on the pitch angle are treated as output constraints

$$
y_{2}^{*} \in\left[\phi_{3}^{-}, \phi_{3}^{+}\right]
$$

due to the particular choice of the input-output coordinates (5.62). Hence, a new dynamic system for the output trajectory $y_{2}^{*}(t)$ can be constructed by means of saturation functions as described in Chapter 4. The consideration of constraints for both outputs $y_{1}^{*}(t)$ and $y_{2}^{*}(t)$ (see the MIMO sections 5.3 and 5.49) is not necessary for the helicopter, since only $y_{2}^{*}$ and not $y_{1}^{*}$ is constrained.

\subsubsection{System representation for constrained output}

The output constraint (5.74) is considered by introducing the saturation function (4.3)

$$
y_{2}^{*}=\psi_{1}\left(\xi_{1}, \psi_{1}^{ \pm}\right)
$$


with the new coordinate $\xi_{1} \in \mathbb{R}$ and the asymptotic limits (4.4), i.e. $\psi_{1}^{ \pm}=\phi_{3}^{ \pm}$. The first differentiation of the output $y_{2}^{*}$ gives

$$
\dot{y}_{2}^{*}=\frac{\partial \psi_{1}}{\partial \xi_{1}} \dot{\xi}_{1}
$$

whereby a second coordinate $\xi_{2} \in \mathbb{R}$ is introduced by the ODE

$$
\dot{\xi}_{1}=\psi_{2}\left(\xi_{2}, \psi_{2}^{ \pm}\right)=\xi_{2}
$$

of the first coordinate $\xi_{1}$. Note that in (4.6) the saturation function $\psi_{2}\left(\xi_{2}, \psi_{2}^{ \pm}\right)$is introduced in order to account for constraints on the first output derivative. In this case however, no constraints are defined for $\dot{y}_{2}^{*}$, such that $\psi_{2}\left(\xi_{2}, \psi_{2}^{ \pm}\right)$is replaced by the new coordinate $\xi_{2} \in \mathbb{R}$ (also see Remark 4.1).

A further differentiation of (5.76) yields the second output derivative

$$
\ddot{y}_{2}^{*}=\frac{\partial^{2} \psi_{1}}{\partial \xi_{1}^{2}} \xi_{2}^{2}+\frac{\partial \psi_{1}}{\partial \xi_{1}} \dot{\xi}_{2}
$$

which can be used to incorporate the input constraints $u_{2}^{*} \in\left[u_{2}^{-}, u_{2}^{+}\right]$by means of the function $\hat{\alpha}_{2}=\ddot{y}_{2}^{*}$ in (5.72). Hence, as described in Section 4.3, a new function $\widehat{\psi}_{3}$ with the new input $v$ is introduced for the ODE

$$
\dot{\xi}_{2}=\widehat{\psi}_{3}\left(y_{1}^{*}, \xi_{1}, \xi_{2}, v\right)
$$

according to the definition (4.33):

$$
\widehat{\psi}_{3}\left(y_{1}^{*}, \xi_{1}, \xi_{2}, v\right)= \begin{cases}v & \text { if } u_{2, v}^{*} \in\left[u_{2}^{-}, u_{2}^{+}\right] \\ \frac{\alpha_{2}\left(y_{1}^{*}, y_{2}^{*}, u_{2}^{ \pm}\right)-\frac{\partial^{2} \psi_{1}}{\partial \xi_{1}^{2}} \xi_{2}^{2}}{\frac{\partial \psi_{1}}{\partial \xi_{1}}} \text { if } u_{2, v}^{*} \gtrless u_{2}^{ \pm} .\end{cases}
$$

Note that due to the input-output dynamics (5.63b), the function $\widehat{\psi}_{3}$ depends on the outputs $y_{1}^{*}$ and $y_{2}^{*}$, whereby $y_{2}^{*}$ is given by $(5.75)$ in terms of the new coordinate $\xi_{1}$. In view of (4.32) and $(5.65 \mathrm{~b})$, the input $u_{2, v}^{*}$ denotes the feedforward control which results if the new input $v$ is used to parameterize $\widehat{\psi}_{3}=v$ :

$$
u_{2, v}^{*}=\alpha_{2}^{-1}\left(y_{1}^{*}, y_{2}^{*}, \ddot{y}_{2, v}^{*}\right) \quad \text { with } \quad \ddot{y}_{2, v}^{*}=\frac{\partial^{2} \psi_{1}}{\partial \xi_{1}^{2}} \xi_{2}^{2}+\frac{\partial \psi_{1}}{\partial \xi_{1}} v .
$$

The ODEs (5.77) and (5.79) form a dynamic system with the states $\xi_{1}, \xi_{2}$ and the new input $v$. The output trajectory $y_{2}^{*}(t)$ satisfying the constraints (5.74) can be retraced from (5.75), (5.76), and (5.78).

Remark 5.6. The function $\widehat{\psi}_{3}$ in $(5.80)$ projects the input constraints $u_{2}^{*} \in\left[u_{2}^{-}, u_{2}^{+}\right]$to constraints on the second output derivative $\ddot{y}_{2}^{*} \in\left[\alpha_{2}^{-}, \alpha_{2}^{+}\right]$by means of the input-output dynamics $(5.63 \mathrm{~b})$ :

$$
\alpha_{2}^{ \pm}=a_{3} \cos \left(y_{1}^{*}\right) \sin \left(y_{2}^{*}\right)+b_{3} u_{2}^{ \pm}=\alpha_{2}\left(y_{1}^{*}, y_{2}^{*}, u_{2}^{ \pm}\right) \quad\left(\text { with } b_{3}>0\right)
$$


As emphasized in Section 4.3, care has to be taken that no conflicts arise between the constraints (5.74) and (5.82). If the output $y_{2}^{*}$ approaches the constraints $y_{2}^{*} \rightarrow \phi_{3}^{-}$or $y_{2}^{*} \rightarrow \phi_{3}^{+}$, the time derivatives (5.76) and (5.78) will approach zero, i.e. $\dot{y}_{2}^{*} \rightarrow 0$ and $\ddot{y}_{2}^{*} \rightarrow 0$. Hence, it must be guaranteed that the projected constraints (5.82) for $\ddot{y}_{2}^{*}$ satisfy the inequality

$$
a_{3} \cos \left(y_{1}^{*}\right) \sin \left(y_{2}^{*}\right)+b_{3} u_{2}^{-}<0<a_{3} \cos \left(y_{1}^{*}\right) \sin \left(y_{2}^{*}\right)+b_{3} u_{2}^{+}
$$

if $y_{2}^{*} \rightarrow \phi_{3}^{-}$or $y_{2}^{*} \rightarrow \phi_{3}^{+}$holds. The condition (5.83) can be checked by estimating conservative bounds for the input constraints $u_{2}^{-}$and $u_{2}^{+}$. With the parameters $a_{3}<0$ and $b_{3}>0$ (see Table 5.1), Inequality (5.83) can be written as

$$
b_{3} u_{2}^{-}<\underbrace{-a_{3} \cos \left(y_{1}^{*}\right) \sin \left(y_{2}^{*}\right)}_{-\left|a_{3}\right| \leq 0 \leq\left|a_{3}\right|}<b_{3} u_{2}^{+} .
$$

Hence, if the input constraints $u_{2}^{ \pm}$satisfy

$$
u_{2}^{-}<-\frac{\left|a_{3}\right|}{b_{3}}, \quad u_{2}^{+}>\frac{\left|a_{3}\right|}{b_{3}},
$$

the condition $\ddot{y}_{2}^{-}<0<\ddot{y}_{2}^{+}$is ensured. With the model parameters in Table 5.1, the conservative estimation $u_{2}^{-}<-0.195 \mathrm{~N}$ and $u_{2}^{+}>0.195 \mathrm{~N}$ is satisfied by the actual constraints $u_{2}^{ \pm}= \pm 0.2 \mathrm{~N}$.

\subsubsection{Boundary value problem for constrained output}

To incorporate the output constraint (5.74) in the feedforward control design, the BVP (5.68b) of the output $y_{2}^{*}$ is substituted by a BVP of the new system (5.77), (5.79):

$$
\begin{array}{lll}
\dot{\xi}_{1}=\xi_{2}, & \xi_{1}(0)=\xi_{1,0}, & \xi_{1}(T)=\xi_{1, T} \\
\dot{\xi}_{2}=\widehat{\psi}_{3}\left(y_{1}^{*}, \xi_{1}, \xi_{2}, v\right), & \xi_{2}(0)=\xi_{2,0}, & \xi_{2}(T)=\xi_{2, T} .
\end{array}
$$

The remaining BVPs (5.68a), (5.68c) and the new BVP (5.86) are algebraically coupled by the new coordinates $\xi_{1}, \xi_{2}$ and the output variables $y_{2}^{*}, \ddot{y}_{2}^{*}$ via the relations (5.75) and (5.78).

The boundary values $\xi_{1, i}$ and $\xi_{2, i}, i=0, T$ of the states $\xi_{1}$ and $\xi_{2}$ follow from the BCs in (5.68b) for the output $y_{2}^{*}$. The BCs for the first state $\xi_{1}$ are determined by $y_{2}^{*}(0)=\phi_{3,0}^{*}=0$ and $y_{2}^{*}(T)=\phi_{3, T}^{*}=0$ by inverting the saturation function (5.75):

$$
\xi_{1, i}=\psi_{1}^{-1}\left(\phi_{3, i}^{*}, \psi_{1}^{ \pm}\right)=0, \quad i=0, T .
$$

Thereby, $\xi_{1,0}$ and $\xi_{1, T}$ are zero due to the assumed symmetric constraints $\phi_{3}^{+}=-\phi_{3}^{-}$in (5.61), which ensure that the saturation function (4.12) passes through the origin (also see Figure 4.1). Inserting the homogeneous BCs $\dot{y}_{2}^{*}(0)=\dot{y}_{2}^{*}(T)=0$ in (5.76) leads to $\dot{\xi}_{1}(0)=\dot{\xi}_{1}(T)=0$ (with $\left.\partial \psi_{1} / \partial \xi_{1}>0\right)$. Hence, in view of (5.77), the BCs for $\xi_{2}$ are also homogeneous with

$$
\xi_{2,0}=\xi_{2, T}=0
$$


The BVPs (5.68a) and (5.68c) for $y_{1}^{*}$ and $\eta^{*}$ together with the new BVP (5.86) for $\xi_{1}(t), \xi_{2}(t)$ are overdetermined by $12 \mathrm{BCs}$ for 6 ODEs. In analogy to the parameter distribution (5.69) in the input-constrained case (Section 5.5.4), the parameter set $\boldsymbol{p}_{2}$ with $q_{2}=4$ free parameters in the second setup function $\Phi_{2}\left(t, \boldsymbol{p}_{2}\right)$ are used to parameterize the new input

$$
v=\Phi_{2}\left(t, p_{2}\right)
$$

in the function $\widehat{\psi}_{3}\left(y_{1}^{*}, \xi_{1}, \xi_{2}, v\right)$.

\subsubsection{Numerical results}

The BVPs (5.68a), (5.68c), and (5.86) are solved with the function bvp4c. As in the previous scenarios, a linear interpolation between the corresponding BCs on a uniform mesh with 30 grid points $t_{k} \in[0, T], k=1, \ldots, 30$ serves as reasonable guess for the trajectories $y_{1}^{*}\left(t_{k}\right)$, $\xi_{1}\left(t_{k}\right), \xi_{2}\left(t_{k}\right)$, and $\eta^{*}\left(t_{k}\right)$. The initial values for the unknown parameters $\boldsymbol{p}_{1}$ and $\boldsymbol{p}_{2}$ are set to zero. Figure 5.11 shows the nominal trajectories (-) for the $360 \mathrm{deg}$-turn of the helicopter compared to the corresponding input-constrained trajectories $(--)$ in Figure 5.10. Thereby, the second output $y_{2}^{*}(t)=\phi_{3}(t)$ (following from (5.75) with $\xi_{1}(t)$ ) stays inside the constraints $\phi_{3}^{ \pm}= \pm 40 \mathrm{deg}$, but an aggressive input $u_{2}^{*}(t)$ is necessary in order to rapidly rotate the rotor arm. In contrast to the input-constrained trajectories $(--)$, the first input $u_{1}^{*}(t)$ now stays within the constraints $u_{1}^{ \pm}$such that the setup function $\hat{\alpha}_{1}$ in (5.72) is given by $\hat{\alpha}_{1}=\Phi_{1}\left(t, \boldsymbol{p}_{1}\right)$ for the whole time interval $t \in[0, T]$. Hence, the output trajectory $y_{1}^{*}(t)$ in $(5.68 \mathrm{a})$ is determined by a double integration of $\ddot{y}_{1}^{*}(t)=\Phi_{1}\left(t, \boldsymbol{p}_{1}\right)$ resulting in a simple linear interpolation between the respective BCs.
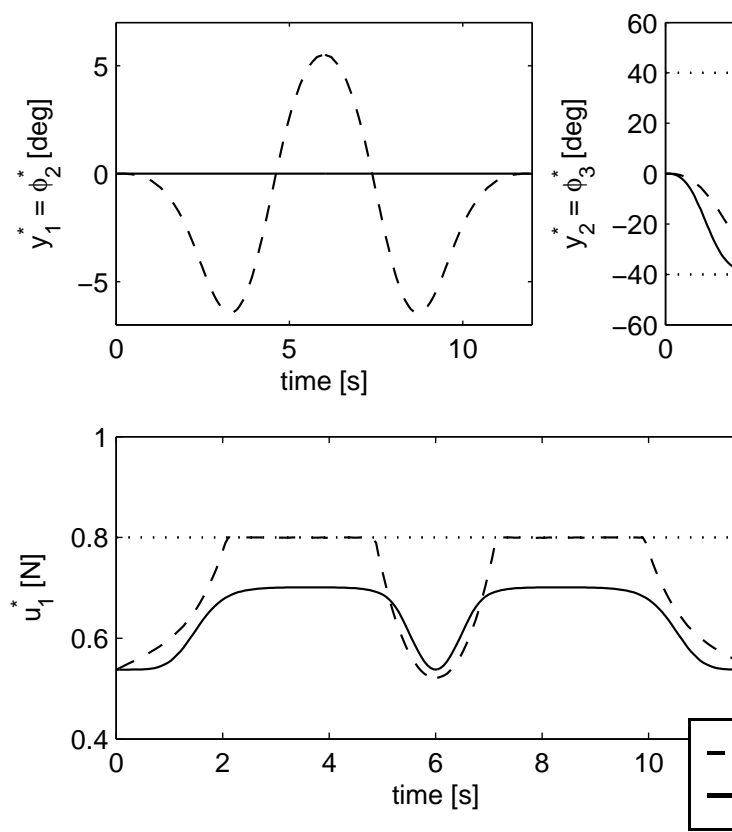
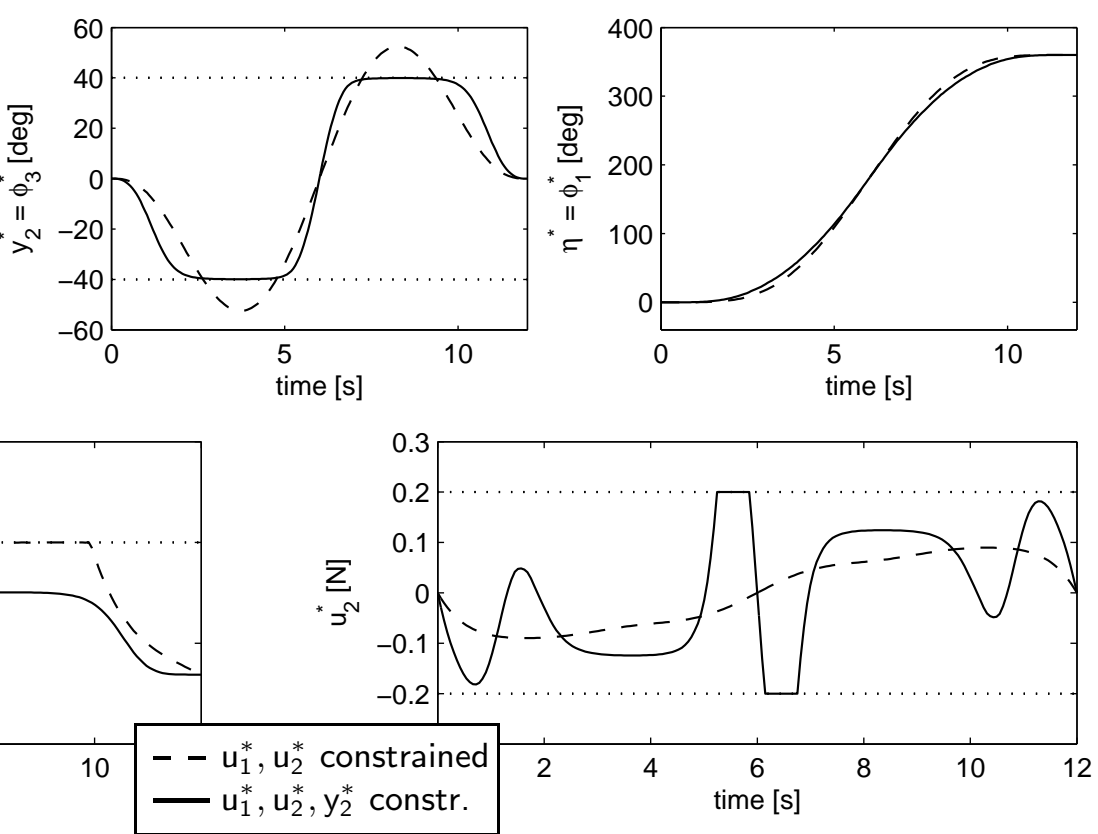

Figure 5.11: Nominal trajectories of the helicopter 360 deg-turn in $T=12 \mathrm{~s}$ subject to the input and output constraints (5.60), (5.74). 


\subsubsection{Feedback controller}

The feedforward trajectories (5.65) provide an open-loop control for the laboratory experiment. Since the model (5.58) results from a simplification of the rigorous model, a closed-loop control is necessary to stabilize the helicopter along the nominal trajectories and to compensate model uncertainties and other disturbances. Similar to the feedback design for the swing-up of the double pendulum (Section 2.4.3.3), the state feedback control for the 3DOF helicopter is based on an optimal LQ design (Kiefer et al., 2006). Thereby, several equations of Section 2.4.3.3 are repeated here to keep the chapter self-contained.

The helicopter model (5.58) can be written in the form $\dot{\boldsymbol{x}}=\boldsymbol{f}(\boldsymbol{x}, \boldsymbol{u})$ with the state $\boldsymbol{x}=$ $\left[\phi_{1}, \dot{\phi}_{1}, \phi_{2}, \dot{\phi}_{2}, \phi_{3}, \dot{\phi}_{3}\right]^{\top}$ and the input vector $\boldsymbol{u}=\left[u_{1}, u_{2}\right]^{\top}$. Linearizing the nonlinear system along the nominal trajectories $\boldsymbol{x}^{*}$ and $\boldsymbol{u}^{*}$ (see trajectories (-) in Figure 5.11) results in the linear time-varying system

$$
\Delta \dot{\boldsymbol{x}}=A(t) \Delta \boldsymbol{x}+B(t) \Delta \boldsymbol{u}
$$

with $A(t)=\left.\frac{\partial \boldsymbol{f}}{\partial \boldsymbol{x}}\right|_{\boldsymbol{x}^{*}(t), \boldsymbol{u}^{*}(t)}$ and $B(t)=\left.\frac{\partial \boldsymbol{f}}{\partial \boldsymbol{u}}\right|_{\boldsymbol{x}^{*}(t), \boldsymbol{u}^{*}(t)}$.

In extension to the control law (2.54) in the SISO case, the closed-loop control vector of the helicopter reads as

$$
\boldsymbol{u}=\boldsymbol{u}^{*}+K(t)\left(\boldsymbol{x}^{*}-\boldsymbol{x}\right)
$$

with the feedforward control $\boldsymbol{u}^{*}(t)$ and the feedback part $\Delta \boldsymbol{u}=K(t)\left(\boldsymbol{x}^{*}-\boldsymbol{x}\right)$. The calculation of the time-varying gain matrix $K(t) \in \mathbb{R}^{2 \times 6}$ is based on an optimal LQ (linear quadratic) feedback design which minimizes the objective functional

$$
I=\Delta \boldsymbol{x}^{\top}(T) M \Delta \boldsymbol{x}(T)+\int_{0}^{T}\left(\Delta \boldsymbol{x}^{\top} Q \Delta \boldsymbol{x}+\Delta \boldsymbol{u} R \Delta \boldsymbol{u}\right) \mathrm{d} t
$$

with the symmetric positive semidefinit matrices $M \in \mathbb{R}^{6 \times 6}, Q \in \mathbb{R}^{6 \times 6}$ and the positive definit matrix $R \in \mathbb{R}^{2 \times 2}$. The gain matrix $K(t)$ follows from the reverse-time integration of the Riccati ODE, see e.g. (Kwakernaak and Sivan, 1972; Bertsekas, 2000),

$$
\dot{P}=-P A(t)-A^{\top}(t) P+P B(t) R^{-1} B^{\top}(t) P-Q, \quad P(T)=M
$$

with

$$
K(t)=R^{-1} B(t) P(t) .
$$

Figure 5.12 shows the entries of the time-varying feedback matrix $K(t)$. Thereby, the two rows of $K(t) \in \mathbb{R}^{2 \times 6}$ are plotted separately. In the control law (5.91), the first row entries $K_{1 i}, i=$ $1, \ldots, 6$ are used to calculate the feedback $\Delta u_{1}$, whereas the second row $K_{2 i}, i=1, \ldots, 6$ gives the feedback $\Delta u_{2}$.

\subsubsection{Experimental results}

The flight maneuver of the 3DOF helicopter is experimentally validated with a laboratory helicopter (Quanser Inc.) at the Chair of System Theory and Automatic Control at Saarland 

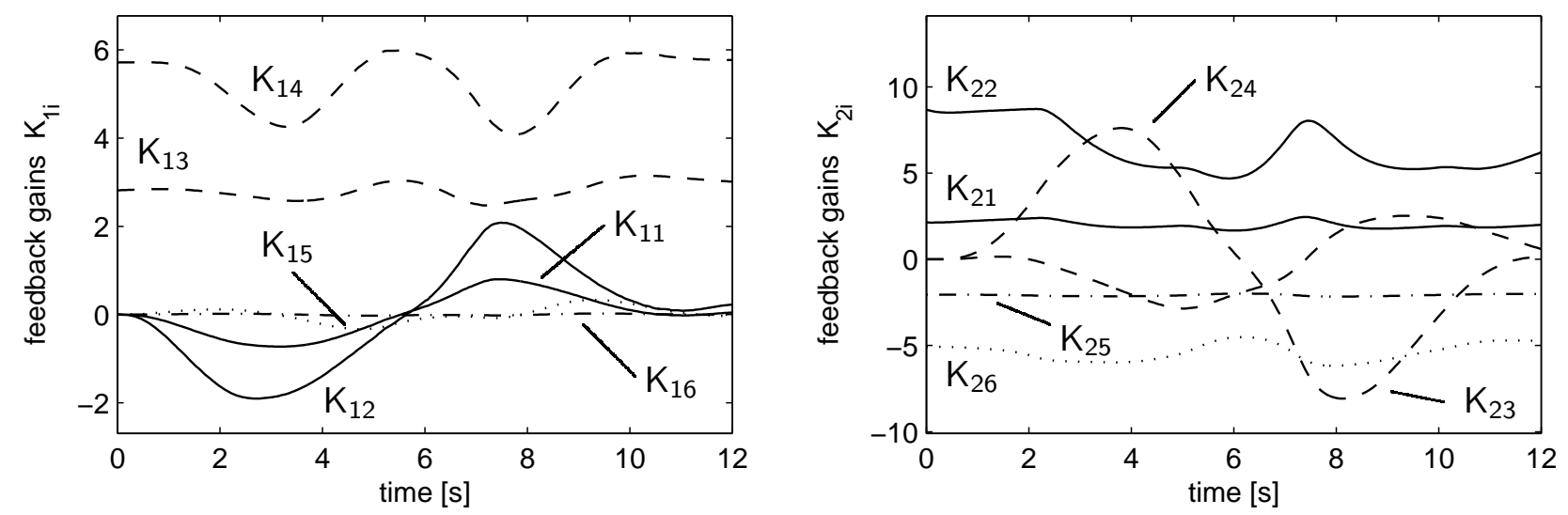

Figure 5.12: Time-varying feedback gains $K_{1 i}(t)$ and $K_{2 i}(t), i=1, \ldots, 6$ of the matrix $K(t) \in \mathbb{R}^{2 \times 6}$ for the $360 \mathrm{deg}$-turn of the 3DOF helicopter (Kiefer et al., 2006).

University (Germany). Figure 5.13 shows the experimental trajectories $\phi_{1}(t), \phi_{2}(t), \phi_{3}(t)$, and the closed-loop inputs $u_{1}(t), u_{2}(t)$ compared to the respective nominal trajectories $(--)$ taken from Figure 5.11. The helicopter starts from the position $\phi_{1,0}^{*}=\phi_{2,0}^{*}=\phi_{3,0}^{*}=0$. The main challenge is the $360 \mathrm{deg}$-rotation of the helicopter base around the travel axis, i.e. $\phi_{1, T}^{*}=2 \pi$ and $\phi_{2, T}^{*}=\phi_{3, T}^{*}=0$. Thereby, the chosen outputs $y_{1}$ and $y_{2}$ show only small deviations from the nominal trajectories $y_{1}^{*}(t)$ and $y_{2}^{*}(t)$. The deviation in the pitch angle $\phi_{3}$ starting at approximately $t=6 \mathrm{~s}$ is caused by the feedback correction $\boldsymbol{u}=K(t)\left(\boldsymbol{x}^{*}-\boldsymbol{x}\right)$ in (5.91), which tries to hold the output $y_{2}=\phi_{1}$ on the desired trajectory $y_{2}^{*}(t)$. This correction is particularly visible in the closed-loop input $u_{2}(t)$ compared to the feedforward trajectory $u_{2}^{*}(t)$. The noise in the inputs $u_{1}(t)$ and $u_{2}(t)$ results from the quantization noise of the incremental encoders where the velocity signals are calculated by approximate differentiation. The comparison of the pitch angle $\eta(t)=\phi_{1}(t)$ with its nominal trajectory $\eta^{*}(t)$ shows that 360 deg-turn of the helicopter is well performed.

\subsection{Conclusions}

The results of the previous chapters are the basis to extend the feedforward control design under input and output constraints to finite-time transition problems of nonlinear MIMO systems. Similar to the SISO case, the feedforward control design treats the setpoint transition as a two-point BVP in the coordinates of the nonlinear MIMO input-output normal form. The constraints on the multiple input and output channels can be incorporated by adapting the results for nonlinear SISO systems. Thereby, the distribution of the free parameters over the multiple outputs leaves some freedom to the user and has to be chosen with respect to the system dynamics and possibly given constraints.

The problem of a not well-defined relative degree (usually requiring a dynamic extension of the system) can be avoided by defining a different set of outputs. Since the shape of the output trajectories is not predefined but is determined by the free parameters in order to solve the 

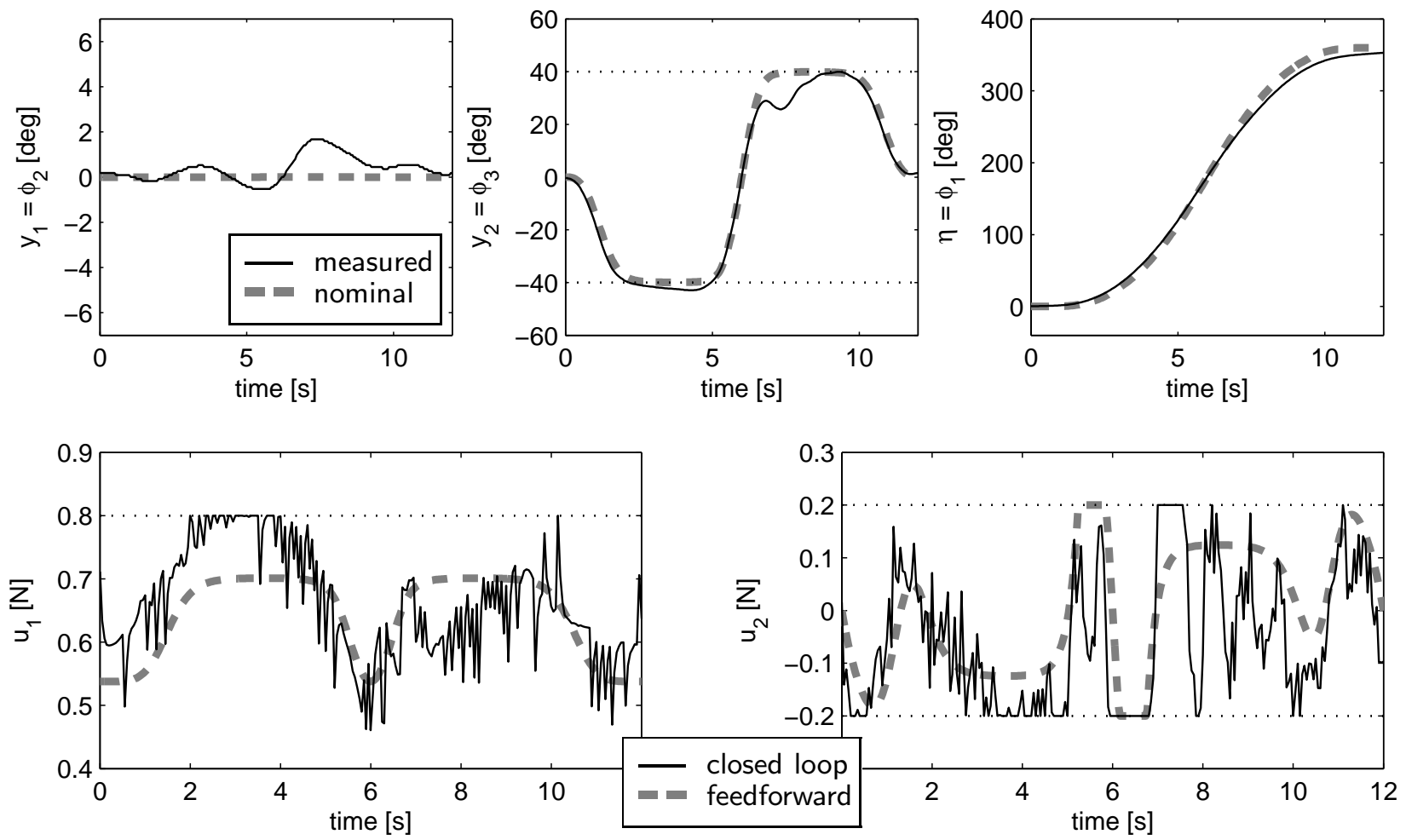

Figure 5.13: Experimental results for the $360 \mathrm{deg}$-turn of the 3DOF helicopter in $T=12 \mathrm{~s}$ (Kiefer et al., 2006).

BVPs, a different choice of outputs can be acceptable to achieve a well--defined relative degree and moreover can significantly reduce the complexity of the analytic expressions.

An illustrative example for the MIMO feedforward control design is the 3DOF helicopter with constraints on both controls and on the pitch angle. The considered flight maneuver is a $360 \mathrm{deg}$-turn around the travel axis. Although the helicopter model is differentially flat, an alternative output set is used in order to interpret the constraints on the pitch angle as output constraints. Moreover, the considered choice of outputs significantly simplifies the feedforward control design compared to the complex parameterizations provided by the flat outputs. 


\section{Chapter 6}

\section{Conclusions and outlook}

The finite-time transition between stationary setpoints is a typical field of application for feedforward control design. In this thesis, the finite-time transition problem is treated as a two-point BVP in the coordinates of the input-output normal form. In the unconstrained case, the transition problem can be reduced to the BVP of the internal dynamics with free parameters provided in the setup of the output trajectory. This concept is illustrated for the swing-up of the double pendulum on a cart.

Constraints on the feedforward control are incorporated with an additional BVP for the output. Moreover, the input constraints provide a means to calculate the transition time as part of the BVP solution with respect to a desired aggressiveness of the feedforward control. The feedforward control design under input constraints is exemplified for a reactor model (CSTR) with constrained cooling power.

In order to account for constraints on the output and its time derivatives, a new dynamic system is constructed which (together with the respective BCs) replaces the previous output BVP. This new system for the constrained output can e.g. be used to minimize the typical counter-swing behavior of nonminimum-phase systems. The side-stepping maneuver of the triple inverted pendulum illustrates this approach by considering constraints on the cart position, velocity, and acceleration.

Moreover, the SISO feedforward control design under input and output constraints can be extended to nonlinear MIMO systems in a straightforward manner with additional degrees-offreedom concerning how the free parameters are distributed to the multiple output trajectories. The MIMO design is applied to a flight maneuver of a 3DOF helicopter with constraints on both inputs and the pitch angle.

The swing-up/side-stepping of the double/triple pendulum and the flight maneuver of the 3DOF helicopter are experimentally validated by supporting the feedforward control with an additional feedback control in the spirit of the two-degree-of-freedom control scheme in Figure 1.1 (also see Figure 2.8). Due to the accuracy of the nonlinear feedforward control, the feedback part is designed by linear methods with the respective system model linearized along 
the nominal trajectories. Thereby, the measurement results of the actuator trajectories reveal that the finite-time transitions are primarily performed by the feedforward control, whereas the feedback part stabilizes the pendulums and the 3DOF helicopter.

The main idea of the presented feedforward control for finite-time transition problems is to systematically incorporate the input and output constraints in the formulation of the BVPs. This is due to the fact that maintaining the BVP representation of the finite-time transition problem has several distinct advantages:

- The stationary BCs ensure that the transition between the initial and terminal setpoint is performed in finite-time. Hence, the input and output trajectories are constant outside the transition interval $t \in[0, T]$ and the typical noncausality of the feedforward control in the case of nonminimum-phase systems is avoided.

- The solution of two-point BVPs with free parameters is a standard problem in numerics. For instance, MatLaB provides the function bvp4c (Shampine et al., 2000), which can be used straightforwardly to solve the $\operatorname{BVP}(\mathrm{s})$ with free parameters.

- Algebraic methods for the solution of two-point BVPs, e.g. finite-difference or collocation methods (bvp4c), are not based on numerical integration like shooting methods and therefore can be applied to nonminimum-phase and unstable systems.

- The BVPs usually exhibit a remarkable numerical robustness since all states are fixed at both sides of the transition interval. This property is preserved by systematically incorporating the input and output constraints in the BVPs. Illustrative examples are the swing-up of the double pendulum and the constrained side-stepping of the triple inverted pendulum in the fast transition time $T=2.4 \mathrm{~s}$. In both cases, a trivial initial guess is sufficient for the solution of the BVPs, although the pendulum models are highly nonlinear as well as unstable and nonminimum-phase in the upward position.

The last point concerning the good numerical robustness due to the double number of BCs for the ODEs is particularly important. This is apparent for the approach made in Section 3.2 to determine the transition time $T$ as part of the BVP solution, which leads to two further ODEs with only three additional BCs. Hence, the numerical robustness of the solution is clearly reduced and a good initial guess of the time scaling factor is required to obtain a solution.

The solution of the BVPs is based on iterative numerical solution schemes, which requires an offline design of the feedforward control. Therefore, the nominal trajectories have to be calculated beforehand and stored in look-up tables. However, this might require a large storage capacity especially if trajectories have to be stored for a large number of setpoint changes. An interesting alternative is to simply save the values of the free parameters of the setup function, which are required for the solvability of the BVPs. Then, the nominal trajectories can be recovered by online-integrating the respective ODEs (e.g. the internal dynamics for the unconstrained feedforward design), if the numerical integration is well-conditioned. 
An important point is that some freedom exists in the feedforward design concerning the choice of output, since only the output BCs are specified. The shape of the output trajectory is determined by the values of the free parameters in order to solve the overdetermined BVPs. Especially in the MIMO case, a different set of outputs might result in a well-defined relative degree such that the inversion-based feedforward design can be applied.

The feedforward design can also be used for differentially flat systems, where the feedforward control is algebraically determined in terms of the flat output and its time derivatives. Nevertheless, the flatness-based feedforward control design relies on a BVP solution if constraints on the inputs and/or the flat outputs have to be considered. On the other hand, it might be advantageous to use an output set that differs from the flat outputs to reduce the complexity of the analytic expressions (see e.g. the 3DOF helicopter).

The feedforward control design under input constraints has also been applied to infinitedimensional diffusion-convection-reaction systems by using summability methods (Meurer et al., 2005) or Galerkin's method to obtain a finite-dimensional model for the feedforward control design (Wieland, 2005; Wieland et al., 2006).

Several promising extensions of the feedforward control design are thinkable. The idea of incorporating the output constraints within a systematically constructed dynamic system leads to the question to which extend this results can be adapted to general state constraints. Moreover, an interesting extension of the feedforward control design is to provide additional free parameters in the BVPs to minimize a cost functional, e.g. to optimize the energy consumption for the finite-time transition. A further perspective concerns the calculation of the transition time as part of the BVP solution in a different way than proposed in Section 3.2 in order to maintain numerical robustness and to extend the concept to MIMO systems. 


\section{Appendix A}

\section{Setup functions for output trajectory with free parameters}

This appendix describes the construction of the setup functions $\Upsilon(t, \boldsymbol{p})$ in Equation (2.31) (Section 2.2.2) for parameterizing the output trajectory $y^{*}(t)=\Upsilon(t, \boldsymbol{p})$. The function $\Upsilon(t, \boldsymbol{p})$ has to connect the stationary output values $y_{0}^{*}$ and $y_{T}^{*}$ with a desired $C^{\rho}$-continuity. This yields the BCs (2.30), which are repeated for the sake of completeness:

$$
\Upsilon(0, \boldsymbol{p})=y_{0}^{*}, \quad \Upsilon(T, \boldsymbol{p})=y_{T}^{*}, \quad \Upsilon^{(i)}(0, \boldsymbol{p})=\Upsilon^{(i)}(T, \boldsymbol{p})=0, \quad i=1, \ldots, \rho .
$$

Furthermore, it is assumed that the function $\Upsilon(t, \boldsymbol{p})$ provides $q$ free parameters in the set $\boldsymbol{p}=\left(p_{1}, \ldots, p_{q}\right) \cdot{ }^{1}$ The following two subsections provide the undetermined coefficients $a_{i}$ of the polynomial (2.31a) and the cosine series (2.31b) for different continuities of $\Upsilon(t, \boldsymbol{p}) \in \mathcal{C}^{\rho}$ with $\rho=0, \ldots, 5$ and the number $q=1, \ldots, 8$ of free parameters $\boldsymbol{p}=\left(p_{1}, \ldots, p_{q}\right)$.

\section{A.1 Polynomial series}

The polynomial (2.31a) is repeated for the sake of completeness:

$$
\Upsilon(t, \boldsymbol{p})=y_{0}^{*}+\sum_{i=\rho+1}^{2 \rho+1} a_{i}\left(\frac{t}{T}\right)^{i}+\sum_{i=1}^{q} p_{i}\left(\frac{t}{T}\right)^{i+2 \rho+1}
$$

Since $\Upsilon(t, \boldsymbol{p})$ already satisfies the BCs (A.1) for $t=0$, the remaining $\rho+1$ coefficients $a_{i}$ are determined by solving the $\rho+1$ equations stemming from the BCs (A.1) for $t=T$. Thereby, the coefficients $a_{i}$ have the structure

$$
\left[\begin{array}{c}
a_{\rho+1} \\
\vdots \\
a_{2 \rho+1}
\end{array}\right]=\boldsymbol{b}\left(y_{T}^{*}-y_{0}^{*}\right)+\boldsymbol{C}\left[\begin{array}{c}
p_{1} \\
\vdots \\
p_{q}
\end{array}\right]
$$

\footnotetext{
${ }^{1}$ Note that $q=n-r$ holds for the internal dynamics BVP (2.32).
} 


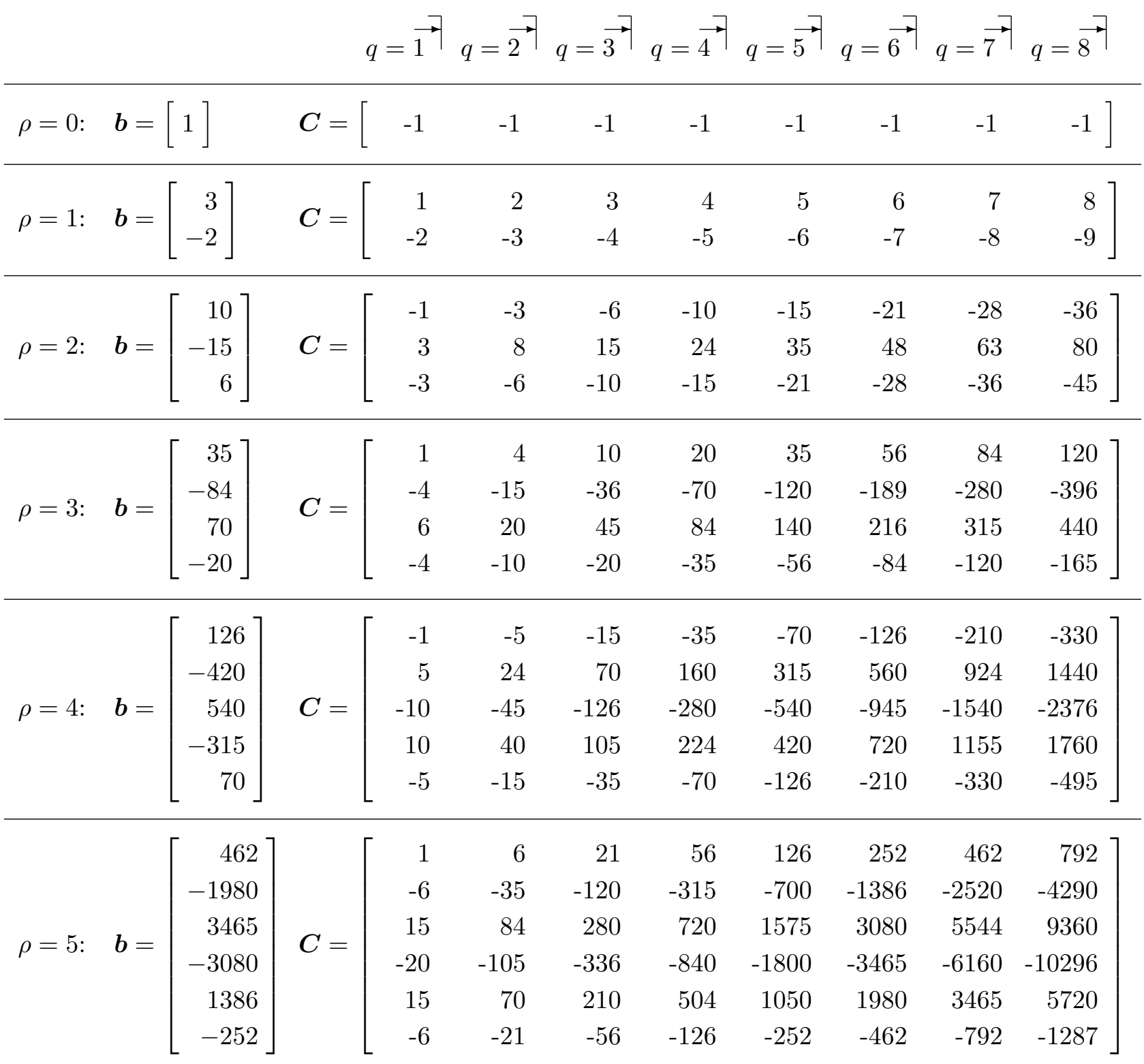

Table A.1: Vector $\boldsymbol{b}$ and matrix $\boldsymbol{C}$ determining the coefficients (A.3) for the polynomial setup (A.2) of $\Upsilon(t, \boldsymbol{p}) \in \mathcal{C}^{\rho}$ depicted for $\rho=0, \ldots, 5$ and various numbers $q=1, \ldots, 8$ of free parameters $\boldsymbol{p}=$ $\left(p_{1}, \ldots, p_{q}\right)$.

with the vector $\boldsymbol{b} \in \mathbb{R}^{\rho+1}$ and the matrix $\boldsymbol{C} \in \mathbb{R}^{\rho+1 \times q}$. $^{2}$ Table A.1 shows the elements of $\boldsymbol{b}$ and $\boldsymbol{C}$ for the polynomial setup of $\Upsilon(t, \boldsymbol{p}) \in \mathcal{C}^{\rho}, \rho=0, \ldots, 5$ and $q=1, \ldots, 8$. Thereby, for the next higher number of free parameters $q$, the matrix $C$ is extended by a further column while the previous columns remain. For instance, for $\rho=1$ and $q=3$ free parameters, the matrix $C$ in Table A.1 reads

$$
\boldsymbol{C}=\left[\begin{array}{rrr}
1 & 2 & 3 \\
-2 & -3 & -4
\end{array}\right]
$$

\footnotetext{
${ }^{2}$ Note that the elements of the vector $\boldsymbol{b} \in \mathbb{R}^{\rho+1}$ can be derived analytically for a monotonically increasing trajectory $\Upsilon(t) \in \mathcal{C}^{\rho}$, see (Piazzi and Visioli, 2001; Graichen et al., 2005a).
} 


\section{A.2 Cosine series}

The cosine series (2.31b) is also repeated for the sake of completeness:

$$
\Upsilon(t, \boldsymbol{p})=\sum_{i=0}^{\bar{\rho}+1} a_{i} \cos \left(\frac{i \pi t}{T}\right)+\sum_{i=1}^{n-r} p_{i} \cos \left(\frac{(\bar{\rho}+1+i) \pi t}{T}\right)
$$

Since the cosine series satisfies the BCs (A.1) for odd time derivatives $i=1,3, \ldots$, the $\bar{\rho}+2$ coefficients $a_{i}$ with

$$
\bar{\rho}= \begin{cases}\rho & \text { if } \rho \text { even } \\ \rho-1 & \text { if } \rho \text { odd }\end{cases}
$$

follow from the set of equations stemming from the $\bar{\rho}+2$ BCs in (A.1) for $\Upsilon^{(i)}(t, \boldsymbol{p})$ with the even numbers $i=0,2, \ldots, \bar{\rho}$. Thereby, the coefficients $a_{i}$ have the same structure as the polynomial coefficients (A.3):

$$
\left[\begin{array}{c}
a_{0} \\
\vdots \\
a_{\bar{\rho}+1}
\end{array}\right]=\boldsymbol{b}\left(y_{T}^{*}-y_{0}^{*}\right)+\boldsymbol{C}\left[\begin{array}{c}
p_{1} \\
\vdots \\
p_{q}
\end{array}\right]
$$

with $\boldsymbol{b} \in \mathbb{R}^{\bar{\rho}+2}$ and $\boldsymbol{C} \in \mathbb{R}^{\bar{\rho}+2 \times q}$. Table A.2 shows the elements of $\boldsymbol{b}$ and $\boldsymbol{C}$ for the setup of $\Upsilon(t, \boldsymbol{p}) \in \mathcal{C}^{\rho}$ by the cosine series (A.4) with $\rho=0, \ldots, 5$ and $q=1, \ldots, 8$. In analogy to Table A.1, the matrix $\boldsymbol{C}$ is extended by further columns for additional free parameters $q$ while the previous columns remain.

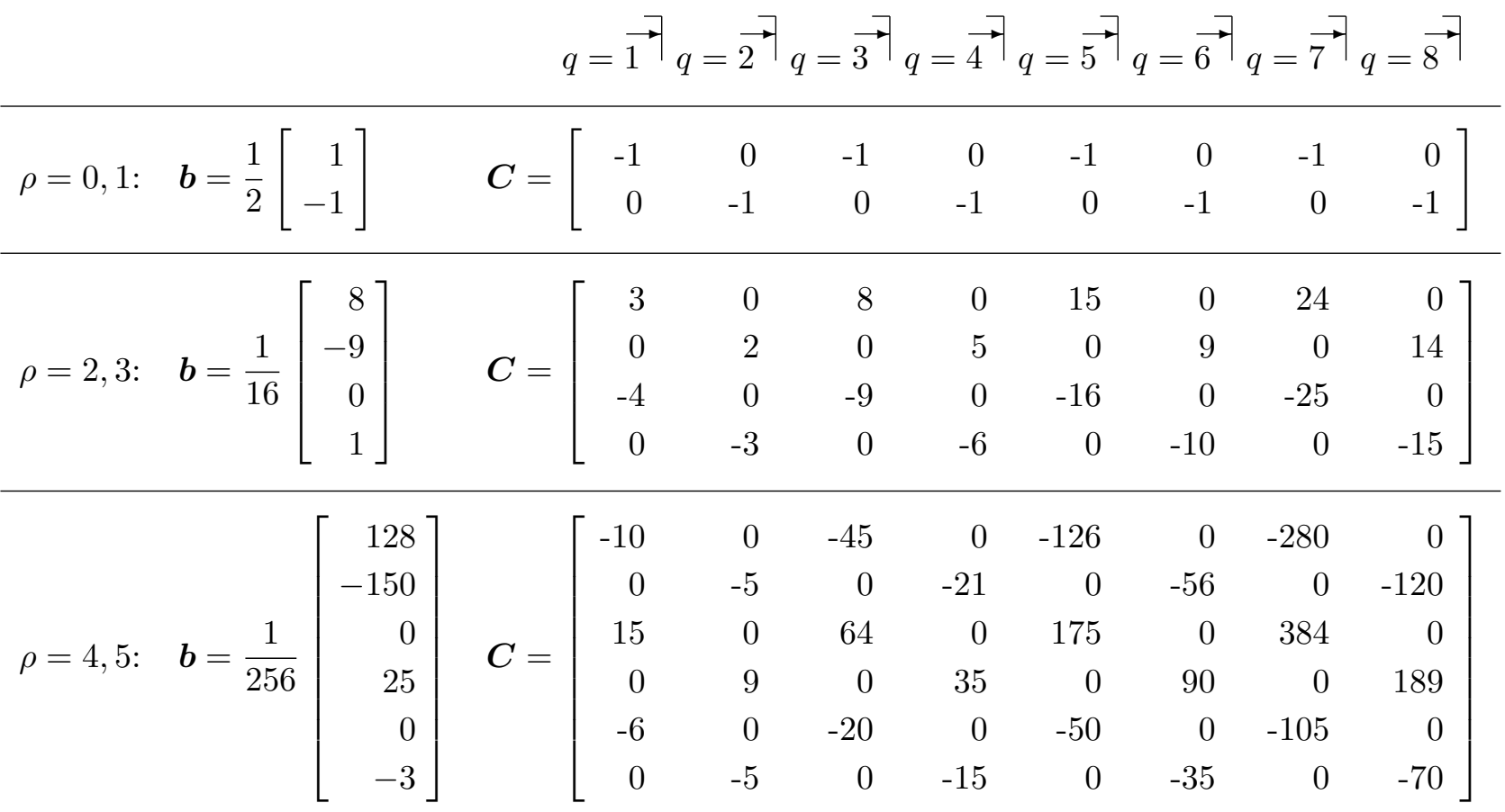

Table A.2: Vector $\boldsymbol{b}$ and matrix $\boldsymbol{C}$ determining the coefficients (A.5) for the cosine series (A.4) as setup of $\Upsilon(t, \boldsymbol{p}) \in \mathcal{C}^{\rho}$ depicted for $\rho=0, \ldots, 5$ and various numbers $q=1, \ldots, 8$ of free parameters $\boldsymbol{p}=\left(p_{1}, \ldots, p_{q}\right)$. 


\section{Appendix B}

\section{Numerical solution of two-point boundary value problems}

Two-point BVPs with free parameters can be solved with a variety of numerical methods. Thereby, the different BVP formulations arising in this thesis can be cast in the general nonlinear form (2.37), which is repeated here for the sake of completeness:

$$
\begin{gathered}
\dot{\boldsymbol{\zeta}}=\boldsymbol{f}(\boldsymbol{\zeta}, t, \boldsymbol{p}), \quad t \in(0, T), \\
\boldsymbol{g}(\boldsymbol{\zeta}(0), \boldsymbol{\zeta}(T), \boldsymbol{p})=\mathbf{0}
\end{gathered}
$$

with the state $\boldsymbol{\zeta} \in \mathbb{R}^{n_{\zeta}}$, the free parameters $\boldsymbol{p} \in \mathbb{R}^{n_{p}}$, and the boundary functions $\boldsymbol{g}$, dim $\boldsymbol{g}=n_{g}$. In view of (2.38), the dimensions of $\boldsymbol{\zeta}, \boldsymbol{p}$, and $\boldsymbol{g}$ have to satisfy the condition

$$
n_{p}=n_{g}-n_{\zeta}
$$

The most common techniques for solving BVPs are collocation, e.g. implemented in the MATLAB function bvp4c (Shampine et al., 2000), finite-difference schemes, and shooting. In this appendix, these methods are shortly introduced in order to illustrate the differences in handling the BVP by either algebraic methods (collocation, finite-differences) or numerical integration (shooting).

\section{B.1 Collocation method}

The basic idea of the collocation method is to divide the time interval $[0, T]$ in subintervals and to approximate the solution $\boldsymbol{\zeta}(t)$ of the BVP (B.1) on each subinterval by appropriate setup functions. Continuity conditions at the subinterval bounds ensure that the overall approximate solution is continuous.

The transition interval $[0, T]$ is divided in $N$ subintervals $\left[t_{i}, t_{i+1}\right], i=0,1, \ldots, N-1$ with the time grid

$$
0=t_{0}<t_{1}<\cdots<t_{N}=T
$$


On each interval $\left[t_{i}, t_{i+1}\right]$, the solution $\boldsymbol{\zeta}(t)$ of the BVP (B.1) is approximated by collocation functions $\hat{\boldsymbol{\zeta}}_{i}(t)$, e.g. by means of polynomials of order $m$ :

$$
\boldsymbol{\zeta}(t) \approx \hat{\boldsymbol{\zeta}}_{i}(t)=\sum_{j=0}^{m} \boldsymbol{a}_{i, j}\left(t-t_{i}\right)^{j}, \quad t \in\left[t_{i}, t_{i+1}\right], \quad i=0,1, \ldots, N-1 .
$$

The polynomial order $m$ can be used to adjust the accuracy of the collocation scheme as explained below. Each function $\hat{\boldsymbol{\zeta}}_{i}(t)$ approximates the solution $\boldsymbol{\zeta}(t) \in \mathbb{R}^{n_{\zeta}}$ on the time interval $t \in\left[t_{i}, t_{i+1}\right]$ in dependence of the $m+1$ coefficient vectors $\boldsymbol{a}_{i, j} \in \mathbb{R}^{n_{\zeta}}$. Hence, the overall number of coefficients to approximate the solution $\boldsymbol{\zeta}(t), t \in[0, T]$ is $(m+1) N n_{\zeta}$.

The functions $\hat{\boldsymbol{\zeta}}_{i}(t)$ have to satisfy the ODEs (B.1a)

$$
\dot{\hat{\boldsymbol{\zeta}}}_{i}\left(t_{i, j}\right)=\boldsymbol{f}\left(\hat{\boldsymbol{\zeta}}_{i}\left(t_{i, j}\right), t_{i, j}, \boldsymbol{p}\right), \quad i=0,1, \ldots, N-1, \quad j=1, \ldots, m
$$

at the collocation points

$$
t_{i, j}=t_{i}+\rho_{j}\left(t_{i+1}-t_{i}\right) \quad \text { with } \quad 0<\rho_{1}<\rho_{2}<\cdots<\rho_{m}<1 .
$$

In order to ensure continuity of the approximate solution over the whole time interval $[0, T]$, the following continuity conditions are imposed on the functions $\hat{\boldsymbol{\zeta}}_{i}(t)$ :

$$
\hat{\boldsymbol{\zeta}}_{i}\left(t_{i}\right)=\hat{\boldsymbol{\zeta}}_{i-1}\left(t_{i}\right), \quad i=1, \ldots, N-1
$$

Finally, the BCs (B.1b) lead to the $n_{g}$ equations

$$
\boldsymbol{g}\left(\hat{\boldsymbol{\zeta}}_{0}(0), \hat{\boldsymbol{\zeta}}_{N-1}(T), \boldsymbol{p}\right)=0
$$

In view of the condition (B.2), the number of equations (B.5a)-(B.5c) exactly matches the number of coefficients $\boldsymbol{a}_{i, j}$ and free parameters $\boldsymbol{p}$ :

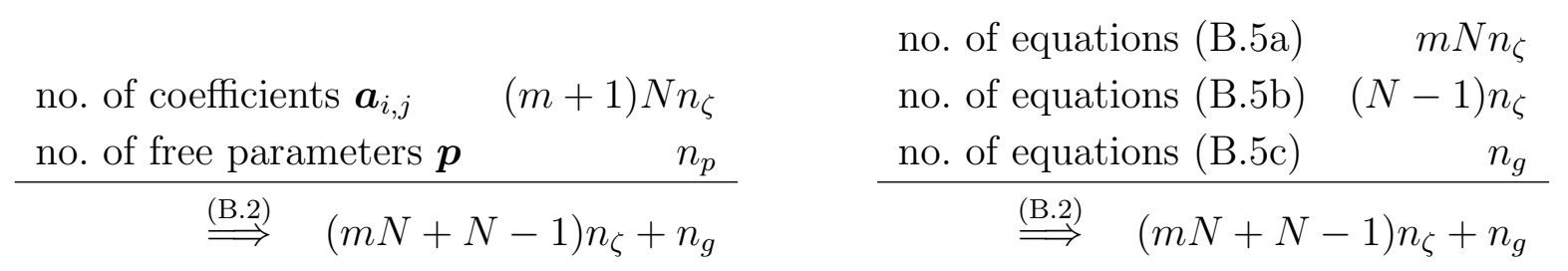

The equations (B.5) form a set of nonlinear algebraic equations for the coefficients $\boldsymbol{a}_{i, j}$ and free parameters $p$, which can be solved iteratively, e.g. by using Newton's method.

According to (Auzinger et al., 2003b, 2002; Ascher et al., 1988), if $\boldsymbol{\zeta}(t)$ is an isolated solution ${ }^{1}$ of the BVP (B.1), then the approximate solution $\hat{\boldsymbol{\zeta}}(t):=\hat{\boldsymbol{\zeta}}_{i}(t), t \in\left[t_{i}, t_{i+1}\right], i=0,1, \ldots, N-1$ of

\footnotetext{
${ }^{1}$ The solution $\boldsymbol{\zeta}(t)$ is said to be isolated, if the variational or linearized problem - i.e. the linearization of the original BVP (B.1) along the solution $\boldsymbol{\zeta}(t)$ - only has the trivial solution. In practice it is usually unknown how many solution exist to a given nonlinear BVP. But the solution of the linearized problem is unique, when the linearization is done at an isolated solution $\boldsymbol{\zeta}(t)$. Iterative methods for solving nonlinear equations usually utilize this fact and proceed locally (Ascher et al., 1988).
} 
the collocation scheme (B.5) can be obtained by Newton's method with quadratic convergence and the following error estimate:

$$
\max _{t \in[0, T]}|\hat{\boldsymbol{\zeta}}(t)-\boldsymbol{\zeta}(t)|=O\left(h^{m+\nu}\right) \quad \text { with } \quad h=\max _{0 \leq i \leq N-1}\left(t_{i+1}-t_{i}\right),
$$

where $m$ is the polynomial order in (B.4) and $\nu=0$ if $m$ is even or $\nu=1$ if $m$ is odd.

The MATLAB function bvp4c is based on the collocation method, whereby $\mathcal{C}^{1}$-continuity of the approximate solution $\hat{\boldsymbol{\zeta}}(t)$ is achieved by further continuity conditions. Another efficient MATLAB solver for BVPs with singularities is sbvp (Auzinger et al., 2003a), which also utilizes the collocation method. ${ }^{2}$

It is remarkable that the collocation method is not restricted to a certain class of ODEs (B.1a). In particular, the collocation method can be used for nonlinear unstable ODEs, since the BVP solution is obtained by algebraically solving the set of equations (B.5) instead of using numerical ODE integration.

\section{B.2 Finite-difference method}

Finite-difference methods are widely used for the numerical solution of partial differential equations in the context of the "method of lines" (Schiesser, 1991; Köhler, 2002; Vande Wouwer et al., 2004). However, finite-difference schemes can also be used for the solution of two-point BVPs as given in (B.1). Similar to the collocation method, the time interval $[0, T]$ is divided in $N$ subintervals with the time grid (B.3). For the sake of simplicity, the time grid is assumed to be uniform with

$$
h=t_{i+1}-t_{i}=\text { const. }, \quad i=0,1, \ldots, N-1,
$$

although non-uniform discretization can be used as well. The difference to the collocation method is that the BVP solution $\boldsymbol{\zeta}(t)$ is not piece-wise approximated by a collocation function, but the first-order derivative $\dot{\boldsymbol{\zeta}}(t)$ in (B.1a) is approximated by finite-differences. Nevertheless, the BVP solution is obtained by solving a set of nonlinear algebraic equations without using numerical ODE integration.

\section{Midpoint scheme}

A common way to approximate the first-order derivative $\dot{\boldsymbol{\zeta}}$ by finite-differences is the midpoint scheme

$$
\dot{\boldsymbol{\zeta}}\left(t_{i+\frac{1}{2}}\right)=\frac{\boldsymbol{\zeta}\left(t_{i+1}\right)-\boldsymbol{\zeta}\left(t_{i}\right)}{h}+O\left(h^{2}\right), \quad i=0,1, \ldots, N-1
$$

with $t_{i+\frac{1}{2}}=\frac{1}{2}\left(t_{i}+t_{i+1}\right)$. The midpoint scheme (B.8) can be used to approximate the solution $\boldsymbol{\zeta}(t)$ at the time points $t_{i}$

$$
\hat{\boldsymbol{\zeta}}_{i}=\boldsymbol{\zeta}\left(t_{i}\right), \quad i=0,1, \ldots, N
$$

\footnotetext{
${ }^{2}$ The documentation and software package for sbvp is available under http://www.math.tuwien.ac.at/ ewa/.
} 
by replacing the ODEs (B.1a) by the $N n_{\zeta}$ algebraic equations

$$
\frac{\hat{\boldsymbol{\zeta}}_{i+1}-\hat{\boldsymbol{\zeta}}_{i}}{h}=\boldsymbol{f}\left(\frac{1}{2}\left(\hat{\boldsymbol{\zeta}}_{i}+\hat{\boldsymbol{\zeta}}_{i+1}\right), t_{i+\frac{1}{2}}, \boldsymbol{p}\right), \quad i=0,1, \ldots, N-1
$$

The BCs (B.1b) lead to additional $n_{g}$ equations

$$
\boldsymbol{g}\left(\hat{\boldsymbol{\zeta}}_{0}, \hat{\boldsymbol{\zeta}}_{N}, \boldsymbol{p}\right)=\mathbf{0}
$$

Hence, in view of the condition (B.2), the number $N n_{\zeta}+n_{g}$ of equations (B.10) equals the number of unknown variables $\hat{\boldsymbol{\zeta}}_{i}$ and $\boldsymbol{p}$. The algebraic equation set (B.10) can be iteratively solved e.g. with Newton's method. Detailed convergence and stability analysis for general one-step schemes can be found e.g. in (Keller, 1968; Ascher et al., 1988).

Remark B.1. An alternative to the midpoint scheme is the trapezoidal scheme, which replaces the equations (B.10a) by

$$
\frac{\hat{\boldsymbol{\zeta}}_{i+1}-\hat{\boldsymbol{\zeta}}_{i}}{h}=\frac{1}{2}\left(\boldsymbol{f}\left(\hat{\boldsymbol{\zeta}}_{i}, t_{i}, \boldsymbol{p}\right)+\boldsymbol{f}\left(\hat{\boldsymbol{\zeta}}_{i+1}, t_{i+1}, \boldsymbol{p}\right)\right), \quad i=0,1, \ldots, N-2 .
$$

The trapezoidal scheme leads to the same approximation error $O\left(h^{2}\right)$, but requires one more evaluation of the function $\boldsymbol{f}$ compared to the midpoint scheme.

\section{Higher-order schemes}

The advantage of the midpoint and trapezoidal schemes is that they are easy to implement, but their accuracy is only of order 2, i.e. the approximation error is $O\left(h^{2}\right)$. One way to increase the accuracy is to use higher-order formulas for one-step schemes. This has the advantage that nonuniform meshes are easily handled and the discretization near the boundaries requires no special treatment (Ascher et al., 1988). However, a typical problem of the higher-order onestep schemes (e.g. of Runge-Kutta type) is that they require several evaluations of the function $\boldsymbol{f}$ in (B.1a) for one time step. An alternative is to use multi-step schemes for higher-order approximations as shortly illustrated in the following.

In general, the $i$-th derivative $\mathrm{d}^{i} \boldsymbol{\zeta} / \mathrm{d} t^{i}$ of a function $\boldsymbol{\zeta}(t)$ can be approximated by the finitedifference scheme

$$
\frac{\mathrm{d}^{i} \boldsymbol{\zeta}}{\mathrm{d} t^{i}}=\frac{i !}{h^{i}} \sum_{j=j_{\min }}^{j_{\max }} c_{j} \boldsymbol{\zeta}(t+j h)+O\left(h^{m}\right)
$$

whereby the approximation error $O\left(h^{m}\right)$ can be freely chosen. The coefficients $c_{j}$ are the solution of the equation set (Fornberg, 1988; Eberly, 2001; Wieland, 2005)

$$
\sum_{j=j_{\min }}^{j_{\max }} j^{k} c_{j}=\left\{\begin{array}{ll}
1 & \text { if } k=i \\
0 & \text { else }
\end{array}, \quad k=0,1, \ldots, i+m-1\right.
$$


Thereby, the index $k$ denotes the single equations of the set. In order to ensure that (B.12) is well-defined, the upper index follows to

$$
j_{\max }=j_{\min }+i+m-1 .
$$

Hence, the function $\boldsymbol{\zeta}(t)$ in (B.11) must be evaluated at $i+m$ points, in order to approximate the $i$-th derivative $\mathrm{d}^{i} \boldsymbol{\zeta} / \mathrm{d} t^{i}$ with a desired error $O\left(h^{m}\right)$.

In view of the ODEs (B.1a), only the first derivative $\dot{\boldsymbol{\zeta}}(t)$ has to be approximated. Thereby, different finite-difference approximations can be calculated by means of (B.12). Table B.1 lists the coefficients $c_{j}$ for the approximation (B.11) of the first derivative $\dot{\boldsymbol{\zeta}}(t)$ in dependence of the lower index $j_{\min }$ and the approximation error $O\left(h^{m}\right)$.

The finite-difference formula (B.11) can be used to approximate the time derivative $\dot{\boldsymbol{\zeta}}(t)$ of the solution $\boldsymbol{\zeta}(t)$ at the time points $t_{i}$ with

$$
\hat{\boldsymbol{\zeta}}_{i}=\boldsymbol{\zeta}\left(t_{i}\right), \quad i=0,1, \ldots, N-1 \quad \text { and } \quad \hat{\boldsymbol{\zeta}}=\left[\boldsymbol{\zeta}_{0}, \ldots, \boldsymbol{\zeta}_{N-1}\right]
$$

The $\left(n_{\zeta} \times N\right)$-matrix $\hat{\boldsymbol{\zeta}}$ contains all states $\hat{\boldsymbol{\zeta}}_{i}$ corresponding to the respective time points $t_{i}$.

\begin{tabular}{|c|c|c|c|c|c|c|c|c|c|c|c|}
\hline$O\left(h^{m}\right)$ & $j_{\operatorname{mir}}$ & $\stackrel{(\mathrm{B} .13)}{\Longrightarrow} j_{\max }$ & $j:-4$ & -3 & -2 & -1 & 0 & 1 & 2 & 3 & 4 \\
\hline \multirow{2}{*}{$m=1$} & -1 & 0 & - & - & - & -1 & 1 & - & - & - & - \\
\hline & 0 & 1 & - & - & - & - & -1 & 1 & - & - & - \\
\hline \multirow[t]{3}{*}{$m=2$} & -2 & 0 & - & - & $\frac{1}{2}$ & -2 & $\frac{3}{2}$ & - & - & - & - \\
\hline & -1 & 1 & - & - & - & $-\frac{1}{2}$ & 0 & $\frac{1}{2}$ & - & - & - \\
\hline & 0 & 2 & - & - & - & - & $-\frac{3}{2}$ & 2 & $-\frac{1}{2}$ & - & - \\
\hline \multirow[t]{4}{*}{$m=3$} & -3 & 0 & - & $-\frac{1}{3}$ & $\frac{3}{2}$ & -3 & $\frac{11}{6}$ & - & - & - & - \\
\hline & -2 & 1 & - & - & $\frac{1}{6}$ & -1 & $\frac{1}{2}$ & $\frac{1}{3}$ & - & - & - \\
\hline & -1 & 2 & - & - & - & $-\frac{1}{3}$ & $-\frac{1}{2}$ & 1 & $-\frac{1}{6}$ & - & - \\
\hline & 0 & 3 & - & - & - & - & $-\frac{11}{6}$ & 3 & $-\frac{3}{2}$ & $\frac{1}{3}$ & - \\
\hline \multirow[t]{5}{*}{$m=4$} & -4 & 0 & $\frac{1}{4}$ & $-\frac{4}{3}$ & 3 & -4 & $\frac{25}{12}$ & - & - & - & - \\
\hline & -3 & 1 & - & $-\frac{1}{12}$ & $\frac{1}{2}$ & $-\frac{3}{2}$ & $\frac{5}{6}$ & $\frac{1}{4}$ & - & - & - \\
\hline & -2 & 2 & - & - & $\frac{1}{12}$ & $-\frac{2}{3}$ & 0 & $\frac{2}{3}$ & $-\frac{1}{12}$ & - & - \\
\hline & -1 & 3 & - & - & - & $-\frac{1}{4}$ & $-\frac{5}{6}$ & $\frac{3}{2}$ & $-\frac{1}{2}$ & $\frac{1}{12}$ & - \\
\hline & 0 & 4 & - & - & - & - & $-\frac{25}{12}$ & 4 & -3 & $\frac{4}{3}$ & $-\frac{1}{4}$ \\
\hline
\end{tabular}
The coefficients $c_{j}$ in (B.11) can be comprised in a differentiation matrix $D$ such that the time

Table B.1: Coefficients $c_{j}$ for the finite-difference approximation (B.11) of the first derivative $\dot{\boldsymbol{\zeta}}(t)$ for different approximation errors $O\left(h^{m}\right)$ and indices $j_{\min }$ (Wieland, 2005). 
derivative $\dot{\boldsymbol{\zeta}}(t)$ is approximated by

$$
\frac{\partial \boldsymbol{\zeta}}{\partial t}\left(t_{i}\right) \approx \hat{\boldsymbol{\zeta}}_{i}^{\prime} \quad \text { with } \quad \hat{\boldsymbol{\zeta}}^{\prime}=\left[D \hat{\boldsymbol{\zeta}}^{\top}\right]^{\top}=\hat{\boldsymbol{\zeta}} D^{\top} .
$$

In order to construct the differentiation matrix $D$, the differentiation formula (B.11) can be used at all grid points $t_{i}$ with the exception of a few points near the boundaries $t_{0}=0$ and $t_{N}=T$ in order to avoid the introduction of fictitious grid points outside the considered interval $[0, T]$. The exact number depends on the values of $j_{\min }$ and $j_{\max }$. For instance, if central differences with the approximation error $O\left(h^{2}\right)$ are used $\left(j_{\min }=-1\right.$, see Table B.1), then the differentiation formula with the same error $O\left(h^{2}\right)$ but with $j_{\min }=0$ for the first point $t_{0}$ and $j_{\min }=-2\left(j_{\max }=0\right)$ for the final point $t_{N}$ can be used. For this specific example, the differentiation matrix $D$ has the structure

$$
D=\frac{1}{2 h}\left[\begin{array}{rrrrr}
-3 & 4 & -1 & & \\
-1 & 0 & 1 & & \\
& \ddots & & \ddots & \\
& & -1 & 0 & 1 \\
& & 1 & -4 & 3
\end{array}\right]
$$

Replacing the left-hand side of the ODEs (B.1a) with the finite-difference approximation (B.15) results in the algebraic equations

$$
\hat{\boldsymbol{\zeta}}^{\prime}=\boldsymbol{f}\left(\hat{\boldsymbol{\zeta}}, t_{i}, \boldsymbol{p}\right), \quad i=1, \ldots, N .
$$

Note that the index $i$ starts at $i=1$ instead of $i=0$ in order to incorporate the BCs (B.1b):

$$
\boldsymbol{g}\left(\hat{\boldsymbol{\zeta}}_{0}, \hat{\boldsymbol{\zeta}}_{N-1}, \boldsymbol{p}\right)=\mathbf{0}
$$

In this way and in view of (B.2), the $N n_{\zeta}+n_{g}$ equations in (B.17) equal the number of $(N+1) n_{\zeta}+n_{p} \stackrel{(\mathrm{B} .2)}{=} N n_{\zeta}+n_{g}$ unknown states $\hat{\boldsymbol{\zeta}}_{i}$ and free parameters $\boldsymbol{p}$. The equation set (B.17) can be iteratively solved e.g. with Newton's method.

Remark B.2. A simple way to avoid programming Newton's method for solving the nonlinear algebraic equations (B.17) is to use the MATLAB function fsolve of the Optimization Toolbox. For several considered examples, the function fsolve has shown excellent numerical robustness and accuracy for the multi-step finite-difference schemes, even if the analytical Jacobians $\partial \boldsymbol{f} / \partial \boldsymbol{\zeta}$ and $\partial \boldsymbol{f} / \partial \boldsymbol{p}$ were not provided.

\section{B.3 Shooting method}

In contrast to the collocation and finite-difference methods which solve an algebraic set of equations for both the ODE and BCs in (B.1), the shooting method splits the solution of the ODEs and BCs in two parts. In a first step, the initial value problem (IVP) corresponding to the BVP (B.1) is defined as

$$
\dot{\boldsymbol{\zeta}}=\boldsymbol{f}(\boldsymbol{\zeta}, t, \boldsymbol{p}), \quad \boldsymbol{\zeta}(0)=\boldsymbol{\zeta}_{0}
$$


in dependence of the initial condition $\boldsymbol{\zeta}_{0}$ and the free parameters $\boldsymbol{p}$. The solution of the IVP (B.18) can be formally written as

$$
\boldsymbol{\zeta}\left(\boldsymbol{\zeta}_{0}, \boldsymbol{p} ; t\right)=\boldsymbol{\zeta}_{0}+\int_{0}^{t} \boldsymbol{f}(\boldsymbol{\zeta}(\tau), \tau, \boldsymbol{p}) \mathrm{d} \tau
$$

whereby the initial condition $\boldsymbol{\zeta}_{0}$ and the free parameters $\boldsymbol{p}$ have to be determined such that $\boldsymbol{\zeta}\left(\boldsymbol{\zeta}_{0}, \boldsymbol{p} ; t\right)$ is a solution of the BVP (B.1). Hence, in a second step, the BCs (B.1b) lead to $n_{g}$ algebraic equations

$$
\boldsymbol{g}\left(\boldsymbol{\zeta}_{0}, \boldsymbol{\zeta}\left(\boldsymbol{\zeta}_{0}, \boldsymbol{p} ; T\right), \boldsymbol{p}\right)=\mathbf{0}
$$

for the same number of initial states $\boldsymbol{\zeta}_{0}$ and the free parameters $\boldsymbol{p}$, cf. Condition (B.2).

With respect to the finite-time transition problems treated in this thesis, the general nonlinear BCs (B.1b) can be split in separate BCs $\boldsymbol{\zeta}(0)=\boldsymbol{\zeta}_{0}$ and $\boldsymbol{\zeta}(T)=\boldsymbol{\zeta}_{T}$ with the known initial and terminal states $\boldsymbol{\zeta}_{0}$ and $\boldsymbol{\zeta}_{T}$. Since the integration (B.19) is started with the correct initial state $\boldsymbol{\zeta}_{0}$, the equations (B.20) reduce to the $n_{\zeta}$ terminal conditions

$$
\boldsymbol{\zeta}\left(\boldsymbol{\zeta}_{0}, \boldsymbol{p} ; T\right)-\boldsymbol{\zeta}_{T}=0
$$

for the $n_{\zeta}$ free parameters $\boldsymbol{p}$, which can be iteratively solved with Newton's method.

In general, the solution $\boldsymbol{\zeta}\left(\boldsymbol{\zeta}_{0}, \boldsymbol{p} ; t\right)$ of the nonlinear IVP (B.18) requires numerical integration with an appropriate integration scheme. This directly shows the main problem of the shooting method compared to collocation or finite-difference schemes: for unstable and highly nonlinear ODEs (e.g. the double pendulum swing-up in Section 2.4), the numerical integration of (B.18) may be ill-conditioned. Moreover, the sensitivity matrix $\partial \boldsymbol{\zeta} / \partial \boldsymbol{p}$ at $t=T$ required for the numerical solution of (B.21) cannot be provided analytically for most nonlinear systems and therefore has to be approximated numerically. ${ }^{3}$ In contrast to this, the Jacobians $\partial \boldsymbol{f} / \partial \boldsymbol{\zeta}$ and $\partial \boldsymbol{f} / \partial \boldsymbol{p}$ which are required by collocation and finite-difference schemes can be derived analytically in most cases.

\footnotetext{
${ }^{3}$ In order to solve the algebraic equation (B.21) with respect to $\boldsymbol{p}$ requires the sensitivity matrix $\partial \boldsymbol{\zeta} / \partial \boldsymbol{p}$ at $t=T$ to have full rank. To check this rank condition analytically is practically impossible for general nonlinear systems. As pointed out in Section 2.3.1, this problem can be seen in correspondence to the fact that the analytic investigation of controllability of nonlinear systems is still an unsolved problem.
} 


\section{Appendix C}

\section{Incorporation of output constraints with non-asymptotic saturation functions}

As explained in Chapter 4, the constraints (4.1) on the output trajectory $y^{*}(t)$ are systematically incorporated in the feedforward control design by constructing the new dynamic system (4.9). For the sake of simplicity, the first $r$ saturation functions $\psi_{i}\left(\xi_{i}, \psi_{i}^{ \pm}\left(\boldsymbol{\xi}_{i-1}\right)\right), i=1, \ldots, r-$ with $\boldsymbol{\xi}_{0}=\emptyset$ and $\boldsymbol{\xi}_{1}=\xi_{1}$ as defined in (4.10) — are assumed to be smooth and strictly monotonically increasing (see Condition (4.11) and Figure 4.1). This assumption simplifies the construction of the dynamic system (4.9) and the calculation of the saturation limits (4.17). Moreover, the smooth saturation functions can be easily realized by means of the setup (4.12).

However, the system (4.9) for the constrained output $y^{*}(t)$ is also valid for non-asymptotic saturation functions $\psi_{i}\left(\xi_{i}, \psi_{i}^{ \pm}\left(\boldsymbol{\xi}_{i-1}\right)\right), i=1, \ldots, r$ with

$$
\frac{\partial \psi_{i}}{\partial \xi_{i}} \geq 0, \quad \psi_{i}^{+} \leq \psi_{i} \leq \psi_{i}^{+}, \quad 1, \ldots, r
$$

such that the saturation limits $\psi_{i}^{ \pm}\left(\boldsymbol{\xi}_{i-1}\right)$ and correspondingly the output constraints (4.1) are exactly fulfilled. ${ }^{1}$ In the next sections, the saturation limits (4.17) as well as the definition of the saturation functions $\psi_{i}\left(\xi_{i}, \psi_{i}^{ \pm}\left(\boldsymbol{\xi}_{i-1}\right)\right), i=1, \ldots, r$ are adapted to the non-asymptotic case.

\section{C.1 Calculation of non-asymptotic saturation limits}

The monotonicity condition (4.11) for the asymptotic saturation functions (4.12) ensures that the denominator $\prod_{j=1}^{i} \partial \psi_{j} / \partial \xi_{j}$ of the saturation limits (4.17) is greater than zero. In view of

\footnotetext{
${ }^{1}$ In case of the asymptotic limits $\psi_{i}^{ \pm}\left(\boldsymbol{\xi}_{i-1}\right), i=1, \ldots, r$ of the saturation functions (4.12), also the output constraints (4.1) for the output $y^{*}$ and its first $r-1$ derivatives $y^{*(i)}, i=1, \ldots, r-1$ in (4.14) are only reached asymptotically. However, this effect is almost negligible, see e.g. Figure 4.4 and 4.7.
} 
(C.1), the gradients $\partial \psi_{i} / \partial \xi_{i}$ of the non-asymptotic saturation functions can be exactly zero. Hence, the definition (4.17) of the saturation limits has to be adapted accordingly:

$$
\psi_{1}^{ \pm}=y_{0}^{ \pm}, \quad \psi_{i+1}^{ \pm}\left(\boldsymbol{\xi}_{i}\right)=\left\{\begin{array}{cc}
\frac{y_{i}^{ \pm}-\gamma_{i}\left(\boldsymbol{\xi}_{i}\right)}{i} & \text { if } \prod_{j=1}^{i} \frac{\partial \psi_{j}}{\partial \xi_{j}} \neq 0 \\
\prod_{j=1} \frac{\partial \psi_{j}}{\partial \xi_{j}} & \text { if } \prod_{j=1}^{i} \frac{\partial \psi_{j}}{\partial \xi_{j}}=0 \\
\pm \infty & \quad i=1, \ldots, r .
\end{array}\right.
$$

It is proved in Section 4.1.3 that the saturation limits (C.2a) for $\prod_{j=1}^{i} \partial \psi_{j} / \partial \xi_{j} \neq 0$ ensure that the output constraints for $y^{*(i)} \in\left[y_{i}^{-}, y_{i}^{+}\right], i=1, \ldots, r$ are satisfied $\left(y^{*} \in\left[y_{0}^{-}, y_{0}^{+}\right]\right.$is directly fulfilled by $\left.\psi_{1}^{ \pm}=y_{0}^{ \pm}\right)$. However, if $\prod_{j=1}^{i} \partial \psi_{j} / \partial \xi_{j}=0$ holds in the second case $(\mathrm{C} .2 \mathrm{~b})$, then the corresponding output derivative $y^{*(i)}$ in (4.14) cannot be influenced by the saturation function $\psi_{i+1}\left(\cdot, \psi_{i+1}^{ \pm}\left(\boldsymbol{\xi}_{i}\right)\right.$ ) (with the argument $(\cdot)$ given by $\xi_{i+1}$ or $v$ for $i=r$, respectively). In order to prove that for $\prod_{j=1}^{i} \partial \psi_{j} / \partial \xi_{j}=0$ the output constraints (4.1) are automatically satisfied, the following lemma is required:

Lemma C.1. If there exists a smallest index $k \in\{1, \ldots, r\}$ such that $\partial \psi_{k} / \partial \xi_{k}=0$, then $y^{*(i)}=0$ holds for the output derivatives with $i=k, \ldots, r$.

Proof. If $\partial \psi_{k} / \partial \xi_{k}=0$ holds, then $\psi_{k}\left(\xi_{k}, \psi_{k}^{ \pm}\left(\boldsymbol{\xi}_{k-1}\right)\right)$ is in saturation either at $\psi_{k}^{-}\left(\boldsymbol{\xi}_{k-1}\right)$ or $\psi_{k}^{+}\left(\boldsymbol{\xi}_{k-1}\right)$. This implies in view of $(\mathrm{C} .2 \mathrm{a})$ that

$$
\psi_{k}\left(\xi_{k}, \psi_{k}^{ \pm}\left(\boldsymbol{\xi}_{k-1}\right)\right)=\psi_{k}^{ \pm}\left(\boldsymbol{\xi}_{k-1}\right)=\frac{y_{k-1}^{ \pm}-\gamma_{k-1}\left(\boldsymbol{\xi}_{k-1}\right)}{\prod_{j=1}^{k-1} \frac{\partial \psi_{j}}{\partial \xi_{j}}}
$$

holds, since $\prod_{j=1}^{k-1} \partial \psi_{j} / \partial \xi_{j} \neq 0$ follows from the assumption that all saturation functions $\psi_{j}$ with $j<k$ are not in saturation. Moreover, placing (C.3) in (4.14) reveals that the corresponding output derivative

$$
y^{*(k-1)}=\gamma_{k-1}\left(\boldsymbol{\xi}_{k-1}\right)+\prod_{j=1}^{k-1} \frac{\partial \psi_{j}}{\partial \xi_{j}} \cdot \psi_{k}\left(\xi_{k}, \psi_{k}^{ \pm}\left(\boldsymbol{\xi}_{k-1}\right)\right) \stackrel{(\mathrm{C} .3)}{=} y_{k-1}^{ \pm}=\text {const } .
$$

stays exactly at the constraint $y_{k-1}^{-}$or $y_{k-1}^{+}$. Hence, the higher output derivatives $y^{*(i)}$ with $i>k$ are zero as stated in the lemma.

Lemma C.1 can be used straightforwardly to verify that the output constraints (4.1) are not violated by $\prod_{j=1}^{i} \partial \psi_{j} / \partial \xi_{j}=0$ in (C.2b), i.e. a certain saturation function is given by $\partial \psi_{j} / \partial \xi_{j}=$ $0, j \in\{1, \ldots, i\}$. In this case, Lemma C.1 states that $y^{*(i)}=0$ for $i=j, \ldots, r$, which lies inside the respective constraints $\left[y_{i}^{-}, y_{i}^{+}\right]$due to the condition (4.2).

The following corollary directly follows from Lemma C.1 and is required in the next section:

Corollary C.1. If $y^{*(i)} \neq 0$ holds for a certain output derivative $y^{*(i)}, i \in\{1, \ldots, r\}$ defined in (4.14), then $\prod_{j=1}^{i} \partial \psi_{j} / \partial \xi_{j} \neq 0$ in (C.2) is ensured. 


\section{C.2 Choice of non-asymptotic saturation functions}

The construction of the non-asymptotic saturation functions $\psi_{i}\left(\xi_{i}, \psi_{i}^{ \pm}\left(\boldsymbol{\xi}_{i-1}\right)\right), i=1, \ldots, r$ has to account for the case-dependent definition (C.2) in order to avoid a division by zero. In a first step, the last ramp-shaped saturation function $\psi_{r+1}\left(v, \psi_{r+1}^{ \pm}\left(\boldsymbol{\xi}_{r}\right)\right)$ is adapted to the non-asymptotic case, before the more complicated design of the first $r$ saturation functions $\psi_{i}\left(\xi_{i}, \psi_{i}^{ \pm}\left(\boldsymbol{\xi}_{i-1}\right)\right), i=1, \ldots, r$ is addressed.

\section{Ramp-shaped saturation function $\psi_{r+1}\left(v, \psi_{r+1}^{ \pm}\left(\boldsymbol{\xi}_{r}\right)\right)$}

The last saturation function $\psi_{r+1}\left(v, \psi_{r+1}^{ \pm}\left(\boldsymbol{\xi}_{r}\right)\right)$ given in (4.13) is redefined according to

$$
\psi_{r+1}\left(v, \psi_{r+1}^{ \pm}\left(\boldsymbol{\xi}_{r}\right)\right)= \begin{cases}v & \text { if } y_{v}^{*(r)} \in\left[y_{r}^{-}, y_{r}^{+}\right] \\ \psi_{r+1}^{-}\left(\boldsymbol{\xi}_{r}\right) & \text { if } y_{v}^{*(r)}<y_{r}^{-} \\ \psi_{r+1}^{+}\left(\boldsymbol{\xi}_{r}\right) & \text { if } y_{v}^{*(r)}>y_{r}^{+}\end{cases}
$$

with

$$
y_{v}^{*(r)}=\gamma_{r}\left(\boldsymbol{\xi}_{r}\right)+\prod_{j=1}^{r} \frac{\partial \psi_{j}}{\partial \xi_{j}} \cdot v
$$

following from (4.14) with $i=r$ if the new input $v$ is used as the saturation function $\psi_{r+1}=v$. If $y_{v}^{*(r)}$ lies within the respective constraints $\left[y_{r}^{-}, y_{r}^{+}\right]$, then $\psi_{r+1}=v$ holds, see (C.5a). Otherwise, the respective limits $\psi_{r+1}^{-}\left(\boldsymbol{\xi}_{r}\right)$ or $\psi_{r+1}^{+}\left(\boldsymbol{\xi}_{r}\right)$ determined in (C.2a) are used for $\psi_{r+1}\left(v, \psi_{r+1}^{ \pm}\left(\boldsymbol{\xi}_{r}\right)\right)$. In these cases (C.5b) and (C.5c), the highest output derivative $y^{*(r)}$ is set to one of the constraints $y_{r}^{-}$or $y_{r}^{+}$which are away from zero, i.e. $y_{r}^{ \pm} \gtrless 0$ due to Condition (4.2). Hence, according to Corollary C.1, $\prod_{j=1}^{r} \partial \psi_{j} / \partial \xi_{j} \neq 0$ holds and the limits $\psi_{r+1}^{ \pm}\left(\boldsymbol{\xi}_{r}\right)$ in (C.2a) can be determined without dividing by zero.

Non-asymptotic saturation functions $\psi_{i}\left(\xi_{i}, \psi_{i}^{ \pm}\left(\boldsymbol{\xi}_{i-1}\right)\right), i=1, \ldots, r$

The design of the first $r$ saturation functions $\psi_{i}\left(\xi_{i}, \psi_{i}^{ \pm}\left(\boldsymbol{\xi}_{i-1}\right)\right), i=1, \ldots, r$ is more complicated. In order to guarantee continuity of the highest output derivative $y^{*(r)}$ in $(4.14)$, the single functions $\psi_{i}\left(\xi_{i}, \psi_{i}^{ \pm}\left(\boldsymbol{\xi}_{i-1}\right)\right)$ have to be $\mathcal{C}^{r-i+1}$-continuous. Therefore, $\psi_{i}\left(\xi_{i}, \psi_{i}^{ \pm}\left(\boldsymbol{\xi}_{i-1}\right)\right)$ is defined in five parts ${ }^{2}$

$$
\psi_{i}\left(\xi_{i}, \psi_{i}^{ \pm}\left(\boldsymbol{\xi}_{i-1}\right)\right)= \begin{cases}\xi_{i} & \text { if } y_{\xi_{i}}^{*(i-1)} \in\left[y_{i-1}^{-}+\epsilon_{0} \frac{\Delta y_{i-1}^{ \pm}}{2}, y_{i-1}^{+}-\epsilon_{0} \frac{\Delta y_{i-1}^{ \pm}}{2}\right] \\ g_{r-i+1}\left(\xi_{i}, \delta \boldsymbol{g}_{i}^{-}, \boldsymbol{g}_{i}^{-}\right) & \text {if } \xi_{i} \in\left(\xi_{i}^{-}, \delta \xi_{i}^{-}\right) \\ g_{r-i+1}\left(\xi_{i}, \delta \boldsymbol{g}_{i}^{+}, \boldsymbol{g}_{i}^{+}\right) & \text {if } \xi_{i} \in\left(\delta \xi_{i}^{+}, \xi_{i}^{+}\right) \\ \psi_{i}^{-} & \text {if } \xi_{i} \leq \xi_{i}^{-} \\ \psi_{i}^{+} & \text {if } \xi_{i} \geq \xi_{i}^{+}\end{cases}
$$

\footnotetext{
${ }^{2}$ The argument $\boldsymbol{\xi}_{i-1}$ of the saturation limits $\psi_{i}^{ \pm}\left(\boldsymbol{\xi}_{i-1}\right)$ is omitted to not further complicate the notation.
} 


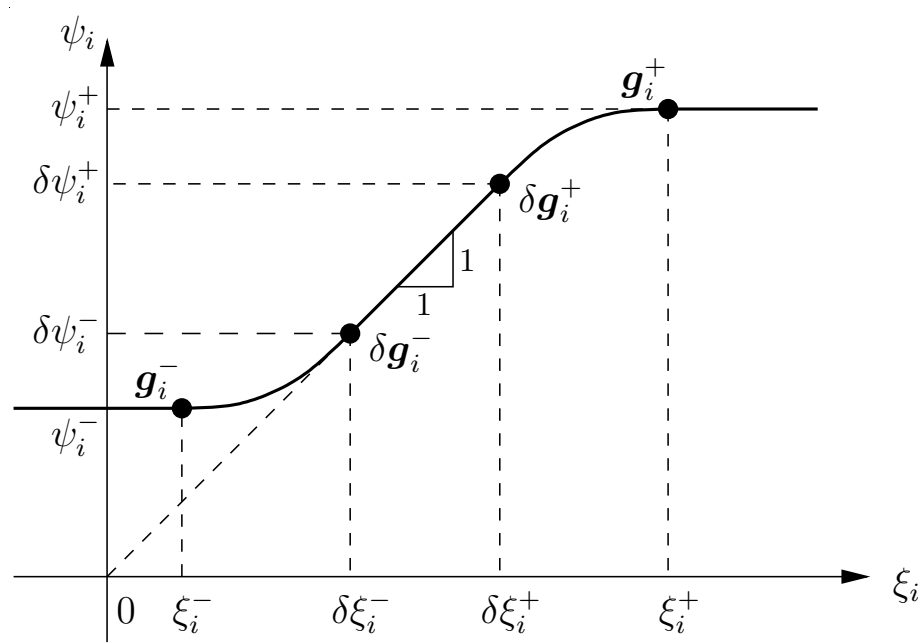

Figure C.1: Construction of the non-asymptotic saturation functions $\psi_{i}\left(\xi_{i}, \psi_{i}\left(\boldsymbol{\xi}_{i-1}\right)\right), i=1, \ldots, r$ in five parts with the intersection points $\delta \boldsymbol{g}_{i}^{ \pm}=\left(\delta \xi_{i}^{ \pm}, \delta \psi_{i}^{ \pm}\right)$and $\boldsymbol{g}_{i}^{ \pm}=\left(\xi_{i}^{ \pm}, \psi_{i}^{ \pm}\right)$.

with $\Delta y_{i-1}^{ \pm}=y_{i-1}^{+}-y_{i-1}^{-}$and

$$
y_{\xi_{i}}^{*(i-1)}=\gamma_{i-1}\left(\boldsymbol{\xi}_{i-1}\right)+\prod_{j=1}^{i-1} \frac{\partial \psi_{j}}{\partial \xi_{j}} \cdot \xi_{i}, \quad i=1, \ldots, r .
$$

Figure C.1 illustrates this setup. The saturation function is given by $\psi_{i}\left(\xi_{i}, \psi_{i}\left(\boldsymbol{\xi}_{i-1}\right)\right)=\xi_{i}$ if the corresponding output derivative (C.8) following from (4.14) lies within a specified tolerance interval $y_{\xi_{i}}^{*(i-1)} \in\left[y_{i-1}^{-}+\epsilon_{0} \frac{\Delta y_{i-1}^{ \pm}}{2}, y_{i-1}^{+}-\epsilon_{0} \frac{\Delta y_{i-1}^{ \pm}}{2}\right]$. Thereby, $\epsilon_{0} \in(0,1)$ is a design parameter which determines the length of the interval where the saturation function is linear, see Figure C.1. At the boundaries $y_{\xi_{i}}^{*(i-1)}=y_{i-1}^{-}+\epsilon_{0} \frac{\Delta y_{i-1}^{ \pm}}{2}$ and $y_{\xi_{i}}^{*(i-1)}=y_{i-1}^{+}-\epsilon_{0} \frac{\Delta y_{i-1}^{ \pm}}{2}$, the saturation function is given by $\psi_{i}\left(\xi_{i}, \psi_{i}\left(\boldsymbol{\xi}_{i-1}\right)=\delta \psi_{i}^{ \pm}\left(\boldsymbol{\xi}_{i-1}\right)\right.$ with

$$
\delta \psi_{1}^{ \pm}=y_{0}^{ \pm} \mp \epsilon_{0} \frac{\Delta y_{0}^{ \pm}}{2}, \quad \delta \psi_{i}^{ \pm}\left(\boldsymbol{\xi}_{i-1}\right)=\frac{y_{i-1}^{ \pm} \mp \epsilon_{0} \frac{\Delta y_{i-1}^{ \pm}}{2}-\gamma_{i-1}\left(\boldsymbol{\xi}_{i-1}\right)}{\prod_{j=1}^{i-1} \frac{\partial \psi_{j}}{\partial \xi_{j}}}=\delta \xi_{i}^{ \pm} \quad i=2, \ldots, r .
$$

Note that in view of Corollary C.1, $\prod_{j=1}^{i-1} \partial \psi_{j} / \partial \xi_{j} \neq 0$ holds for $i=2, \ldots, r$ because $y_{\xi_{i}}^{*(i-1)}=$ $\epsilon_{0} y_{i-1}^{ \pm} \neq 0$ for $\epsilon_{0} \in(0,1)$.

The transition between the points $\delta \boldsymbol{g}_{i}^{-}$and $\boldsymbol{g}_{i}^{-}$or respectively between $\delta \boldsymbol{g}_{i}^{+}$and $\boldsymbol{g}_{i}^{+}$(see Figure C.1) is achieved via the interpolation function $g_{k}\left(\xi_{i}, \delta \boldsymbol{g}_{i}^{ \pm}, \boldsymbol{g}_{i}^{ \pm}\right)$in (C.7b) and (C.7c). The index $k$ denotes the required differentiability of $g_{k}$. As mentioned above, the saturation functions (C.7) have to be $\mathcal{C}^{r-i+1}$-continuous to ensure continuity of the highest output derivative $y^{*(r)}$ in (4.14). Hence, $k=r-i+1$ holds for the interpolation function $g_{k}\left(\xi_{i}, \delta \boldsymbol{g}_{i}^{ \pm}, \boldsymbol{g}_{i}^{ \pm}\right)$. For the sake of simplicity, $g_{k}$ is constructed for the lower points $\boldsymbol{g}_{i}^{-}$and $\boldsymbol{g}_{i}^{-}$in (C.7b). ${ }^{3}$ To achieve $\mathcal{C}^{k}$ continuity of $g_{k}\left(\xi_{i}, \delta \boldsymbol{g}_{i}^{-}, \boldsymbol{g}_{i}^{-}\right)$with respect to the points $\boldsymbol{g}_{i}^{-}$and $\boldsymbol{g}_{i}^{-}$in Figure C.1, the following

\footnotetext{
${ }^{3}$ To obtain $g_{k}\left(\xi_{i}, \delta \boldsymbol{g}_{i}^{+}, \boldsymbol{g}_{i}^{+}\right)$in the case (C.7c), the arguments $(\cdot)^{-}$have to be replaced by $(\cdot)^{+}$.
} 
$2(k+1)$ BCs have to be satisfied:

$$
\begin{aligned}
& g_{k}\left(\delta \xi_{i}^{-}, \delta \boldsymbol{g}_{i}^{-}, \boldsymbol{g}_{i}^{-}\right)=\delta \psi_{i}^{-}, \quad g_{k}\left(\xi_{i}^{-}, \delta \boldsymbol{g}_{i}^{-}, \boldsymbol{g}_{i}^{-}\right)=\psi_{i}^{-},\left.\quad \frac{\partial g_{k}}{\partial \xi_{i}}\right|_{\xi_{i}=\delta \xi_{i}^{-}}=1,\left.\quad \frac{\partial g_{k}}{\partial \xi_{i}}\right|_{\xi_{i}=\xi_{i}^{-}}=0 \\
& \left.\frac{\partial^{j} g_{k}}{\partial \xi_{i}^{j}}\right|_{\xi_{i}=\delta \xi_{i}^{-}}=\left.\frac{\partial^{j} g_{k}}{\partial \xi_{i}^{j}}\right|_{\xi_{i}=\xi_{i}^{-}}=0, \quad j=2, \ldots, k \quad \text { if } \quad k>1 .
\end{aligned}
$$

\section{Construction of interpolation function $g_{k}\left(\xi_{i}, \delta \boldsymbol{g}_{i}^{ \pm}, \boldsymbol{g}_{i}^{ \pm}\right)$}

A polynomial is used to construct $g_{k}\left(\xi_{i}, \delta \boldsymbol{g}_{i}^{-}, \boldsymbol{g}_{i}^{-}\right)$exemplarily for the case (C.7b):

$$
g_{k}\left(\xi_{i}, \delta \boldsymbol{g}_{i}^{-}, \boldsymbol{g}_{i}^{-}\right)=\sum_{j=0}^{2 k+1} a_{j}\left(\frac{\xi_{i}-\delta \xi_{i}^{-}}{\Delta \xi_{i}^{-}}\right)^{j}, \quad \xi_{i} \in\left(\xi^{-}, \delta \xi_{i}^{-}\right)
$$

with $\Delta \xi_{i}^{-}=\xi_{i}^{-}-\delta \xi_{i}^{-}$. The coefficients $a_{j}, j=0, \ldots, 2 k+1$ have to be determined such that the boundary conditions (C.10) are satisfied. As shown in Figure C.1, the inner boundary $\delta \xi_{i}^{-}$ is simply given by

$$
\delta \xi_{i}^{-}=\delta \psi_{i}^{-}
$$

with $\delta \psi_{i}^{-}$following from (C.9). The choice of the outer boundary $\xi_{i}^{-}$is a degree of freedom for the interpolation function $g_{k}\left(\xi_{i}, \delta \boldsymbol{g}_{i}^{-}, \boldsymbol{g}_{i}^{-}\right)$. Therefore, a new design parameter $\epsilon_{1}$ is introduced to determine $\xi_{i}^{-}$:

$$
\underbrace{\left(\xi_{i}^{-}-\delta \xi_{i}^{-}\right)}_{=\Delta \xi_{i}^{-}} \stackrel{!}{=} \underbrace{\epsilon_{1}\left(\psi_{i}^{-}-\delta \psi_{I}^{-}\right)}_{=\Delta \psi_{i}^{-}} \quad \stackrel{(\mathrm{C} .12 \mathrm{a})}{\Longrightarrow} \quad \xi_{i}^{-}=\delta \psi_{i}^{-}+\epsilon_{1} \Delta \psi_{i}^{-} .
$$

Hence, the design parameter $\epsilon_{1}$ determines the length of the intervals $\left(\xi_{i}^{-}, \delta \xi_{i}^{-}\right)$until the saturation limit $\psi_{i}^{-}$is reached, see Figure C.1. The $k+1$ BCs in (C.10) for $\xi_{i}=\delta \xi_{i}^{-}$and the relations (C.12) simplify the polynomial structure (C.11) to

$$
g_{k}\left(\xi_{i}, \delta \boldsymbol{g}_{i}^{-}, \boldsymbol{g}_{i}^{-}\right)=\delta \xi_{i}^{-}+\sum_{j=k+1}^{2 k+1} a_{j}\left(\frac{\xi_{i}-\delta \psi_{i}^{-}}{\epsilon_{1} \Delta \psi_{i}^{-}}\right)^{j}, \quad \xi_{i} \in\left(\xi^{-}, \delta \xi_{i}^{-}\right)
$$

Table C. 1 shows the remaining coefficients $a_{j}, j=k+2, \ldots, 2 k+1$ for the polynomials (C.13) with $k=1, \ldots, 4$.

Furthermore, the design parameter $\epsilon_{1}$ has to be chosen such that $g_{k}\left(\xi_{i}, \delta \boldsymbol{g}_{i}^{-}, \boldsymbol{g}_{i}^{-}\right)$is monotonic and contains no undershoot or overshoot which would violate the limit $\psi_{i}^{-}$. This can be achieved by enforcing

$$
\left.\frac{\partial^{k+1} g_{k}}{\partial \xi_{i}^{k+1}}\right|_{\xi_{i}^{-}} \stackrel{!}{\geq} 0
$$

Evaluating the condition (C.14) for the polynomial (C.13) results in an inequality for $\epsilon_{1}$, which has to be satisfied to ensure the monotonicity of $g_{k}\left(\xi_{i}, \delta \boldsymbol{g}_{i}^{-}, \boldsymbol{g}_{i}^{-}\right)$, see Table C.1 for $k=1, \ldots, 4$. Both design parameters $\epsilon_{0}$ and $\epsilon_{1}$ influence the shape of the saturation functions (C.7), also see Figure C.1. The linearity interval of $\psi_{i}\left(\xi_{i}, \psi_{i}^{ \pm}\left(\boldsymbol{\xi}_{i-1}\right)\right)$ can be influenced by $\epsilon_{0}$, whereas the second parameter $\epsilon_{1}$ adjusts the intervals until the saturation limits $\psi_{i}^{ \pm}\left(\boldsymbol{\xi}_{i-1}\right)$ are reached. 


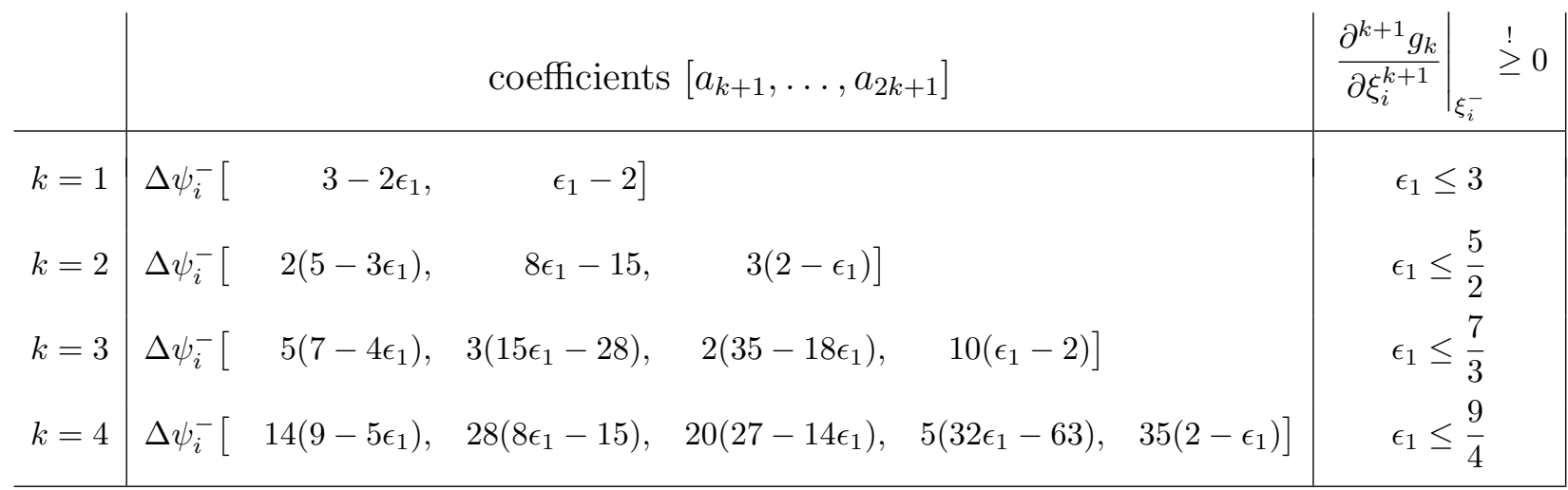

Table C.1: Coefficients $a_{j}, j=k+1 \ldots, 2 k+1$ of the $\mathcal{C}^{k}$-continuous polynomial $g_{k}\left(\xi_{i}, \delta \boldsymbol{g}_{i}^{-}, \boldsymbol{g}_{i}^{-}\right)$in (C.13) for $k=1, \ldots, 4$.

\section{C.3 Conclusions}

The main intention of this appendix is to show that non-asymptotic saturation functions can be used for the dynamic system (4.9) derived in Chapter 4, such that the output trajectory $y^{*}(t)$ exactly (and not asymptotically) fulfills the constraints (4.1). In order to avoid a division by zero, the definition of the saturation functions (C.5) and (C.7) ensures that the saturation limits (C.2a) are only evaluated if no saturation function $\psi_{i}$ and its corresponding output variable $y^{*(i-1)}$ are in saturation.

However, serious problems occur if the input constraints are incorporated in the feedforward control design according to Section 4.3. The evaluation of the redefined function $\widehat{\psi}_{r+1}$ in (4.33) will lead to a division by zero if an output derivative is constrained by $y^{*(i)}=y_{i}^{ \pm}$, while at the same time one of the projected output constraints (4.36) crosses zero, i.e. $y_{r}^{-}>0$ or $y_{r}^{+}<0$. In this case, the asymptotic saturation functions (4.12) have distinct numerical advantages, since the denominator $\prod_{j=1}^{r} \partial \psi_{j} / \partial \xi_{j}$ in (4.33) only approaches (instead of being identically) zero, cf. Example 4.2.

Moreover, the non-asymptotic saturation functions (C.7) require a high implementation effort due to the case-dependent definition in five parts, which also increases the complexity of the partial derivatives occurring in (C.2a) and (C.8). In contrast to this, the asymptotic saturation functions in (4.12) have a simple structure and are naturally smooth. Moreover, their asymptotic nature is usually negligible, see e.g. Figure 4.4 and 4.7. 


\section{Bibliography}

S.K. Agrawal, N. Faiz, and R.M. Murray. Feasible trajectories of linear dynamic systems with inequality constraints using higher-order representations. In Proc. 15th IFAC World Congress, pages 139-144, Beijing, China, July 1999.

M.J. Anderson and W.J. Grantham. Lyapunov optimal feedback control of a nonlinear inverted pendulum. Journal of Dynamic Systems, Measurement, and Control, 111:554-558, 1989.

T.M. Apostol. Mathematical analysis. Addison-Wesley, Reading, Massachusetts, 2nd edition, 1974.

U.M. Ascher, R.M.M. Mattheij, and R.D. Russell. Numerical solution of boundary value problems of ordinary differential equations. Prentice Hall, 1988.

K.J. Åström and K. Furuta. Swinging up a pendulum by energy control. Automatica, 36:287-295, 2000 .

W. Auzinger, O. Koch, and E. Weinmüller. Efficient collocation schemes for singular boundary value problems. Numerical Algorithms, 31:5-25, 2002.

W. Auzinger, G. Kneisl, O. Koch, and E. Weinmüller. SBVP 1.0 - A Matlab solver for singular boundary value problems, 2003a. Technical Report 2/02 (unpublished), Institute for Applied Mathematics and Numerical Analysis, Vienna University of Technology, http://www.math.tuwien.ac.at/ ewa/.

W. Auzinger, O. Koch, J. Petrickovic, and E. Weinmüller. Numerical solution of boundary value problems with an essential singularity, 2003b. Technical Report 3/03 (unpublished), Institute for Applied Mathematics and Numerical Analysis, Vienna University of Technology, http://www.math.tuwien.ac.at/ inst115/preprints.htm.

P.B. Bailey, L.F. Shampine, and P.E. Waltman. Nonlinear two point boundary value problems. Academic Press, New York, 1968.

M. Benosman and G. Le Vey. Stable inversion of SISO nonminimum phase linear systems through output planning: an experimental application to the one-link flexible manipulator. IEEE Transactions on Control Systems Technology, 11:588-597, 2003.

M. Benosman, G. Le Vey, L. Lanari, and A. De Luca. Rest-to-rest motion for planar multi-link flexible manipulator through backward recursion. Journal of Dynamic Systems, Measurement, and Control, 126:115-123, 2004.

C. Bermes. Entwurf einer Vorsteuerung und Folgeregelung für den Ruhelagenwechsel des polaren Doppelpendels (in German), 2005. Student Thesis (unpublished), Universität Stuttgart, Germany. 
D.P. Bertsekas. Dynamic programming and optimal control. Athena Scientific, Belmont, Massachusetts, 2nd edition, 2000.

S. Boyd and L. Vandenberghe. Convex optimization. Cambridge University Press, 2004.

R.W. Brockett and M.D. Mesarovic. The reproducibility of multivariable systems. Journal of Mathematical Analysis and Applications, 11:548-563, 1965.

D. Chen and B. Paden. Stable inversion of nonlinear non-minimum phase systems. International Journal of Control, 64:81-97, 1996.

H. Chen, A. Kremling, and F. Allgöwer. Nonlinear predictive control of a benchmark CSTR. In Proc. 3rd European Control Conference (ECC), pages 3247-3252, Rome, Italy, 1995.

S.K. Chung, C.R. Koch, and A.F. Lynch. Flatness-based feedback control of an automotive solenoid valve (accepted). IEEE Transactions on Control Systems Technology, 2006.

R.W. Chylla and D.R. Haase. Temperature control of semi-batch polymerization reactors. Computers \& Chemical Engineering, 17:257-264, 1993.

J.E. Dennis and R.B. Schnabel. Numerical methods for unconstrained optimization and nonlinear equations. Prentice-Hall, Englewood Cliffs, NJ, 1983.

S. Devasia, D. Chen, and B. Paden. Nonlinear inversion-based output tracking. IEEE Transactions on Automatic Control, 41:930-942, 1996.

D. Eberly. Derivative approximation by finite differences, 2001. Geometric Tools, Inc., Chapel Hill, North Carolina (USA), http://www.geometrictools.com.

K.G. Eltohamy and C.-Y. Kuo. Nonlinear optimal control of a triple inverted pendulum with single control input. International Journal of Control, 69:239-256, 1998.

S. Engell and K.-U. Klatt. Nonlinear control of a non-minimum-phase CSTR. In Proc. American Control Conference (ACC), pages 2941-2945, San Francisco, CA (USA), June 1993.

I. Fantoni, R. Lozano, and M.W. Spong. Energy based control of the pendubot. IEEE Transactions on Automatic Control, 45:725-729, 2000.

M. Fliess and R. Marquez. Continuous-time linear predictive control and flatness: a module-theoretic setting with examples. International Journal of Control, 73:606-623, 2000.

M. Fliess, J. Lévine, P. Martin, and P. Rouchon. Flatness and defect of nonlinear systems: introductory theory and examples. International Journal of Control, 61:1327-1361, 1995.

B. Fornberg. Generation of finite difference formulas on arbitrarily spaced grids. Mathematics of Computation, pages 699-706, 1988.

K. Furuta, H. Kajiwara, and K. Kosuge. Digital control of a double inverted pendulum on an inclined rail. International Journal of Control, 32:907-924, 1980.

K. Graichen and M. Zeitz. Swing-up of a pendubot under input constraints. In Proc. 49. Internationales Wissenschaftliches Kolloquium, pages 393-398, Technische Universität Ilmenau (Germany), Sept. 2004. 
K. Graichen and M. Zeitz. Nonlinear feedforward and feedback tracking control with input constraints solving the pendubot swing-up problem. In Preprints 16th IFAC World Congress, Prague/CZ, 2005a.

K. Graichen and M. Zeitz. Vorsteuerungsentwurf mit Eingangsbeschränkungen für nichtlineare Mehrgrößensysteme (in German). In Proc. GMA Fachausschuss 1.40 "Theoretische Verfahren der Regelungstechnik", Bosen (Germany), Sept. 2006a.

K. Graichen and M. Zeitz. Feedforward control design for nonlinear MIMO systems under input constraints (accepted). International Journal of Control, 2007.

K. Graichen and M. Zeitz. A new approach to feedforward control design under output constraints applied to the side-stepping of the triple inverted pendulum. In Preprints 4th IFAC Symposium on Mechatronic Systems, pages 181-186, Heidelberg (Germany), 2006b.

K. Graichen and M. Zeitz. Inversionsbasierter Vorsteuerungsentwurf mit Ein- und Ausgangsbeschränkungen. Automatisierungstechnik, 54:187-199, 2006c.

K. Graichen and M. Zeitz. Feedforward control design for nonlinear systems under input constraints. In T. Meurer, K. Graichen, and E.D. Gilles, editors, Control and Observer Design for Nonlinear Finite and Infinite Dimensional Systems, LNCIS 322, pages 235-252. Springer, 2005b.

K. Graichen, V. Hagenmeyer, and M. Zeitz. Van de Vusse CSTR as a benchmark problem for nonlinear feedforward control design techniques. In Proc. 6th IFAC Symposium "Nonlinear Control Systems" (NOLCOS), pages 1123-1128, Stuttgart (Germany), 2004.

K. Graichen, V. Hagenmeyer, and M. Zeitz. A new approach to inversion-based feedforward control design for nonlinear systems. Automatica, 41:2033-2041, 2005a.

K. Graichen, V. Hagenmeyer, and M. Zeitz. Adaptive feedforward control with parameter estimation for the Chylla-Haase polymerization reactor. In Preprints 44th IEEE Conf. on Decision and Control 83 European Control Conf. (CDC-ECC), pages 3049-3054, Sevilla (Spain), 2005b.

K. Graichen, M. Treuer, and M. Zeitz. Fast side-stepping of the triple inverted pendulum via constrained nonlinear feedforward control design. In Preprints 44 th IEEE Conf. on Decision and Control \& European Control Conf. (CDC-ECC), pages 1096-1101, Sevilla (Spain), 2005c.

K. Graichen, V. Hagenmeyer, and M. Zeitz. Feedforward control with online parameter estimation applied to the Chylla-Haase reactor benchmark. Journal of Process Control, 16:733-745, 2006.

K. Graichen, M. Treuer, and M. Zeitz. Swing-up of the double pendulum on a cart by feedforward and feedback control with experimental validation. Automatica, 43(1):63-71, 2007.

S. Gros, B. Srinivasan, and D. Bonvin. Robust predicitve control based on neighboring extremals. Journal of Process Control, 16:243-253, 2006.

V. Hagenmeyer and M. Nohr. Flatness-based two-degree-of-freedom control of industrial semi-batch reactors. In T. Meurer, K. Graichen, and E.D. Gilles, editors, Control and Observer Design for Nonlinear Finite and Infinite Dimensional Systems, LNCIS 322, pages 315-332. Springer, 2005.

V. Hagenmeyer and M. Zeitz. Flachheitsbasierter Entwurf von linearen und nichtlinearen Vorsteuerungen. Automatisierungstechnik, 52:3-12, 2004a. 
V. Hagenmeyer and M. Zeitz. Internal dynamics of flat nonlinear SISO systems with respect to a non-flat output. Systems \& Control Letters, 52:323-327, 2004b.

Hasomed GmbH. Product information of the double and triple pendulum. Magdeburg (Germany), www.hasomed.de.

R.M. Hirschorn. Invertibility of multivariable nonlinear systems. IEEE Transactions on Automatic Control, 24:855-865, 1979.

I.M. Horowitz. Synthesis of Feedback Systems. Academic Press, New York, 1963.

C.-I Huang and L.-C. Fu. Passivity based control of the double inverted pendulum driven by a linear induction motor. In IEEE Int. Conference on Control Applications (CCA), pages 797-802, Istanbul, Turkey, June 2003.

A. Isidori. Nonlinear Control Systems. Springer, 3rd edition, 1995.

T. Kailath. Linear Systems. Prentice-Hall, 1980.

H.B. Keller. Numerical methods for two-point boundary value problems. Blaisdell, Massachusetts, 1968.

T. Kiefer, A. Kugi, and W. Kemmetmüller. Modeling and flatness-based control of a 3dof helicopter laboratory experiment. In Proc. 6th IFAC Symposium "Nonlinear Control Systems" (NOLCOS), pages 207-212, Stuttgart (Germany), 2004.

T. Kiefer, A. Kugi, K. Graichen, and M. Zeitz. Feedforward and feedback tracking control of a 3DOF helicopter experiment under input and output constraints. In 45th IEEE Conf. on Decision and Control (CDC), San Diego (USA), 2006.

J. Kierzenka and L.F. Shampine. A BVP solver based on residual control and the MAtLAB PSE. ACM Transactions on Mathematical Software, 27:299-316, 2001.

K.-U. Klatt and S. Engell. Kontinuierlicher Rührkesselreaktor mit Neben- und Folgereaktionen. In Nichtlineare Regelung - Methoden, Werkzeuge, Anwendungen, VDI-Berichte, Nr. 1026, pages 101108. VDI-Verlag, 1993.

K.-U. Klatt, S. Engell, A. Kremling, and F. Allgöwer. Testbeispiel: Rührkesselreaktor mit Parallelund Folgereaktion. In S. Engell, editor, Entwurf Nichtlineare Regelungen, pages 425-432. Oldenbourg, 1995.

R. Köhler. Preprocessing Tool for Method-of-Lines Discretization of Process Models in the Simulation Environment DivA. Number 357 in Fortschritt-Berichte VDI Reihe 20. VDI Verlag, Düsseldorf (Germany), 2002.

A.N. Kolmogorov and S.V. Fomin. Introductory Real Analysis. Dover Publications, New York, 1975. (translated and edited by R.A. Silverman).

C. Kravaris, M. Niemiec, R. Berber, and C.B. Brosilow. Nonlinear model-based control of nonminimum-phase processes. In R. Berber and C. Kravaris, editors, Nonlinear Model Based Process Control, volume 353, pages 115-142. NATO ASI series, 1998. 
A. Kugi and T. Kiefer. Nichtlineare Trajektorienfolgeregelung für einen Laborhelikopter (in German). eÉ elektrotechnik und informationstechnik, 122:300-307, 2005.

H. Kwakernaak and R. Sivan. Linear Optimal Control Systems. Wiley-Interscience, New York, 1972.

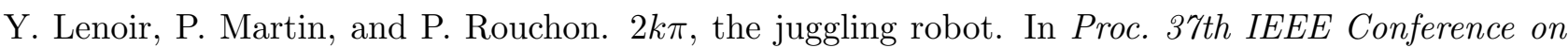
Decision and Control (CDC), pages 1995-2000, Tampa, Florida, USA, Dec. 1998.

R. Marino and P. Tomei. Nonlinear Control Design. Prentice Hall, 1995.

P. Martin, R.M. Murray, and P. Rouchon. Flat systems (mini-course). In Proc. 4th European Control Conference (ECC), pages 211-264, 1997. Also see "Flat systems, equivalence and trajectory generation" under http://cas.ensmp.fr/ rouchon/XUPS99/MMR_CDS.pdf.

G.A. Medrano-Cerda. Robust stabilization of a triple inverted pendulum-cart. International Journal of Control, 68:849-865, 1997.

T. Meurer, K. Graichen, and M. Zeitz. Motion planning and feedforward control for distributed parameter systems under input constraints. In Preprints 16th IFAC World Congress, Prague/CZ, 2005.

T. Miquel, J. Lévine, and F. Mora-Camino. Flatness-based imnproved relative guidance maneuvers for commercial aircraft. In T. Meurer, K. Graichen, and E.D. Gilles, editors, Control and Observer Design for Nonlinear Finite and Infinite Dimensional Systems, LNCIS 322, pages 271-284. Springer, 2005.

S. Mori, H. Nishihara, and K. Furuta. Control of unstable mechanical system - control of pendulum. International Journal of Control, 23:673-692, 1976.

R.M. Murray, Z. Li, and S.S. Sastry. A Mathematical Introduction to Robotic Manipulation. CRC Press, Boca Raton, Florida, 1994.

J. Neupert, A. Hildebrandt, O. Sawodny, and K. Schneider. A trajectory planning strategy for large serving robots. In Proc. SICE Annual Conference, pages 2180-2185, Okayama, Japan, 2005.

M. Niemiec and C. Kravaris. Nonlinear model-state feedback control for nonminimum-phase systems. Automatica, 39:1294-1302, 2003.

H. Nijmeijer and A. van der Schaft. Nonlinear Dynamical Control Systems. Springer, 1990.

J. Oldenburg and W. Marquardt. Flatness and higher order differential model representations in dynamic optimization. Computers \& Chemical Engineering, 26:385-400, 2002.

J. O'Reilly. Observers for Linear Systems. Academic Press, London, 1983.

H. Perez, B. Ogunnaike, and S. Devasia. Output tracking between operating points for nonlinear processes: Van de Vusse example. IEEE Transactions on Control Systems Technology, 10:611-617, 2002 .

N. Petit, M.B. Milam, and R.M. Murray. Inversion based constrained trajectory optimization. In Proc. 5th IFAC Symposium "Nonlinear Control Systems" (NOLCOS), St. Peterburg, Russia, July 2001. 
N. Petit, P. Rouchon, J.-M. Boueilh, F. Guérin, and P. Pinvidic. Control of an industrial polymerization reactor using flatness. Journal of Process Control, 12:659-665, 2002.

A. Piazzi and A. Visioli. Optimal noncausal set-point regulation of scalar systems. Automatica, 37: $121-127,2001$.

Quanser Inc. 3DOF helicopter system: product information. Ontario, Canada, www.quanser.com.

M. Rathinam and R. Murray. Configuration flatness of Lagrangian systems underactuated by one control. SIAM Journal of Control and Optimization, 36:164-179, 1998.

K. Reinschke. Lineare Regelungs- und Steuerungstheorie. Springer, 2006.

R. Rothfuss. Anwendung der flachheitsbasierten Analyse und Regelung nichtlinearer Mehrgrößensysteme (in German). Number 664 in Fortschritt-Berichte VDI Reihe 8. VDI Verlag, Düsseldorf (Germany), 1997.

R. Rothfuss, J. Rudolph, and M. Zeitz. Flatness based control of a nonlinear chemical reactor model. Automatica, 32:1433-1439, 1996.

J. Rubí, Á. Rubio, and A. Avello. Swing-up control problem for a self-erecting double pendulum. IEE Proc. Control Theory Applications, 149:169-175, 2002.

M.K. Sain and J.L. Massey. Invertibility of linear time-invariant dynamical systems. IEEE Transactions on Automatic Control, 2:141-149, 1969.

W.E. Schiesser. The Numerical Method of Lines. Academic Press, San Diego, 1991.

L.F. Shampine, J. Kierzenka, and M.W. Reichelt. Solving boundary value problems for ordinary differential equations in MATLAB with bvp4c. http://www.mathworks.com/bvp_tutorial, 2000.

L.F. Shampine, I. Gladwell, and S. Thompson. Solving ODEs with Matlab. Cambridge University Press, 2003.

L.M. Silverman. Inversion of multivariable linear systems. IEEE Transactions on Automatic Control, 14:270-276, 1969.

L.M. Silverman and H.E. Meadows. Controllability and observability of time-variable linear systems. SIAM Journal on Control, 5:64-73, 1967.

S.N. Singh. A modified algorithm for invertibility in nonlinear systems. IEEE Transactions on Automatic Control, 26:595-598, 1981.

H. Sira-Ramírez and S.K. Agrawal. Differentially Flat Systems. Marcel Dekker Inc., New York, 2004.

M.W. Spong. The swing up problem for the acrobot. IEEE Control Systems Magazine, 15:49-55, 1995.

D.G. Taylor and S. Li. Stable inversion of continuous-time nonlinear systems by finite-difference methods. IEEE Transactions on Automatic Control, 47:537-542, 2002.

C.J. Tomlin and S.S. Sastry. Bounded tracking for non-minimum phase nonlinear systems with fast zero dynamics. International Journal of Control, 68:819-847, 1997. 
M. Treuer. Entwurf von Vorsteuerungen mit Beschränkungen für Mehrfachpendel (in German), 2005. Diploma Thesis (unpublished), Universität Stuttgart, Germany. Awarded by the University's Alumni Foundation ("Preis der Freunde 2005").

V.A. Tsachouridis and G.A. Medrano-Cerda. Discrete-time $H_{\infty}$ control of a triple inverted pendulum with single control input. IEE Proceedings Control Theory and Applications, 146:567-577, 1999.

T. Utz, V. Hagenmeyer, B. Mahn, and M. Zeitz. Comparative evaluation of nonlinear model predictive and flatness-based two-degree-of-freedom tracking control design in view of industrial application, October 2006. IEEE Int. Conference on Control Applications (CCA), München (Germany), October 2006.

J.G. van de Vusse. Plug-flow type reactor versus tank reactor. Chemical Engineering Science, 19: 994-998, 1964.

A. Vande Wouwer, P. Saucez, and W.E. Schiesser. Simulation of distributed parameter systems using a MATLAB-based method of lines toolbox: chemical engineering examples. Industrial Engineering E Chemistry Research, 43:3469-3477, 2004.

J. von Löwis. Flachheitsbasierte Trajektorienfolgeregelung elektromechanischer Systeme. ShakerVerlag, Aachen (Germany), 2002.

P. Wieland. Vorsteuerungsentwurf für verteilte Systeme auf der Basis reduzierter Modelle (in German), 2005. Diploma Thesis (unpublished), Universität Stuttgart, Germany. Awarded with "Procter \& Gamble Förderpreis 2005".

P. Wieland, T. Meurer, K. Graichen, and M. Zeitz. Feedforward control design under input constraints for a tubular reactor model. In 45th IEEE Conf. on Decision and Control (CDC), San Diego (USA), 2006.

M. Wiklund, A. Kristenson, and K.J. Åström. A new strategy for swinging up an inverted pendulum. In Proc. 12th IFAC World Congress, volume 9, pages 151-154, 1993.

M. Yamakati, K. Nonaka, and K. Furuta. Swing up control of double pendulum. In Proc. American Control Conference (ACC), pages 2229-2233, San Francisco, California, June 1993.

M. Yamakati, M. Iwashiro, Y. Sugahara, and K. Furuta. Robust swing up control of double pendulum. In Proc. American Control Conference (ACC), pages 290-295, Seattle, Washington, June 1995.

R. Zanasi, C. Guarino Lo Bianco, and A. Tonielli. Nonlinear filters for the generation of smooth trajectories. Automatica, 36:439-448, 2000.

M. Zeitz, K. Graichen, and T. Meurer. Vorsteuerung mit Trajektorienplanung als Basis einer Folgeregelung (in German). In VDI-Berichte Nr. 1883, pages 795-806, GMA-Kongress, Baden-Baden (Germany), 2005.

W. Zhong and H. Röck. Energy and passivity based control of the double inverted pendulum on a cart. In IEEE Int. Conference on Control Applications (CCA), pages 896-901, Mexico City, Mexico, Sept. 2001.

Q. Zou and S. Devasia. Preview-based inversion of nonlinear nonminimum-phase systems: VTOL example. In 43rd IEEE Conf. on Decision and Control, pages 4350-4356, Atlantis, Bahamas, Dec. 2004. 